Prepared for the U.S. Department of Energy

under Contract DE-AC05-76RL01830

\title{
Masters Thesis- Criticality Alarm System Design Guide with Accompanying Alarm System Development for the Radioisotope Production L
}

\section{BA Greenfield}

December 2009

\section{Pacific Northwest}

NATIONAL LABORATORY

Proudly Operated by Battelle Since 1965 


\title{
DISCLAIMER
}

This report was prepared as an account of work sponsored by an agency of the United States Government. Neither the United States Government nor any agency thereof, nor Battelle Memorial Institute, nor any of their employees, makes any warranty, express or implied, or assumes any legal liability or responsibility for the accuracy, completeness, or usefulness of any information, apparatus, product, or process disclosed, or represents that its use would not infringe privately owned rights. Reference herein to any specific commercial product, process, or service by trade name, trademark, manufacturer, or otherwise does not necessarily constitute or imply its endorsement, recommendation, or favoring by the United States Government or any agency thereof, or Battelle Memorial Institute. The views and opinions of authors expressed herein do not necessarily state or reflect those of the United States Government or any agency thereof.

\author{
PACIFIC NORTHWEST NATIONAL LABORATORY \\ operated by \\ BATTELLE \\ for the \\ UNITED STATES DEPARTMENT OF ENERGY \\ under Contract DE-AC05-76RL01830
}

Printed in the United States of America

Available to DOE and DOE contractors from the

Office of Scientific and Technical Information,

P.O. Box 62, Oak Ridge, TN 37831-0062;

ph: (865) 576-8401

fax: $(865) 576-5728$

email: reports@adonis.osti.gov

\footnotetext{
Available to the public from the National Technical Information Service, U.S. Department of Commerce, 5285 Port Royal Rd., Springfield, VA 22161 ph: (800) 553-6847 fax: $(703) 605-6900$

email: orders@ntis.fedworld.gov

online ordering: http://www.ntis.gov/ordering.htm
}

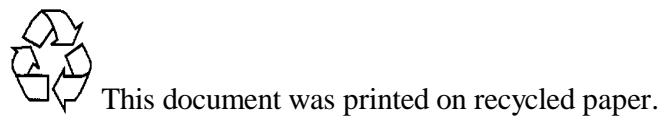


Bryec Greenficld

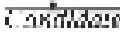

Chemical and Nuclcar Engiuccring

Triporimps

This thesis is approvext, anul it is aceptable in quality

and fism firr publisation:

Approved by the Thesis Commites:

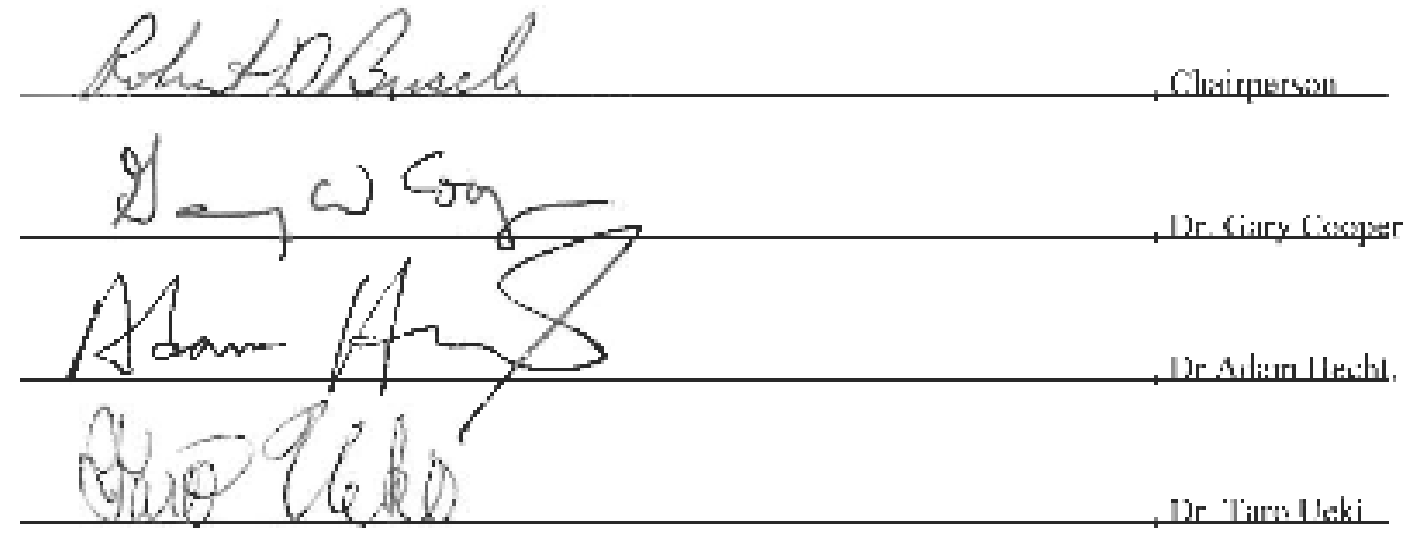


CRITICALITY ALARM SYSTEM DESIGN GUIDE WITH ACCOMPANYING ALARM SYSTEM DEVELOPMENT FOR THE RADIOCHEMICAL PROCESSING LABORATORY IN RICHLAND, WASHINGTON

\section{BY}

BRYCE GREENFIELD

ASSOCIATE OF SCIENCE - COMPUTER SCIENCE WHATCOM TECHNICAL COLLEGE 2004

\section{BACHELOR OF SCIENCE - NUCLEAR ENGINEERING UNIVERSITY OF NEW MEXICO 2007}

\section{THESIS}

Submitted in Partial Fulfillment of the

Requirements for the Degree of

Master of Science Nuclear Engineering

The University of New Mexico

Albuquerque, New Mexico

December, 2009 


\section{Acknowledgements}

I would like to thank Dr. Robert Busch. Your tireless effort and dedication is inspiring. The countless hours you have spent correcting my assignments and instructing me is truly appreciated. My work has been well received here at PNNL, and I feel that the credit is due to your patience and commitment to delivering a quality education to your students.

To all of my committee members, I would like to extend a heartfelt thank you for all that you have done. Through both undergrad and graduate levels Dr. Gary Cooper and Dr. Taro Ueki contributed so much to my education. The rigor of your classes may not have been entirely appreciated at the time, but is now more appreciated than you could know. Unfortunately I had finished my coursework before Dr. Adam Hecht arrived at UNM. However, from the very inception of this thesis writing process, he has offered his help and guidance, which was extremely valuable.

To all of those individuals at PNNL who have given me assistance over the last three years, thank you. With no exception, every staff member that I have encountered has been more than willing to lend a hand. Andrew Prichard deserves special mention. From the moment I arrived at the lab, he has painstakingly mentored me and taken the time to not just answer my questions, but to make me understand those answers. His never wavering patience and endless amounts of knowledge make him the ideal mentor.

And finally to my wife, Robin, words can not convey the heartfelt appreciation and love I have for you. Without your support I would be lost. 
CRITICALITY ALARM SYSTEM DESIGN GUIDE WITH ACCOMPANYING ALARM SYSTEM DEVELOPMENT FOR THE RADIOCHEMICAL PROCESSING LABORATORY IN RICHLAND, WASHINGTON

BY

BRYCE GREENFIELD

\author{
ABSTRACT OF THESIS \\ Submitted in Partial Fulfillment of the \\ Requirements for the Degree of \\ Master of Science \\ Nuclear Engineering \\ The University of New Mexico \\ Albuquerque, New Mexico
}

December, 2009 


\title{
CRITICALITY ALARM SYSTEM DESIGN GUIDE WITH ACCOMPANYING ALARM SYSTEM DEVELOPMENT FOR THE RADIOCHEMICAL PROCESSING LABORATORY IN RICHLAND, WASHINGTON
}

by

\author{
Bryce Greenfield
}

B.S., Nuclear Engineering, University of New Mexico, 2007

M.S., Nuclear Engineering, University of New Mexico, 2009

\begin{abstract}
A detailed instructional manual was created to guide criticality safety engineers through the process of designing a criticality alarm system (CAS) for Department of Energy (DOE) hazard class 1 and 2 facilities. Regulatory and technical requirements were both addressed. A list of design tasks and technical subtasks was compiled and analyzed to provide concise direction for how to complete the analysis.

An example of the application of the design methodology, the Criticality Alarm System developed for the Radiochemical Processing Laboratory (RPL) of Richland, Washington is also included. The analysis for RPL utilized the Monte Carlo code MCNP5 for establishing detector coverage in the facility. Based on the design methodology, significant improvements to the existing CAS were made that increase the reliability, transparency, and coverage of the system.
\end{abstract}




\section{Table of Contents}

Acknowledgements .......................................................................................................... iii

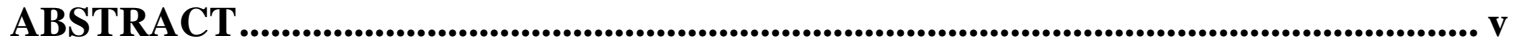

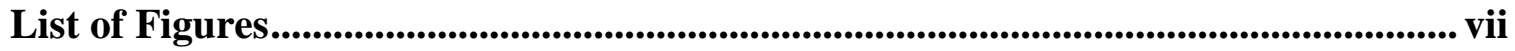

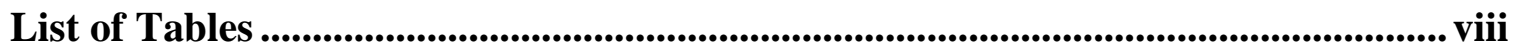

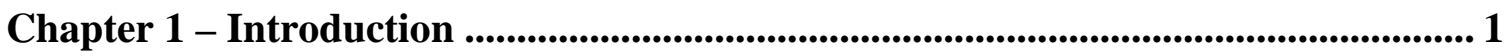

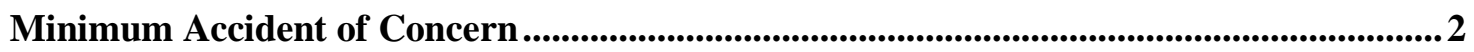

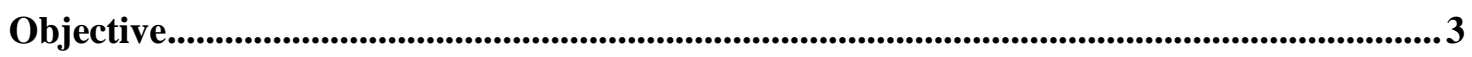

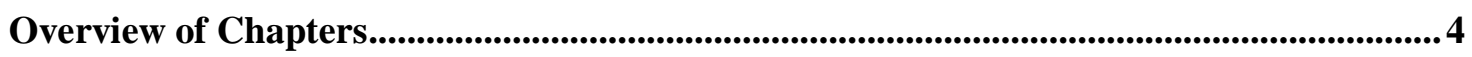

Chapter 2 - Literature Review ......................................................................... 6

Chapter 3 - Design Process Overview .......................................................................... 14

Design Methodology ............................................................................................................................... 16

Chapter 4 - Application of Guideline Methodology ............................................... 49

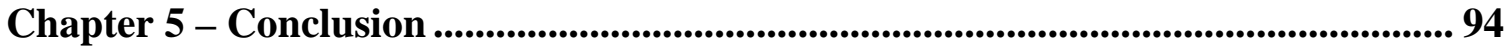

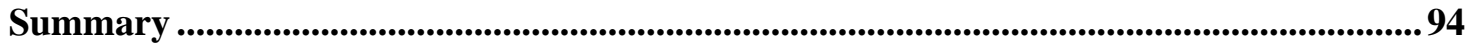

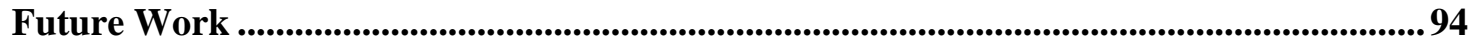

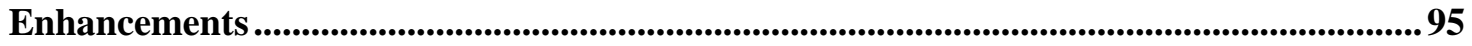

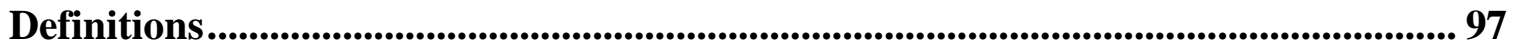

Appendix A: RPL Building Schematics with Accident and Detector Locations ...... 99

Appendix B: CAS Component Descriptions................................................................... 102

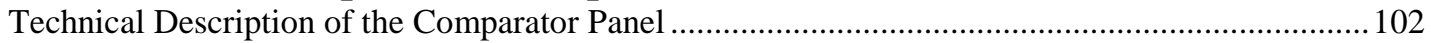

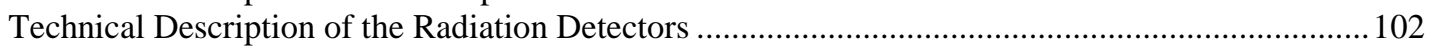

Appendix C: Material Definitions for Codes................................................................ 106

Appendix D: Schedule of Deliverables ............................................................................ 115

Appendix E: Assumptions Used in the CAS Design of RPL ................................... 116

Modeling Assumptions ............................................................................................................................ 116

Translation notes ....................................................................................................................................... 118

Appendix F: MCNP5 Input Decks ............................................................................... 119

References.............................................................................................................. 235 


\section{List of Figures}

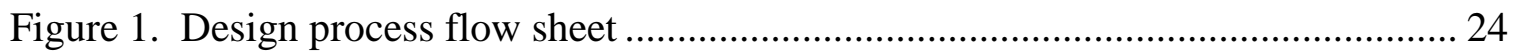

Figure 2. UNCSR dose reduction factors for various shield thicknesses ....................... 29

Figure 3. UNCSR sample slide rule for $\mathrm{U}(93.2) \mathrm{O}_{2}-\left(\mathrm{NO}_{3}\right)_{2} @ \mathrm{H} / \mathrm{X}=500$....................... 30

Figure 4. Experimental setup for the NIST Sphere Benchmarks................................... 55

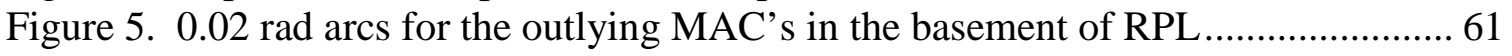

Figure 6. 0.02 rad arcs for the outlying MAC's in the first floor of RPL ....................... 62

Figure 7. RPL Basement shown with modeled accident locations and the existing

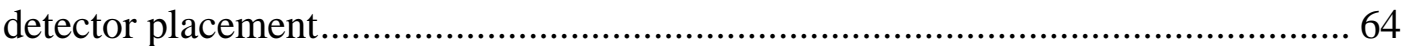

Figure 8. RPL First floor shown with modeled accident locations and the existing

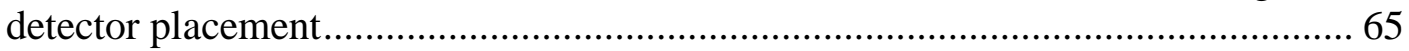

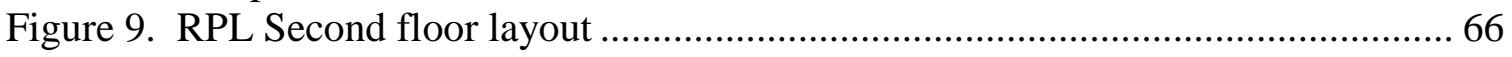

Figure 10. Standard concrete material definition of $2.3 \mathrm{~g} / \mathrm{cc}$ density............................. 70

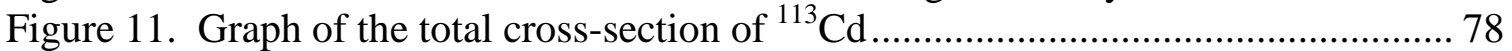

Figure 12. Neutron energy spectrum for the spontaneous fission of ${ }^{252} \mathrm{Cf}$...................... 80

Figure 13. Neutron energy spectrum grouped into $4 \mathrm{eV}$ and $20 \mathrm{MeV}$ energy bins for the spontaneous fission of ${ }^{252} \mathrm{Cf}$ plotted on a log scale ........................................... 81

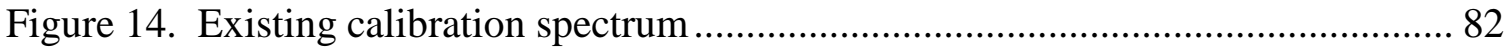

Figure 15. Average neutron energy spectrum during the Minimum Accident of Concern

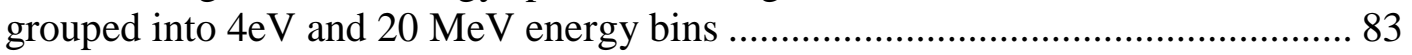

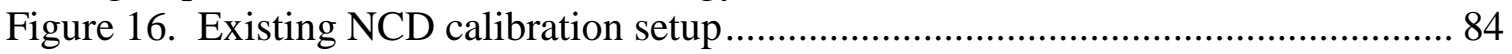

Figure 17. Revised NCD calibration setup with the added HDPE ............................... 86

Figure 18. Modified calibration spectrum grouped into $4 \mathrm{eV}$ and $20 \mathrm{MeV}$ energy bins.. 87

Figure 19. RPL Basement showing accident locations and NCD locations ................... 99

Figure 20. RPL First Floor showing accident locations and NCD locations................. 100

Figure 21. RPL Second Floor showing accident locations, no NCD are located on this

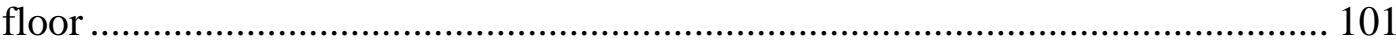




\section{List of Tables}

Table 1. Experimental normalized fission rates for ${ }^{235} \mathrm{U}$ and ${ }^{239} \mathrm{Pu}$............................ 56

Table 2. MCNP5 Computational results with comparison to experimental results......... 57

Table 3. Plutonium minimum accident scenario data .................................................. 58

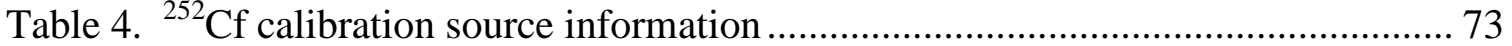

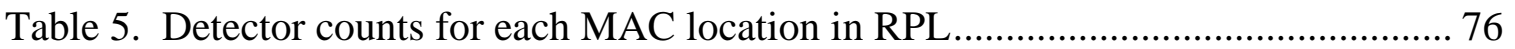

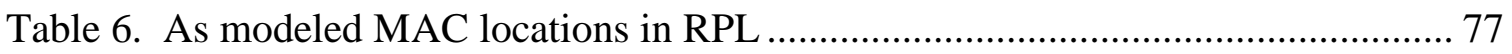

Table 7. Corresponding Data Used in the Histograms ................................................. 88

Table 8. Comparison of deterministic transport results to standard Monte Carlo results 89

Table 9. Detector counts for preliminary sensitivity analysis for maximum loading

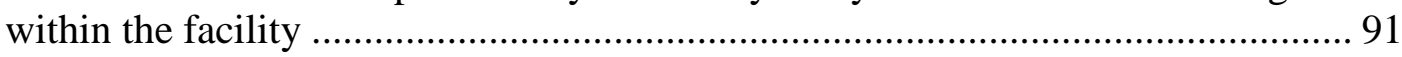




\section{Chapter 1 - Introduction}

There are a variety of hazards facing those who work with fissile material; the most severe of those hazards is a runaway chain reaction known as a criticality accident. A criticality accident can release enormous amounts of radiation that may injure or kill those who are near it. Great care is taken to minimize the chance of a criticality accident occurring, but the possibility cannot be totally eliminated. Therefore it is this minimal possibility that must be accepted and planned for.

One way to lessen the potential impact of an accident event is to install a Criticality Alarm System. The purpose of a CAS is to promptly detect a criticality accident and immediately issue an audible evacuation warning to personnel. It has been shown that rapid evacuation away from criticality accidents can considerably reduce the dose received by personnel (McLaughlin et al., 2000). Getting people out quickly can decrease injury and save lives. This is the motivation to design and implement detection systems in facilities where accidents could occur. Federal standards exist that establish the performance requirements of these systems and discuss their applicability. But nowhere, be it federal or private, is there a standard on how to design them. Currently, there exists no consolidated body of work that addresses how to design a CAS. Because these alarm systems have the potential to save lives, it is of the utmost importance that they be rigorously designed. It is the focus of this thesis to provide an instructional guide for the design of a robust CAS that will perform its functions with the highest reliability. 
All non-reactor facilities that handle greater than or equal to:

- $700 \mathrm{~g}$ of ${ }^{235} \mathrm{U}$

- $500 \mathrm{~g}$ of ${ }^{233} \mathrm{U}$

- $\quad 450 \mathrm{~g}$ of ${ }^{239} \mathrm{Pu}$

- $450 \mathrm{~g}$ of any combination of these three isotopes

are required by the Department of Energy to install and operate a criticality alarm system (U.S. DOE, 2005). For the specific performance and detector coverage requirements expected of a CAS, DOE Order 420.1B defers to ANSI/ANS-8.3-1997 Criticality Alarm Systems. ANSI/ANS 8.3.1997 is the primary standard used to judge the acceptability of a CAS design.

A CAS is generally comprised of 3 main components as described in Appendix B: a comparator panel, alarms, and radiation detectors. Selection and placement of the detectors is the most difficult part of a CAS design. The detectors must operate with a high degree of reliability and require infrequent servicing or repair. The detectors, as well as the system in general, also need to minimize the potential for false alarms. The final and most important requirement of the detectors is that they must respond immediately to the Minimum Accident of Concern (MAC).

\section{Minimum Accident of Concern}

The Minimum Accident of Concern represents the smallest accident in terms of fission yield and dose rate that the Criticality Alarm System must detect. ANSI/ANS8.3-1997 defines the MAC as one that delivers "the equivalent of an absorbed dose rate in free air of $0.2 \mathrm{~Gy} / \mathrm{min}(20 \mathrm{rad} / \mathrm{min}$ ) at 2 meters from the reacting material.” The design 
engineer must determine what likely scenarios, specific to the facility, would meet this definition. The engineer must determine the forms of the reacting materials, configuration, concentration, and quantities involved in an accident that will reach the MAC dose rates. Establishing the appropriate MAC for the targeted facility is not trivial and requires considerable effort.

The MAC represents the most difficult accident scenario that the CAS must detect. Therefore, the MAC is used extensively during the design phase to prove or disprove adequate detector coverage in the facility. ANSI/ANS 8.3.1997 establishes what adequate coverage is by requiring that no single channel may cause an alarm or fault. This means that at least two detectors will alarm for every MAC regardless of the accident location. Proving the CAS meets the coverage requirements of ANSI/ANS 8.3.1997 is the ultimate goal of the design engineer and is the benchmark for an effective Criticality Alarm System.

\section{Objective}

The purpose of this thesis is to provide a thorough guide to CAS design. Each step of the design process is discussed in detail to show why the step is needed and how to best complete it. Estimates for the time commitment of each step are included, as well as potential pitfalls that may be encountered. A complete CAS design for the Radioisotope Production Laboratory of Richland, Washington is included in this thesis to provide clear illustrations of what is being explained. The included CAS design provides corresponding examples for each topic discussed in the Design Process Overview section. 
A Criticality Alarm System designed based on this methodology will meet and exceed all current regulatory requirements.

\section{Overview of Chapters}

Chapter 2 provides a synopsis of the major federal requirements and standards that apply to CAS design. Each specific document is briefly summarized and discussed. After reading this chapter, the reader should have an understanding of the relevant regulations that drive the implementation and design requirements of a CAS. A general sense of how the regulations affect the system design is discussed. A more detailed analysis of the ANSI/ANS-8.3-1997 standard on Criticality Accident Alarm Systems completes the chapter.

Chapter 3 begins by giving a conceptual overview of what goes into the design of a CAS. A design process flow sheet is provided to illustrate how different design criteria affect the project. Following the process overview is the Design Methodology. An outline of tasks to be completed is presented as well as a technically-oriented subtask listing. The flow sheet and task outline form the foundation of how the remainder of the document is organized.

Chapter 4 uses a CAS developed for the Radioisotope Production Laboratory(RPL) in Richland, Washington to illustrate how to apply the methodology presented in Chapter 3. The task and technical subtask layout described in the previous chapter is applied to the RPL CAS. Results are given and evaluated for the MAC, coverage requirements, spectrum analysis, sensitivity analysis, and validation benchmarks. MCNP5 inputs (the facility model, validation benchmarks, spectrum 
analysis, MAC, and sensitivity analysis) are provided in Appendix D for the different RPL specific models discussed.

The conclusion portion of the thesis draws all the sections together by highlighting and summarizing the major points. The improvements made to the RPL CAS are also listed with some discussion on their potential impacts on the facility with respect to safety and operations. The potential for future work in this area is also evaluated. 


\section{Chapter 2 - Literature Review}

There are a multitude of documents that regulate the design and performance of a CAS. The final compliance memo for a CAS must not only reference the applicable regulations but include an actual copy of them in an appendix. This is done because the rules change over time. It is crucial to have a hard copy of what the rules were at the time the analysis was performed. Doing so helps avoid liability for future design conditions and performance requirement alterations as well as providing documentation for future system analyses and upgrades.

To begin evaluating the regulations the engineer needs to recognize that different facility classifications require different sets of regulations. The largest difference in regulations is whether or not the facility contains a reactor. The rules are significantly different for reactor and non-reactor facilities. This analysis focuses on CAS systems for non-reactor facilities. An important note, some of the standards discussed apply to all types of facilities; however it is just as crucial to justify why the standard is not applicable as it is to show why it is. Below is list of the documents that should be pulled and checked for relevance for non-reactor facilities.

1) DOE 420.1B Facility Safety (USDOE, 1997)

2) ANSI/ANS-8.1-1998 Nuclear Criticality Safety in Operations with Fissionable Material Outside of Reactors

3) ANSI/ANS-8.3-1997 Criticality Accident Alarm Systems

4) ANSI/ANS-8.10-1983 Criteria for Nuclear Criticality Safety Controls in Operations with Shielding and Confinement

5) ANSI/ANS-8.15-1981 Nuclear Criticality Control with Special Actinide Elements

6) ANSI/ANS-8.19-2005 Procedures for Nuclear Criticality Safety 
These are the federal regulations and standards related to Nuclear Criticality Safety and Criticality Accident Alarm Systems. There will also be company specific procedures for how to comply with these documents. If the final compliance report does not apply to both federal and company specific procedure, it will not be accepted. The language used in the final reports will vary from company to company so the format of the report itself will not be discussed. The analysis performed for a Pacific Northwest National Laboratory facility had to comply with the following internal documents:

1) MEA001 Nuclear Criticality Safety Basis Memo Evaluation, Documentation and Approval

2) NQA1 Quality Control

3) MA250 Criticality Safety Manual

4) MA500 Nuclear Material Control and Accountability Plan

5) Radiochemical Processing Laboratory Documented Safety Analysis

6) Radiochemical Processing Laboratory Technical Safety Review

7) Criticality Safety Specification 1

8) Criticality Safety Specification 2

9) Criticality Safety Specification 3

Each of the documents listed above has the possibility of impacting the design, implementation, and final compliance report. The need to thoroughly analyze each document for applicability and to make a detailed list of notes while doing so to keep track of them all is vital to the successful completion of the analysis. Because the second list of requirements is company specific, they will not be discussed in detail. Most of the company specific documents are controlled and not available for public release. Topics that are typically universal regardless of the company, like quality control and method 
validation, will be discussed to illustrate how related to these topics tasks can be conducted.

Throughout the range of ANSI/ANS documents, the same definitions are used for the terms shall, should, and may. These terms are defined throughout the standards as follows: The word "shall" is used to denote a requirement, the word "should to denote a recommendation, and the word "may" to denote permission, neither a requirement nor a recommendation. To conform with this standard, all operations shall be performed in accordance with its requirements but not necessarily with its recommendations (ANSI/ANS 8.3, 1997). To avoid misinterpreting what has been said, it is important to keep in mind how these words are defined when reading the standards.

To establish facility and program safety requirements for the DOE, a good starting point for all of these regulations and standards is the Department of Energy Order 420.1B Facility Safety. This document is the fundamental regulatory driver for a CAS in a federal facility. DOE 420.1B then breaks down what is required from a CAS, but it generally does not list the requirements. What Order 420.1B does is reference the documents that specifically list the technical requirements, which are typically ANSI/ANS standards. Order 420.1B is structured this way, no doubt, because the ANSI/ANS standards are periodically reanalyzed and updated. There are some key sections of Order 420.1B that must be read and understood:

- 4.1.1 Nuclear Safety

- $\quad 4.3$ Nuclear Criticality Safety

- $\quad$ 4.3.3 Specific Requirements 
It should be noticed that Order 420.1B augments many sections of the different ANSI/ANS standards. Why it is important to note these modifications is discussed further in Design Process Overview Task II. There is a section in Order 420.4.3.3e part 4 that addresses what is known as quasistatic criticality accidents. Quasistatic accidents are tremendously challenging to detect and are not addressed within this Thesis. Order 420.1B then directs the user to ANSI/ANS-8.1-1998, which is the overarching criticality safety standard. All other criticality related standards address implementing the general philosophy contained in ANSI/ANS-8.1-1998.

\section{1) ANSI/ANS-8.1-1998 - Nuclear Criticality Safety in Operations with Fissionable}

\section{Material Outside Reactors:}

Scope - This standard is applicable to operations with fissionable materials outside nuclear reactors, except for the assembly of these materials under controlled conditions, such as in critical experiments. Generalized basic criteria are presented and limits are specified for some single fissionable units of simple shape containing U-233, U-235, or Pu239, but not for multiunit arrays. Requirements are stated for establishing the validity and areas of applicability of any calculational method used in assessing nuclear criticality safety. This standard does not include the details of administrative controls, the design of processes or equipment, the description of instrumentation for process control, nor detailed criteria to be met in transporting fissionable materials.

This standard lays out the general methodology and techniques for how work with fissionable materials should be carried out. It thoroughly discusses what an acceptable analysis would be for meeting safety requirements, and then it discusses what must happen if this analysis is based on computer simulations. This standard also discusses how computer systems must be validated using established experimental benchmarks to ensure that the code is performing as claimed. 


\section{2) ANSI/ANS-8.3-1997 - Criticality Accident Alarm System}

Scope - This standard is applicable to all operations involving fissionable materials in which inadvertent criticality can occur and cause personnel to receive unacceptable exposure to radiation. This standard is not applicable to detection of criticality events where no excessive exposure to personnel is credible, nor to nuclear reactors or critical experiments. This standard does not include details of administrative actions or of emergency response actions that occur after alarm activation.

This standard contains a range of vital information. There is so much useful material in this standard that to discuss it all would be to almost reproduce it in its entirety. The most important parts with regard to design impact are the description of the coverage requirement, false alarm tolerance, detector failure, and the minimum accident of concern. Each of these parameters should be understood because each can impact the physical layout of the system. A brief discussion on the interplay among these parameters is useful to illustrate how one can affect another, and to show how important this standard is to CAS design.

The coverage requirement is the number of detectors that must alarm for the MAC. To meet the coverage requirement, the engineer would like the threshold (trip) setting for the detectors to be as low as possible, but if set too low, then there is a greater risk of false alarms. If the CAS is designed to meet only the minimum detector coverage requirement, then a fault at any one node takes the entire system out of compliance, thereby stopping work in the facility. However, the more detectors required for the system, then the greater the cost of installation and operation becomes. The most important piece of information this standard supplies is the general definition of the MAC.

The minimum accident of concern can be thought of conceptually as the worst case scenario for what a criticality safety engineer must design for. ANSI/ANS-8.3-1997 
defines the minimum accident of concern as "the smallest event in terms of yield and dose that a criticality alarm system must detect.” It then goes on to define the MAC even further as "to deliver the equivalent of an absorbed dose rate in free air of $0.2 \mathrm{~Gy} / \mathrm{min}$ (20 $\mathrm{rad} / \mathrm{min}$ ) at 2 meters from the reacting material.” For the system to be accepted in a DOE facility, CAS must be able to detect the MAC.

The purpose of the MAC definition is to give a clear lower limit of what must be detected, while still giving the criticality engineer some freedom with how to design the system. The standard does this by listing a minimum dose rate and by not listing how this dose rate is detected. From a practical sense not defining what the dose results from, neutrons, gammas, etc., lets the engineer decide what particles the system should detect. Having some freedom here allows the alarm system to be tailored to the individual facility.

\section{3) ANSI/ANS-8.10-1983 - Criteria for Criticality Safety Controls in Operations with Shielding and Confinement:}

Scope - This standard is applicable to operations outside of nuclear reactors with $U$-235 $U$-233, $P u-239$, and other fissile and fissionable materials in which shielding and confinement are provided for protection of personnel and the public, except the assembly of these materials under controlled conditions, such as in critical experiments. Criteria are provided that may be used for criticality control under these conditions. The standard does not include the details of administrative procedures for control (which are considered to be management prerogatives) nor details regarding the design of processes and equipment or descriptions of instrumentation for process control.

This standard outlines a possible exception to requiring a CAS in a facility that is over the mass limit cutoff but has large amounts of shielding. This obviously warrants some attention because nothing makes project managers angrier than spending thousands 
of dollars on completely unnecessary work. Without going into great detail, the standard's basis for omission of a CAS deals with sufficient shielding requirements, remote operation, and personnel restrictions.

\section{4) ANSI/ANS-8.19-2005 - Administrative Practices for Nuclear Criticality Safety:}

Scope - This standard provides criteria for the administration of a nuclear criticality safety program for outside-of-reactor operations in which there exists a potential for nuclear criticality accidents.

Responsibilities of management, supervision, and the nuclear criticality safety staff are addressed. Objectives and characteristics of operating and emergency procedures are included.

This addresses the administration of a criticality safety program. Like the other standards mentioned in this section, it references many of the other criticality focused ANSI/ANS standards including ANSI/ANS-8.3-1997 Criticality Accident Alarm Systems. This standard is more focused on how programs are implemented and documented. Because the CAS design will have to be administered adherent to this standard, it should be reviewed during the CAS design process.

\section{5) ANSI/ANS-6.1-1991 - Neutron and Gamma-ray Fluence-to-Dose Factors:}

Scope - This standard presents data recommended for computing the biologically relevant dosimetric quantity in neutron and gamma-ray radiation fields. Specifically, this standard is intended to for use by shield designers to calculate effective dose equivalent per unit fluence for neutron energies from $1 \mathrm{eV}$ to $14 \mathrm{MeV}$ and for gamma-ray energies from 0.01 to $12 \mathrm{MeV}$. Establishing maximum permissible exposure limits is outside the scope of this standard.

This is used convert gamma-ray fluence to dose in free air. The previous analysis of the RPL used this standard in conjunction with the MAC to find the smallest, and therefore most limiting, neutron fluence resultant from a criticality accident. It should be 
noted at the time of this analysis, ANSI/ANS-6.1-1991 could not be used because the standards' status had been changed from Active to Historic. The status was changed to Historic because the standard was not reevaluated within the 10 year time frame designated by ANSI/ANS. The criticality safety engineer should be cautious about using it until the status is updated.

Based on the facility description, the judgment can be made as to which ANSI/ANS standards are applicable. As stated previously, it is important to make clear arguments why or why not each standard is specifically applicable to the design. These arguments will be included in the final report and checked over by quality control engineers. Of all the ANSI/ANS standards, the most applicable is obviously 8.3-1997 Criticality Accident Alarm Systems. This document will likely guide the majority of the technical design and warrant a good deal of attention. 


\section{Chapter 3 - Design Process Overview}

Below is a brief overview of what goes into a complete CAS design. Each of the following steps is discussed in greater detail in the Methodology section. Throughout the design phase, frequent reviews of completed work and of the progress being made are advisable. In such a long process there are innumerable chances for mistakes to be made. If mistakes are made early on and go unnoticed, they can render the finished product useless. It is strongly recommended to have senior criticality safety engineers or other peers periodically review completed work. To provide an itemized summary of what lays ahead, the rather sprawling job of CAS design has been condensed into seven major tasks, which are:

I. Become familiar with the facility where the CAS will be installed;

II. Obtain, read, and summarize all of the pertinent standards and guidelines; then compare company specific requirements to others, so that the most likely scenarios for the Minimum Accident of Concern can be found;

III. Outline a schedule of deliverables;

IV. Evaluate and choose a computer simulation package to perform the analysis based on validation benchmarks that clearly demonstrate the code chosen will perform as expected;

V. Design and model the criticality alarm system for the facility;

VI. Show the calibration method follows simple and concise methodology for setting the minimum detector trip points; and

VII. Assemble the final report; 
The tasks are roughly in chronological order. Lengthy projects such as this, more often than not, require tasks to be juggled simultaneously or to be moved around to meet deadlines. This project will likely be no different. The list above was constructed to help the novice engineer move from the beginning of the project to the end with organization and traceability. 


\section{Design Methodology}

This section focuses on how to carry out the design of the CAS. Each task from the outline listed at the beginning of this Chapter is thoroughly described. The approach to each task, as well as any corresponding potential issues, is examined. Task VI contains the majority of the technical design, and it includes a numbered list of specific technical subtasks. This numbered list is then revisited in the Application of Design Methodology Chapter where the same numbering is applied to the RPL Criticality Alarm System analysis.

\section{Become familiar with the facility where the CAS will be installed}

\section{Initial Phase}

Begin by conducting walk downs of the targeted facility. Take general notes of the layout and the construction materials of the building. If a current CAS exists, make thorough notes about it; pay especially close attention to the detectors and their current locations. New CAS system installation and validation is very expensive so there is strong motivation to utilize existing components.

While in the facility, talk to people to get a sense of what day-to-day operations are like. The engineer needs to develop a sense for what typically occurs in the facility because it will help avoid incorrect assumptions during the modeling phase. Some questions to ask personnel are:

- How often are renovations performed?

- What fissile material movements occur?

- Where are operations with fissile material conducted? 
- Do people and/or laboratories move around?

- Are there any neutronically significant materials present?

II. Obtain, read, and summarize all of the pertinent standards and guidelines; then compare company specific requirements to others, so that the most likely scenarios for the Minimum Accident of Concern can be found;

After the walk downs are completed, the engineer should have a better understanding of the operations and environment that the CAS design must accommodate. Using this knowledge, pull all of the references listed in the literature review section. Do not worry initially about whether or not they are specifically applicable.

- Spend the time to read them.

- Most are not terribly long.

- Pay close attention to the scope of each document.

- Obtain hard copies of all the references because they are often required in the final CAS design report.

- Make a summary of each document including:

- Scope/applicability -specify any direct requirements for the facility.

- Technical limits.

Repeat this process for the company specific regulations. Depending on the size of the company, it may or may not have a document clerk or department. If so, these personnel can be tasked to find and assemble all the applicable documents. Also, some companies maintain libraries that will have most if not all of the documents required. For example, the Hanford Technical Library at PNNL employs fulltime librarians that can be tasked with locating project specific documentation. 
After all of the standards and guidelines have been assembled and summarized, it is time to select the most limiting accident scenarios to be modeled. It was found that inserting the summarized lists into a spreadsheet was very useful. The determination of which requirements represent the most limiting accident scenario is not always clear. For example, one standard may define a dose rate where another may define fluence. Unless the difference in magnitude for each situation is considerable, it can be difficult to discern what the most limiting parameter is. Therefore, it is recommended to perform some quick calculations to verify any conjecture.

Once an accurate assessment of the most likely minimum accident scenarios has been made, the assessment must be checked by experienced criticality safety engineers. PNNL requires that the limiting design conditions be peer reviewed, and there is a very explicit list of who can review what types of work. If for some reason, quality assurance is not required at this level, the engineer should seek it out independently. To reiterate, these limiting cases drive the analysis, so if they are incomplete, the design can be incomplete. An example of a final limiting case assessment is shown in Design Methodology Minimum Accident of Concern subtask.

\section{Outline a schedule of deliverables}

The management responsible for the project may provide a time frame or they may ask the engineer for an assessment of the expected duration of the project. It is generally beneficial for all those involved to have the person(s) with the most applicable experience construct the time line. It is much more difficult to make an accurate judgment of how long a task will take if the individual has never performed it before. 
The schedule is also highly dependent on the size of the facility that the CAS is being designed for. The length of time to complete the design phase seems to roughly scale with facility size. There are several factors that drive this relationship. The larger the facility is, the longer it will take to model. The larger the model, the longer it will take to QA. The number of potential accident locations increases with building size, which adds to both the computational time and the analysis phase. For reference, the RPL building description is given in Chapter 4 as well as schematics for each floor in Appendix A. It took approximately four months to perform the analysis and write the final report. The time it will take to draft the report depends on company requirements and individual writing speed. The final Basis Memo for PNNL was written, reviewed, and re-written in about 80 hours. More on the report is discussed later in this section.

There are a few key factors that can reduce the CAS design time. Most time saving factors are human-based. For example computer code writing skills, general criticality safety knowledge, and regulatory knowledge can all aid the design engineer in completing tasks more quickly. For all the duration times listed from this point on, it is assumed that the user has the skills of an average entry-level engineer.

\section{Evaluate and choose a computer simulation package to perform the analysis based on validation benchmarks that clearly demonstrate the code chosen will perform as expected}

ANSI/ANS-8.1-1998 requires that any code used for criticality analysis be validated prior to its use. This part of the process is really several tasks combined: choosing a code, evaluating the codes usability, and validating the codes accuracy relative to the specific situation being modeled. The goal in this step is to choose a computer simulation 
package that can show detector coverage and to perform sufficient analysis of the code package chosen, to prove that it does operate as assumed. Meeting this goal is the focus of this section, and it is highly recommended that this goal be met before the design phase is undertaken.

There are some common sense reasons for performing the tasks mentioned above, but there is also a regulation that clearly invokes the need to do them as well. DOE Order 420 mandates that all facilities categorized hazard level 1 or 2 must implement a quality assurance program to oversee and ensure the legitimacy of the work being performed. So throughout the design process all of the work performed, must be checked in accordance with the facility specific QA program. At PNNL, as it should be elsewhere, the code package used to perform $\underline{\text { ANY }}$ analysis most certainly falls into QA space.

A brief word of caution, ANY code used to perform analysis on the system that will be included in any way in the final report must be validated and put through QA. Hopefully it is obvious then that it would be wise for the engineer to pick as few codes as possible to simplify this requirement. It should also be noted that many companies maintain a list of approved code packages that are specific down to the version and release dates. If the code the engineer would like to use is not on this list, it can not be used.

Once the code has been selected, if not already familiar with it, spend a few days learning the basics. It is important to do this because the validation phase is somewhat time consuming and requires a working knowledge of the code to complete. Naturally, it is a good use of time to ensure that the work can be completed in a timely manner with the selected code package. It is just common sense to not waste time validating software 
that will never be used. After getting acquainted with how the code works, it is time to proceed with the validation phase. Note, that validation is required for every project, and must be included in the final report regardless of whether similar work has been previously performed.

Computer code validation is accomplished by modeling known, well documented, and very similar real world experiments. Then the results of the simulation are compared to actual real world experiment data. The code is deemed acceptable when it is shown to model similar situations with a prerequisite accuracy. The accuracy varies from company to company. The most important part of the validation process is the selection of relevant benchmarks.

The term, "relevant benchmark” is used in reference to established experiments that are well documented and reviewed and that clearly demonstrate the phenomena being investigated. To put it simply, if modeling fission in ${ }^{235} \mathrm{U}$, then find a ${ }^{235} \mathrm{U}$ benchmark to compare it to. But this is indeed too simple an explanation. Depending on the situation it is also usually important to match the energy spectrum, chemical state of the material, geometry, and in some cases the magnitude of the fluence. The closer the benchmark physically resembles the system being investigated, the easier it will be to argue that the results obtained from the model are indeed acceptable. Luckily there exists a collection of benchmarks that fulfill these requirements.

In the last 50 years there has been a wide variety of criticality experiments conducted all over the world. These experiments were used to gain an understanding of a range of phenomena. Since these experiments were conducted, an organization has been formed to find and assemble them into a readily accessible database. Currently that 
organization is the Nuclear Energy Agency (NEA) and their database is known as The International Handbook of Evaluated Criticality Safety Benchmark Experiments (IHECSBE) (NEA, 2008). The NEA has focused on compiling and checking the validity of the experiments and then issuing very high quality detailed reports about there findings. Many evaluated experiments also include computer input and output files for the experiment. The IHECSBE database is a widely acceptable source for validation benchmarks and offers a large variety of experiments.

\section{Design and model the criticality alarm system for the facility;}

As a certain professor of mine always used to say, "Here lies the meat and potatoes of your work." Contained in this section are guidelines on how to complete the more technical aspects of this project. Below is an outline of the design phase of the project. Each number represents a subtask of the design process.

1) Find the MAC including fluence and spectrum

2) Identify potential locations for detector placement

3) Model the facility

a. Assumptions/omissions/simplifications

b. Dimensions

c. Materials Used in Computational Models

d. Detectors

e. Source

f. Tallies

g. Accident Locations

4) Find count rates of the criticality detectors for known conditions

5) Model the Calibration/Counting Setup to find the Detector Cell Tally Efficiencies

6) Adjust the value for the detector minimum trip setting

7) Verify that adequate coverage has been obtained at the new minimum trip setting

8) Modify the Calibration Procedure 


\section{9) Apply Variance Reduction as needed \\ 10) Perform sensitivity analysis on the final CAS design}

Each numbered item is discussed in greater detail below. Chapter 4 describes these steps when applied to the design of the RPL criticality alarm system. Figure 1, a design process flow sheet, is included to illustrate how each piece of Task 5 is used to establish detector coverage. Each box colored in grey represents the output of the different subtasks. 


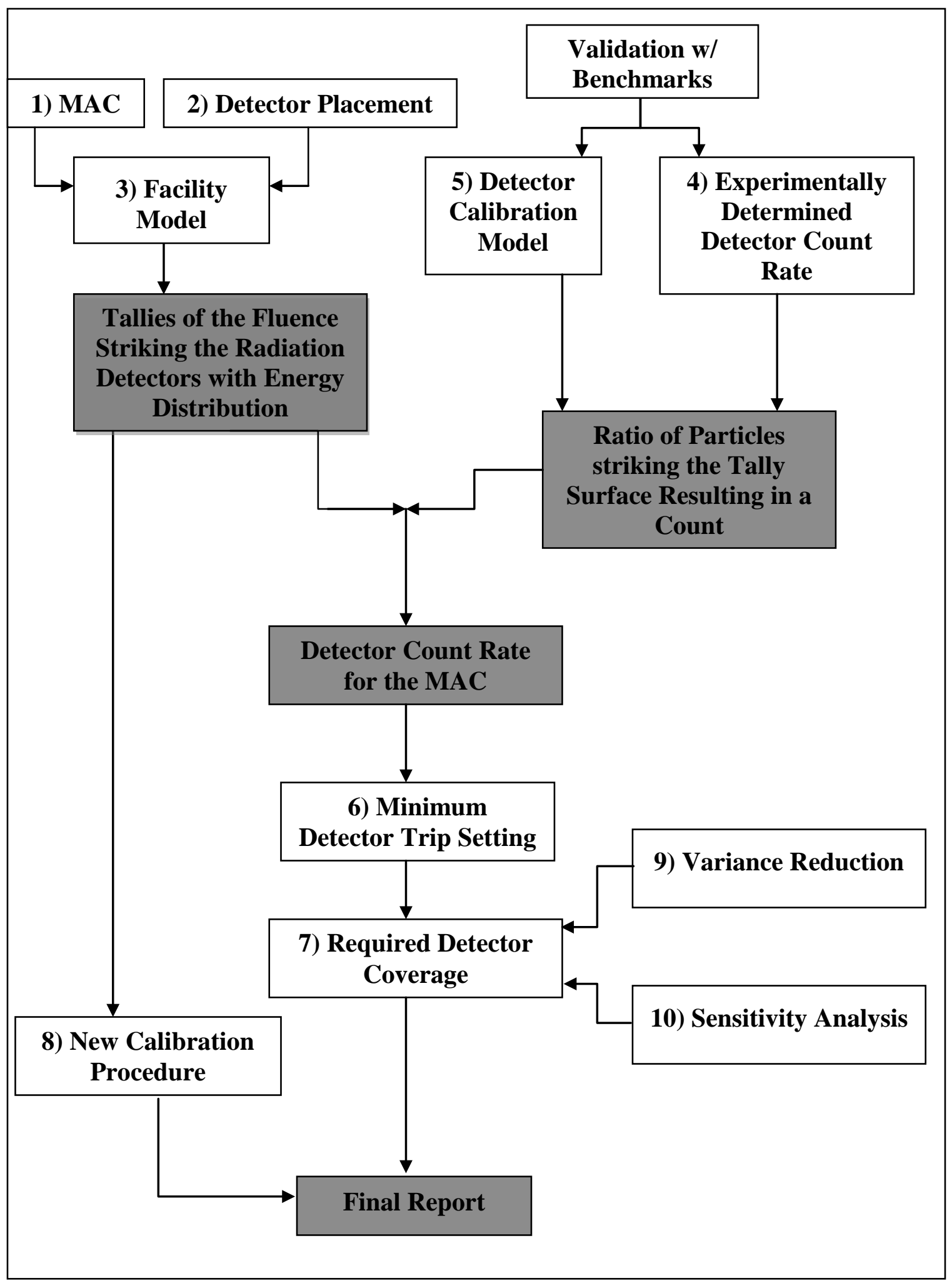

Figure 1. Design process flow sheet 


\section{Subtask 1 - Find the MAC - Fluence and spectrum}

What the engineer seeks here is the determination of which accident is the most difficult accident to detect. The justification for the need to do this was discussed previously in Design Methodology Task II. How to actually make the determination is the focus of this section.

To find the MAC, start with the definition listed in ANSI/ANS-8.3-1997. Then pick the most probable physical scenarios that are most likely to reach the ANSI/ANS8.3-1997 dose limit with the smallest number of detectable particles. So if the system employs gamma detectors, then look for the accident condition that maximizes the number of neutrons that contribute to the absorbed dose rate and vice a versa for a neutron detection system. Of course, it is not reasonable to model every conceivable accident scenario to be able to say with absolute certainty what the right answer is. What is reasonable is to pick a handful of the most likely candidates and model those.

To make a guess of what the likely accident scenarios are, it is very useful to remember the shielding properties of both gamma rays and neutrons. Low $\mathrm{Z}$ materials shield neutrons the best: increasing density, high $\mathrm{Z}$ materials shield gamma rays the best. It is clear that maximizing this discrepancy will likely generate the most limiting accident condition for the respective particles.

If both Uranium and Plutonium are present in the facility, then fission from each isotope should be looked at to determine which is more difficult to detect. Several accident conditions were analyzed for both Plutonium and Uranium including bare metal spheres, metal shielded cask type scenarios, and water reflected sources. Eventually it 
was determined for the neutron detection system in RPL that optimally moderated Plutonium and water solutions yielded the fewest neutrons for the required dose rate. Of course Plutonium and water mixtures are not very physically meaningful for chemical reasons, but that is not as important as establishing an absolute lowest bounding accident condition.

Computer models should be made for the most likely accident scenarios: one complete set using ${ }^{235} \mathrm{U}$ sources, and one complete set using ${ }^{239} \mathrm{Pu}$ sources. For the accident scenarios, the dose rate in free air from neutrons and photons must be found. Finding this dose rate per neutron and per photon is necessary to determine the corresponding minimum fluence for the accident. The smallest fluence found then identifies the most difficult accident to detect for the ANSI/ANS-8.3-1997 MAC definition.

An advantage of using MCNP5 is absorbed dose can be tallied directly by using the F6 Tally, which is discussed further in the 2) $\mathrm{f}$. Tallies subtask. MCNP5 makes a track length estimate in a designated cell for the absorbed dose there. The dose conversion equation listed as a footnote can be used to convert the result of a standard F6 tally to a final neutron fission source term. ${ }^{1}$ KENO can not directly tally absorbed dose at a location. To find dose with KENO, the fluence must first be found with the code and then converted to dose by using published fluence to dose factors. One such standard is ANSI/ANS-6.1-1991 Neutron and Gamma-ray Fluence to Dose Factors. Be aware that currently the status of this standard has been changed to "Historical," because it has not been reevaluated in the mandatory ten year review period.

\footnotetext{
${ }^{1}$ F6 Result (MeV/g)*1.00e6 (eV/MeV)*1.60218e-12 (erg/eV)*0.01 (g-rad/erg)*v(neutrons/fission)
} 
The result of the most limiting fluence found from the MAC absorbed dose analysis will be used as the source weighting factor in the facility model. This weighting factor will therefore scale the results of the simulations to the correct MAC.

\section{Subtask 2 - Identify potential locations for detector placement}

Before the facility can be modeled in subtask 3 , a preliminary number and location of the criticality detectors must be found. As stated in ANSI/ANS-8.3-1997, there must be at least two detectors that will alarm for every MAC. It is up to the user if a greater degree of coverage is desired. This initial detector placement should be viewed as a starting point, and not as the complete effort for detector placement. It should be noted that for the project, each detector represents a significant installation and maintenance cost. From a fiscal standpoint then, the engineer should optimize the design to use the fewest number of detectors to meet the coverage needs. To simply install dozens of detectors throughout the facility would be very expensive. Also, every detector must be periodically recalibrated and serviced, which adds cost over the lifetime of the system.

It was found that the fastest and simplest method for determining detector placement was to use the Updated Nuclear Criticality Slide Rule (UNCSR) (Hopper \& Broadhead, 1998). The UNCSR can be used to estimate dose resultant from a user specified fission yield. This reference allows the user to select the fission yield and then see the corresponding dose from neutrons, gamma rays, and the total at a distance. The UNCSR also provides dose reduction factors as a function of shielding thickness. This allows the yield to be adjusted for different thicknesses of interceding materials. 
Preliminary placement of the detectors needed in the facility can be made by inserting the yield from the MAC into the UNCSR. Then using engineering judgment, sufficient accident locations should be chosen for a good estimate of the coverage. For each accident location chosen, the UNCSR can be used to find the absorbed dose in rads, at a distance. It is recommended that floor by floor drawings of the facility are obtained and the appropriate scaling factors established.

To begin placing the detectors, the user must pick a threshold value of dose that represents a detectable fluence for the system. There are a number of ways to reach an estimation of the detection threshold. For both neutron and gamma ray-based systems, the average energy from fission can be used to calculate fluence as a function of absorbed dose in free air. Equation 1 shows the relationship between absorbed dose and fluence.

$$
\text { Dose in Air }=\Phi^{*} \mathrm{E}_{\text {avg }} / \rho_{\text {air }}
$$

In Equation 1, $\Phi$ is the fluence in \# of particles per MAC, $\mathrm{E}_{\text {avg }}$ is the average particle energy from fission, and $\rho_{\text {air }}$ is the density of air. Equation 1 does not contain time values or rates because the dose is assumed to be entirely from prompt fission. The values found with Equation 1 have to be corrected for the amount of intervening shielding. The UNCSR provides tables that make it easy to approximate the reduction caused by shielding. Figure 2 shows a table of dose reduction factors for prompt fission, which is included in the UNCSR. 


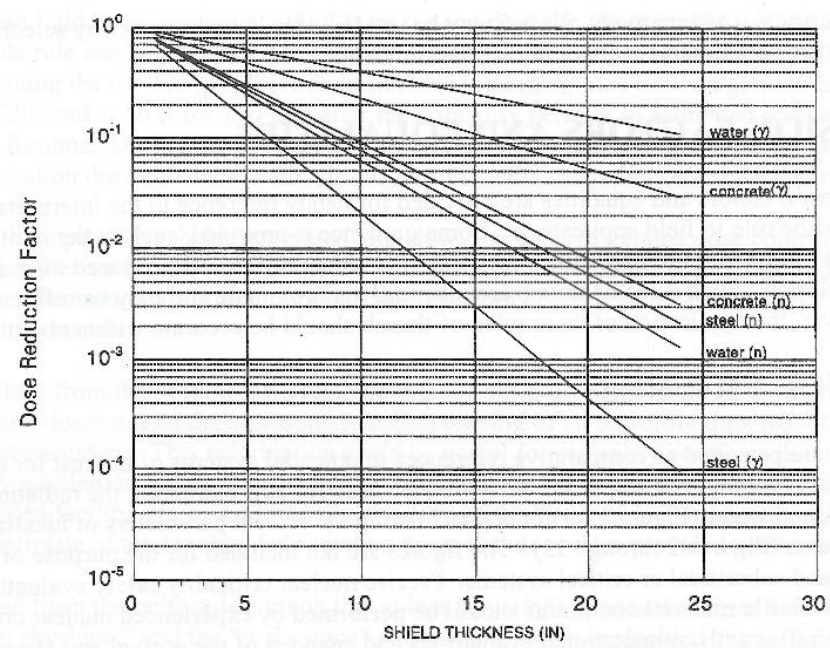

(a) prompt radiation dose-reduction factors for multiple thin shields

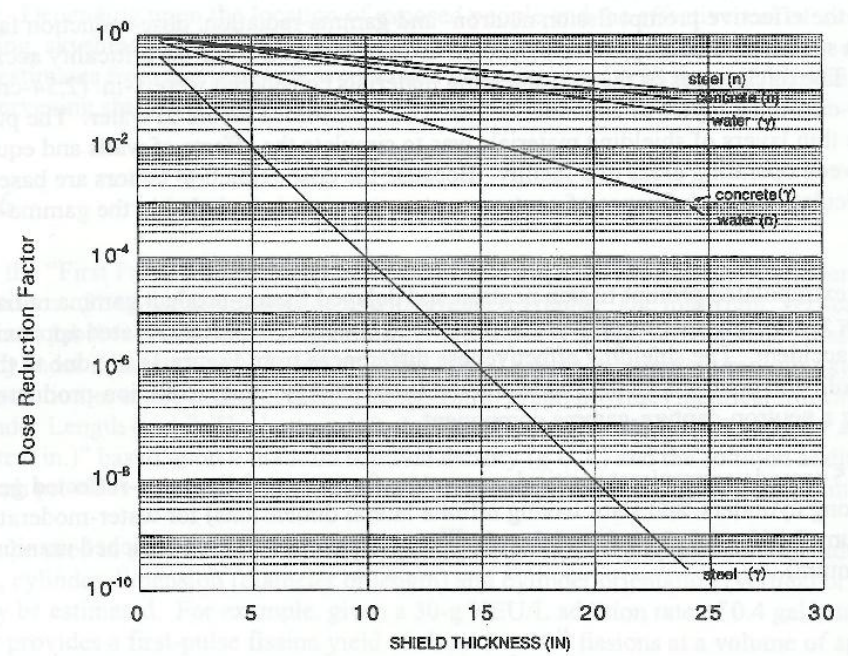

(b) prompt neutron and delayed gamma dose-reduction factors for single shields

Figure 1 Dose reduction factors for various shield thicknesses

NUREG/CR-6504,

Vol. 2

Figure 2. UNCSR dose reduction factors for various shield thicknesses

Once the dose threshold value is found and corrected for shielding, the corresponding distance for the MAC can be established with the slide rule. Figure 3 is an example of a slide rule provided in the UNCSR. 

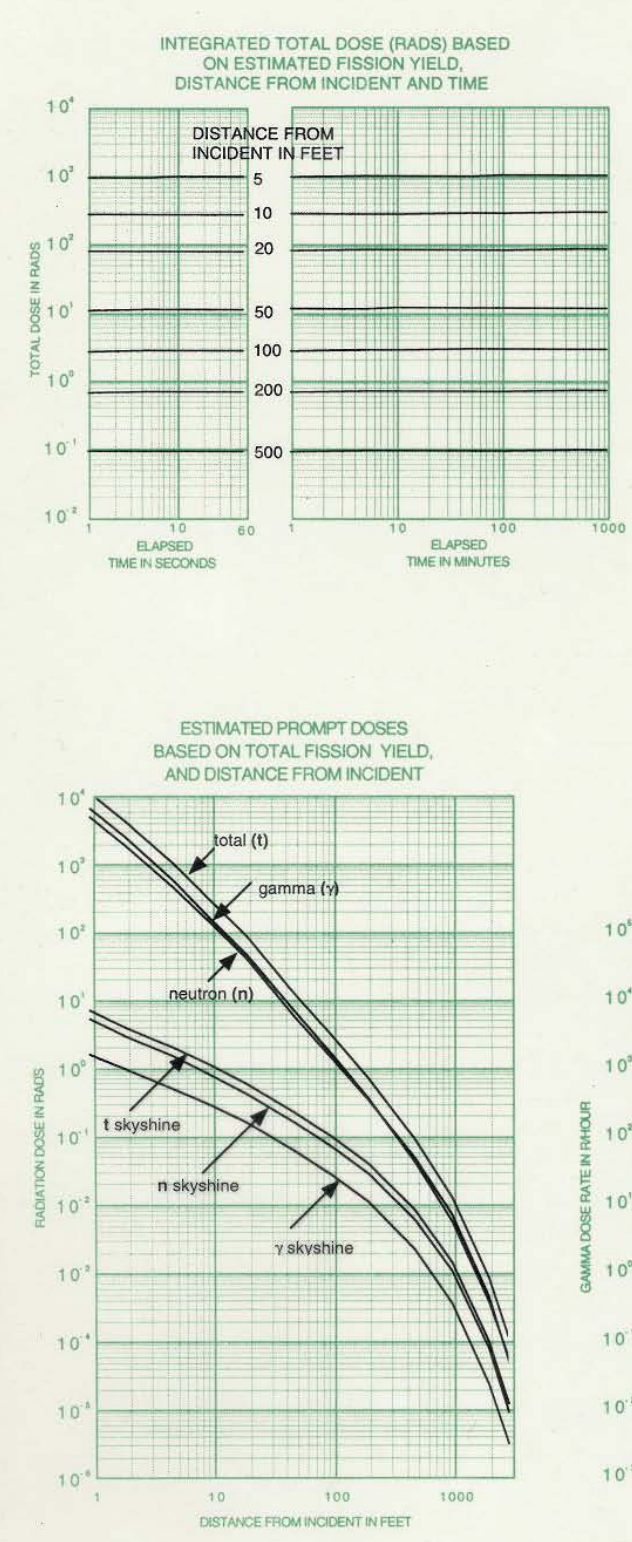

ACCUMULATED
ATE DOSE (RADS) BASED ON

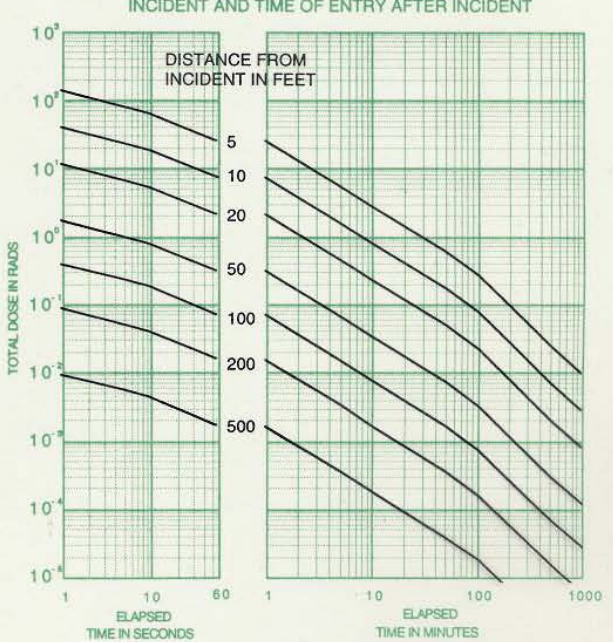

TMEINSECONOS

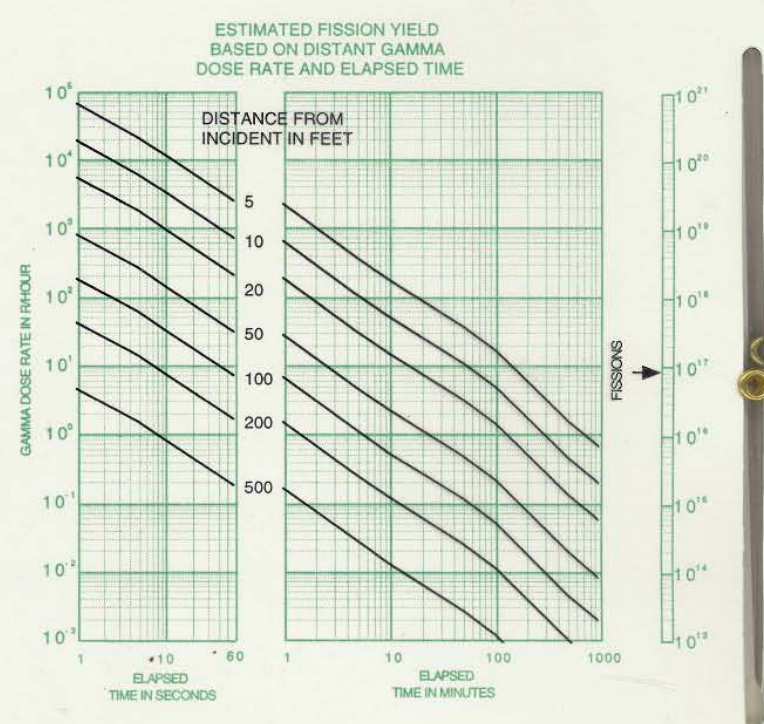

Slide 1 Solution of $\mathrm{U}(93.2) \mathrm{O}_{2}\left(\mathrm{NO}_{3}\right)_{2} @ \mathrm{H} /{ }^{235} \mathrm{U}=500$

Figure 3. UNCSR sample slide rule for $\mathrm{U}(93.2) \mathrm{O}_{2}-\left(\mathrm{NO}_{3}\right)_{2} @ \mathrm{H} / \mathrm{X}=500$ 
Using facility drawings, plot an arc of that distance value on the drawing(s) for each accident location. Areas with the most overlapping arcs will logically see the greatest number of accidents and are therefore the optimal locations to place detectors.

If the facility has multiple stories, then the process is repeated for each floor.

Once a detector layout is found for each floor, then the facility as whole can be examined. Keep in mind that particles will travel through floors and ceilings. The UNCSR can be used to find the neutron fluence reduction rates through the floors and ceilings for the MAC. Do not spend an extensive amount of time trying to get a perfect detector arrangement here because the computer simulations will provide more accurate guidance. Examples of how the arcs can be drawn are included in the corresponding subtask in Chapter 4.

\section{Subtask 3 - Modeling the facility}

\section{a. Assumptions/omissions/simplifications}

Assumptions, if any, must be clearly documented because they will need to be included as an appendix to the final report. The QA engineer will check the validity of the claims made to ensure that they all result in increased model conservatism. The guiding principle for making assumptions is that they are allowable so long as they increase the amount of conservatism, not decrease it. An example assumption could be to not model the dirt surrounding the building to save computational time. This is an example of an acceptable assumption because it only results in decreasing the number of particles that could be reflected back into the facility from the MAC. Therefore the same guiding principle still holds of being able to detect the worst case possible. An 
unacceptable assumption goes in the other direction and could then potentially invalidate the CAS. An example of an incorrect assumption is to avoid modeling the contents of offices located in the facility to save modeling time. This assumption is not conservative because taking material out of the interior of the model decreases the amount of absorption taking place. Removing a loss term inside of the facility artificially "increases" the source of the MAC, which is not conservative.

\section{b. Dimensions}

There is no substitute for good structural drawings, preferably digital CAD files. Not much needs to be said on the model geometry if it is mostly complete. Model everything that affects the particles being detected, e.g., concrete for neutrons and metals for gammas. If dimensions must be estimated, then always error on overestimating the size of bodies located within the facility and in the opposite direction for bodies located outside of it. In some instances, it may be useful to make hand drawn sketches of the facility. If this is done and the sketches are used as reference material for the model, then they must be included in the Appendices of the final report. This is a generally accepted practice.

\section{c. Materials Used in Computational Models}

Modeling the materials contained within the facility can be done with a mixture of facility inspections and published material definitions. It is very useful to physically inspect walls, ceilings, offices etc. to determine their contents. Walk-downs should be conducted to determine what amount and type of material is located in the facility. 
Generally, it will be unreasonable and unnecessary to model the interiors of every office or laboratory space. It would take too much time to include this level of detail in most facility models. But as mentioned before, the interior fill material must be included to preserve conservatism. The size of the facility, the computational resources, and the time available to perform the CAS analysis should all be considered in how the interior material will be accounted for. The sensitivity analysis conducted on the final design will show how the addition and subtraction of material in the model affects the outcome and ultimately the applicability of the design.

\section{d. Detectors}

If at all possible the detectors should be modeled to resemble the actual detectors to be installed in the facility. Geometrically accurate detector models may not be usable when the facilities get sufficiently large because computational times grow considerably. For larger facilities it can become necessary to run trillions of particles to get acceptable statistics at the detector surfaces. This number of particles can correspond to very long run times, sometimes weeks or more. Depending on project requirements and company resources, it may become necessary to look at ways of reducing the time required to run the respective codes. Methods for decreasing run times are first discussed in the tally subtask below and then again in more detail in the variance reduction subtask.

Once the computational model of the detector has been made, it must be held constant for every input. However the detectors are modeled in the facility is how they must be modeled in the calibration setup, and likewise for any other model that includes them. Keeping the modeled detectors unchanged is vital to the validity of the method 
being applied for finding the minimum trip settings and ultimately the detector coverage verification. Why keeping the detectors unchanged is so important will be discussed further in Chapter 4.

\section{e. Source}

Getting the correct source in the facility model is very important and can also be very difficult. Whatever situation that was found to be the MAC in the specific facility is the foundation of the source term for the facility model. The resultant minimum fluence found in the MAC is used as the source weighting factor in the model. MCNP5 provides a multitude of methods for modeling different sources. MCNP5 contains fission spectrums for several isotopes as well as a range of distribution functions (X5 Monte Carlo Team, 2003).

It is important to check the computer modeled source distributions for accuracy. The simulated source used in the computational model should mimic what occurs in the real world MAC. It should be understood that modeling a time dependent criticality event is extremely difficult and is beyond the needs of this project. An acceptable substitute is to make an approximate match of both the energy spectrum and fluence of the criticality accident to the simulated source. To verify that this has been done, it is recommend to use the program Vised, which is the visualization tool provided by RSICC with MCNP5. Vised has proven invaluable for catching the numerous source distribution errors made along the way. It is highly recommended to spend some time becoming familiar with the array of uses Vised has to offer. More specifically, time should be spent learning how to check source distributions using the particle tracking features. Vised will 
"run" a user designated number of particles through the model using what has been defined as the source. Vised will then produce a picture that will show a variety of useful information about what occurred with the source particles, making it very easy to see errors.

\section{f. Tallies}

The tallies chosen for the model will yield the results that are used to determine whether or not a detector has alarmed. The tallies can also be used in clever ways to decrease computational run time. MCNP5 provides eight basic tallies for recording particle information but for this project only four of them are of any use (X5 Monte Carlo Team, 2003). The tallies of interest for this design are the F1, F2, F4, and F6. Correspondingly they are surface current, surface flux, track length estimate of cell flux, and the track length estimate of energy deposition. Equations 2, 3, and 4 show, respectively, the F1, F2, and F4 tally equations. The F6 tally equation is merely the F4 cell flux tally multiplied by a heating function.

$$
\begin{aligned}
& \mathrm{F} 1=\int_{E_{i}} d E \int_{t_{j}} d t \int_{\Omega_{k}} d \hat{\Omega} \int d A|\hat{\Omega} \cdot \hat{\mathrm{n}}| v n(\overrightarrow{\mathrm{r}}, \hat{\Omega}, E, t) \\
& \mathrm{F} 2=\frac{1}{A} \int_{E_{i}} d E \int_{t_{j}} d t \int d A \phi(\overrightarrow{\mathrm{r}}, E, t) \\
& \mathrm{F} 4=\frac{1}{V} \int_{E_{i}} d E \int_{t_{j}} d t \int d V \phi(\overrightarrow{\mathrm{r}}, E, t)
\end{aligned}
$$


The units are important to note for the output of each tally. In their standard modes, the F1 tally has units of number of particles, the F2 and F4 tallies both have units of particles/cm^2, while the F6 tally has units of MeV/gram. MCNP5 will attempt to calculate the corresponding areas, volumes, or masses for each tally but is prone to error (X5 Monte Carlo Team, 2003). It is recommended that the user supply any and all of the aforementioned parameters by using the tally modification cards available in MCNP5.

The F4 tally was found to be the most reliable for tallying fluence. It should be noted that the method MCNP5 uses to calculate the F2 surface flux contains a large angle cosine approximation, which was found for this investigation, to report larger fluence values than did the F4 tally. The surface flux approximation used in the F2 tally for large angle approximation is given in Equation 5 (X5 Monte Carlo Team, 2003). The parameters in Equation 5 are $\mathrm{W}$ for the particle weight, $\delta$ for surface thickness, $\mathrm{A}$ as the surface area, $\mathrm{n}$ for the unit normal vector, and $\Omega$ as the particle position vector. Therefore, to be conservative, the decision was made to only use the F4 tally for the detectors in the building and calibration models.

$$
\bar{\phi}_{S}=\lim _{\delta \rightarrow 0} \frac{W \delta}{A \delta|\hat{\Omega} \cdot \hat{\mathrm{n}}|}
$$

The F6 tally worked very well for the MAC determination because it was very easy to show the conversion from $\mathrm{MeV} /$ gram to the absorbed dose value listed in ANSI/ANS-8.3-1997. The F1 current tally can be used to find amounts of reflection provided by different pieces of the facility. This information can then be used to greatly speed up the computational time by creating ALBEDO boundary conditions or modified 
cell importance. Run times may be decreased by up to 40 percent using these techniques. This significant decrease in run time is mostly attributed to limiting the amount of time spent on tracking particles in the sand surrounding the facility. Another way to decrease the time spent tracking particles in sand is to change the composition from pure Silicon Dioxide to include a small fraction of Boron. So many collisions can occur in the surrounding sand that a small amount of Boron makes large contributions to absorption. The discussion on tallies and variance reduction more specific to the needs of this project is continued in the Variance Reduction subtask below.

\section{Subtask 4 - Find count rates of the criticality detectors for known conditions}

A bridge must be made between what is tallied in the model to what is counted in reality. The first step in establishing this link is to find a count rate for the detectors in a known configuration that can be modeled in subtask 4 with the same code packaged used for the analysis. The quantity sought in this task will be used in conjunction with the Detector Cell Tally Efficiency to ultimately determine what count rate the minimum trip setting will be.

One possible source of well-documented count rate data would be any existing calibration procedures or operations for the detectors in question. If the calibration procedure has already been developed, then there will be extensive records for count rates as a function of source activity. If this is the case, then acquire all the data regarding the calibration setup that would be needed for modeling it in the designated code package and proceed to subtask 5. If the calibration procedure does not exist then more discussion is needed. 
The bulk of how the calibration procedure is developed and implemented is discussed in subtask 8 and hence out of the scope of this subtask. But to proceed with next task, modeling the calibration setup to find the DCTE, some preliminary work for the calibration must be conducted here. For reasons that will be discussed in subtasks 6 and 8 , it is important to establish a set of 3 or 4 core detectors that will be used for finding the count rates in this subtask. This set of core detectors will be important for establishing the minimum trip setting and instating the calibration method in subtasks 6 and 8, respectively. Ensure that these core detectors are easily traceable and identifiable. Once this set of detectors has been established, find at least one set of count rates for each in a well defined counting setup. The better the counting setup is documented, the easier subtask 5 becomes.

\section{Subtask 5 - Modeling the Calibration/Counting Setup to find the Detector Cell Tally Efficiencies}

The next piece of the puzzle is to find the detector cell tally efficiency (DCTE), which is basically the computational equivalent of the detector efficiency. The DCTE has the units of real world detector count rate divided by the number of simulated particles entering the modeled detector. The quantity being sought here is the fluence striking the modeled version of the detector in the same geometry that the experimental count rate was obtained in. The following provides a simple example of how the DCTE is applied.

Hypothetically, during the MAC runs in the facility, a detector recorded 1000 particles striking it. Using an exact replica of the facility detector in a model of a known counting setup, for a source of weight $\mathrm{W}, 750$ strikes were recorded. The actual count 
rate data obtained in the same setup as was modeled, with the same geometry and source used as weight W, was found to be 500 counts per second. Dividing the recorded count rate of 500 counts per second by 750 simulated strikes recorded yields a DCTE of 0.66. The DCTE can then be applied to the number of particles recorded in the MAC scenario to find an actual count rate. Multiplying the simulated 1000 particle strikes found in the facility model by the DCTE of 0.66 , equates to a real world count rate of 666 counts for that detector for that MAC. This argument is valid because ANSI/ANS-8.3-1997 states that the MAC is assumed to happen in 1 millisecond, or essentially instantly.

The reason for the assumed duration of the MAC in ANSI/ANS-8.3-1997 is because modeling time dependent criticality is very challenging. Currently there is no widely accepted simulation package that will accurate depict a time dependent criticality event. The current solution for modeling a criticality accident is to simply eliminate the time dependence and assume a prompt yield, thereby ignoring the delayed contributions to the total yield. Experience with criticality accidents has shown that this prompt burst assumption is valid for all non-delayed critical excursions (McLaughlin et al., 2000). Order 420.1B does not require CAS be able to detect delayed critical accidents. Therefore, the source can be weighted by the number of particles resultant from the MAC and not the time dependent fluence.

The DCTE also provides a way to check the validity of the experimentally observed count rate and efficiency. The DCTE allows the computer simulated fluence at the detector location to be compared to the fluence recorded in the real world experiment. If the computational fluence striking the detector versus the recorded count rate is considerably different than the experimentally determined detector efficiency, then 
something may be amiss. If necessary a simple solid angle approximation should identify which is wrong -the model or the experiment.

Because the DCTE is so important, the setup used to calibrate the nuclear criticality detectors needs to be modeled with greater precision than the building model. There will be activity data for whatever source is used to find the count rates or to calibrate the detectors. It is important for some, but not all sources, to very accurately

decay correct their activities. For example, the neutron source ${ }^{252} \mathrm{Cf}$, has a half life of only 2.6 years so it is very important to correct for the change in activity over time. Make sure to use the same energy bin structure for the calibration model as was used for the tallies in the facility model. The same goes for the orientation and configuration of the detectors in each model. Finally, it is very important that the exact same tally be used for both the calibration and the facility models.

\section{Subtask 6 - Adjust the value for the detector minimum trip setting}

In theory the minimum trip setting is desired to be as high as possible to avoid false alarms. In practice the engineer wants this value to be as low as possible to make the system design easier. It is advised to take a preliminary look at the count rates being recorded for the detectors in the facility model and the coverage requirements to make an initial trip setting approximation. Based on the radioactive material inventory in the specific facility, an assessment of the likelihood of a false alarm resulting from internal radioactive sources should be made.

With regard to false alarms, it is far more important to get the minimum trip setting as high as possible for gamma detection systems than it is for neutron detection 
systems. This is because there are far more natural sources of gamma radiation that could potentially cause a false criticality alarm than there are for neutrons. However, with regard to minimum accident detection it is more important to get the trip setting as low as possible for the neutron based systems than it is for the gamma. Neutron-based systems are not really affected by cosmic radiation sources like their gamma counterparts but neutrons levels are typically lower during a MAC making them more difficult to detect. There is no hard and fast way to identify the minimum trip setting. Only a best guess with sufficient documentation can get a reasonable answer.

\section{Subtask 7 - Verify that adequate coverage has been obtained at the new minimum trip setting}

The final steps in the design are focused on proving and optimizing the detector coverage of the facility. Enough data has been determined to evaluate the detector coverage in the facility. General intuition about detector and accident placement has already been discussed, so proceed with proving that the system detects the MAC everywhere in the facility. Once the coverage has been verified at the minimum trip setting by using the DCTE, then the analysis in regards to detector placement is completed. After the detector coverage has been shown, focus should be shifted to the spectrum incident on the detectors. All that remains is to modify the calibration procedure to better mimic the average spectrum observed at the detectors in the facility model during the MAC. 


\section{Subtask 8 - Modify the Calibration Procedure}

When the coverage has been thoroughly proven, it is time to take the spectrum incident on the tally surfaces of the facility and compare it to the calibration models' incident tally spectrum. It makes sense conceptually to calibrate the nuclear criticality detector to a similar spectrum of what it is expected to detect. There are a variety of ways the calibration source spectrum can be modified. The methods are somewhat different for neutrons and gammas.

For neutrons, low $\mathrm{Z}$ materials can be used to increase the ratio of thermal to fast neutrons, or conversely cadmium can be used to almost entirely eliminate neutrons below about $0.4 \mathrm{eV}$. Reflection can be increased by placing materials behind the detectors, which increases the fluence hitting the detectors. Increasing the fluence hitting the detector may be crucial if significant portions of the fluence are lost when the spectrum is modified to better emulate what is seen in the facility.

Gammas require slightly different treatment than neutrons. Equation 6 gives the formula for the linear photon attenuation coefficient $\mu$.

$$
\mu=\tau+\sigma+\kappa
$$

Equation 6 contains the three main physical processes that remove or slow photons in a beam, which are $\tau$ for the photoelectric effect, $\sigma$ for the Compton Effect, and $\kappa$ for pair production. Using these three parameters, materials can be found to modify the spectrum in a targeted way. Contributions from Rayleigh scattering and photonuclear effects can usually be ignored because they are small in comparison to the three processes in Equation 6. Gamma sources are generally cheaper and have a larger intensity than 
neutron sources. So eliminating too much of the incident beam is not as significant a concern as it is for neutron sources.

\section{Subtask 9 - Variance Reduction}

It should be readily apparent that even in small facilities the volume of the radiation detector is many orders of magnitude smaller than that of the facility. Computationally this presents a problem that is increasingly exacerbated as facility size grows. In stochastic codes, the statistics get ever worse as the detector to building volume ratio decreases. Getting usable statistics can become very arduous for large models. The run times necessary to produce tolerable variance can become unrealistic. The ensuing discussion addresses techniques for reducing computational run times by providing a few possible solutions.

It is extremely important to note that incorrectly applying variance reduction techniques can completely invalidate the results and worse still the errors can be very hard to catch. Each code package has a variety of options available for reducing variance in a problem. There are code specific options, such as invoking deterministic transport for Monte Carlo codes, and there are techniques based on engineering intuition like omitting the material surrounding the facility to decrease time tracking particles there. The following is a brief list of other code specific variance reduction methods:

- Minimum Energy Cutoffs

- Importance/weight Cutoffs

- Weight windows

- Simplified Scattering treatments

- Source direction biasing 
Each technique must be thoroughly analyzed before it is implemented. It should be noted that many organizations' quality control programs explicitly disallow some or all code-invoked variance reduction techniques. Next is a list of engineering-based simplifications that can significantly decrease run times:

- Reduction of MAC locations in the facility needed to show full coverage

- Modifying the numbers of particles being run according to ease of detection in a location

- Form arguments based on proving worst case scenarios to eliminate situations that are obviously easier, simpler, etc.

Each type of simplification has drawbacks and should be used with caution. If any method, be it code specific or engineering based, is used to reduce variance of computations, it must be well documented and peer reviewed.

\section{Subtask 10 - Perform sensitivity analysis on the final CAS design}

Sensitivity analysis is the investigation of how different factors could affect the operations of the alarm system. For example, in the computer model, fill all of the rooms within the building with "paper" to see how the neutronics are affected. This is done to say that even in the almost totally inconceivable event of the building turning into a paper repository, the alarm system will still function properly. It might sound outlandish that this type of analysis would need to be performed, but knowing how tolerant the alarm system is to potential facility modification can add credibility to the design.

Sufficient sensitivity analysis should be conducted so that clear bounding limits can be included in the final design memo for what can, and cannot be done, to/in the facility and not interfere with the CAS. The data collected during facility inspections is 
especially useful here. Much of what is investigated here will be based on what typically occurs in the facility. Depending on the facility, the following parameters could be important to investigate:

- Modifications and remodeling

- Movement of large amounts of various materials into and out of the facility

- The effect of fire suppression systems

- Changes to the material surrounding the facility

This analysis is done just as much to say what the CAS will not tolerate as it is to say what it will. Both are important for establishing the bounding operational tolerances that the system can endure.

\section{Show the calibration method follows simple and concise methodology for setting the minimum detector trip points}

After all the technical analysis is completed and reviewed, it is time to make the recommendations of how to perform detector calibration and movement. It is crucial to make recommendations and corresponding instructions as clear and concise as possible. First, it is a good engineering practice to not over complicate things. A saying from another professor applies in this instance, "keep it simple, stupid.” The more instructions there are, the greater the chance of error. Those who have to implement the recommendations should receive instructions that give abundant measurements to a single common reference point. They should see very few, if any, ambiguous words like around, near, or approximately. All dimensions should be given in one system: mks, cgs, etc. 
The second reason to keep things clear and concise is for the regulators. One of the drivers for redoing the analysis of RPL was that the DOE found the minimum trip setting for the neutron detectors to be very poorly worded. What the DOE wanted was a clear minimum trip setting that was easy to confirm or refute. The old criterion was related in terms of absorbed dose per source particle referenced to a Plutonium-Beryllium source. The new definition is, "The neutron detectors in RPL are set to alarm at 600 counts per minute, which corresponds to an absorbed dose rate in free air of 80mrem/hr." -a clear improvement.

\section{Assemble the final report}

The structure of the final report is likely to vary greatly from institution to institution, so it is of no use to go into great detail about how to write it. Also the final report drafted for PNNL is classified as business sensitive, thereby prohibiting it from being used outside of the organization. Accordingly the time needed for writing the report is likely to fluctuate considerably. It took approximately 80 hours to draft, review, rewrite, review again, and finalize the report as a whole. For time estimating purposes it should be taken into consideration that the PNNL report requirements did not seem excessive. It should also be noted that the 80 hour time estimate was not all used at once, but rather over the duration of the project. The report drafted for PNNL contained several sections that were not affected by technical design changes so they could be written ahead of time. Writing report sections early on is highly recommended. The sooner the sections are written, the sooner they can be reviewed. Having fewer sections to go over in the final review will greatly expedite the final submission process. To give 
a general sense of what may be included in the final design report, an outline is provided below.

1) Introduction-State the purpose and scope of the evaluation

1.1) Review of Previous Methods

2) Description-Describe the process/system

3) Requirements Documentation

Indicate specific DOE Orders and Guides, ANS Standards, Code of Federal Regulations or other requirement documents that are uniquely applicable to the analysis

4) Methodology

\section{1) Evaluation Methodology}

When using computer codes, indicate what codes and cross section libraries were used and reference the applicable code configuration control information and the code documentation. Identify the type of computing platform.

4.2) Benchmark Evaluation and Bias Determination

List the benchmark experiments chosen and discuss their applicability to the problem. Show the calculation of the bias and the uncertainty in the bias. Describe how the bias is applied to the results of this evaluation.

5) Evaluation and Results

Provide a detailed description of any models used. Reference code generated drawings in an Appendix. State assumptions and simplifications made. Include all calculation results.

7) Design Features|changeslimprovements

8) Summary and Conclusions

State the systems, processes or facilities that the analysis is applicable to and indicate any limitations on the evaluation.

9) References 
List all original references. Avoid referencing documents that reference other documents.

Appendix Containing Materials and Compositions

List and reference all materials. Include atom densities.

Appendix Containing Sketches and Drawings

Include sketches of the actual process (hand-drawn sketches are generally acceptable and expected) and as-built or design drawings.

Appendix Containing Code Input Listings

Include input listings for all computer code runs.

One final note on the report - a hard copy of all the references used along the way was required to be inserted as an Appendix. The definition of references at PNNL was not as expected, so it would be wise to get a clear description of what a reference is before even conducting the building walk downs. 


\section{Chapter 4 - Application of Guideline Methodology}

This set of tasks deals with the technical application of the CAS design methodology. The CAS designed for RPL is used as an example of the design methodology. Sample MCNP5 computer code inputs are listed in Appendix F for the RPL facility model, the detector calibration setup, the MAC evaluation, and the validation benchmarks. These inputs will help to guide the user through the modeling phase and ultimately the detector placement.

\section{Familiarization with the facility where the CAS will be installed;}

Over the 6 month duration of this project, roughly a dozen facility inspections were conducted. These inspections provided a solid fundamental understanding of the facility and the operations conducted therein. To form a better understanding of how the system works, a test of the existing criticality alarm system in the building was observed. This is highly recommended because seeing the test put into perspective how the testing procedures were performed.

Until the test of the CAS was witnessed, it had not been readily apparent that the people carrying it out did not have very technical backgrounds. This observation is important because it brings up a very crucial point; the procedures must be written for those who have to read them. Rigorous technical analysis can be performed but if the conclusions drawn from it can not be communicated clearly to those who must enable them, then the analysis itself is trivialized. 
The inspections of the facility repeatedly proved to be useful so it is highly recommended to conduct them frequently. Doing so can make many facets of the project go more efficiently.

\section{Obtain, read, and summarize all of the pertinent standards and guidelines; then compare company specific requirements to others, so that the most likely scenarios for the Minimum Accident of Concern can be found;}

After much debate three scenarios, with two different sources, were assumed to be the most likely limiting cases that would reach the MAC. The three cases contained either pure ${ }^{235} \mathrm{U}$ or pure ${ }^{239} \mathrm{Pu}$ in an optimally moderated water filled sphere as the source. The three cases were: a bare sphere, a water reflected sphere, and an iron reflected sphere. These three cases were chosen because they resemble some possible physically relevant accident scenarios in the facility. The bare and water reflected spheres were chosen to represent laboratory type accident scenarios. RPL is a radioisotope production facility where much bench top radiochemistry takes place. The iron reflected sphere was selected because it resembled an accident occurring in a shipping cask. A significant amount of material is moved in and out of the facility, including into and out of the High Level Radiochemistry Hot cells. Then all six permutations of these scenarios were modeled to find the most limiting fluence during the MAC. The result of the limiting plutonium accident runs with the final determination of the most limiting scenario is presented in this chapter under Subtask 1. 


\section{Outline a schedule of deliverables;}

Assembling the schedule of deliverables was taken care of by the project manager

and is included in Appendix D. The project manager assembled a preliminary timeline, and then asked for feedback on whether the timeline was acceptable. It is very important that the timeline is carefully examined because it is the criticality safety engineer that is responsible for the majority of the work. Voice any concerns as soon as they are uncovered because it gives management more time to address any issues, which is in everyone's best interest for completing the project. The single largest use of time for this project was the development and quality analysis of the facility model. It should be noted that no results are acceptable for the facility model until after it has been put thru QA. This makes it a high priority to finish as soon as possible. Do not put off either job.

\section{Evaluate and choose a computer simulation package to perform the analysis based on validation benchmarks that clearly demonstrate the code chosen will perform as expected;}

The decision was made early on to use MCNP5 because it was felt that it had the capacity to perform the analysis, and it had been accepted by the Quality Assurance program at PNNL. To ensure that MCNP5 could perform as assumed, a series of benchmarks were modeled to prove that it was indeed an appropriate tool for the design. This task goes through the process of validation per the quality control requirements of PNNL.

MCNP5 was used extensively for modeling many aspects of this project. An MCNP5 model was created for each of the following;

- A thorough representation of RPL 
- 6 different accident scenarios involving ${ }^{235} \mathrm{U}$

- 6 different accident scenarios involving ${ }^{239} \mathrm{Pu}$

- The current setup used for calibrating the $\mathrm{BF}_{3}$ Nuclear Criticality Detector(NCD) to a specified dose rate

- The recommended modified calibration setup

- 6 different scenarios for sensitivity analysis

It is necessary then to model a set of accepted, experimentally confirmed, benchmarks that resemble the models created above with the same code package. Modeling these benchmarks in the same code allows experimentally confirmed results to be compared to those received from simulation. Showing that the simulation correctly reproduces the published physically similar benchmark results therefore validates the use of this code for the work being performed.

The benchmarks selected to validate the models described above were all taken from the Nuclear Energy Agency International Handbook of Evaluated Criticality Safety Benchmark Experiments, September 2008 edition (IHECSBE). The benchmarks in the IHECSBE are an extensive set of criticality experiments that have been thoroughly peer reviewed and modeled in a variety of computer codes. The physical details of each experiment, as well as their inherent uncertainty, are listed for each benchmark. Experimental results are given with how they were obtained, detectors/type, location, etc. Then these experiments are reproduced using a common software simulation package. Results from the simulations are included in the write up of the benchmarks and generally an accompanying input file is also listed. Such an extensive degree of detail in these benchmarks allows the user to model the experimental results with a good deal of confidence. 
Now it is necessary to discuss how these benchmarks are chosen and how they directly apply to this project. Much of the CAS analysis performed hinges on the overall ability of MCNP5 to correctly model criticality events. More specifically this code must accurately account for fission of ${ }^{235} \mathrm{U},{ }^{239} \mathrm{Pu}$, and ${ }^{252} \mathrm{Cf}$ and the resulting myriad of interactions that the particles may undergo after fission. The following criteria should be used to judge the applicability of a benchmark to what is being modeled:

- Reflection

- Energy range of the particle(s) being investigated

- Geometry

- Magnitude of the fluence being investigated

- Material compositions

There is no way to assert that if $\mathrm{x}$ number of the above criteria are met then the benchmark is relevant. What can be said is that the more closely the benchmark resembles what is being investigated, the easier it will be to argue that it is relevant.

A set of evaluated benchmarks was selected from the fundamental physics section of the IHECSBE. This set of experiments is designated in the handbook as FUND-NISTCF-MULT-FISS-001 (Kim, 1995). These experiments were performed from 1989-1990 at the National Institute of Standards and Technology facility in Gaithersburg, Maryland. This research was performed using a spherical stainless steel container and fission chambers to investigate discrepancies in neutron transport being observed in subcritical assemblies. A lightly encapsulated ${ }^{252} \mathrm{Cf}$ neutron source was placed at the center of the spherical shell of Stainless steel. Absolute fission rates of ${ }^{235} \mathrm{U},{ }^{239} \mathrm{Pu},{ }^{238} \mathrm{U}$, and ${ }^{237} \mathrm{~Np}$ deposits in fission chambers positioned outside the spherical container were measured. The measurements were performed for spherical containers of three different diameters; 
3-in., 4-in. and 5-in., with and without water in the spherical container. So it is clear that these experiments thoroughly investigate fission in all of the isotopes used in the CAS models. To replicate the results of the benchmarks, MCNP must track particles through several materials before a normalized fission rate is calculated. It is asserted that if the model created in MCNP5 can accurately replicate these experimental results, then MCNP5 is valid for use in this analysis for the design of a CAS.

It was selected to use 3-in., 4-in. and 5-in spheres filled with water and the same set repeated for the sphere filled with air. Absolute fission rates were obtained for all four isotopes listed above, but only the ${ }^{235} \mathrm{U}$ and the ${ }^{239} \mathrm{Pu}$ results are relevant to these models and hence the validation. Each case was modeled with the same version of MCNP5 used for the CAS analysis. A diagram of the general setup for the NIST experiments is shown in Figure 4. 


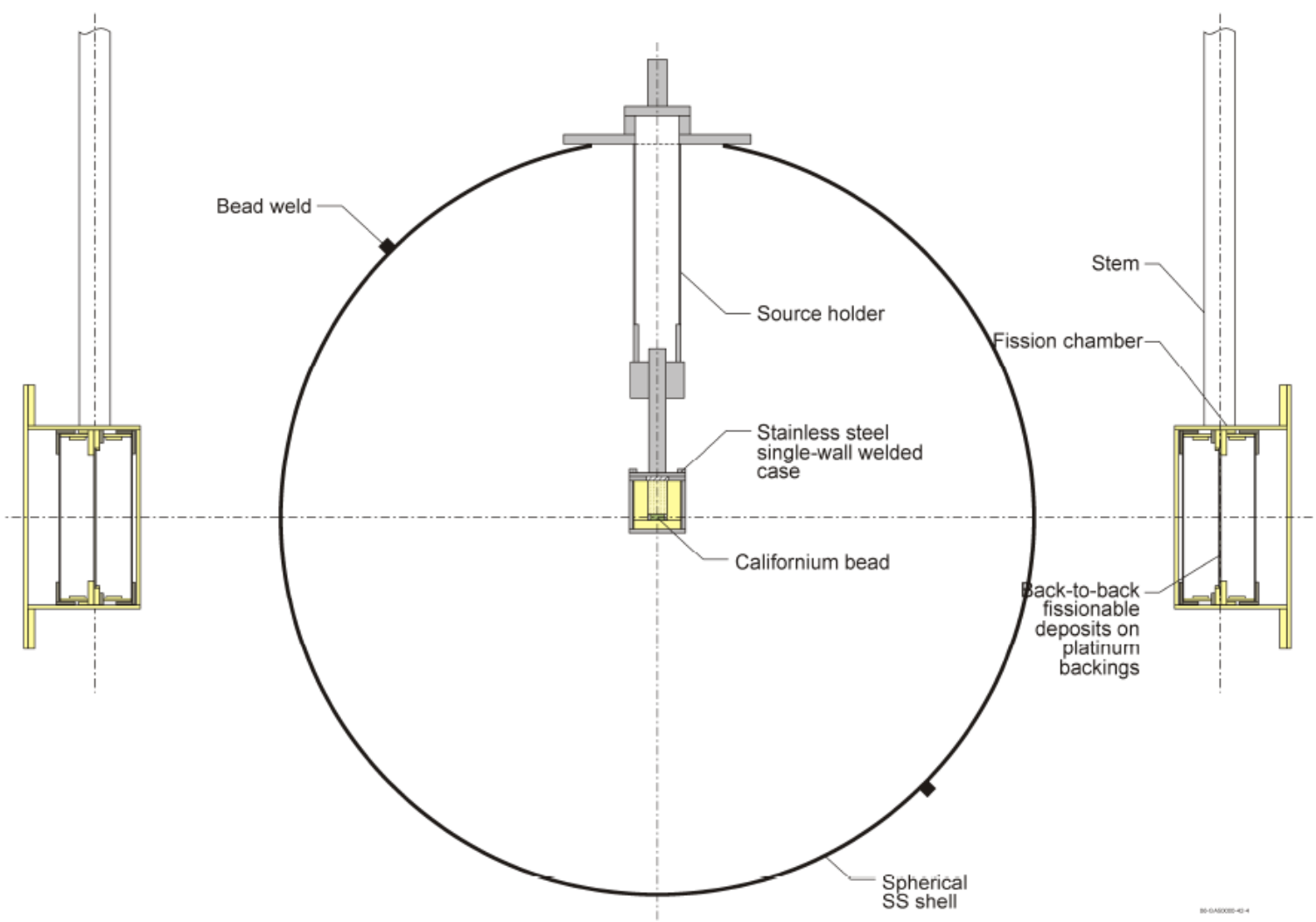

Figure 4. Experimental setup for the NIST Sphere Benchmarks

The Spherical Stainless Steel Shell had a diameter of 3, 4, or 5 inches. The detectors remained in the same location for both the 3- and 4-inch Shells -distance from the source was unchanged. In the 5-inch experiments, to facilitate the increase in radius of the shell, the detectors had to be moved 0.75 inches outward. To keep the source at mid-plane with the fission chambers for each shell size, the source holder was either lengthened or shortened accordingly. For the water-filled experiments, the sphere shown above was completely filled with room temperature water. These are the only geometric parameters altered for the experiments. 
Table 1 gives the experimental results for the normalized fission rates in barns, as well as the associated relative error. MCNP5 will output a variety of cross section values including single group effective fission cross-sections in barns. That cross-section value multiplied by the fluence striking the fission detector yields the benchmark designated Fission Rate in barns shown in Table 1. Further details on how the Fission Rate was calculated can be found in (Kim, 1995).

The implications of having data for both water-and air-filled spheres are important to note. It is imperative for proving validity that the code is able to accurately account for the scattering and fission of neutrons. Demonstrating that MCNP5 can track neutrons through a strong scattering medium such as water, for all the isotopes involved in this analysis proves the code will perform as needed. The air-filled sphere data is important because the spectrum of the neutrons is much harder than that of the waterfilled experiments. Showing that MCNP5 can track and account for fission of both thermal and fast neutrons is crucial to the dependability of the CAS analysis.

Table 1. Experimental normalized fission rates for ${ }^{235} \mathrm{U}$ and ${ }^{239} \mathrm{Pu}$

\begin{tabular}{|l|c|c|c|c|}
\hline Isotope & Condition & Sphere & $\begin{array}{c}\text { Fission Rate } \\
\text { (barns) }\end{array}$ & $\begin{array}{c}\text { Relative Error } \\
(\mathbf{\%})\end{array}$ \\
\hline U-235 & dry & 3-inch & 1.278 & 1.6 \\
\hline & dry & 4-inch & 1.279 & 1.6 \\
\hline & dry & 5-inch & No Results & \\
\hline & wet & 3-inch & 19.6 & 1.7 \\
\hline & wet & 4-inch & 45.7 & 1.7 \\
\hline & wet & 5-inch & 72.2 & 1.7 \\
\hline & & & & 1.5 \\
\hline Pu-239 & dry & 3-inch & 1.916 & 1.5 \\
\hline & dry & 4-inch & 1.924 & 1.5 \\
\hline & dry & 5-inch & No Results & 1.5 \\
\hline & wet & 3-inch & 36.7 & 1.5 \\
\hline & wet & 4-inch & 82.3 & \\
\hline & wet & 5-inch & 125.5 & \\
\hline
\end{tabular}


Table 2 shows the results found from modeling the experiment in MCNP5 and the percent that this computational result differs from the experimental value.

Table 2. MCNP5 Computational results with comparison to experimental results

\begin{tabular}{|l|c|c|c|c|c|}
\hline Isotope & Condition & Sphere & $\begin{array}{c}\text { Fission } \\
\text { Rate } \\
\text { (barns) }\end{array}$ & $\begin{array}{c}\text { MCNP5 } \\
\text { Results }\end{array}$ & $\begin{array}{c}\text { Variance from } \\
\text { Experimental } \\
\text { (\%) }\end{array}$ \\
\hline U-235 & dry & 3-inch & 1.278 & 1.280 & 0.186 \\
\hline & dry & 4-inch & 1.279 & 1.281 & 0.175 \\
\hline & wet & 3-inch & 19.6 & 20.237 & 3.250 \\
\hline & wet & 4-inch & 45.7 & 46.245 & 1.194 \\
\hline & wet & 5-inch & 72.2 & 72.536 & 0.465 \\
\hline Pu-239 & dry & 3-inch & 1.916 & 1.850 & -3.457 \\
\hline & dry & 4-inch & 1.924 & 1.847 & -4.027 \\
\hline & wet & 3-inch & 36.7 & 37.339 & 1.742 \\
\hline & wet & 4-inch & 82.3 & 81.753 & -0.664 \\
\hline & wet & 5-inch & 125.5 & 124.669 & -0.662 \\
\hline
\end{tabular}

The requirement at PNNL for dose modeling accuracy is only plus or minus 20 percent of the accepted values. Table 2 clearly shows that MCNP5 exceeds this requirement. Table 2 shows the largest variance is -4.027 percent in the ${ }^{239} \mathrm{Pu}$ dry 4 -inch sphere model. The results obtained with MCNP5 clearly show that MCNP5 can accurately model fission of ${ }^{235} \mathrm{U},{ }^{252} \mathrm{Cf}$, and ${ }^{239} \mathrm{Pu}$. It is also clear that MCNP5 models neutron transport through strong scattering media substantially well. 


\section{Design and model the criticality alarm system for the facility;}

\section{Subtask 1 - Finding the MAC including fluence and spectrum}

Table 3 shows the results for the minimum accident scenarios that contained Plutonium. The Uranium accident scenarios were not included because the Plutonium accidents were found to generate fewer neutrons. These results were obtained using MCNP5 with F6 dose tallies. The inputs for all the Plutonium scenarios used to determine the MAC are listed in Appendix F. Looking at Table 3 it makes sense based on the shielding properties of neutrons that a water moderated system shields the most neutrons. Table 3 shows the minimum neutron fluence for RPL, which was found to be 3.94E15 (n/cm^2-s) for the MAC. This fluence was the result of an optimally moderated bare Plutonium and water sphere. This scenario had the largest portion of gamma-rays contributing to the dose limit. For reference the RPL minimum accident of concern MCNP5 input file is included in Appendix F.

Table 3. Plutonium minimum accident scenario data

\begin{tabular}{|c|c|c|c|}
\hline $\begin{array}{c}\text { Accident Scenario } \\
\text { for Optimally } \\
\text { Moderated 1kg } \\
\text { Pu-239 and H2O } \\
\text { Spheres }\end{array}$ & $\begin{array}{c}\text { Neutron } \\
\text { Dose per } \\
\text { Source } \\
\text { Particle } \\
\text { (MeV/g) }\end{array}$ & $\begin{array}{c}\text { Photon } \\
\text { Dose per } \\
\text { Source } \\
\text { Particle } \\
\text { (MeV/g) }\end{array}$ & $\begin{array}{c}\text { Total Source } \\
\text { Neutrons } \\
\text { Required to } \\
\text { Reach the } \\
\text { MAC }\end{array}$ \\
\hline Unreflected & $1.34 \mathrm{E}-08$ & $8.92 \mathrm{E}-08$ & $3.94 \mathrm{E}+15$ \\
\hline Water Reflected & $2.94 \mathrm{E}-10$ & $8.84 \mathrm{E}-08$ & $4.90 \mathrm{E}+15$ \\
\hline Iron Reflected & $3.86 \mathrm{E}-09$ & $4.74 \mathrm{E}-09$ & $5.06 \mathrm{E}+16$ \\
\hline
\end{tabular}

When tracking photons in MCNP5, the user must designate the appropriate crosssections for both neutrons and for photons. Cross-sections must also be included for photonuclear effects if the physics card does not explicitly disable them. Correctly defining the cross-sections and the physics options are not trivial exercises. Using the 
wrong libraries or incorrectly tracking the particles can result in much different answers. Chapter II of the MCNP5 manual goes into greater detail on this matter so refer to it for specific guidance (X-5 Monte Carlo Team, 2003).

It is important to remember that the F6 tally output is normalized to per starting particle. This means that the branching ratios of the fission events taking place are not represented in the tallied quantities. So they must be included to determine the Total Source Neutrons Required to Reach the MAC quantity listed in Table 3. For example, a non-kcode calculation, for californium-252 emulates the fission energy spectrum but it does not account for the average number of neutrons emitted per fission (nu). Not including the correct branching information would result in source numbers that were at least a factor of $\mathrm{Nu}$ too high, which could invalidate the calculated detector coverage.

Also it is important to remember that MCNP5 does not track delayed photons. It is essential to include the delayed photon contribution because it ultimately lowers the source term needed to reach the minimum dose level. Omitting the delayed fraction is then non-conservative and should be addressed. Depending on how much time is available, burn up calculations can be performed to determine the isotopics as a function of accident history. Then another MCNP5 input can be constructed that tracks only the resultant photon contribution from these delayed products. It was attempted to get this dose contribution for the delayed fraction but the total delayed contribution never amounted to more than roughly 3.5 percent of the total dose contribution. Several engineers felt that 3.5 percent was too small of a delayed fraction to guarantee conservatism. It was advised to assume a delayed dose contribution of 7 percent to be sure. It is unlikely that the delayed photon contribution is that high. Because there was 
not great confidence in the numbers found with MCNP5, the decision was made to error on the side of conservatism.

\section{Subtask 2 - Identify potential locations for detector placement}

An analysis of the detector placement in RPL was performed using the Criticality Slide Rule (UNCSR) and an assumed minimum detectable dose value. To find the minimum detectable dose value, assumptions had to be made for the following quantities:

1. Average neutron fission energy of $2.1 \mathrm{MeV}$ for ${ }^{239} \mathrm{Pu}$

2. Average value of interceding shielding of 8 inches of concrete

3. Minimum detectable fluence of $1 \mathrm{e} 5$ neutrons

Assumption 1 was made because the only fissile material that the MAC contained for RPL was ${ }^{239} \mathrm{Pu}$. Assumption 2 was found by looking at the materials between the accident locations and the detectors. The actual shielding values are different for every MAC location but an average value was used to save time.

The final assumption for the minimum detectable fluence was not as straightforward as the first two. At this point in the analysis, detector count rates, efficiency, and spectral dependence were not known for the system. Not knowing these parameters for the detectors to be used in the facility made choosing the minimum detection value difficult. Because no alternative was found a typical value was assumed for a similar detector type found on the General Electric (GE) website for a Reuter-Stokes $\mathrm{BF}_{3}$ tube. The GE tube value had a lower detection threshold than the 1e5 fluence chosen for this system but the $\mathrm{BF}_{3}$ tubes that were available for this CAS were legacy tubes, 
some over 40 years old. To compensate for the age, the decision was made to increase the GE minimum detection value by a factor of 100 .

Using Equation 1 and the assumed parameters discussed above, the final equivalent minimum detectable dose was calculated to be $0.028 \mathrm{rad}$. The resulting dose reduction factor for 8 inches of concrete was 0.70 . This yielded a final dose of 0.02 rad at a distance of 200ft. An arc was traced over facility drawings using the correctly scaled distance for each MAC. Figure 5 and Figure 6 show the arcs for the basement and first floors of RPL, respectively.

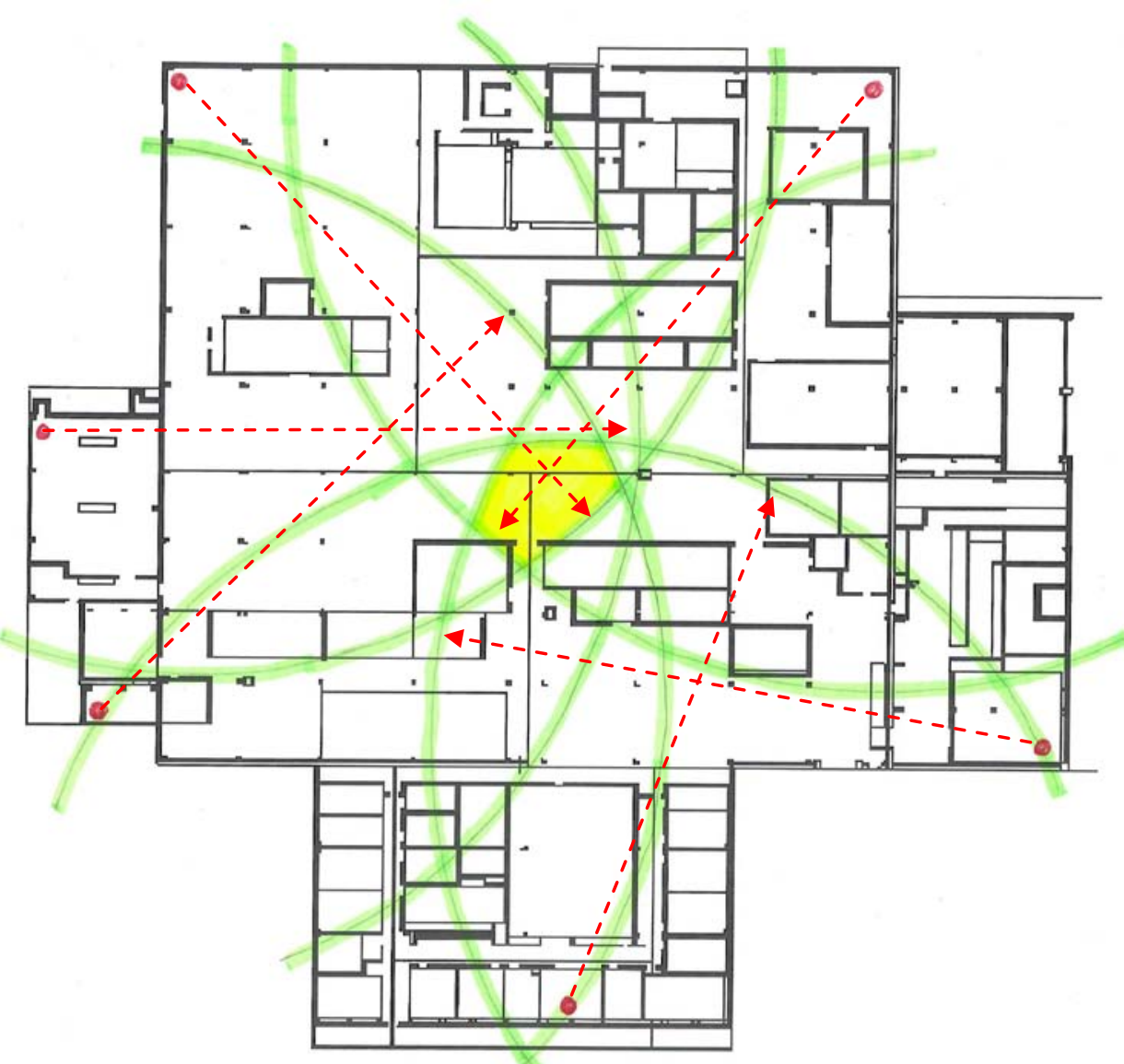

Figure 5. 0.02 rad arcs for the outlying MAC's in the basement of RPL 


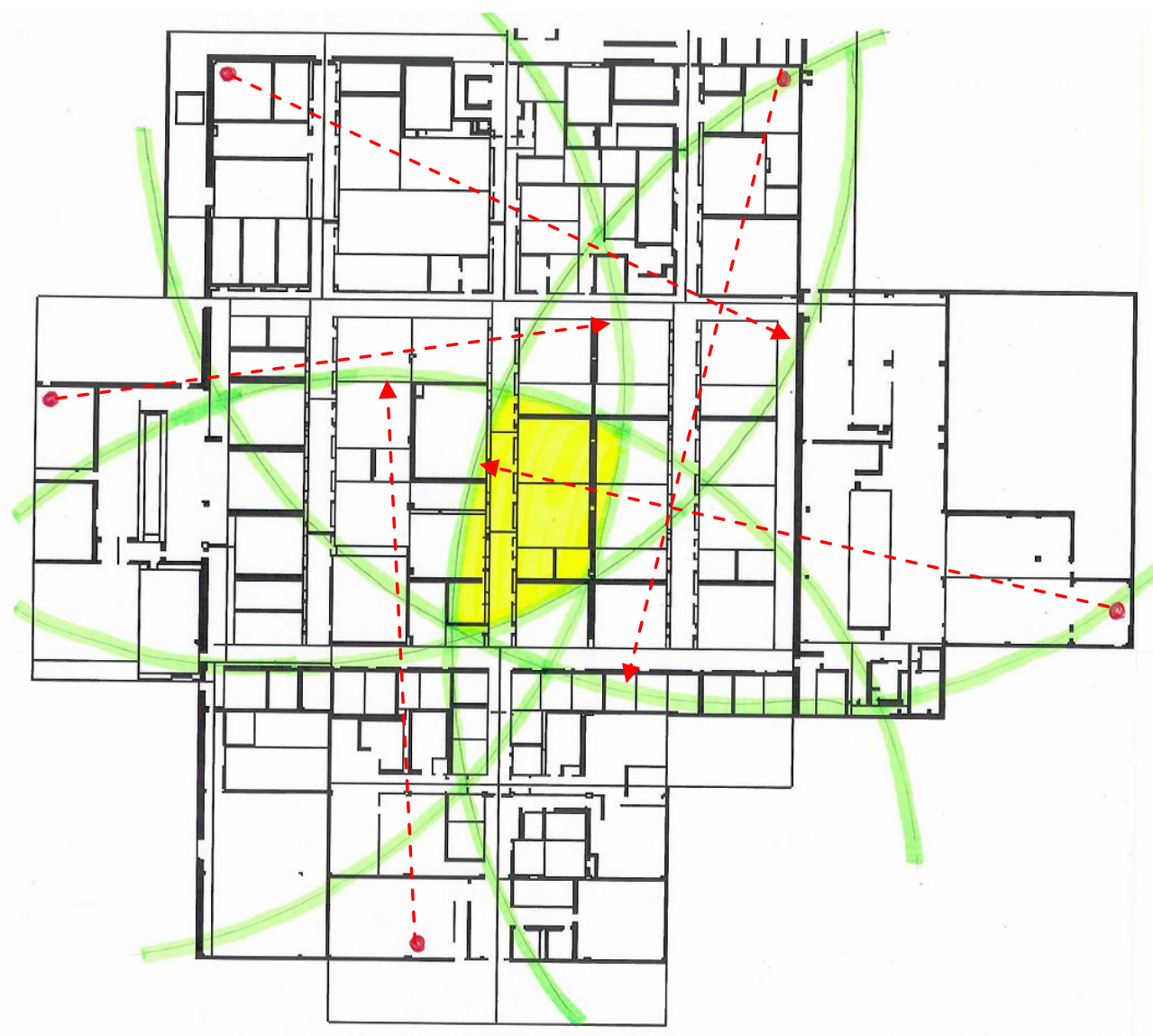

Figure 6. 0.02 rad arcs for the outlying MAC's in the first floor of RPL

The green arcs are the assumed minimum detectable distances for the specified parameters. The red dots are the MAC locations that correspond to the arcs. The highlighted areas represent the greatest number of arc unions on that floor. The most outlying MAC locations were chosen because they represent the most challenging detection locations. Comparing Figures 5 and 6 to Figures 7 and 8 shows how good of an approximation the Criticality Slide Rule provides. The actual detector locations for the basement are very near the highlighted areas. The first floor is slightly less accurate but the majority of the actual detector locations are within a small distance of highlighted 
area. It can be concluded from Figures 5 and 6 that the assumed minimum detectable distance was slightly conservative, but still relatively accurate for predicting the detector coverage in the facility.

\section{Subtask 3 - Modeling the facility}

\section{a. Assumptions/omissions/simplifications}

All of the assumptions made for the CAS design of RPL are given in Appendix E. One major assumption for the RPL facility model was to standardize the fill material for the different spaces in the building. As mentioned in the Design Methodology section to be conservative, the material located inside of the facility must be included in the model. The method used for the RPL project was to make fill approximations based on facility inspections. The following classifications were made based on the average contents of the spaces in RPL, Empty Rooms, Full Office, Full Laboratory, Half Full Office and Half Full Laboratory. The contents of each classification are given in subtask c. These fill definitions were used to characterize the spaces within the facility.

To make the application of these fill definitions easier, the items located in the requisite space, i.e., desks, glove boxes, bookshelves, are homogenized for material definition. What this means is that the volumes of the corresponding items are conserved, but the items do not retain their geometric shape. The corresponding number densities for each item desks, glove boxes, bookshelves, etc., are all found independently and then added together to make the final appropriate room fill definitions. 


\section{b. Dimensions}

Figures 7, 8, and 9 are floor layouts for RPL generated with the program Vised mentioned earlier. The red dots represent where each MAC was placed for the simulations. The green squares represent the existing detectors. Figure 9 shows the $2^{\text {nd }}$ floor of RPL. There is only one laboratory on that level and the rest of the space in the northwest corner is taken up by offices. On the east side of that same figure is the top of High Level Radiochemistry Facility (HLRF). At that altitude HLRF is only open space and structural material.

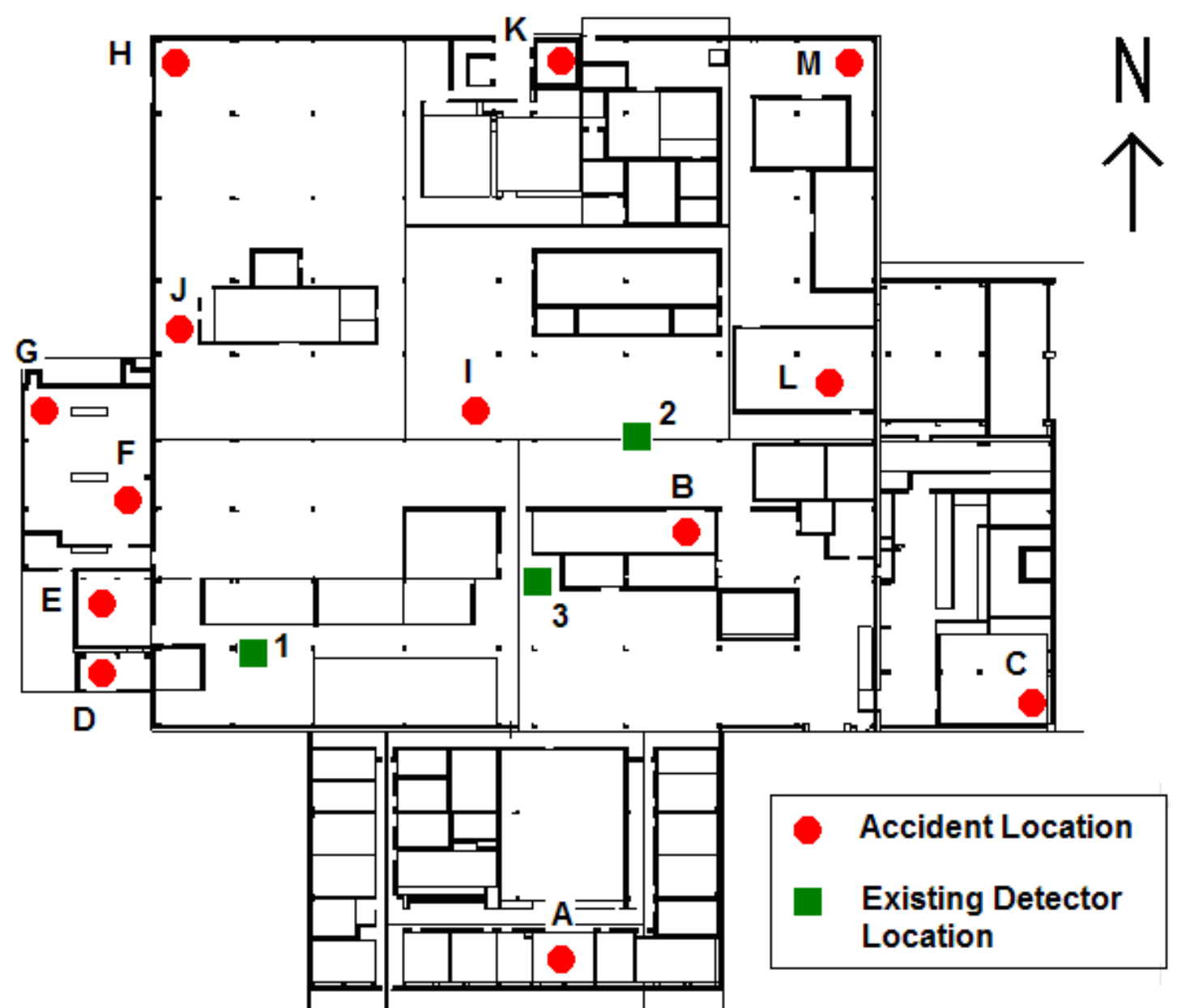

Figure 7. RPL Basement shown with modeled accident locations and the existing detector placement 


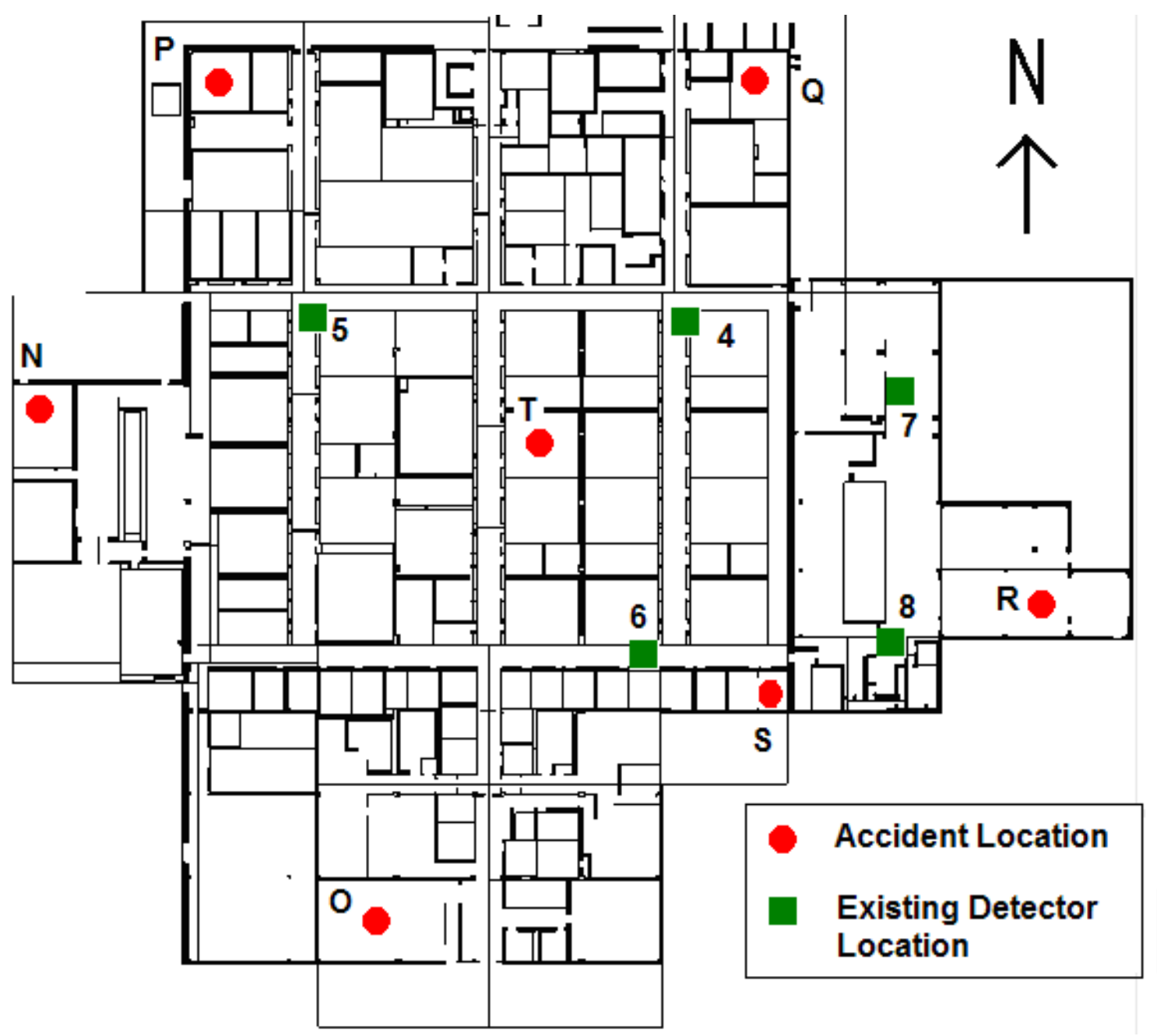

Figure 8. RPL First floor shown with modeled accident locations and the existing detector placement 


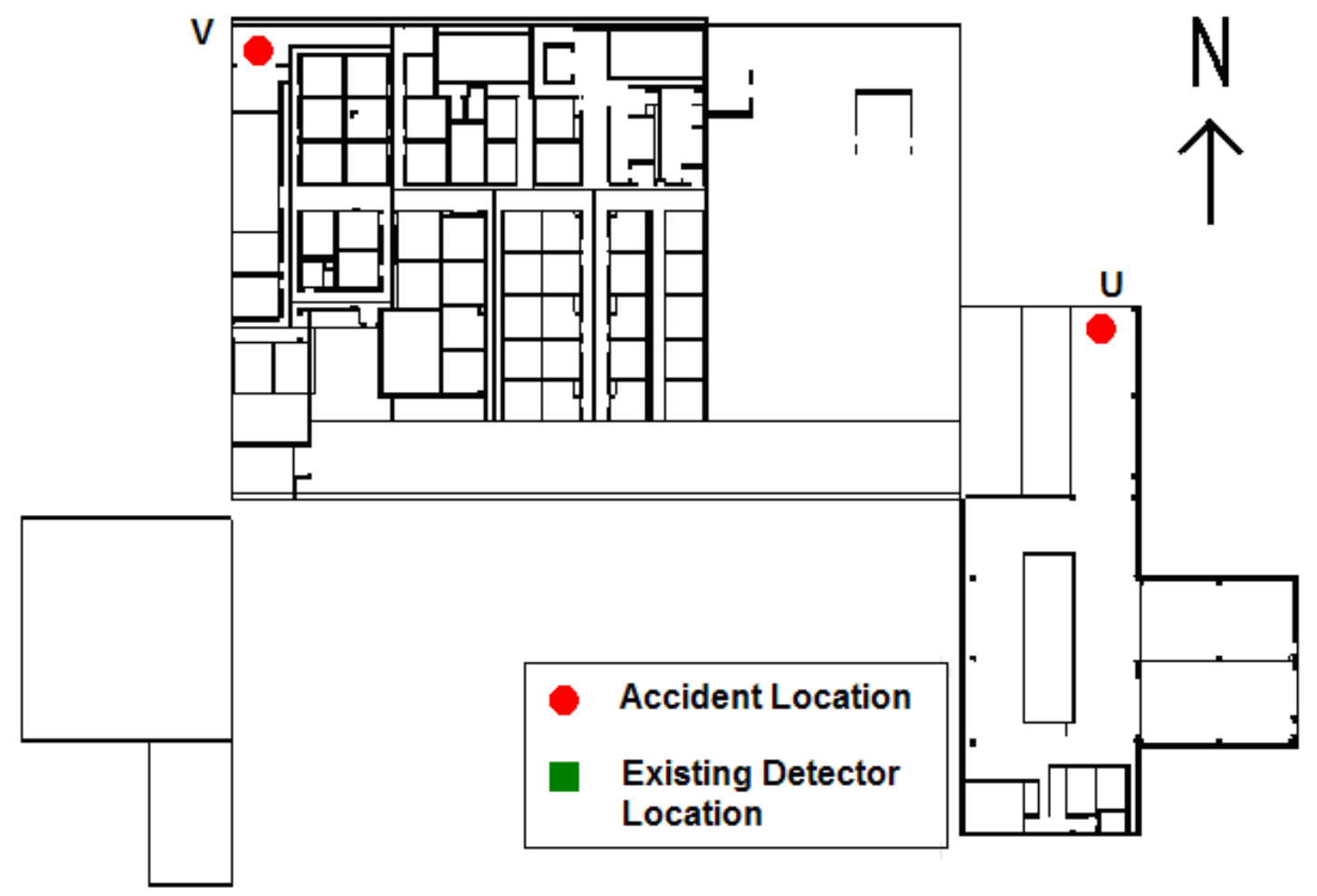

Figure 9. RPL Second floor layout

\section{Facility Description}

Building 325 houses the Radiochemical Processing Laboratory (RPL). It is located in the 300 Area of the Hanford Site in Washington State. The building is used to conduct radiochemical research. RPL contains laboratories and other facilities designed for work with non-radioactive materials, microgram-to-kilogram quantities of fissionable materials, and up to mega-curie quantities of other radionuclides. It is a Class 2 nuclear facility.

The RPL consists of a central portion, a south (front) wing, east and west wings. The central portion of the building contains over 100 laboratories and offices on three floors. The second floor and basement contain mechanical areas such as supply fans, steam lines, etc. The south wing (two floors) contains offices, a conference room, a 
machine shop, a lunchroom, and rest rooms. The east wing (one floor) houses the highlevel radiochemistry facility (HLRF), truck lock, and manipulator repair area. The west wing (one floor) houses the shielded analytical laboratory (SAL).

\section{c. Materials Used in Computational Models}

A note of caution: it was not determined until late in the analysis that the cinderblock walls of RPL were not hollow. During a QA mandated facility walkdown, it was discovered that the cinderblock walls were actually back-filled with concrete. Because this finding increases the amount of intervening material in the building, the models had to be updated and restarted. Fortunately the mistake was found early enough to not adversely impact the schedule.

\section{Empty Rooms}

Any room found to be "empty" was filled with air. The chemical composition of air is found in Appendix C. "Empty" was defined in the following way. Hallways were considered empty, including entryways and any space that looked to be wide open. Rooms void of furniture were likewise modeled as empty. Laboratories with empty cabinets built into the sides were modeled as empty. Bathrooms were modeled as empty.

\section{Full Office}

Offices that were fully functional, filled with a desk, full bookshelves, file cabinets, etc., were modeled as "full offices." The justification and calculation of the material composition of a "full office" is provided in Appendix C. The office material 
mixture was based on the dimensions of room 113 in the RPL. This room has dimensions of $350 \mathrm{~cm}$ by $313.7 \mathrm{~cm}$ by $442 \mathrm{~cm}$. It was assumed that the "typical” full office contained three full bookshelves, one full desk, and one full file cabinet. The measured dimensions of a typical bookshelf are $208.3 \mathrm{~cm}$ by $91.5 \mathrm{~cm}$ by $30.5 \mathrm{~cm}$. The measured dimensions of a typical file cabinet are $152.4 \mathrm{~cm}$ by $38.1 \mathrm{~cm}$ by $66 \mathrm{~cm}$. The desk was modeled as three file drawers with dimensions $91.5 \mathrm{~cm}$ by $38.1 \mathrm{~cm}$ by $66 \mathrm{~cm}$. It was assumed that all the bookshelves and file drawers (cabinets included) were full of paper. According to (Reppond, 1977), wood has a material composition of 44.44 weight percent carbon, $6.22 \%$ hydrogen, and $49.34 \%$ oxygen with an overall density of $1.0 \mathrm{~g} / \mathrm{cm}^{3}$. The density assumed for the wood is intentionally high to ensure that even water-logged wood is accounted for. Appendix C provides the calculations for the "full-office" homogeneous material. The "half-filled office" was assumed to have one half the material in the room.

\section{Full Laboratory}

Laboratories were judged, upon personal inspection, to be “empty,” “full,” or "sparse.” The justification and calculation of the material composition of a "full laboratory” is provided in Appendix C. The laboratory material mixture was based on the dimensions of the room 312 in the RPL. This room has dimensions of $802.64 \mathrm{~cm}$ by $601.98 \mathrm{~cm}$ by $441.96 \mathrm{~cm}$. There is also a wall section that cuts into the total volume of the room with dimensions $220.98 \mathrm{~cm}$ by $60.96 \mathrm{~cm}$ by $441.96 \mathrm{~cm}$. It was assumed that the “typical” full laboratory contained four full bookshelves, four full file cabinets, and one glove box with six lead bricks. To represent the various instruments in the room, a 2-cm 
thick sheet of stainless steel and a 2-cm thick sheet of glass was placed in the room. To represent all the tables, cabinets, and desktops, an 16-cm thick sheet of wood was placed in the room. The same dimensions for the desk, bookshelf, and file cabinet that were used in the office calculation were used in this calculation. The glove box has 6 panels of stainless steel and 4 panels of glass. The homogeneous material composition for the "full laboratory” is calculated in Appendix C.

\section{Sparse Laboratory}

The justification and calculation of the material composition of a "sparse laboratory” is provided in Appendix C. The same dimensions were used that are described in "full laboratory." For the sparse laboratory, it is assumed that there is onehalf the total amount of material in full laboratory. The homogenous material composition for the "sparse laboratory” is calculated in Appendix C.

\section{Additional Materials}

The rest of the materials used in the models were all approximated using existing published information on typical densities and compositions. For example the MCNP5 material card for the structural concrete used to model RPL is shown in Figure 10. The

densities listed in Figure 10 are atom fractions with the units of atoms per barn centimeter. 
c Calculated Density -2.3g/cc Standard Concrete

\begin{tabular}{|c|c|c|}
\hline $1001.66 \mathrm{c}$ & -0.022100 & \$ H Concrete (Ordinary) \\
\hline $6000.66 c$ & -0.002484 & \$C \\
\hline $8016.66 c$ & -0.574930 & $\$ O$ \\
\hline 11023.66c & -0.015208 & $\$ \mathrm{Na}$ \\
\hline $12000.66 c$ & -0.001266 & \$ Mg \\
\hline $13027.66 \mathrm{c}$ & -0.019953 & $\$ \mathrm{Al}$ \\
\hline $14000.60 \mathrm{c}$ & -0.304627 & $\$ \mathrm{Si}$ \\
\hline $19000.66 c$ & -0.010045 & \$ K \\
\hline $20000.66 c$ & -0.042951 & \$ Ca \\
\hline $26000.55 c$ & -0.006435 & $\$ \mathrm{Fe}$ \\
\hline
\end{tabular}

Figure 10. Standard concrete material definition of $2.3 \mathrm{~g} / \mathrm{cc}$ density.

\section{d. Detectors}

The detectors used in RPL are $\mathrm{BF}_{3}$ gas proportional detectors, which have active dimensions of $21.6 \mathrm{~cm}$ by $1.27 \mathrm{~cm}$. The Boron is $95 \%$ enriched ${ }^{10} \mathrm{~B}$ and the pressure of the gas is approximately $0.70 \mathrm{~atm}$. Each $\mathrm{BF}_{3}$ is surrounded by $5.75 \mathrm{~cm}$ by $21.6 \mathrm{~cm}$ Right cylinder of Polyethylene. The calculated efficiency of the detectors was on average 7 percent. These detectors are designed such that when a failure of the tube occurs, it causes an "alarm" signal. This failed alarm mode is important when the accident scenarios are maximized to determine the 12 rad boundary; another regulatory concern out of the scope of this document.

\section{e. Source}

To model the MAC discussed above for RPL, a variety of MCNP5 features were used. The SDEF card was used with the coefficients corresponding to a Watt fission spectrum for thermal fission of ${ }^{239} \mathrm{Pu}$. To get the appropriate geometric distribution, the source was smeared isotropically throughout a sphere that contained the correct volume of water. Depending upon the location of the accident in the building, the number of 
particles that were needed to get acceptable statistics varied considerably. The smallest number was only $10^{5}$ but the largest number was $10^{10}$.

\section{f. Tallies}

The only technically useful information that is warranted here is to show how the F2 surface flux approximation tally differed from the F4 cell weighted flux tally. Otherwise the tallies are thoroughly discussed in the MCNP5 manual (X-5 Monte Carlo Team, 2005).

It was found that the F2 tally generally over reported the surface flux for cylindrical and spherical tally surfaces more than it did for planar surfaces. How much the F2 tally over reported the flux varied from almost none to as much as 10 percent. Whether the F2 tally is over reporting fluences or the F4 tally is under reporting is not known. To maintain conservatism, the decision was made to use only the F4 cell tallies because there is no large angle approximation used in its computation, and it reported the lowest fluences of the two tallies.

\section{g. Accident Locations}

All that must be done is to prove that the alarm system can see an accident anywhere in the facility, plausible or not. So enough accident locations must be selected to show the total facility coverage. Generally all the corners, all of the heavily shielded areas, and all of the hot cells should have accidents modeled there. To show complete coverage for RPL, a total of 24 accident locations were chosen. The number of accident locations chosen to prove coverage is dependent on the size of the facility and the 
available computational capability. The 24 accident locations chosen for RPL represent all of the most difficult to detect areas for the CAS. Intuition should be used in the determination of these locations. Areas with large amounts of intervening shielding and places that are the furthest from the detectors will commonly be the hardest to see. Figures 7, 8, and 9 show the modeled accident locations for RPL.

\section{Subtask 4 - Find count rates for the criticality detectors for known conditions}

The ratio of particles that result in a count is the actual efficiency of the detector. It makes little difference whether the efficiency is good or bad; what matters is that the efficiency is known, and known with confidence. PNNL operates a calibration lab where all of the NCD are routinely recalibrated and checked for problems. Their procedure for NCD calibration utilizes a neutron counting well with a ${ }^{252} \mathrm{Cf}$ neutron source placed in a motorized elevator. More importantly their procedure uses a set of three $\mathrm{BF}_{3}$ tubes that were sent to the National Institute of Standards and Technology (NIST), where count rates for each tube were found at known dose rates with a high degree of certainty.

The NIST calibrated $\mathrm{BF}_{3}$ tubes are used to find an elevation for the source in the well that yields a count rate that corresponds to the known dose rate. The NCD to be calibrated is then placed in the well, and its count rate obtained. If this count rate is not within 10 percent of the golden count rate, the gain or the dwell time is changed until it is. The count rate data for each NCD is recorded and plotted over time to show any underlying trends and how the tubes are progressing over time. It was found that the average count rate for all three golden $\mathrm{BF}_{3}$ tubes was 563 counts per second at a distance

of 1.61 meters from the source at 9:15am of March $3^{\text {rd }} 2009$. This information is used to recreate the calibration setup in MCNP5 in subtask 5 so that the DCTE can be found. 


\section{Subtask 5 - Modeling the Calibration/Counting Setup to find the Detector Cell Tally Efficiencies}

The data found in subtask 4 was used to create an accurate model of the calibration setup so that the DCTE could be found. Table 4 shows decay corrected activity data for the ${ }^{252} \mathrm{Cf}$ NCD calibration source that was used in the model.

The neutron source was modeled using the spontaneous fission spectrum of ${ }^{252} \mathrm{Cf}$ provided by MCNP5 and the decay corrected activity for the simulated source weight. In MCNP5, the source weight is used to scale the tallied results to a real world yield. The same geometric model and F4 cell flux tally of the NCD used in the facility model was placed in the calibration mock-up.

Table 4. ${ }^{252} \mathrm{Cf}$ calibration source information

\begin{tabular}{|l|c|c|c|c|}
\hline \multicolumn{1}{|c|}{ Date } & $\begin{array}{c}\text { Seconds } \\
\text { Elapsed }\end{array}$ & $\begin{array}{c}\text { Activity } \\
\text { (Ci) }\end{array}$ & Activity $\mathbf{( B q )}$ & $\begin{array}{c}\text { Neutrons per } \\
\text { second }\end{array}$ \\
\hline $\mathbf{9 / 2 7 / 1 9 9 5}$ & $0.0000 \mathrm{E}+00$ & 0.6880 & $2.5456 \mathrm{E}+10$ & $2.9340 \mathrm{E}+09$ \\
\hline $\mathbf{3 / 4 / 2 0 0 8}$ & $3.9243 \mathrm{E}+08$ & 0.0264 & $9.7840 \mathrm{E}+08$ & $1.1277 \mathrm{E}+08$ \\
\hline $\mathbf{1 / 1 9} / \mathbf{2 0 0 9}$ & $4.2016 \mathrm{E}+08$ & 0.0210 & $7.7713 \mathrm{E}+08$ & $8.9569 \mathrm{E}+07$ \\
\hline $\mathbf{3 / 9 / 2 0 0 9}$ & 424396800 & 0.0203 & $7.5028 \mathrm{E}+08$ & $8.6475 \mathrm{E}+07$ \\
\hline
\end{tabular}

The F4 tally indicated that 1440 particles strike the high density polyurethane (HDPE) collar per second for the identical calibration scenario described in task 4.

Dividing the golden count rate by this tallied fluence gives a DCTE of 0.39 percent of the simulated particles entering the HDPE collar result in a real world count. 


\section{Subtask 6 - Adjust the value for the detector minimum trip setting}

The count rate found by NIST for the set of three $\mathrm{BF}_{3}$ tubes corresponds to a dose rate in free air of $80 \mathrm{mrem} / \mathrm{hr}$. This was the dose rate and ultimately the count rate used for the trip setting of the NCD in RPL. To the best of the knowledge of the engineers at PNNL, there has never been a false alarm in the facility. This fact was important to note because the facility has been in operation for over 50 years. This established track record was a great help for basing the trip setting high enough to avoid false alarms. It is unlikely to ever see a natural or cosmic background source that would approach even ten percent of the required 563 counts per second alarm setting. What may be plausible is that man-made sources could potentially reach this trip setting. To avoid potential manmade triggered false alarms, every effort was made to not decrease the trip setting.

Each facility will present its own challenges but it is this balance between detection and false alarms that is the most likely to cause problems for the engineer. RPL has a large amount of concrete, both regular and Barytic. RPL also has dozens of heavily shielded hot cells spread throughout the building. All of this material adds to the challenge of detecting an accident in any location. With diligence, effective coverage can be obtained with trip settings that are high enough to avoid almost all false alarms.

\section{Subtask 7 - Verify that adequate coverage has been obtained at the new minimum trip setting}

Once the initial accident modeling runs were completed and the trip setting agreed upon, the rest of the building inputs were run. Table 5 shows the final coverage for RPL. Table 6 shows the room locations of the accidents. These are the same accidents shown

on the facility models in Figure 7, Figure 8, and Figure 9. Dividing the trip setting listed 
in subtask 5 of 563 counts per second by the DCTE found in subtask 4 of 0.390 yields the computational equivalent of an alarm. Therefore the minimum F4 tally value that represents an alarm is 1440 particles. It can be seen in Table 8 that all the reported values are greater than the 1440 particles needed to trip a detector. 
Table 5. Detector counts for each MAC location in RPL

\begin{tabular}{|c|c|c|c|c|c|c|c|c|c|}
\hline $\begin{array}{l}\text { Accident } \\
\text { Location }\end{array}$ & Floor & NCD 1 & NCD 2 & NCD 3 & NCD 4 & NCD 5 & NCD 6 & NCD 7 & NCD 8 \\
\hline Rm203 & 1 & $1.80 \mathrm{E}+06$ & & & $2.37 \mathrm{E}+05$ & $1.14 \mathrm{E}+05$ & $4.66 \mathrm{E}+07$ & & \\
\hline $\begin{array}{l}\text { Lunch } \\
\text { Room }\end{array}$ & 1 & $2.83 E+07$ & & $8.89 \mathrm{E}+05$ & & $6.69 \mathrm{E}+06$ & & $1.10 \mathrm{E}+06$ & \\
\hline $\begin{array}{l}\text { Room } \\
\text { 327A }\end{array}$ & 1 & $1.64 \mathrm{E}+06$ & $6.43 \mathrm{E}+06$ & & $3.33 E+05$ & & $1.34 \mathrm{E}+07$ & & \\
\hline Room 528 & 1 & & $6.90 \mathrm{E}+05$ & & $2.69 \mathrm{E}+07$ & & $2.21 \mathrm{E}+05$ & & $3.16 \mathrm{E}+05$ \\
\hline $\begin{array}{l}\text { HLRF } \\
\text { Truck } \\
\text { Lock }\end{array}$ & 1 & & & & $6.87 \mathrm{E}+06$ & $3.96 \mathrm{E}+06$ & & $2.80 \mathrm{E}+08$ & $2.12 \mathrm{E}+08$ \\
\hline Room 119 & 1 & & & $4.14 \mathrm{E}+06$ & $4.42 E+06$ & $3.49 E+08$ & & $1.41 \mathrm{E}+08$ & \\
\hline Room 410 & 1 & & $1.18 \mathrm{E}+09$ & $1.17 \mathrm{E}+08$ & $1.73 \mathrm{E}+07$ & $1.25 \mathrm{E}+07$ & & & \\
\hline $\begin{array}{l}\text { Mezzanine } \\
\text { Lunch } \\
\text { Room }\end{array}$ & B1 & $6.30 \mathrm{E}+05$ & $6.11 \mathrm{E}+05$ & $9.59 \mathrm{E}+06$ & & $9.49 \mathrm{E}+06$ & & & \\
\hline Room 34 & B1 & $1.52 \mathrm{E}+07$ & $1.32 \mathrm{E}+09$ & $8.27 \mathrm{E}+08$ & & $2.29 E+07$ & & & \\
\hline Room 40C & B1 & & & $3.66 \mathrm{E}+06$ & & $1.26 \mathrm{E}+07$ & & $3.14 \mathrm{E}+08$ & $1.49 \mathrm{E}+07$ \\
\hline $\begin{array}{c}\text { Southwest } \\
\text { of Room } \\
\text { 23B }\end{array}$ & B1 & $3.09 \mathrm{E}+07$ & $2.21 \mathrm{E}+05$ & $2.31 \mathrm{E}+05$ & & $2.40 \mathrm{E}+04$ & & & \\
\hline $\begin{array}{c}\text { West of } \\
\text { Room } 23\end{array}$ & B1 & $9.78 \mathrm{E}+07$ & $4.74 \mathrm{E}+06$ & & & $4.25 \mathrm{E}+04$ & $3.25 E+05$ & & \\
\hline $\begin{array}{c}\text { East side } \\
\text { of Room } \\
32\end{array}$ & B1 & $1.06 \mathrm{E}+08$ & $6.57 \mathrm{E}+07$ & $8.91 \mathrm{E}+06$ & & & $5.68 \mathrm{E}+06$ & & \\
\hline $\begin{array}{l}\text { Northwest } \\
\text { side of } \\
\text { Room } 32\end{array}$ & B1 & $3.02 \mathrm{E}+07$ & $2.37 \mathrm{E}+07$ & $2.63 \mathrm{E}+06$ & & & $2.99 \mathrm{E}+06$ & & \\
\hline $\begin{array}{l}\text { North of } \\
\text { Room } 63\end{array}$ & B1 & $1.34 \mathrm{E}+08$ & & $1.80 \mathrm{E}+07$ & $4.07 E+05$ & & $2.22 \mathrm{E}+07$ & & \\
\hline $\begin{array}{c}\text { Southwest } \\
\text { of Room } \\
57 \mathrm{~W}\end{array}$ & B1 & & $2.78 \mathrm{E}+09$ & $1.10 \mathrm{E}+09$ & $1.20 \mathrm{E}+07$ & & $1.46 \mathrm{E}+07$ & & \\
\hline $\begin{array}{c}\text { West of } \\
\text { Room } 55\end{array}$ & B1 & $3.53 E+08$ & $1.37 \mathrm{E}+08$ & $2.66 \mathrm{E}+07$ & & & $8.12 \mathrm{E}+07$ & & \\
\hline Room 90 & B1 & & $5.58 \mathrm{E}+05$ & $2.02 E+05$ & $2.29 E+05$ & & $1.96 \mathrm{E}+05$ & & \\
\hline Room 48 & B1 & & $1.23 \mathrm{E}+08$ & & $9.86 \mathrm{E}+06$ & & & $1.28 \mathrm{E}+06$ & $2.63 \mathrm{E}+07$ \\
\hline $\begin{array}{l}\text { North of } \\
\text { Room } 52 \\
\end{array}$ & B & & $1.32 \mathrm{E}+06$ & & $4.88 \mathrm{E}+06$ & $1.15 \mathrm{E}+05$ & & & $1.11 \mathrm{E}+06$ \\
\hline $\begin{array}{c}\text { Over } \\
\text { Room 603 } \\
\end{array}$ & 2 & & & & $6.72 \mathrm{E}+08$ & $7.75 \mathrm{E}+07$ & & $7.08 \mathrm{E}+08$ & $4.35 \mathrm{E}+09$ \\
\hline $\begin{array}{c}\text { Over } \\
\text { Room } 327\end{array}$ & 2 & $8.26 \mathrm{E}+05$ & & $2.32 \mathrm{E}+05$ & $3.41 \mathrm{E}+06$ & & $1.05 \mathrm{E}+08$ & & \\
\hline
\end{tabular}

Note: All accident locations had a minimum of 4-detector coverage. B1=First basement floor 1=First floor, $2=$ Second floor 
Table 6. As modeled MAC locations in RPL

\begin{tabular}{|c|c|c|c|c|}
\hline $\begin{array}{l}\text { Accident } \\
\text { Location }\end{array}$ & Floor & Position $X$ & $\mathbf{Y}$ & $\mathbf{Z}$ \\
\hline $\begin{array}{l}\text { (A) Mezzanine Lunch } \\
\text { Room }\end{array}$ & Basement & 1334.6 & -847.5 & 202.5 \\
\hline (B) Room 34 & Basement & 1753.5 & 680.5 & 50.0 \\
\hline (C) Room 40C & Basement & 2882.0 & 40.0 & 50.0 \\
\hline $\begin{array}{l}\text { (D) Southwest of Room } \\
\text { 23B }\end{array}$ & Basement & -202.0 & 171.0 & 50.0 \\
\hline (E) West of Room 23 & Basement & -202.0 & 474.0 & 50.0 \\
\hline (F) East side of Room 32 & Basement & -92.0 & 921.0 & 50.0 \\
\hline $\begin{array}{l}\text { (G) Northwest side of } \\
\text { Room } 32\end{array}$ & Basement & -382.0 & 1141.0 & 50.0 \\
\hline (H) North of Room 63 & Basement & 44.5 & 2577.0 & 50.0 \\
\hline $\begin{array}{l}\text { (I) Southwest of Room } \\
57 \mathrm{~W}\end{array}$ & Basement & 1144.5 & 1028.5 & 50.0 \\
\hline (J) West of Room 55 & Basement & 44.5 & 2338.5 & 50.0 \\
\hline (K) Room 90 & Basement & 1314.5 & 2300.5 & 50.0 \\
\hline (L) Room 48 & Basement & 2303.5 & 1228.5 & 50.0 \\
\hline (M) North of Room 52 & Basement & 2178.5 & 2288.5 & 50.0 \\
\hline (N) Rm203 & First Floor & -634.5 & 1132.5 & 492.0 \\
\hline (O) Lunch Room & First Floor & 541.5 & -847.5 & 492.0 \\
\hline (P) Room 327A & First Floor & 40.0 & 2332.5 & 492.0 \\
\hline (Q) Room 528 & First Floor & 2277.5 & 2340.5 & 492.0 \\
\hline (R) HLRF Truck Lock & First Floor & 3355.5 & 402.0 & 492.0 \\
\hline (S) Room 119 & First Floor & 2261.5 & 27.5 & 492.0 \\
\hline (T) Room 410 & First Floor & 1367.5 & 960.5 & 492.0 \\
\hline (U) Over Room 603 & Second & 44.5 & 2350.0 & 884.0 \\
\hline (V) Over Room 327 & Second & 2875.0 & 1520.0 & 1072.0 \\
\hline
\end{tabular}

\section{Subtask 8 - Modify the Calibration Procedure}

The energy bins used to characterize the spectrum striking the detectors were first set to the same energy bins as the Hansen-Roach 16 Group Cross-section library (Hansen, 1964). In practice this was far more energy bins than were needed for making adjustments to the calibration procedure. Eventually, it was found that only two energy groups were needed to do the analysis. It may be beneficial in some facilities to include more than two groups, but because there are limited options available for spectrum modification more energy groups may not provide much advantage. The techniques used 
for modifying the energy spectrum of neutrons and gammas were discussed in subtask 7 of Design Methodology. The two groups used for RPL were set to represent thermal and everything not thermal. The bin limits were zero to $4 \mathrm{eV}$ then from $4 \mathrm{eV}$ to $20 \mathrm{MeV}$.

There are only two viable options for altering the energy distribution of a neutron spectrum. Either thermalize them by scattering, or use Cadmium to remove thermal neutrons thereby increasing the ratio of fast neutrons. The bin structure was set to make utilizing these two methods easier and more effective. The Cadmium cutoff is around $0.5 \mathrm{eV}$ but there is a broader structure than just a single peak. Figure 11 shows the total neutron absorption cross-section for ${ }^{113} \mathrm{Cd}$. The peak is clearly visible but what is more important is the overall structure of the cross section.

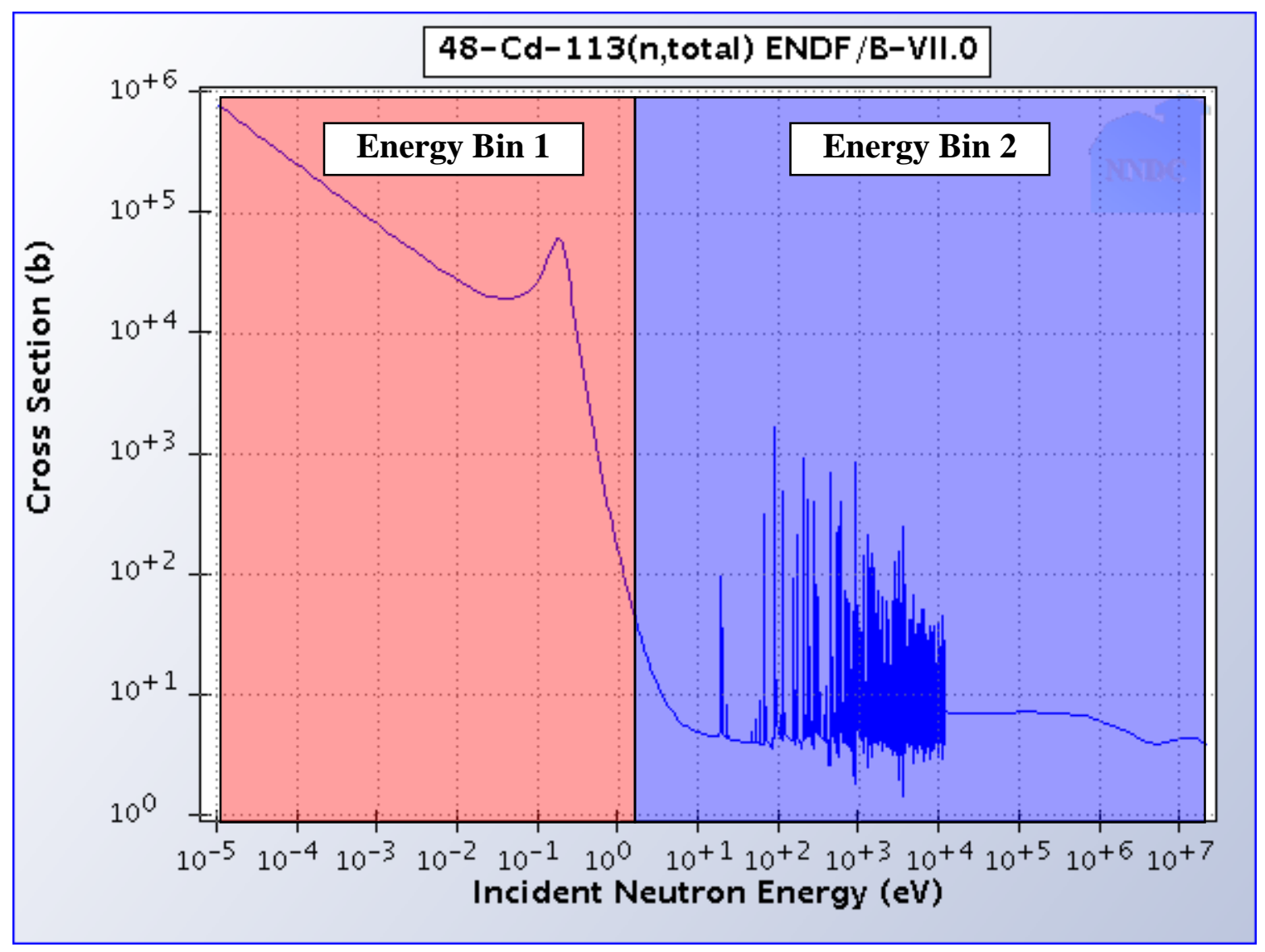

Figure 11. Graph of the total cross-section of ${ }^{113} \mathrm{Cd}$ 
The total cross-section is large (1000's of barns) up to the single digit $\mathrm{eV}$ range, which is the main driver for the bin structure used for the detector tallies.

It was found from computer simulation of the MAC that approximately two-thirds of the fluence striking the tally surfaces in the facility model was in the 0 to $4 \mathrm{eV}$ range with the remaining third above that. The opposite ratio was found for the existing Nuclear Criticality Detector calibration setup. The same tally in the calibration model showed that roughly one-third of the incident spectrum fell below $4 \mathrm{eV}$ and two-thirds above it. To prove the NCD were calibrated correctly, the calibration procedure had to be modified to better resemble the average spectrum of the MAC. The next part of the document goes into detail on how the analysis was performed and the results obtained.

\section{Example Nuclear Criticality Detector Calibration Procedure Evaluation}

The current calibration procedure for the NCD at PNNL utilizes a ${ }^{252} \mathrm{Cf}$ source that generates the neutron energy spectrum seen in Figure 12. 


\section{Cf-252 S.F. Watt Spectrum Coefficients}

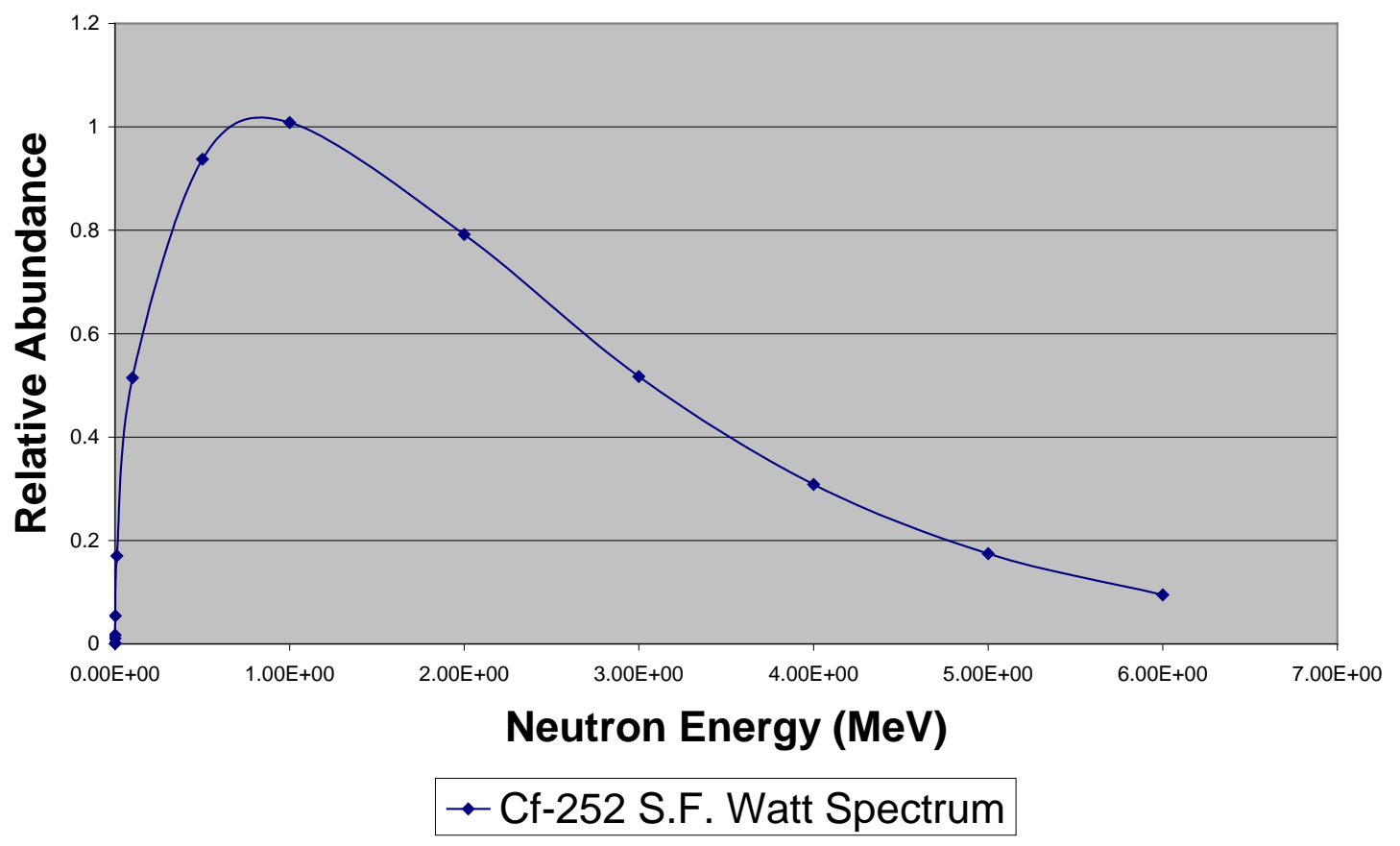

Figure 12. Neutron energy spectrum for the spontaneous fission of ${ }^{252} \mathrm{Cf}$

Placing the neutron emission spectrum of ${ }^{252} \mathrm{Cf}$ into the same 0 to $4 \mathrm{eV}$ and $4 \mathrm{eV}$ to 20MeV energy bins from the facility model yields the graph shown in Figure 13 -Note the graph is in Log Scale. 


\section{Energy Distribution of the Nuclear Criticality Detector Calibration Source}

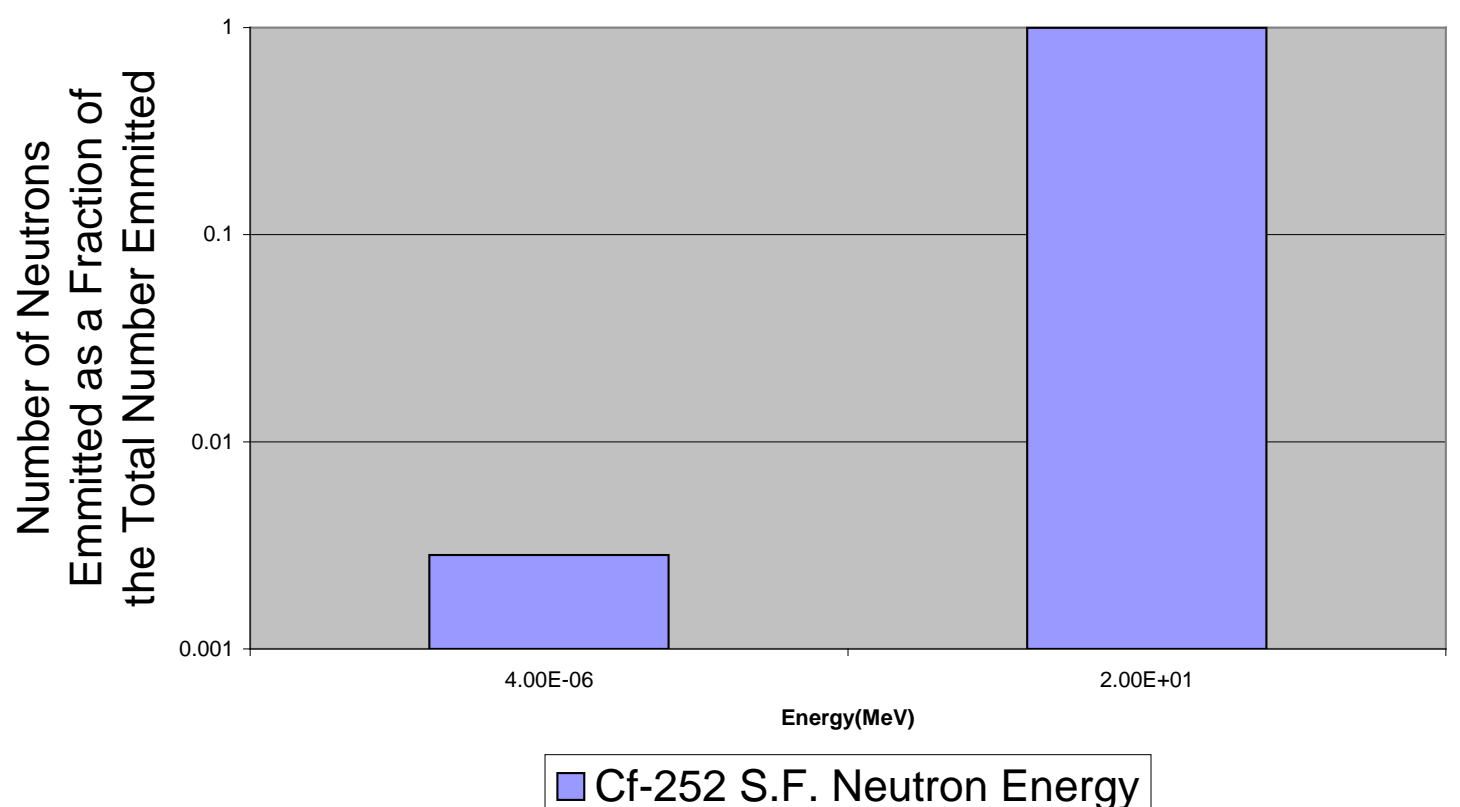

Figure 13. Neutron energy spectrum grouped into $4 \mathrm{eV}$ and $20 \mathrm{MeV}$ energy bins for the spontaneous fission of ${ }^{252} \mathrm{Cf}$ plotted on a log scale

It is clear from Figure 13 that the spontaneous fission of Cf-252 has almost no thermal energy neutrons.

The ${ }^{252}$ Cf source shown in Figure 12 and Figure 13 is placed on a motorized elevator in a 10 meter deep counting well. The NCD are placed on top of the well and the source is raised to the desired height for the corresponding activity level. For the current activity of the ${ }^{252} \mathrm{Cf}$ source, the distance between the elevator and the NCD is approximately 1.60 meters. The fluence that reaches the NCD was found to have the energy distribution shown in Figure 14. 


\section{Existing Calibration Spectrum}

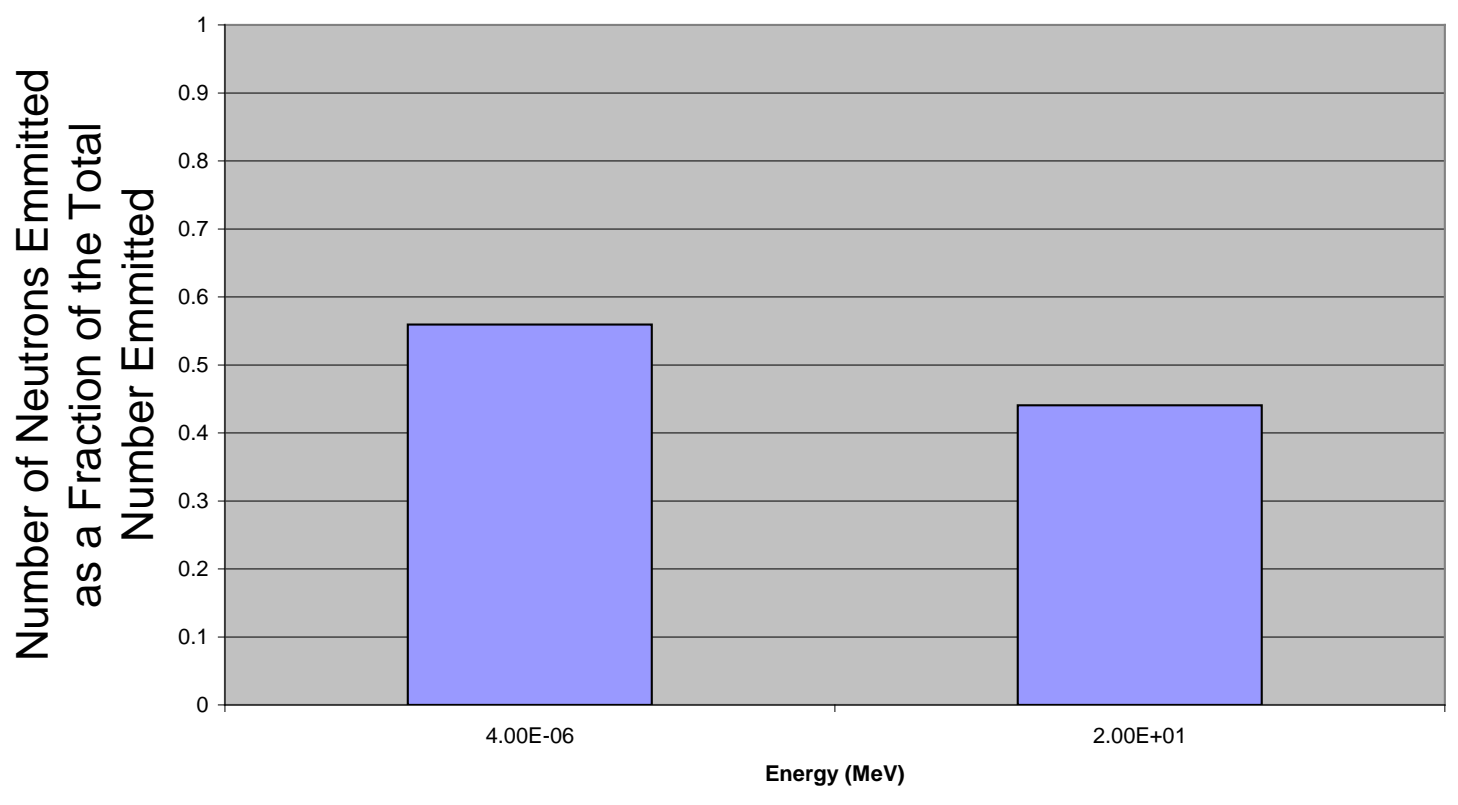

$\square$ Existing Calibration Spectrum

Figure 14. Existing calibration spectrum

Using the same energy bins as Figure 13 and Figure 14 for the MAC in RPL

generates the graph in Figure 15. It is clear that spontaneous fission spectrum of ${ }^{252} \mathrm{Cf}$ is considerably thermalized by the materials around the counting wells. 


\section{Neutron Energy Distribution Incident on the Nuclear Criticality Detector During the Minimum Accident of Concern}

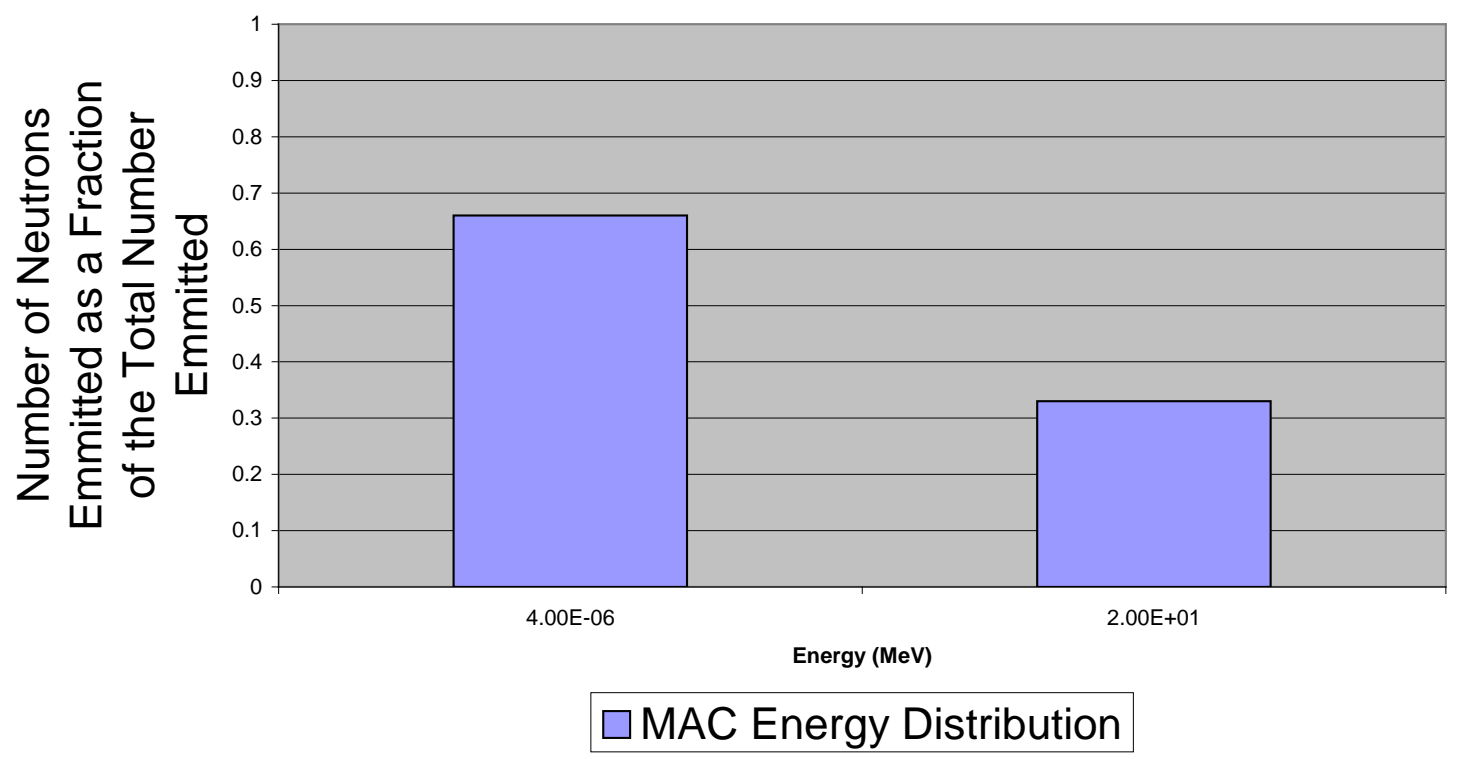

Figure 15. Average neutron energy spectrum during the Minimum Accident of Concern grouped into $4 \mathrm{eV}$ and $20 \mathrm{MeV}$ energy bins

From Figure 14 and Figure 15, it can be seen that the spectrum used to calibrate the NCD was not the same spectrum the NCD was likely to be exposed to during the MAC; therefore it was necessary to modify how the NCD were calibrated. The existing calibration spectrum had approximately 56 percent of the spectrum below $4 \mathrm{eV}$ whereas the MAC spectrum had 66 percent. This discrepancy had to be resolved because if there was spectral dependence for the counting efficiency then the assumed known count rates used in subtask 4 would be incorrect.

To perform the analysis, an MCNP5 model was constructed. The basics of the calibration setup were included and flux tallies were used to find the energy distribution of neutrons striking the detector. Different materials were placed between the source and 
the detector until the required energy distribution was obtained. A simple diagram of the calibration setup is shown in Figure 16. The NCD is yellow, the counting well is gray, and the ${ }^{252} \mathrm{Cf}$ source holder is red. The drawing is to scale.

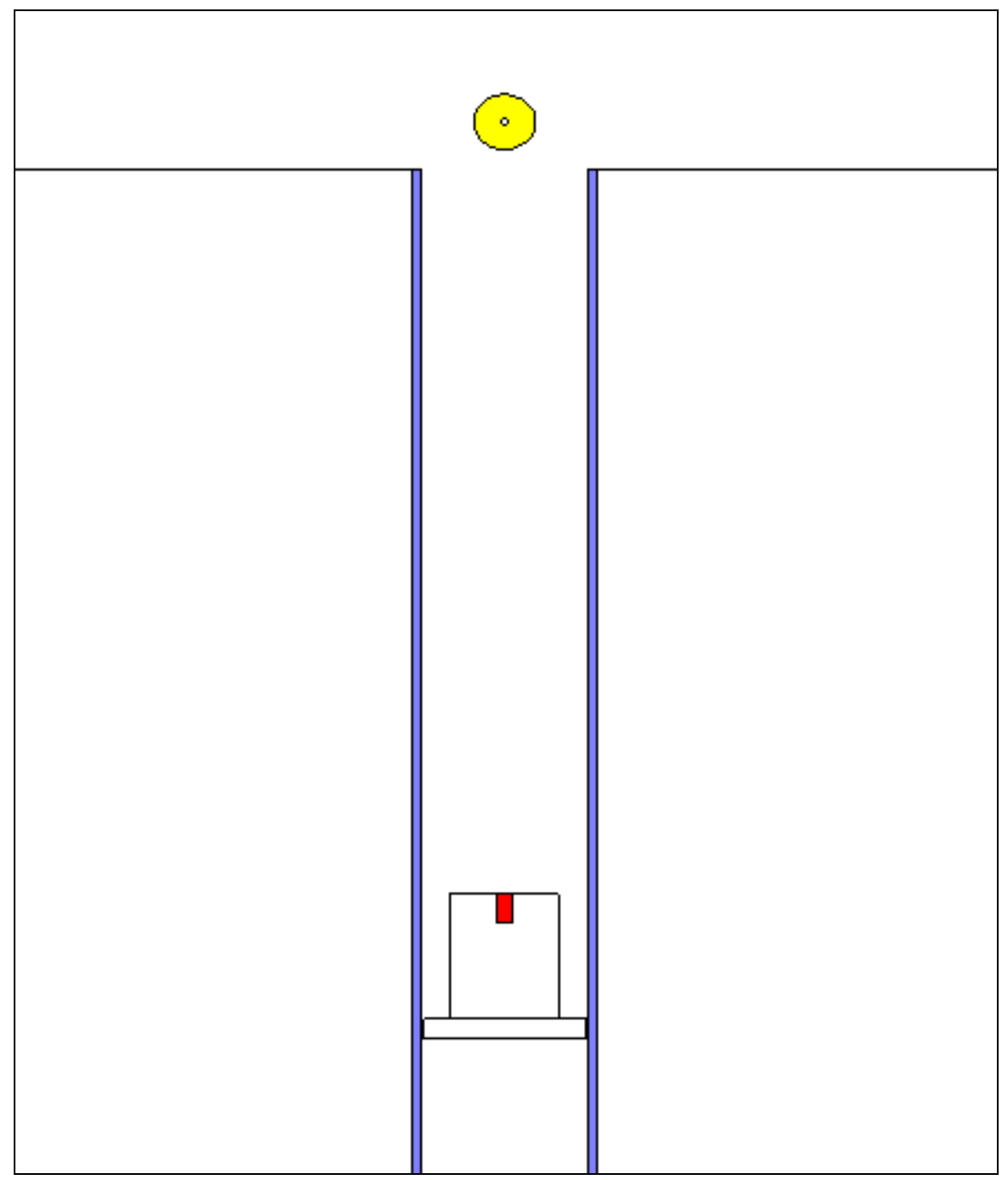

Figure 16. Existing NCD calibration setup

The entire well is surrounded by high density concrete. It was found that the following two steps could be inserted into the existing calibration spectrum to more effectively bound the average energy spectrum present during the MAC: 
1. Construction and Placement of a $2 \mathrm{~cm}$ thick, $13 \mathrm{~cm}$ radius disc of High Density Polyurethane (HDPE) between the Californium Source and the NCD. The location of the plate should be just below the NCD.

2. Construction and placement of a hollow five sided box of HDPE $40 \mathrm{~cm} \times 40 \mathrm{~cm} x$ $24 \mathrm{~cm}$ around the NCD on top of the counting well. All sides of the box being roughly $6 \mathrm{~cm}$ thick.

Figure 17 shows what the revised calibration setup will look like. 


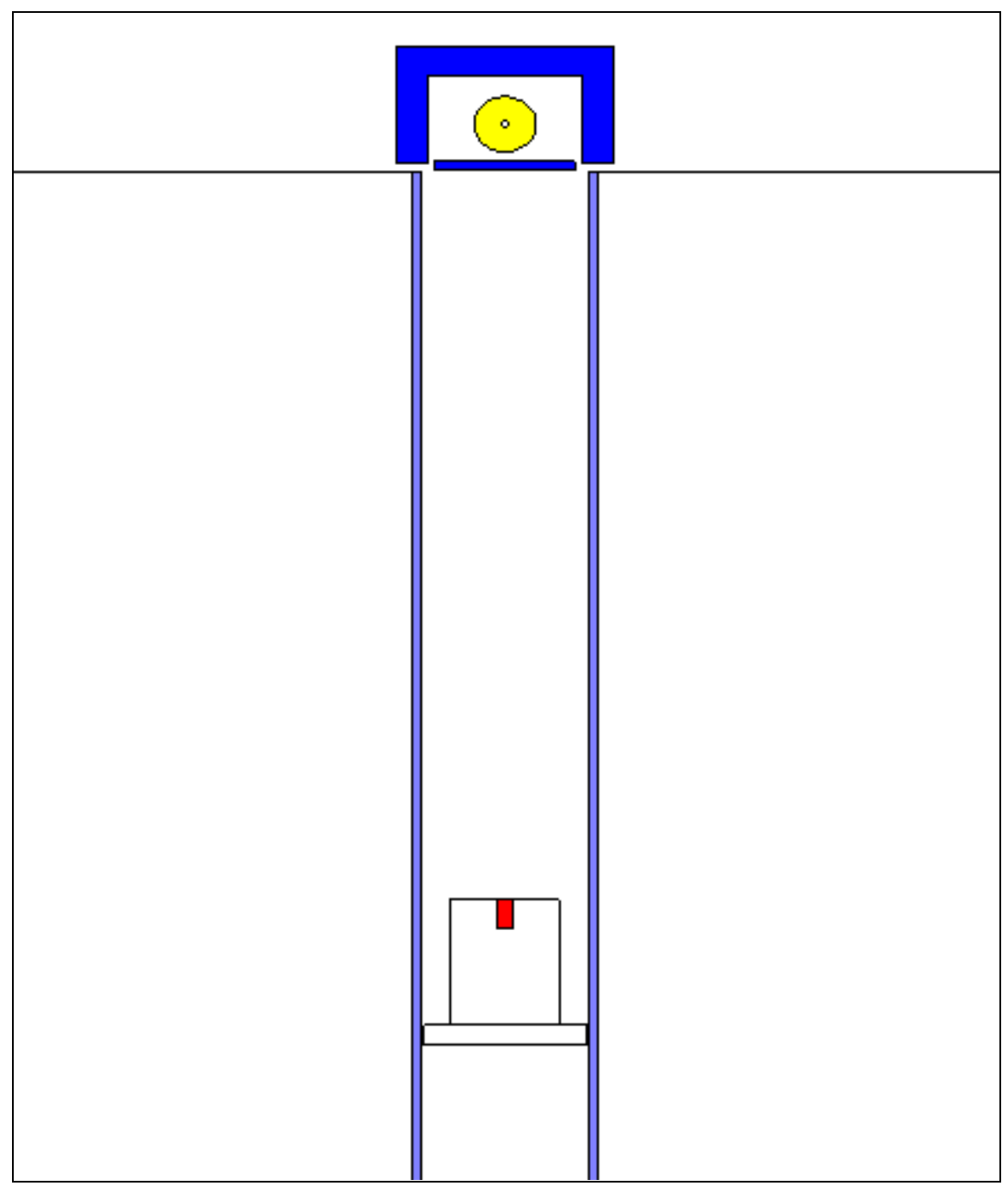

Figure 17. Revised NCD calibration setup with the added HDPE

The blue material is the added HDPE. After the proposed HDPE pieces are implemented, the spectrum hitting the NCD is altered to the distribution shown in Figure 18. 


\section{Calibration Spectrum After Proposed Changes}

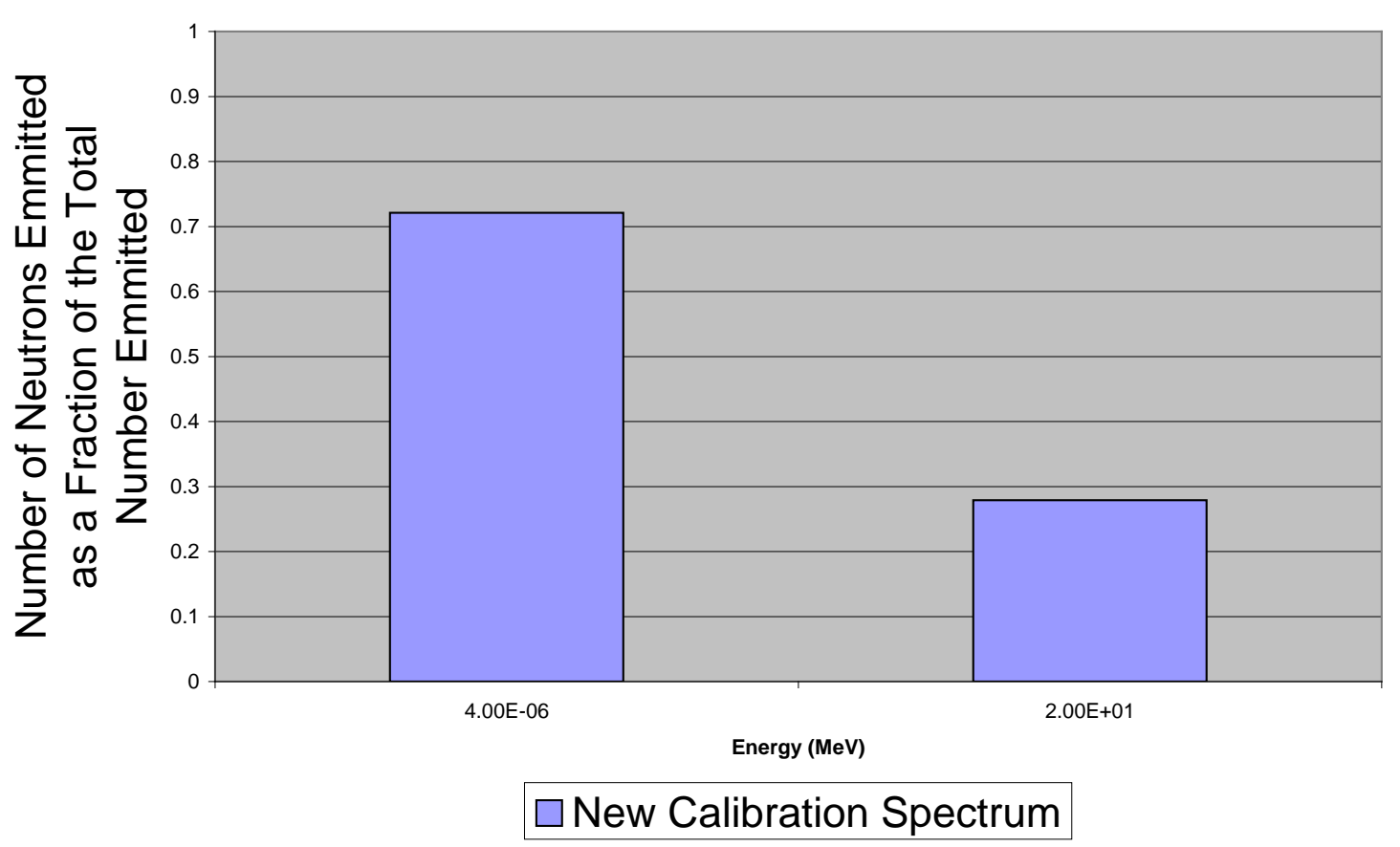

Figure 18. Modified calibration spectrum grouped into $4 \mathrm{eV}$ and $20 \mathrm{MeV}$ energy bins

Adding the HDPE to the calibration process creates a spectrum with a slightly larger thermal fraction than both the existing calibration setup and the MAC spectrum. In reality, there is a range of spectrums that are possible in the MAC. Therefore the increase in the thermal fraction to beyond the MAC is done intentionally to more effectively bound the calibration of the NCD. The HDPE slab used to slow down the neutrons also absorbed or reflected away a considerable number of them as well. The box was placed over the well to increase the amount of reflection back toward the NCD. Placing the box in this manner not only made up for what was lost to the HDPE slab but actually increased the fluence striking the detector beyond the existing calibration procedure by an extra 15 percent. Californium neutron sources are very expensive so prolonging their 
usable life is very helpful. Table 7 gives a summary of all the values shown in the histograms.

Table 7. Corresponding Data Used in the Histograms

\begin{tabular}{|c|c|c|c|c|}
\hline $\begin{array}{c}\text { Energy } \\
\text { Bins } \\
(\mathbf{M e V})\end{array}$ & $\begin{array}{c}\text { Cf-252 } \\
\text { Emission } \\
\text { Spectrum }\end{array}$ & $\begin{array}{c}\text { MAC } \\
\text { Average } \\
\text { Spectrum }\end{array}$ & $\begin{array}{c}\text { Old } \\
\text { Calibration } \\
\text { Spectrum }\end{array}$ & $\begin{array}{c}\text { New } \\
\text { Calibration } \\
\text { Spectrum }\end{array}$ \\
\hline $4.00 \mathrm{E}-06$ & 0.003 & 0.660 & 0.559 & 0.721 \\
\hline $2.00 \mathrm{E}+01$ & 0.997 & 0.330 & 0.441 & 0.279 \\
\hline
\end{tabular}

The suggested changes discussed here only deal with the need to modify the calibration spectrum to better mimic the likely accident conditions. These proposed changes are intended as an addition to the existing calibration procedure, not as a total replacement of existing methods.

\section{Subtask 9 - Apply Variance Reduction as needed}

During the modeling portion of the project it was found that the run times needed to obtain usable precision (less than 0.10 error) were very large. For the majority of the MAC locations needed to show complete facility coverage, the run times were upwards of 10 days on dual quad core 3.2GHz workstations. With 24 accident locations selected to prove coverage, it is apparent that if all went correctly it would take a minimum of 240 days to receive results. This amount of time exceeded the schedule of the project. The decision was then made to investigate using variance reduction in MCNP5.

The QA program at PNNL very clearly states that no variance reduction techniques may be used that alter the fundamental physics of the computer simulation. It was attempted to implement reduction strategies that complied with this requirement but 
no solution was found that reduced run times to an acceptable level. To mitigate this problem, a compromise was made. Well documented variance reduction techniques could be used provided that standard Monte Carlo runs would be completed in the future to validate the results.

After extensive investigation, it was found through trial and error that implementing the deterministic transport option in MCNP5, DXTRAN, reduced the run times to 4 days plus or minus 2 days depending on the accident location. To initially verify the DXTRAN results were correct, 2 standard Monte Carlo runs were carried out. The first standard run represented the hardest accident location to detect, Room 90, a well-shielded location at the northern edge of the basement. The second run was for the easiest location to detect in the facility, (T) Room 410, a laboratory located in the center of the first floor. In both cases there was very little deviation of the DXTRAN results from standard Monte Carlo. Table 8 shows the results for these two sets of runs.

Table 8. Comparison of deterministic transport results to standard Monte Carlo results

\begin{tabular}{|l|c|c|c|c|c|}
\hline \multicolumn{1}{|c|}{$\begin{array}{c}\text { Accident } \\
\text { Location }\end{array}$} & $\begin{array}{c}\text { Detector } \\
\mathbf{2}\end{array}$ & $\begin{array}{c}\text { Detector } \\
\mathbf{3}\end{array}$ & $\begin{array}{c}\text { Detector } \\
\mathbf{4}\end{array}$ & $\begin{array}{c}\text { Detector } \\
\mathbf{5}\end{array}$ & $\begin{array}{c}\text { Detector } \\
\mathbf{6}\end{array}$ \\
\hline $\begin{array}{l}\text { (T) Room 410 - } \\
\text { Standard }\end{array}$ & $1.18 \mathrm{E}+09$ & $1.18 \mathrm{E}+08$ & $1.79 \mathrm{E}+07$ & $1.34 \mathrm{E}+07$ & \\
\hline $\begin{array}{l}\text { (T) Room 410 - } \\
\text { DXTRAN }\end{array}$ & $1.18 \mathrm{E}+09$ & $1.17 \mathrm{E}+08$ & $1.73 \mathrm{E}+07$ & $1.25 \mathrm{E}+07$ & \\
\hline $\begin{array}{l}\text { 100*(Standard } \\
\text { - DXTRAN)/ } \\
\text { Standard }\end{array}$ & -0.1 & 0.7 & 3.2 & 6.8 & \\
\hline $\begin{array}{l}\text { (K) Room 90 - } \\
\text { Standard }\end{array}$ & $5.57 \mathrm{E}+05$ & $2.03 \mathrm{E}+05$ & $2.34 \mathrm{E}+05$ & & $1.97 \mathrm{E}+05$ \\
\hline $\begin{array}{l}\text { (K) Room 90 - } \\
\text { DXTRAN }\end{array}$ & $5.58 \mathrm{E}+05$ & $2.02 \mathrm{E}+05$ & $2.29 \mathrm{E}+05$ & & $1.96 \mathrm{E}+05$ \\
\hline $\begin{array}{l}\text { 100*(Standard } \\
\text { - DXTRAN)/ } \\
\text { Standard }\end{array}$ & -0.1 & 0.2 & 2.2 & & 0.5 \\
\hline
\end{tabular}


As mentioned earlier, extreme caution must be used when applying these techniques. Even though promising results have been demonstrated for this technique, the final analysis will not accept them. As per QA requirements, the final results will not be established on record until the standard Monte Carlo runs are complete. Techniques that reduce the variance have tremendous use for scoping and preliminary analysis, but are generally not acceptable as final numbers for the project.

\section{Subtask 10 - Perform sensitivity analysis on the final CAS design}

The sensitivity analysis conducted for RPL focused primarily on the effects of overloading the spaces in the facility with material that was twice as dense as the Full Office and Full Laboratory material definitions listed in Appendix C. This fill density was chosen because it represents the uppermost limit of the physical capacity of the spaces. Also the distribution of the super dense spaces was configured in the most challenging manner for accident detection. The spaces in the core of the building were filled with the super dense material and the spaces outside of the accident locations were left empty. Arranging the fill material in this manner represented a situation where the maximum amount of matter is placed between accidents and detectors. This represents

the most limiting feasible scenario for the sensitivity analysis. Results for (T) Room 410 and for (K) Room 90 are given in Table 9. 
Table 9. Detector counts for preliminary sensitivity analysis for maximum loading within the facility

\begin{tabular}{|r|c|c|c|c|c|c|}
\hline $\begin{array}{c}\text { Accident } \\
\text { Location }\end{array}$ & NCD 2 & NCD 3 & NCD 4 & NCD 5 & NCD 6 & $\begin{array}{c}\text { Number } \\
\text { Alarming }\end{array}$ \\
\hline Room 410 & $1.18 \mathrm{E}+09$ & $1.17 \mathrm{E}+08$ & $1.73 \mathrm{E}+07$ & $1.25 \mathrm{E}+07$ & & $\mathbf{4}$ \\
\hline $\begin{array}{r}\text { Overloaded } \\
\text { Tally }\end{array}$ & $5.83 \mathrm{E}+08$ & $5.20 \mathrm{E}+07$ & $2.10 \mathrm{E}+06$ & $1.39 \mathrm{E}+06$ & & $\mathbf{4}$ \\
\hline $\begin{array}{r}\text { \% Lost } \\
\text { from }\end{array}$ & 50 & 56 & 88 & 89 & & \\
Overloading & $5.58 \mathrm{E}+05$ & $2.02 \mathrm{E}+05$ & $2.29 \mathrm{E}+05$ & & $1.96 \mathrm{E}+05$ & $\mathbf{4}$ \\
\hline Room 90 & $3.71 \mathrm{E}+05$ & $1.16 \mathrm{E}+05$ & $1.19 \mathrm{E}+05$ & & $9.67 \mathrm{E}+04$ & $\mathbf{4}$ \\
\hline $\begin{array}{r}\text { Overloaded } \\
\text { Tally }\end{array}$ & 43 & 48 & & 51 & \\
\hline $\begin{array}{r}\text { \% Lost } \\
\text { from } \\
\text { Overloading }\end{array}$ & 34 & & & & \\
\hline
\end{tabular}

Again these results were obtained using deterministic transport and are not acceptable in the final report, but these preliminary results indicate that four detector coverage is maintained even at this loading. The standard Monte Carlo runs will provide the final data when they are completed sometime in the 2015 timeframe. It should be noted for the overloaded models, both standard and deterministic based MCNP5 run times increased dramatically.

\section{Show the calibration method follows simple and concise methodology for setting the minimum detector trip points;}

Simplifying the calibration method for setting the minimum detector trip points was fairly straightforward. The majority of the analysis needed to set the trip setting was completed after both the average MAC spectrum in the facility had been analyzed and the calibration setup modeled for subtask 4. Because of the methodology used to establish building coverage took into account the conversion from simulated count rate to actual count rate, all that remained was to state what that count rate was. 
Given the historical absence of false alarms with the established trip setting for RPL, the decision was made to attempt establishing coverage for the entire building at or above that level. It was shown in Chapter 4 subtask 7 that coverage was established for the entire facility above the historical trip setting. The final revision of the calibration procedure simply removed all extraneous dose conversion wording to arrive at the following definition of the trip setting: "All Nuclear Criticality Detectors for the Radioisotope Production facility will be set to alarm at 563 counts per second.” This is the definition that will be submitted to the DOE to show compliance with their request for a more transparent and verifiable definition of the minimum detector trip setting.

\section{Assemble the final report;}

The final design report for the CAS of RPL was drafted in two parts that roughly followed the outline listed under the corresponding heading in Design Methodology. The initial draft contained four sections which were: the Introduction, Design Description, Requirements Documentation, and Methodology. The second draft contained:

- Evaluation and Results

- Conclusions

- References

Each draft was reviewed by a committee, and their comments were all submitted for evaluation of relevance. Because an outline was already provided, only general comments about the specific RPL report will be addressed here.

There is a reason the heading for this section is titled "Assemble the Final Report," not "Write the Final Report." In reality there is little writing that goes into the 
final design report. What actually goes into the report is primarily tables, figures, diagrams, and references. It is advised to make all graphs, charts, etc., as plain and uncluttered as possible. Nothing seems to annoy management and tech editors more than a busy graphic.

The most challenging aspect of assembling the final report was finding, organizing, and citing all of the required References. The final design report contained over 60 different references, and hard copies had to be obtained for all of them. PNNL procedure required the references to be in the same order in the appendix that they were presented in the body of the text. PNNL document control system is also very strict, which made obtaining the necessary hard copies time consuming. It can not be overstated how important it is to retain all references that are encountered throughout the design phase, regardless of their perceived importance at that time. 


\section{Chapter 5 - Conclusion}

\section{Summary}

A guide for designing a Criticality Alarm System was established. The focus of the guide was on producing a CAS that would meet applicable regulations and supply dependable coverage for personnel. The first half of the document provided an outline for the methodology behind each task and then thoroughly evaluated each task from a technical perspective. The second half of the document demonstrated how to apply this methodology by reviewing the analysis performed on the CAS of the Radiological Processing Laboratory of Richland, Washington. Results from the RPL system were included for each task and subtask. Potential pitfalls that were encountered during the design process were also included to aid the user in avoiding costly mistakes.

\section{Future Work}

As technology and standards evolve, the requirements that will be placed on Criticality Alarm Systems will no doubt change, likely only becoming more stringent. To meet any increased constraints, it is likely that more labor and computational time will be needed to complete the design of a CAS. As computational technology advances, more in-depth and detailed analyses can be conducted that could utilize greater precision. Greater modeling detail will instill greater confidence in the results and reliance on established overly conservative methods could potentially be relaxed. 
The development of more efficient detectors, as well as more in-depth analysis, would allow the minimum trip settings to be raised thereby increasing the margin for avoiding false alarms.

\section{Enhancements}

Several improvements were made to the existing CAS design for RPL:

- Nearly all of the RPL facility was modeled and evaluated

- The number of modeling assumptions used was decreased

- Complete facility detector coverage was proven

- Detector coverage for the MAC was increased from 2 detectors to 4

- Better calibration methods were developed that more effectively bound the average spectrum likely to strike the NCD during the MAC

- The definition of the minimum trip setting was vastly streamlined to meet regulatory findings and to enhance transparency

- Direct dose calculation for the MAC was done, thereby eliminating the need for fluence to dose conversion factors

Each of these improvements increases the reliability of the CAS and streamlines any potential future analysis that may need to be performed. The increased detector coverage opens more areas in the facility where criticality work can be conducted. The increase in the number of detectors that will see the MAC impacts the operations in the building by allowing criticality work to continue even in the event of two separate detector failures. The modification of the calibration procedure enhances the reliability of the CAS by demonstrating that the NCD will more effectively see the energy spectrum of neutrons emitted during the MAC. Changing the definition of the minimum trip setting from a dose value to a simple count rate makes understanding what the CAS is set 
to alarm for much easier. Changing the trip setting to a count rate also satisfies regulatory findings. The elimination of the dose conversion factors makes finding the corresponding fluence of the MAC clearer, again enhancing transparency of the overall design methodology.

The combination of all these improvements to the CAS in RPL provides better, more dependable coverage, which will send out prompt warning to personnel in the event of a criticality potentially saving lives of those involved. 


\section{Definitions}

Criticality Alarm System (CAS): The combination of nuclear criticality detectors, audible alarms, and comparator panel that is responsible for the prompt detection and immediate audible alarm issuance to personnel.

Detector Cell Tally Efficiency (DCTE): The ratio of the number of particles that strike the detector cell equivalent being tallied that will ultimately result in a count, this is a computational quantity.

Detector Efficiency: The ratio of the number of particles entering the active detector volume which result in a count, which is experimentally determined.

Detector Surface Tally Efficiency (DSTE): The ratio of the number of particles that strike the tally surface that will ultimately result in a count, this is a computational quantity.

High Density Polyurethane (HDPE): A polymer with the chemical composition of CH2 with an assumed density of .933(g/cc).

High Level Radiation Facility (HLRF): An annex of building 325 in the 300 area of the Hanford, Washington Department of Energy site that contains large shielded hot cells for work with fissile material. 
Monte Carlo N-Particle Transport Code Version 5 (MCNP5): A Monte Carlo based neutronics code developed by Los Alamos National Laboratories and maintained by the Radiation Safety Information Computational Center.

Nuclear Criticality Detector (NCD): The instrument used for the detection of particles resultant from fission.

Radioisotope Production Laboratory (RPL): Designated as building 325 in the 300 area of the Hanford, Washington Department of Energy site used for work conducted on fissile material, a Class 2 hazard facility.

Shielded Analytical Laboratory (SAL): An annex of building 325 in the 300 area of the Hanford, Washington Department of Energy site that contains shielded hot cells for work with fissile material. 


\section{Appendix A: RPL Building Schematics with Accident and Detector Locations}

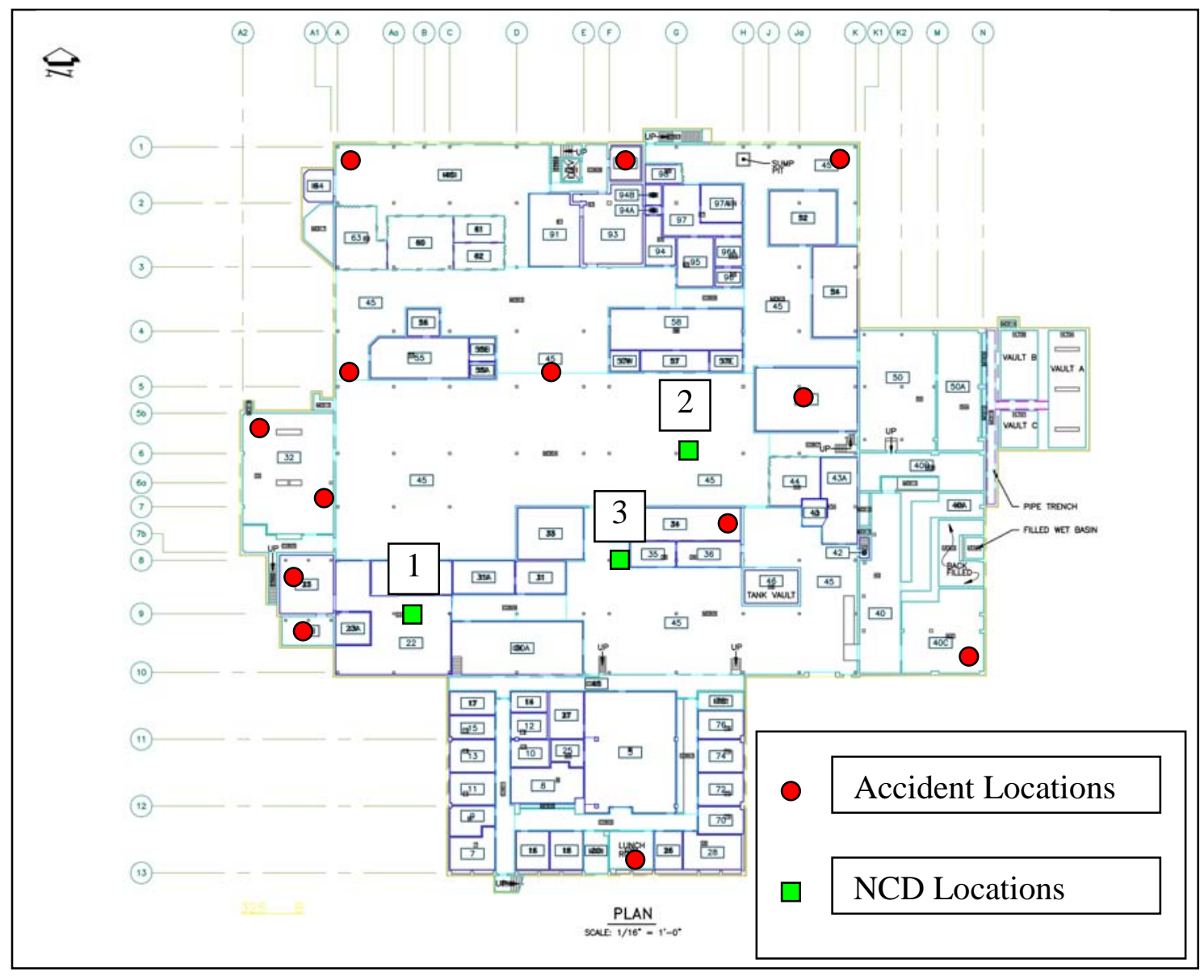

Figure 19. RPL Basement showing accident locations and NCD locations 


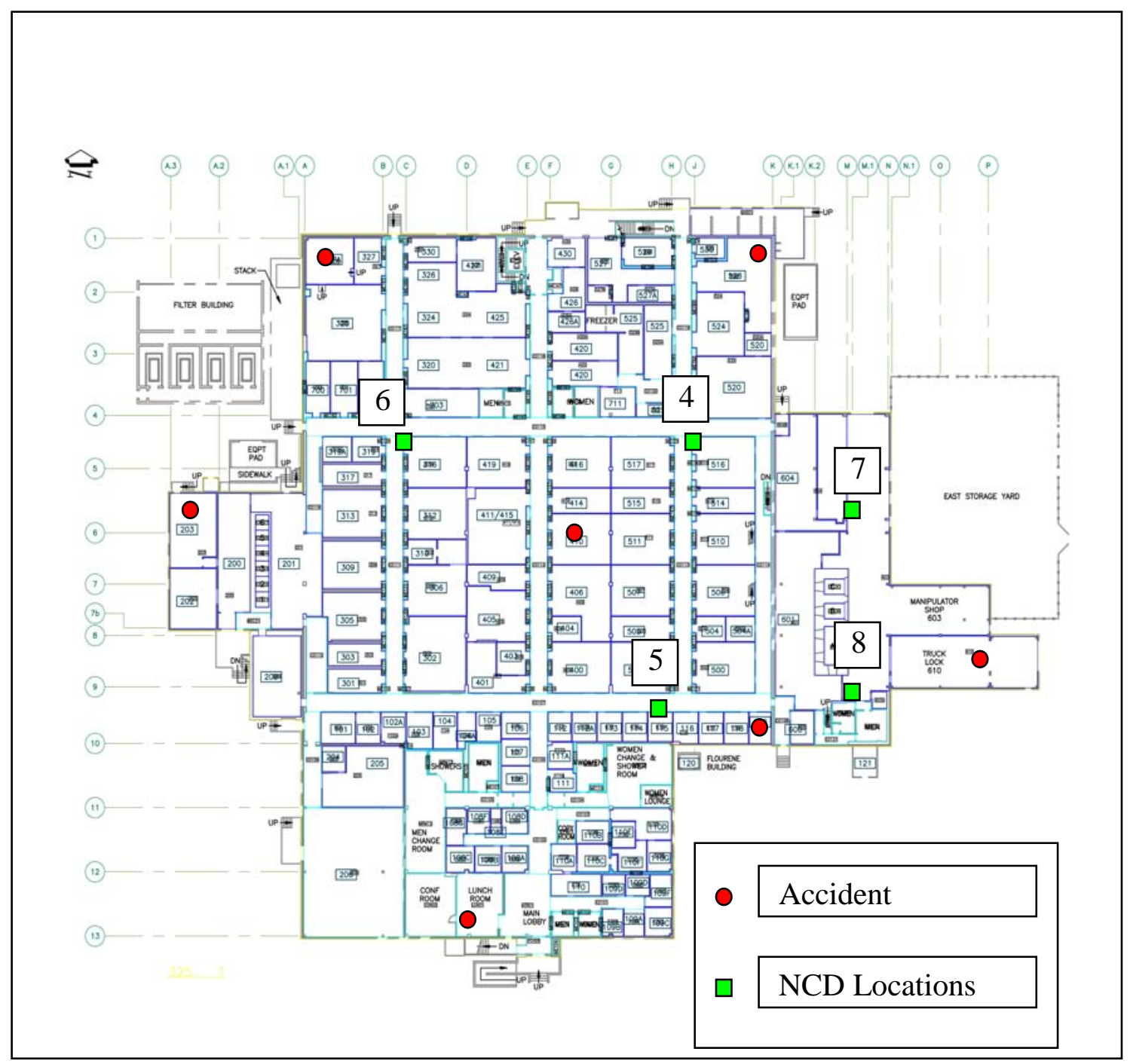

Figure 20. RPL First Floor showing accident locations and NCD locations 


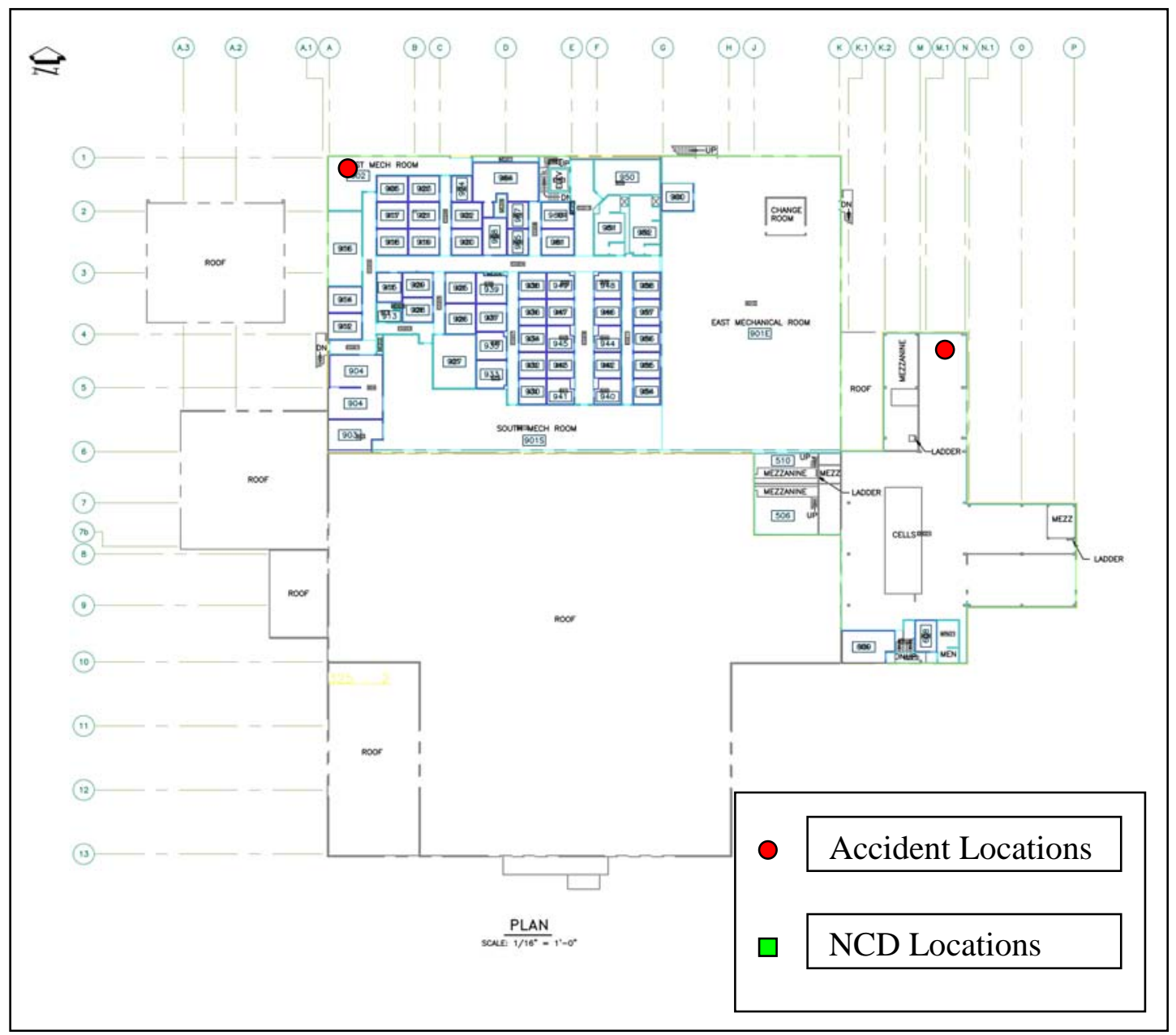

Figure 21. RPL Second Floor showing accident locations, no NCD are located on this floor 


\section{Appendix B: CAS Component Descriptions}

\section{Technical Description of the Comparator Panel}

ANSI/ANS-8.3-1997 dictates that the comparator panel shall not trigger a criticality alarm for any single malfunctioning detector and that great care must be taken in the avoidance of false alarms. The panel constantly generates a signal that is broadcast to all of the detectors in the system. If any detector ceases to return the signal, the board considers the detector failed, and a warning is generated. Note that this warning is not the audible criticality alarm; it is a different alarm that is sent to an area normally occupied by competent personnel. Another note about the panel is that it does not issue a criticality alarm until two or more channels have alarmed. This eliminates the possibility of a single detector failure causing a false alarm. This is an important design criterion that greatly impacts the physical design of the system. It should also be noted the ANSI/ANS-8.3-1997 words the alarm statement as “...requiring concurrent response of two or more detectors to initiate the alarm.” So it is allowable to require greater than two concurrent detector responses for a criticality alarm to be generated.

\section{Technical Description of the Radiation Detectors}

There are two types of radiation resultant from fission that are practically detectable: neutrons and gamma rays. The range of both neutrons and gammas is large enough that the number of detectors needed for adequate coverage is not prohibitive. 
Typical CAS use either neutron or gamma detectors. A review of the literature did not identify any system that uses a combination of the two.

The selection of neutron or gamma ray detectors for the detection system has some important physical implications. Because the goal of the alarm system is to establish adequate coverage for the facility in question, it is desired from an engineering standpoint to set the trip setting of the detectors as low as possible. The lower the trip setting is then the fewer particles the detectors need to see for an alarm signal to be generated. But conversely the lower the trip setting is, the greater the risk of false alarm becomes. So there must be a balance made between lowering the minimum trip setting for detection limits and keeping the trip setting high enough to avoid false alarms. Finding and proving this balance is not trivial so appropriate detector selection is crucial. Section 5.1 of ANSI/ANS-8.3-1997 addresses the reliability of the system in general, which is of course applicable to the detectors themselves. It says:

"The system shall be designed for high reliability and should utilize components that do not require frequent servicing, such as lubrication or cleaning. The system should be designed to minimize the effects of nonuse, deterioration, power surges, and other adverse conditions. The design of the system should be as simple as is consistent with the objectives of ensuring reliable actuation of the criticality alarm signal and avoidance of false alarms."

This description is used to narrow the field of possible detectors that could be used in the system.

To investigate detector specific properties the book, Radiation Detection and Measurement by Glenn Knoll is extraordinarily useful. It goes into great detail for many different detector types. It provides operational data as well as counting efficiencies. It should be clear that the main detector selection criteria are gross count efficiency, 
dependability, and the detection efficiency over the applicable incident particle energy range i.e., fission. There is no need for a detector that has spectral analysis capability. The list of potential detector candidates for both gamma and neutron detectors is quite large and beyond the focus of this document. What is of concern is the selection of which type of particle will be detected.

Background levels, both terrestrial and cosmic, are considerably different for gammas and neutrons. Background levels for neutrons are typically next to nothing in most areas. Generally it takes human effort to liberate neutrons, so man made sources are really the only area of concern for false alarms in a system utilizing neutron detection. This is not the case for gamma rays.

Natural background for gamma rays varies from location to location, sometimes considerably. Worse still is that the background levels for gamma rays can fluctuate with time. For example cosmic sources, such as solar flares, can cause significant increases in the gamma ray background levels. It is not all bad though because gamma rays are not attenuated in low $\mathrm{Z}$ materials as quickly as neutrons are. Most building construction materials are low-Z; concrete, wood, sheet rock, to name a few. Of course there can be large amounts of metal in buildings as well. Metals do a reasonably good job of shielding gammas. But metal will also absorb neutrons, which will then emit gammas as a result. This makes typical activity levels of gammas in facilities higher than that of neutrons during accident scenarios and therefore easier to detect.

Because the 300 area of Hanford, Washington is undergoing widespread demolition, the radiation detectors from other buildings have been salvaged and stockpiled. The majority of these detectors are $\mathrm{BF}_{3}$ filled neutron proportional detectors. 
These are very reliable, simple devices that don’t require any significant maintenance other than calibration. So it was "recommended" that the design utilize this excess resource, which it does.

Equation B-1 shows the detection efficiency of $\mathrm{BF}_{3}$ Tube. $\sum_{\mathrm{a}}(\mathrm{E})$ is the Macroscopic Absorption Cross-Section of $\mathrm{B}^{10}$ at an energy level $\mathrm{E}$, and $\mathrm{L}$ is the active length of the tube. Be sure to note any end effects when making an active length determination.

$$
\mathrm{E}(\mathrm{E})=1-\exp \left[-\sum_{\mathrm{a}}(\mathrm{E}) * \mathrm{~L}\right]
$$

This equation was provided to illustrate a point. The absorption cross-section of $\mathrm{B}^{10}$ is very large so the corresponding detection efficiency is good. 


\section{Appendix C: Material Definitions for Codes}

\begin{tabular}{|c|c|}
\hline \multicolumn{2}{|c|}{$32 \mathrm{~g} / \mathrm{l}^{239} \mathrm{Pu}$ in $\mathrm{H}_{2} \mathrm{O}$ (From ARH 600) } \\
\hline Nuclide & Number Density (atoms/bn-cm) \\
${ }^{239} \mathrm{Pu}$ & $8.0614 \mathrm{E}-05$ \\
${ }^{16} \mathrm{O}$ & $3.3374 \mathrm{E}-02$ \\
${ }^{1} \mathrm{H}$ & $6.6748 \mathrm{E}-02$ \\
\hline
\end{tabular}

\begin{tabular}{|c|c|}
\hline \multicolumn{2}{|c|}{$\mathrm{H}_{2} \mathrm{O}$} \\
\hline Nuclide & Number Density (atoms/bn-cm) \\
${ }^{1} \mathrm{H}$ & $6.6890 \mathrm{E}-02$ \\
${ }^{16} \mathrm{O}$ & $3.3440 \mathrm{E}-02$ \\
\hline
\end{tabular}

\begin{tabular}{|c|c|}
\hline \multicolumn{2}{|c|}{ Iron } \\
\hline Nuclide & Mass Density $\left(\mathrm{g} / \mathrm{cm}^{3}\right)$ \\
$\mathrm{Fe}$ & 7.874 \\
\hline
\end{tabular}

\begin{tabular}{|c|c|}
\hline \multicolumn{2}{|c|}{ Air } \\
\hline $\begin{array}{c}\text { Nuclide } \\
{ }^{14} \mathrm{~N}\end{array}$ & Weight Fraction \\
$79 \%$ \\
${ }^{16} \mathrm{O}$ & $21 \%$ \\
\multicolumn{2}{|c|}{ Atom Density=1.197E-03 atoms/bn-cm } \\
\hline
\end{tabular}

\begin{tabular}{|c|c|}
\hline \multicolumn{2}{|c|}{ Dirt } \\
\hline Nuclide & Atom Fraction \\
Si (nat.) & $33 \%$ \\
${ }^{16} \mathrm{O}$ & $67 \%$ \\
Mass Density $=2.32 \mathrm{~g} / \mathrm{cm}^{3}$. With a $66 \%$ packing factor mass density $=1.53 \mathrm{~g} / \mathrm{cm}^{3}$. \\
\hline
\end{tabular}




\begin{tabular}{|c|c|}
\hline \multicolumn{2}{|c|}{ Concrete (2.3g/cc) } \\
\hline Nuclide & Atom Fraction \\
${ }^{27} \mathrm{Al}$ & 0.00175 \\
$\mathrm{Ca}$ (nat.) & 0.00152 \\
$\mathrm{Fe}$ (nat.) & 0.00035 \\
${ }^{1} \mathrm{H}$ & 0.01375 \\
${ }^{16} \mathrm{O}$ & 0.04608 \\
${ }^{23} \mathrm{Na}$ & 0.00175 \\
$\mathrm{Si}$ (nat.) & 0.01663 \\
\hline
\end{tabular}

\begin{tabular}{|l|c|}
\hline \multicolumn{2}{|c|}{ Stainless Steel } \\
\hline Nuclide & Weight Fraction \\
Fe (nat.) & $74 \%$ \\
Cr (nat.) & $18 \%$ \\
Ni (nat.) & $8 \%$ \\
\hline
\end{tabular}

\begin{tabular}{|c|c|}
\hline \multicolumn{2}{|c|}{ Stainless Steel Walls } \\
\hline Nuclide & Atom Density (atoms/bn-cm) \\
Fe (nat.) & $2.63792 \mathrm{E}-03$ \\
Cr (nat.) & $6.89167 \mathrm{E}-04$ \\
Ni (nat.) & $2.71250 \mathrm{E}-04$ \\
Total & $3.59833 \mathrm{E}-03$ \\
\hline
\end{tabular}

Wall density changes from the 8" to the 4" because of construction method

\begin{tabular}{|c|c|}
\hline \multicolumn{2}{|c|}{ 8” Wood Walls } \\
\hline Nuclide & Mass Density $\left(\mathrm{g} / \mathrm{cm}^{3}\right)$ \\
C (nat.) & $2.6664 \mathrm{E}-02$ \\
${ }^{1} \mathrm{H}$ & $3.7320 \mathrm{E}-03$ \\
${ }^{16} \mathrm{O}$ & $2.9604 \mathrm{E}-02$ \\
\hline
\end{tabular}

\begin{tabular}{|c|c|}
\hline \multicolumn{2}{|c|}{ 4" Wood Walls } \\
\hline Nuclide & Mass Density $\left(\mathrm{g} / \mathrm{cm}^{3}\right)$ \\
C (nat.) & $5.3328 \mathrm{E}-02$ \\
${ }^{1} \mathrm{H}$ & $7.4640 \mathrm{E}-03$ \\
${ }^{16} \mathrm{O}$ & $5.9208 \mathrm{E}-02$ \\
\hline
\end{tabular}




\begin{tabular}{|l|c|}
\hline \multicolumn{2}{|c|}{ Basement Ceiling/First Floor-Deck } \\
\hline Nuclide & Mass Fraction \\
${ }^{27} \mathrm{Al}$ & 0.03177 \\
$\mathrm{Ca}$ (nat.) & 0.04112 \\
$\mathrm{Fe}$ (nat.) & 0.06160 \\
${ }^{1} \mathrm{H}$ & 0.00934 \\
${ }^{16} \mathrm{O}$ & 0.49712 \\
$\mathrm{Si}$ (nat.) & 0.31491 \\
${ }^{23} \mathrm{Na}$ & 0.02710 \\
$\mathrm{Cr}$ (nat.) & 0.01180 \\
$\mathrm{Ni}$ (nat.) & 0.00524 \\
The density of the mixture is $2.4121 \mathrm{~g} / \mathrm{cm}^{3}$. \\
\hline
\end{tabular}

\begin{tabular}{|l|c|}
\hline \multicolumn{2}{|c|}{ First Floor-Ceiling/Second Floor-Deck } \\
\hline Nuclide & Mass Fraction \\
${ }^{27} \mathrm{Al}$ & 0.00454 \\
${ }^{10} \mathrm{~B}$ & 0.01680 \\
${ }^{16} \mathrm{O}$ & 0.24290 \\
${ }^{\mathrm{Si}(\text { nat.) }}$ & 0.1712 \\
${ }^{23} \mathrm{Na}$ & 0.01861 \\
$\mathrm{Fe}$ (nat.) & 0.40400 \\
$\mathrm{Cr}$ (nat.) & 0.09828 \\
$\mathrm{Ni}$ (nat.) & 0.04368 \\
The density of the mixture is $0.47511 \mathrm{~g} / \mathrm{cm}^{3}$. \\
\hline
\end{tabular}


Full-Office Material Composition

The typical full office has one desk, one file cabinet, and three bookshelves. The dimensions are as follows:

$$
\begin{array}{ll}
\text { Desk }=36 ” * 15 ” * 26 ” & \mathrm{Vd}=14,040 \text { inches3 } \\
\text { File Cabinet }=60 ” * 15 ” * 26 ” & \mathrm{Vfc}=23,400 \text { inches3 } \\
\text { Bookshelf }=82 ” * 36 ” * 12 ” & \mathrm{Vs}=35,424 \text { inches3 }
\end{array}
$$

Therefore, the total volume of the wood in the room is Vd $+\mathrm{Vfc}+3 \mathrm{Vs}$, which is $143,712 \mathrm{in}^{3}$ or $2.35502 \mathrm{E}+6 \mathrm{~cm}^{3}$.

The percent volume of wood $=\mathrm{Vw} /$ Vroom $=4.85496 \mathrm{E}-02$

Wood is assumed to be cellulose $\left(\mathrm{C}_{6} \mathrm{H}_{10} \mathrm{O}_{5}\right)$ at $1 \mathrm{~g} / \mathrm{cm}^{3}$.

Therefore, the weight fractions are

$$
\begin{array}{ll}
\mathrm{C}= & 0.4444 \\
\mathrm{H}= & 0.0622 \\
\mathrm{O}= & 0.4934
\end{array}
$$

The material densities are therefore:

$$
\begin{aligned}
& \mathrm{C}=2.15754 \mathrm{E}-02 \mathrm{~g} / \mathrm{cm}^{3} \\
& \mathrm{H}=3.01979 \mathrm{E}-03 \mathrm{~g} / \mathrm{cm}^{3} \\
& \mathrm{O}=2.39544 \mathrm{E}-02 \mathrm{~g} / \mathrm{cm}^{3}
\end{aligned}
$$

Total Room Density $=4.855 \mathrm{E}-02 \mathrm{~g} / \mathrm{cm}^{3}$ 
Full-Laboratory Material Composition

$$
\begin{aligned}
\text { Room Volume } & =(802.64 \mathrm{~cm} * 601.98 \mathrm{~cm} * 441.96 \mathrm{~cm}) \\
& - \\
& (220.98 \mathrm{~cm} * 60.96 \mathrm{~cm} * 441.96 \mathrm{~cm}) \\
& =2.0164 \mathrm{E}+08 \mathrm{~cm}^{3}
\end{aligned}
$$

Glove Box

SS panels

$\begin{array}{rlllcl}\text { Number } & \mathrm{x} & \mathrm{y} & \mathrm{z} & \text { Volume }\left(\mathrm{in}^{3}\right) & \text { Volume }\left(\mathrm{cm}^{3}\right) \\ 2 & 24 & 48 & 0.25 & 576 & 9,438.95 \\ 2 & 48 & 36 & 0.25 & 864 & 14,158.42 \\ 2 & 36 & 24 & 0.25 & 432 & 7,079.212\end{array}$

Glass panels

$\begin{array}{rlllcl}\text { Number } & \mathrm{x} & \mathrm{y} & \mathrm{z} & \text { Volume }\left(\mathrm{in}^{3}\right) & \text { Volume }\left(\mathrm{cm}^{3}\right) \\ 2 & 48 & 36 & 0.25 & 864 & 14,158.42 \\ 2 & 24 & 36 & 0.25 & 432 & 7,079.212\end{array}$

Lead Bricks

$\begin{array}{rrrrcl}\text { Number } & \mathrm{x} & \mathrm{y} & \mathrm{z} & \text { Volume }\left(\mathrm{in}^{3}\right) & \text { Volume }\left(\mathrm{cm}^{3}\right) \\ 6 & 2 & 4 & 8 & 384 & 6,292.633\end{array}$

Stainless Steel Sheet to Represent Instruments

$\begin{array}{rlrll}\text { Number } & \mathrm{x} & \mathrm{y} & \mathrm{z} & \text { Volume }\left(\mathrm{cm}^{3}\right) \\ 1 & 802.64601 .981 .0 & 483,173\end{array}$

\begin{tabular}{|c|c|c|}
\hline Number & $\mathrm{y}$ & Volume $\left(\mathrm{cm}^{3}\right)$ \\
\hline 1 & 802.64601 .981 .0 & 483,173 \\
\hline
\end{tabular}

Glass Sheet to Represent Instruments

$\begin{array}{rllll}\text { Number } & \mathrm{x} & \mathrm{y} & \mathrm{z} & \text { Volume }\left(\mathrm{cm}^{3}\right) \\ 1 & 802.64601 .98 & 1.0 & 483,173\end{array}$

Wood Sheet to Represent Tables and Desks 
Two Bookshelves

Two File Cabinets

Material Totals

$1,160,991 \mathrm{~cm}^{3}$

$766,915 \mathrm{~cm}^{3}$

Volume Fraction Density in Room $\left(\mathrm{g} / \mathrm{cm}^{3}\right)$

Glass

504,410

2.5016E-03

5.5785E-03

SS

513,849

2.5484E-03

2.0209E-02

$\mathrm{Pb}$

6,292

3.1208E-05

3.5421E-04

Wood

2,411,078

1.1958E-02

1.1958E-02

Air

$1.9820 \mathrm{E}+08$

9.8296E-01

1.1815E-03

Densities (Reppond, 1977)

$$
\begin{aligned}
& \text { Glass }=2.32 \mathrm{~g} / \mathrm{cm}^{3} \\
& \mathrm{SS}=7.93 \mathrm{~g} / \mathrm{cm}^{3} \\
& \mathrm{~Pb} \quad=11.35 \mathrm{~g} / \mathrm{cm}^{3} \\
& \text { Wood }=1.00 \mathrm{~g} / \mathrm{cm}^{3} \\
& \text { Air }=0.001202 \mathrm{~g} / \mathrm{cm}^{3}
\end{aligned}
$$




\begin{tabular}{|l|l|}
\hline Nuclide & Weight Fraction \\
\hline $\mathrm{Al}$ & $1.4202 \mathrm{E}-03$ \\
$\mathrm{~B}$ & $5.2546 \mathrm{E}-03$ \\
$\mathrm{Na}$ & $5.8227 \mathrm{E}-03$ \\
$\mathrm{O}$ & $2.3249 \mathrm{E}-01$ \\
$\mathrm{Si}$ & $5.3541 \mathrm{E}-02$ \\
$\mathrm{Fe}$ & $3.8071 \mathrm{E}-01$ \\
$\mathrm{Cr}$ & $9.2605 \mathrm{E}-02$ \\
$\mathrm{Ni}$ & $4.1158 \mathrm{E}-02$ \\
$\mathrm{~Pb}$ & $9.0174 \mathrm{E}-03$ \\
$\mathrm{C}$ & $1.3528 \mathrm{E}-01$ \\
$\mathrm{H}$ & $1.8935 \mathrm{E}-02$ \\
$\mathrm{~N}$ & $2.3762 \mathrm{E}-02$ \\
\hline Total Density & $0.03928 \mathrm{~g} / \mathrm{cm} 3$ \\
\hline
\end{tabular}


Sparse-Laboratory Material Composition

Room Volume $\quad=(802.64 \mathrm{~cm} * 601.98 \mathrm{~cm} * 441.96 \mathrm{~cm})$

$$
\begin{aligned}
& \left(220.98 \mathrm{~cm} * 60.96 \mathrm{~cm}^{*} 441.96 \mathrm{~cm}\right) \\
& =2.0164 \mathrm{E}+08 \mathrm{~cm}^{3}
\end{aligned}
$$

It is assumed that there is one-half the total amount of material that is present in the fulllaboratory model.

$\begin{array}{llll}\text { Material Totals } & \text { Volume }\left(\mathrm{cm}^{3}\right) & \text { Volume Fraction } & \text { Density in } \\ \text { Room } & & & \\ \text { Glass } & 252,205 & 1.2508 \mathrm{E}-03 & 2.7893 \mathrm{E}-03 \\ \mathrm{SS} & 256,924 & 1.2742 \mathrm{E}-03 & 1.0104 \mathrm{E}-02 \\ \mathrm{~Pb} & 3,146 & 1.5604 \mathrm{E}-05 & 1.7710 \mathrm{E}-04 \\ \text { Wood } & 1,205,539 & 5.9788 \mathrm{E}-02 & 5.9788 \mathrm{E}-02 \\ \text { Air } & 1.9992 \mathrm{E}+08 & 9.9148 \mathrm{E}-01 & 1.1918 \mathrm{E}-03\end{array}$

Densities (Reppond, 1977)

$$
\begin{array}{ll}
\text { Glass } & =2.32 \mathrm{~g} / \mathrm{cm}^{3} \\
\text { SS } & =7.93 \mathrm{~g} / \mathrm{cm}^{3} \\
\mathrm{~Pb} & =11.35 \mathrm{~g} / \mathrm{cm}^{3} \\
\text { Wood } & =1.00 \mathrm{~g} / \mathrm{cm}^{3} \\
\text { Air } & =0.001202 \mathrm{~g} / \mathrm{cm}^{3}
\end{array}
$$




\begin{tabular}{|l|l|}
\hline Nuclide & Weight Fraction \\
\hline $\mathrm{Al}$ & $1.3780 \mathrm{E}-03$ \\
$\mathrm{~B}$ & $5.0986 \mathrm{E}-03$ \\
$\mathrm{Na}$ & $5.6498 \mathrm{E}-03$ \\
$\mathrm{O}$ & $2.3183 \mathrm{E}-01$ \\
$\mathrm{Si}$ & $5.1951 \mathrm{E}-02$ \\
$\mathrm{Fe}$ & $3.6941 \mathrm{E}-01$ \\
$\mathrm{Cr}$ & $8.9855 \mathrm{E}-02$ \\
$\mathrm{Ni}$ & $3.9936 \mathrm{E}-02$ \\
$\mathrm{~Pb}$ & $8.7497 \mathrm{E}-03$ \\
$\mathrm{C}$ & $1.3126 \mathrm{E}-01$ \\
$\mathrm{H}$ & $1.8372 \mathrm{E}-02$ \\
$\mathrm{~N}$ & $4.6513 \mathrm{E}-02$ \\
\hline Total Density & $0.020240 \mathrm{~g} / \mathrm{cm}^{3}$ \\
\hline
\end{tabular}




\section{Appendix D: Schedule of Deliverables}

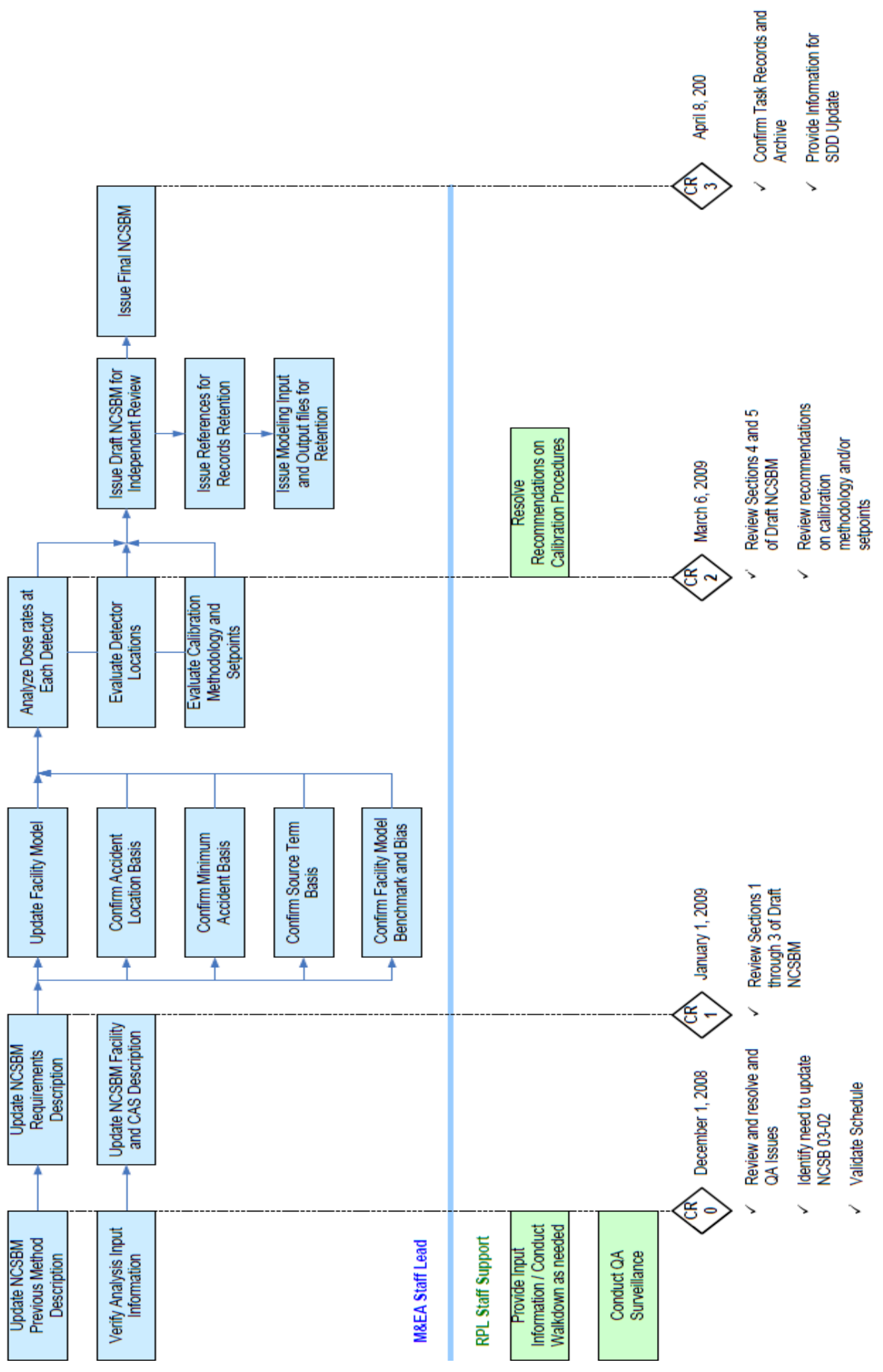




\section{Appendix E: Assumptions Used in the CAS Design of RPL}

\section{Modeling Assumptions}

1) 1st Floor Deck is a sandwiched 14 gauge steel honeycomb with a maximum of 5" of "standard Concrete" The ceiling composition is based on the elevation blue prints listed below, which show that the ceiling is on average 3.75" thick with 14gauge steel (0.0766" thick, Reference 23). This works to a volume ratio of 0.98/0.02 concrete to stainless steel and a mass ratio of $0.934 / 0.066$ concrete to stainless steel.

2) $2^{\text {nd }}$ Floor Deck is a sandwiched 16 gauge steel honeycomb with a maximum of 5 inches of "Emmeshic Concrete". Dave Koontz indicated that this concrete was roughly $30 \%$ if the density of standard concrete.

3) The Gable of all roofs was modified to be a flat equivalent slab at the average height of the actual roof.

4) The ground surrounding 325 was assumed to be $\mathrm{SiO} 2$ with a $66 \%$ packing fraction.

5) Room fill was approached as a combination of full, half-full, or empty office/lab.

6) Dave Koontz mentioned Asbestos in the hollow walls on the 300, 400, and 500 hallways. Have not included any Asbestos in the model because I was unable to verify its existence.

7) The offset of roof parapets were omitted. Walls are capped by the roofs.

8) Basement Deck is set as $1 \mathrm{ft}$ thick from drawing H-4-50013 -Hard to read

9) All staircases omitted

10) Altitudes/elevations are very difficult to verify. This is especially true for HLRF. The original schematics are in PDF format and are extremely cluttered but all non-roof elevations are referenced from H-4-50013-1 and H-3-12901. 
11) The exterior wall of B325 changes from structural concrete to metal siding, insulation, metal support columns, and the occasional window. This change from concrete to metal conglomerate occurs at 404'-6" for the majority of the building. The Northern exterior walls do not change at this height. They change at 416'-4”. For simplicity, I have set the ENTIRE buildings exterior concrete wall to a height of 404'-6”. The material compositions of the exterior wall versus concrete support that making this change is conservative because reflection decreases with the omission of the concrete northern wall.

12) Roof elevations were taken from drawing H-3-305005. The roof in the NW corner of HLRF has been commented out. The roof composition was observed to be a conglomerate of asphalt, tar, gravel, and cement. Because I have found no documents that state the actual contents, I have set all roofs to concrete. All roofs run to the edge of the sections they cover -No overhangs. You will notice a rather large gap above the central section of floor 1 and the corresponding roof. This was done intentionally because there is a large crawl space. The gap is due to setting the roof position to the average height of the gable.

13) The Elevation for the roofs were taken from Drawings H-3-12901 and H-3305005 to be 429 '9" or $13098.8 \mathrm{~cm}$. The roof also has a gable that runs a delta of approximately 6" over the span of the roof over HLRF. The highest point is approximately 430' and the lowest is approximately 429'6".

14) The very small roof over the southern entrance to RPL was neglected.

15) The CAD Drawings clearly show the top floor of HLRF E wall at 230.9' $(586.5 \mathrm{~cm})$ from the SW corner. The second floor CAD drawings clearly show the second floor E wall at 224.4' $(570 \mathrm{~cm})$ from the same origin. I am leaving the dimensions as shown on the CAD drawings until inspection proves otherwise. The point of this statement is there is a noticeable overhang of the second floor HLRF E wall over the same first floor E wall.

16) ALL CHAIN LINK was omitted.

17) There are three vaults $E$ of HLRF that have not been added to the model.

18) Men’s and Women’s restrooms were left empty 
19) Tank 46 was modeled as empty.

20) The corresponding materials for SAL and HLRF hot cells were smeared to be the most limiting conservative cases.

21) From personal inspection, a grade was found in the deck of the Basement mezzanine. The altitude change was observed to be $1 \mathrm{ft}$ over the length of the deck. This slope was ignored and the deck of the mezzanine was held level at $8.5 \mathrm{ft}$. The offset of the deck of the mezzanine to the deck of the central section of the basement was measured at $5 \mathrm{ft}$.

22) The southern mezzanine section of B1 is set $5 \mathrm{ft}$ above the central B1 Deck. This is an observed distance because I have found no blueprint that clearly defines this distance.

23) There is a small gap between Rm23B and the top of Rm32 where there is no sand and just air.

24) There is no sand up to the grade height in the SE corner of the building. This was done to be conservative. There are numerous areas in the SE that do not have a backfill to the same grade as the rest of the building. There is also a loading dock located in the SE corner which is simply free space lined with concrete.

25) The material fill for all interior office/laboratory walls were set to be the same "metal fill" listed in the materials definition because there was no access to the interior of the walls

\section{Translation notes}

The basement was translated in reference to the SW section. Floor1 was translated with reference to the NW section. The second floor was translated in reference to the SW section. TR4=basement translation, TR1=Floor1 translation, TR2=Floor2 translation. And TR3=Floor3 translation. 


\section{Appendix F: MCNP5 Input Decks}

Bldg. 325 RPL 10/15/2008

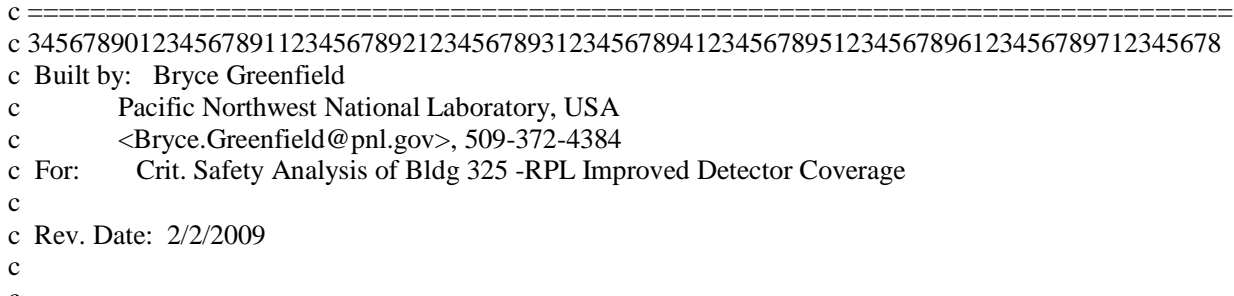

c

c Each Surface block contains a header with notes about that section.

c Whenever possible and or convenient the origin for each sections coordinate system

c was place in the most Southwestern point in that section. On some junctions between surfaces

c boundary errors arose even though the dimensions were correctly entered. The solution

c used to eliminate these errors was to back out the conflicting boundary by a very small margin $0.01 \mathrm{~cm}$.

c Each surface is followed by a brief description of what and where it is. Surfacs were modeled,

c grouped, and numbered according to their respective room numbers. The format for both the cell

c and surface cards is as follows (\#\#\#\$). The \#\#\# signs are the room numbers with a

c range of 1-999 and the $\$$ signs are the surface/cell numbers that range from 1-99.

c For example 701 refers to room 7 surface 1 . Wheras 7001 refers to room 70 surface 1 .

c Cardinal directions relative to each room were used to roughly

c describe where a surface existed. For example: $\mathrm{W}$ wall Rm72.

c Hopefully it is easy to interpret as the western wall of Room 72. When multiple surfaces

c existed in the same room/plane they were described with SW, CentralW, or NW. If there were numerous

c bodies in the same room/plane then a numbering system was used that always started with the

c southwestern most body and the moved eastward. After a terminating surface was encountered

c the numbering was continued by moving back to the starting body(the most SW body) and moving North

c to continue the numbering sequence. For an example of this check the descriptions of the columns

c modeled in Room 45 of the Basement.

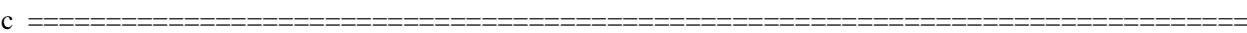

Index of Section Identifier Tags <-Use Search to navigate through file

TAG=TAGb1roomfill - B1 Room fills

TAG=TAGf1roomfill - Floor 1 Room fills

TAG=TAGf2roomfill - Floor 2 Room fills

TAG=TAGPuPu - Accident Surfaces/Locations

c TAG=TAGdetectors - Detector surfaces/Locations

c TAG=TAGbasementsurfaces - B1 surfaces

c TAG=TAGfloor1surfaces - 1st floor surfaces

c TAG=TAGfloor2surfaces - 2nd floor surfaces

c TAG $=$ TAGfloor3surfaces - 3rd floor surfaces

c TAG $=$ TAGmaterials - Materials definition

c TAG=TAGsourcedef - Accident Locations Specific SDEF

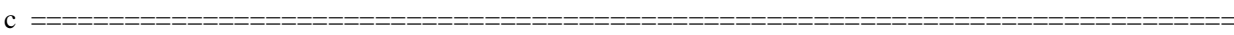

Legend of Abbreviations

c o = outside

c $\mathrm{i}=$ inside

c $\mathrm{wl}=$ wall

c $\mathrm{Rm}=\mathrm{Room}$

c $\mathrm{LR}=$ Lunch Room

c $\mathrm{u}=$ upper

c $\mathrm{l}=$ lower

c $\mathrm{N}=$ North 


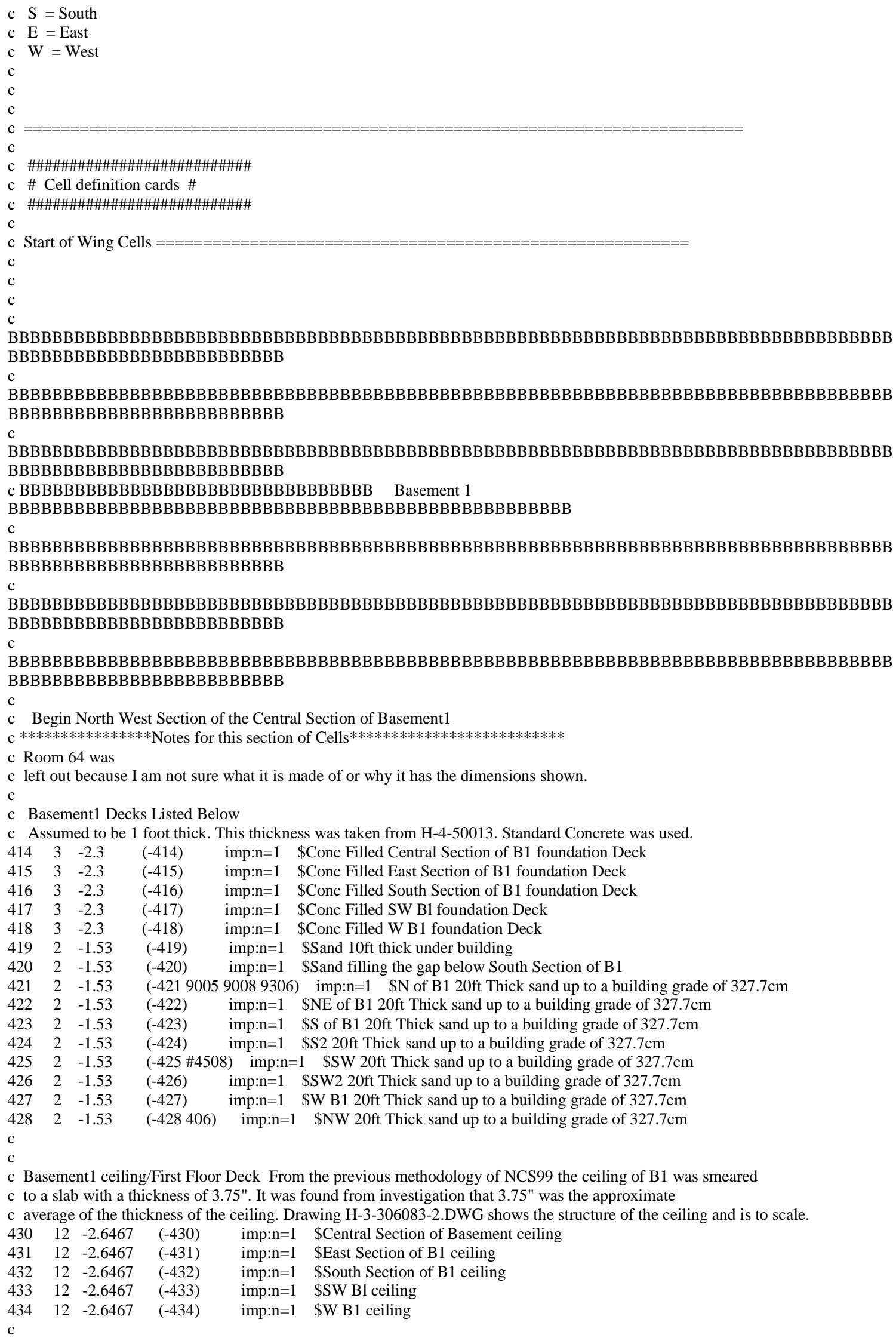




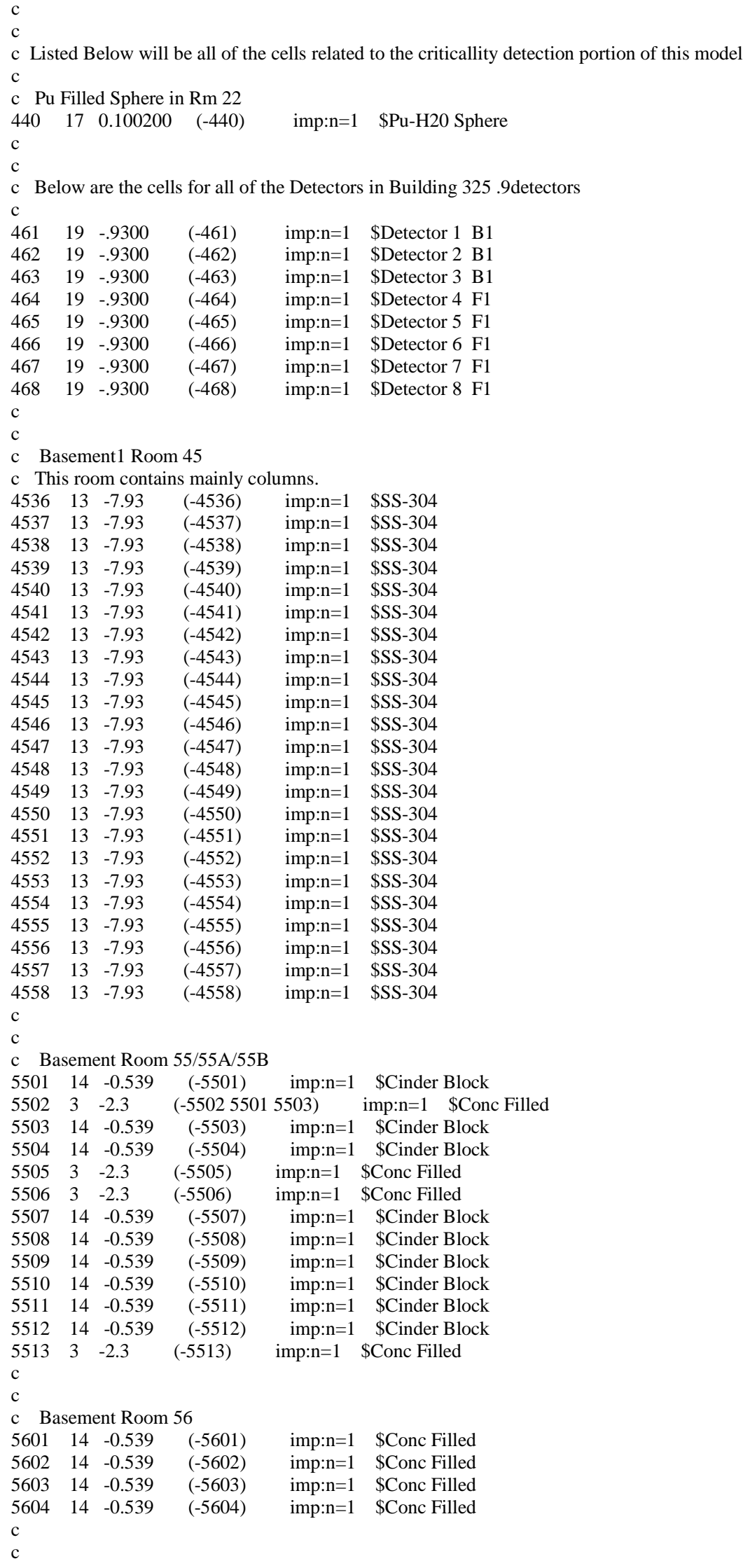




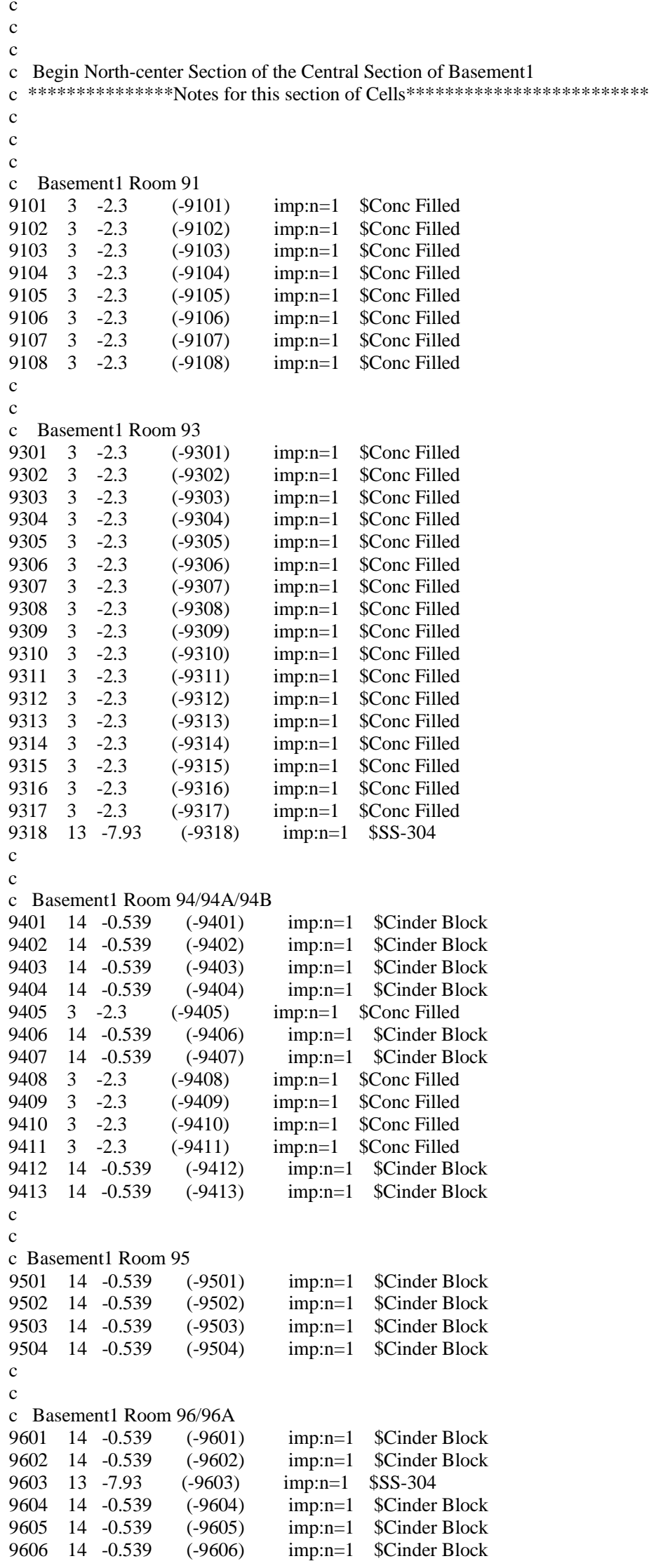




\begin{tabular}{|c|c|c|c|c|c|}
\hline \multirow{2}{*}{\multicolumn{6}{|c|}{ \$Cinder Block }} \\
\hline & & & & & \\
\hline \multicolumn{6}{|l|}{ C } \\
\hline \multicolumn{6}{|c|}{ c Basement1 Room 97/97A } \\
\hline 9701 & 14 & -0.539 & $(-9701)$ & imp:n=1 & \$Cinder Block \\
\hline 9702 & 3 & -2.3 & $(-9702)$ & imp:n=1 & \$Conc Filled \\
\hline 9703 & 14 & -0.539 & $(-9703)$ & imp:n=1 & \$Cinder Block \\
\hline 9704 & 13 & -7.93 & $(-9704)$ & imp:n=1 & \$SS-304 \\
\hline 9705 & 14 & -0.539 & $(-9705)$ & imp:n=1 & \$Cinder Block \\
\hline 9706 & 14 & -0.539 & $(-9706)$ & imp:n=1 & \$Cinder Block \\
\hline 9707 & 13 & -7.93 & $(-9707)$ & imp:n=1 & \$SS-304 \\
\hline \multicolumn{6}{|l|}{ C } \\
\hline \multicolumn{6}{|l|}{ C } \\
\hline \multicolumn{6}{|c|}{ c Basement Room 98} \\
\hline 9801 & 14 & -0.539 & $(-9801)$ & imp:n=1 & \$Cinder Block \\
\hline \multicolumn{6}{|l|}{ c } \\
\hline \multicolumn{6}{|l|}{ C } \\
\hline \multicolumn{6}{|c|}{ c Basement Room 45} \\
\hline 4560 & 3 & -2.3 & $(-4560)$ & imp:n=1 & \$Conc Filled \\
\hline 4561 & 3 & -2.3 & $(-4561)$ & imp:n=1 & \$Conc Filled \\
\hline 4562 & 3 & -2.3 & $(-4562)$ & imp:n=1 & \$Conc Filled \\
\hline 4563 & 3 & -2.3 & $(-4563)$ & imp:n=1 & \$Conc Filled \\
\hline 4564 & 13 & -7.93 & $(-4564)$ & imp:n=1 & \$SS-304 \\
\hline 4565 & 13 & -7.93 & $(-4565)$ & imp:n=1 & \$SS-304 \\
\hline 4566 & 3 & -2.3 & $(-4566)$ & imp:n=1 & \$Conc Filled \\
\hline 4567 & 3 & -2.3 & $(-4567)$ & imp:n=1 & \$Conc Filled \\
\hline 4568 & 3 & -2.3 & $(-4568)$ & imp:n=1 & \$Conc Filled \\
\hline 4569 & 3 & -2.3 & $(-4569)$ & imp:n=1 & \$Conc Filled \\
\hline с 4570 & 3 & -2.3 & $(-4570)$ & imp:n=1 & \$Conc Filled \\
\hline с 4571 & 3 & -2.3 & $(-4571)$ & imp:n=1 & \$Conc Filled \\
\hline \multicolumn{6}{|l|}{ c } \\
\hline \multicolumn{6}{|l|}{ C } \\
\hline c Bas & eme & nt Room & 90/Elev Ro & & \\
\hline 9001 & 3 & -2.3 & $(-9001)$ & imp:n=1 & \$Conc Filled \\
\hline 9002 & 3 & -2.3 & $(-9002)$ & imp:n=1 & \$Conc Filled \\
\hline 9003 & 3 & -2.3 & $(-9003)$ & imp:n=1 & \$Conc Filled \\
\hline 9004 & 3 & -2.3 & $(-9004)$ & imp:n=1 & \$Conc Filled \\
\hline 9005 & 3 & -2.3 & $(-9005)$ & imp:n=1 & \$Conc Filled \\
\hline 9006 & 3 & -2.3 & $(-9006)$ & imp:n=1 & \$Conc Filled \\
\hline 9007 & 3 & -2.3 & $(-9007)$ & imp:n=1 & \$Conc Filled \\
\hline 9008 & 3 & -2.3 & $(-9008)$ & imp:n=1 & \$Conc Filled \\
\hline 9009 & 3 & -2.3 & $(-9009)$ & imp:n=1 & \$Conc Filled \\
\hline 9010 & 3 & -2.3 & $(-9010)$ & imp:n=1 & \$Conc Filled \\
\hline 9011 & 3 & -2.3 & $(-9011)$ & imp:n=1 & \$Conc Filled \\
\hline 9012 & 3 & -2.3 & $(-9012)$ & imp:n=1 & \$Conc Filled \\
\hline 9013 & 3 & -2.3 & $(-9013)$ & imp:n=1 & \$Conc Filled \\
\hline 9014 & 3 & -2.3 & $(-9014)$ & imp:n=1 & \$Conc Filled \\
\hline 9015 & 3 & -2.3 & $(-9015)$ & imp:n=1 & \$Conc Filled \\
\hline 9016 & 3 & -2.3 & $(-9016)$ & imp:n=1 & \$Conc Filled \\
\hline C & & & & & \\
\hline C & & & & & \\
\hline C & & & & & \\
\hline C & & & & & \\
\hline C & & & & & \\
\hline c $\mathrm{Be}$ & $\operatorname{gin} 1$ & Northeas & t Section of & e Central Se & ection of Basement1 \\
\hline $\mathrm{c} * *$ & & & $* * *$ Notes $\mathrm{f}$ & this section & of Cells $* * * * * * * * * * * * * * * * * * * * * *$ \\
\hline c & & & & & \\
\hline C & & & & & \\
\hline c Bas & eme & nt Room & & & \\
\hline 4575 & 13 & -7.93 & $(-4575)$ & imp:n=1 & \$SS-304 \\
\hline 4576 & 13 & -7.93 & $(-4576)$ & imp:n=1 & \$SS-304 \\
\hline 4577 & 13 & -7.93 & $(-4577)$ & imp:n=1 & \$SS-304 \\
\hline 4578 & 13 & -7.93 & $(-4578)$ & imp:n=1 & \$SS-304 \\
\hline 4579 & 13 & -7.93 & $(-4579)$ & imp:n=1 & \$SS-304 \\
\hline 4580 & 13 & -7.93 & $(-4580)$ & imp:n=1 & \$SS-304 \\
\hline 4581 & 13 & -7.93 & $(-4581)$ & imp:n=1 & \$SS-304 \\
\hline 4582 & 13 & -7.93 & $(-4582)$ & imp:n=1 & \$SS-304 \\
\hline 4583 & 13 & -7.93 & $(-4583)$ & imp:n=1 & \$SS-304 \\
\hline C & & & & & \\
\hline$C$ & & & & & \\
\hline
\end{tabular}




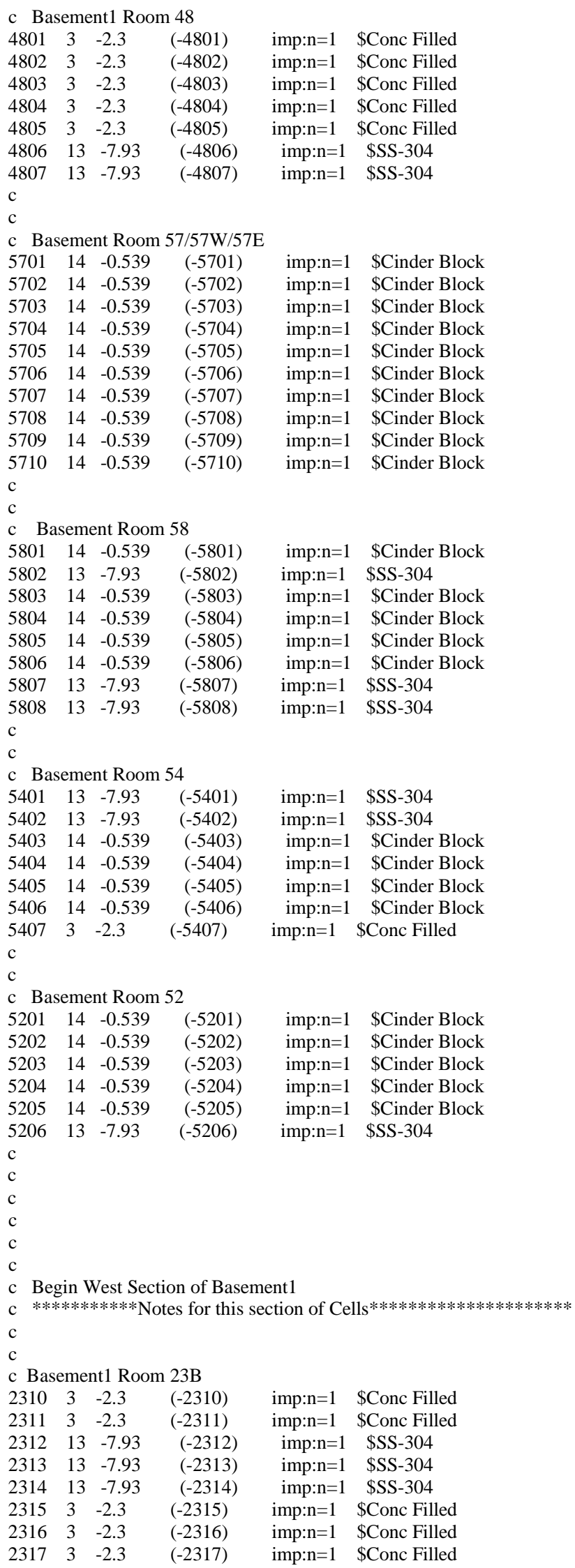




\begin{tabular}{|c|c|c|c|c|c|}
\hline 2318 & 13 & -7.93 & $(-2318)$ & imp:n=1 & \$SS-304 \\
\hline \multirow{2}{*}{$\begin{array}{l}2319 \\
2320\end{array}$} & 13 & -7.93 & $(-2319)$ & imp: $n=1$ & \$SS-304 \\
\hline & 13 & -7.93 & $(-2320)$ & imp: $n=1$ & \$SS-304 \\
\hline \multicolumn{6}{|l|}{ C } \\
\hline \multicolumn{6}{|c|}{$\mathrm{C}$} \\
\hline \multicolumn{6}{|c|}{ c Basement1 Room 45} \\
\hline 4501 & 3 & -2.3 & $(-4501)$ & imp:n=1 & \$Conc Filled \\
\hline 4502 & 3 & -2.3 & $(-4502)$ & imp:n=1 & \$Conc Filled \\
\hline 4503 & 3 & -2.3 & $(-4503)$ & imp:n=1 & \$Conc Filled \\
\hline 4504 & 3 & -2.3 & $(-4504)$ & imp:n=1 & \$Conc Filled \\
\hline 4505 & 3 & -2.3 & $(-4505)$ & imp:n=1 & \$Conc Filled \\
\hline 4506 & 3 & -2.3 & $(-4506)$ & imp: $n=1$ & \$Conc Filled \\
\hline 4507 & 3 & -2.3 & $(-4507)$ & imp: $n=1$ & \$Conc Filled \\
\hline 4508 & 3 & -2.3 & $(-4508)$ & imp:n=1 & \$Conc Filled \\
\hline 4509 & 3 & -2.3 & $(-4509)$ & imp:n=1 & \$Conc Filled \\
\hline \multicolumn{6}{|l|}{ c } \\
\hline \multicolumn{6}{|l|}{$\mathrm{C}$} \\
\hline \multicolumn{6}{|c|}{ c Basement Room 32} \\
\hline 3201 & 13 & -7.93 & $(-3201)$ & imp:n=1 & \$SS-304 \\
\hline 3202 & 13 & -7.93 & $(-3202)$ & imp:n=1 & \$SS-304 \\
\hline 3203 & 13 & -7.93 & $(-3203)$ & imp:n=1 & \$SS-304 \\
\hline 3204 & 3 & -2.3 & $(-3204)$ & imp:n=1 & \$Conc Filled \\
\hline 3205 & 3 & -2.3 & $(-3205)$ & imp:n=1 & \$Conc Filled \\
\hline 3206 & 3 & -2.3 & $(-3206)$ & imp:n=1 & \$Conc Filled \\
\hline 3207 & 3 & -2.3 & $(-3207)$ & imp: $n=1$ & \$Conc Filled \\
\hline 3208 & 3 & -2.3 & $(-3208)$ & imp:n=1 & \$Conc Filled \\
\hline 3209 & 3 & -2.3 & $(-3209)$ & imp:n=1 & \$Conc Filled \\
\hline 3210 & 3 & -2.3 & $(-3210)$ & imp:n=1 & \$Conc Filled \\
\hline 3211 & 3 & -2.3 & $(-3211)$ & imp: $n=1$ & \$Conc Filled \\
\hline 3212 & 3 & -2.3 & $(-3212)$ & imp:n=1 & \$Conc Filled \\
\hline 3213 & 13 & -7.93 & $(-3213)$ & imp:n=1 & \$SS-304 \\
\hline 3214 & 3 & -2.3 & $(-3214)$ & imp:n=1 & \$Conc Filled \\
\hline 3215 & 3 & -2.3 & $(-3215)$ & imp: $n=1$ & \$Conc Filled \\
\hline 3216 & 3 & -2.3 & $(-3216)$ & imp:n=1 & \$Conc Filled \\
\hline 3217 & 3 & -2.3 & $(-3217)$ & imp:n=1 & \$Conc Filled \\
\hline \multicolumn{6}{|l|}{ c } \\
\hline \multicolumn{6}{|l|}{ c } \\
\hline \multicolumn{6}{|l|}{ C } \\
\hline C & & & & & \\
\hline c & & & & & \\
\hline $\mathrm{Be}$ & $\operatorname{gin}$ & South V & est Section & the Central & Section of Basement1 \\
\hline $\mathrm{C} * * *$ & & $* * * * * *$ & $k * * *$ Notes & r this sectio & n of Cells $* * * * * * * * * * * * * * * *$ \\
\hline c & & & & & \\
\hline c & & & & & \\
\hline $\mathrm{c} \mathrm{Ba}$ & sem & ent Roo & 22 & & \\
\hline 2201 & 13 & -7.93 & $(-2201)$ & imp:n=1 & \$SS-304 \\
\hline 2202 & 13 & -7.93 & $(-2202)$ & imp:n=1 & \$SS-304 \\
\hline 2203 & 13 & -7.93 & $(-2203)$ & imp:n=1 & \$SS-304 \\
\hline 2204 & 3 & -2.3 & $(-2204)$ & imp:n=1 & \$Conc Filled \\
\hline 2205 & 13 & -7.93 & $(-2205)$ & imp:n=1 & \$SS-304 \\
\hline 2206 & 13 & -7.93 & $(-2206)$ & imp:n=1 & \$SS-304 \\
\hline 2207 & 3 & -2.3 & $(-2207)$ & imp:n=1 & \$Conc Filled \\
\hline 2208 & 13 & -7.93 & $(-2208)$ & imp:n=1 & \$SS-304 \\
\hline 2209 & 3 & -2.3 & $(-2209)$ & imp:n=1 & \$Conc Filled \\
\hline 2210 & 3 & -2.3 & $(-2210)$ & imp:n=1 & \$Conc Filled \\
\hline 2211 & 3 & -2.3 & $(-2211)$ & imp:n=1 & \$Conc Filled \\
\hline 2212 & 13 & -7.93 & $(-2212)$ & imp:n=1 & \$SS-304 \\
\hline 2213 & 3 & -2.3 & $(-2213)$ & imp:n=1 & \$Conc Filled \\
\hline 2214 & 13 & -7.93 & $(-2214)$ & imp:n=1 & \$SS-304 \\
\hline 2215 & 3 & -2.3 & $(-2215)$ & imp:n=1 & \$Conc Filled \\
\hline 2216 & 3 & -2.3 & $(-2216)$ & imp:n=1 & \$Conc Filled \\
\hline 2217 & 3 & -2.3 & $(-2217)$ & imp:n=1 & \$Conc Filled \\
\hline с 2218 & 3 & -2.3 & $(-2218)$ & imp:n=1 & \$Conc Filled \\
\hline 2219 & 3 & -2.3 & $(-2219)$ & imp:n=1 & \$Conc Filled \\
\hline 2220 & 3 & -2.3 & $(-2220)$ & imp:n=1 & \$Conc Filled \\
\hline 2221 & 3 & -2.3 & $(-2221)$ & imp:n=1 & \$Conc Filled \\
\hline C & & & & & \\
\hline C & & & & & \\
\hline c $\mathrm{Ne}$ & $\mathrm{w} \mathrm{HC}$ & ot Cells & & & \\
\hline 2230 & 13 & -7.93 & $(-2230)$ & imp:n=1 & \$SW Mod Cell-1 Rm22 \\
\hline
\end{tabular}




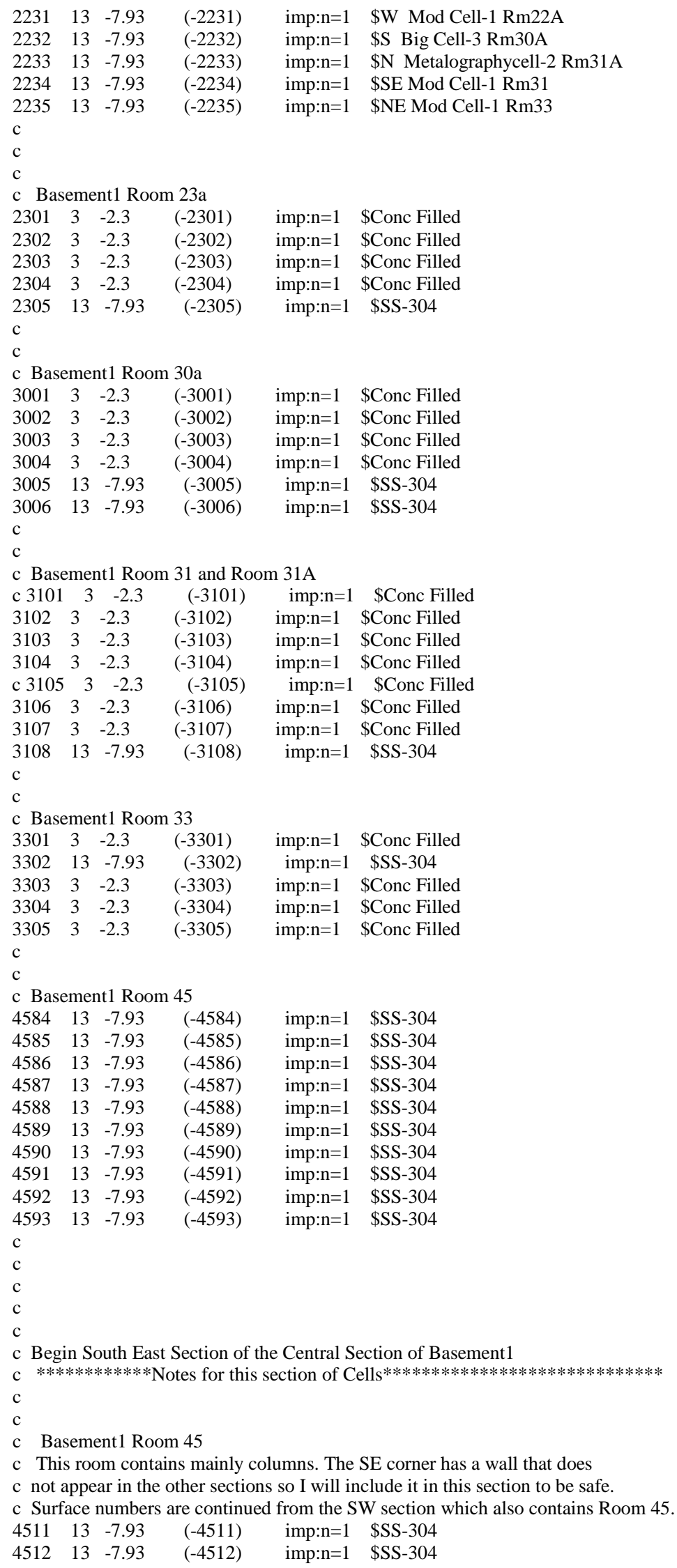




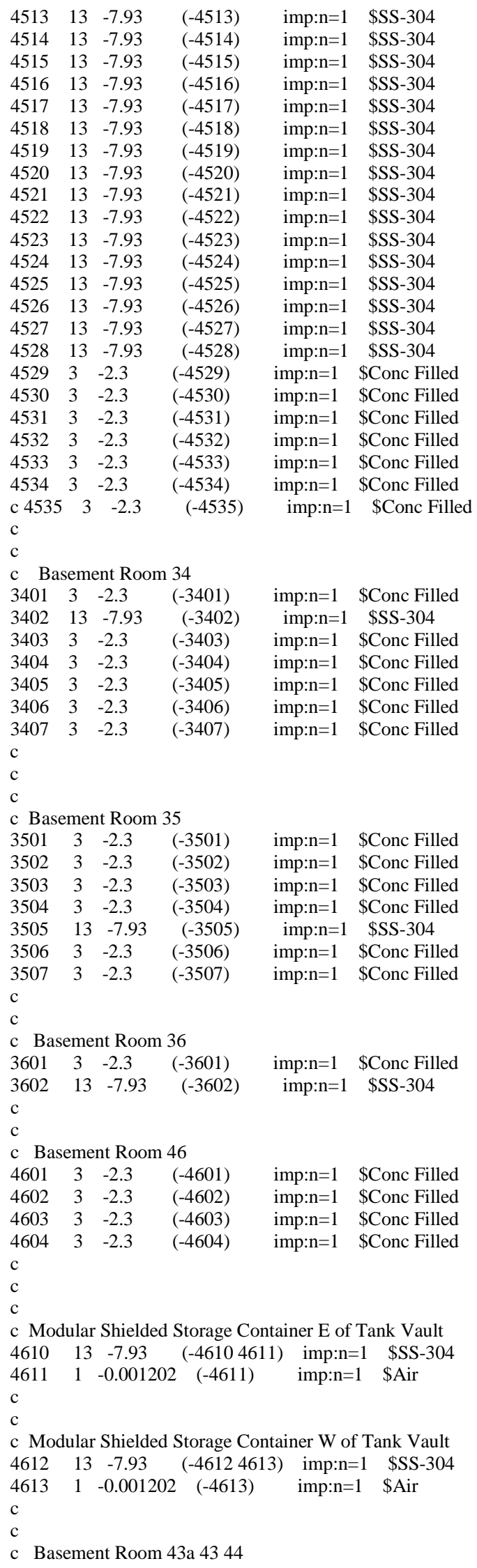




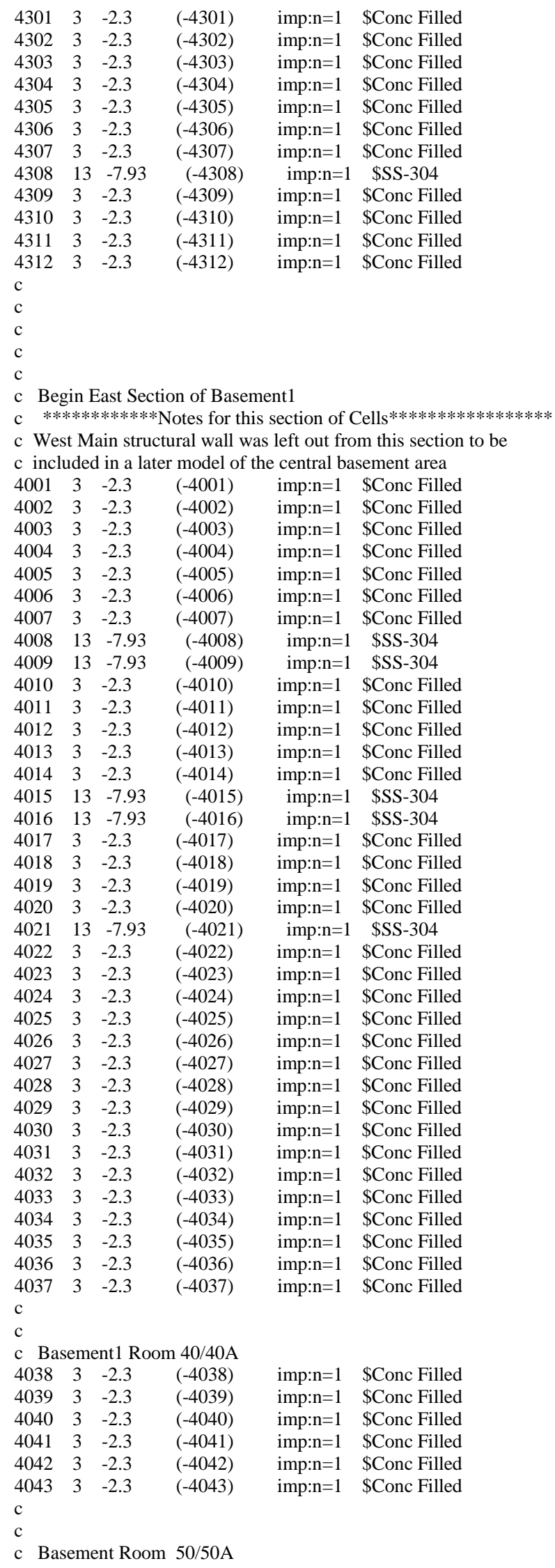




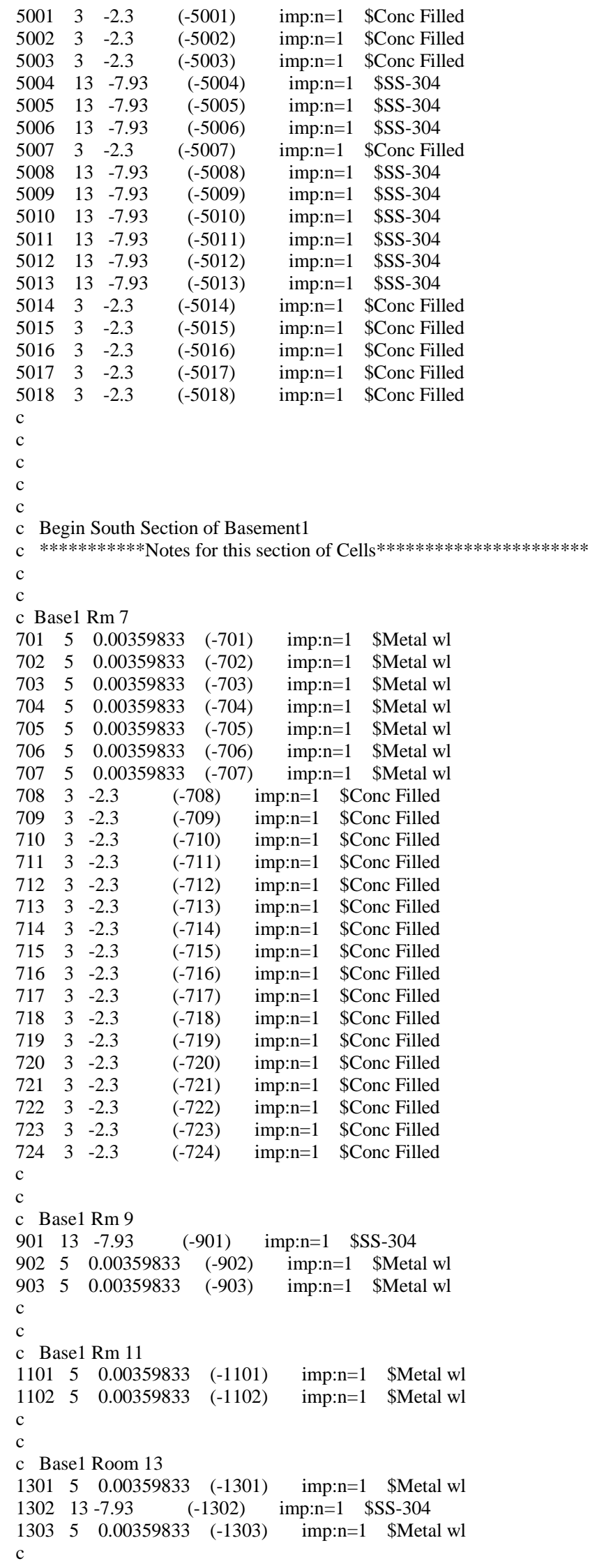


c Base1 Room 15

$150150.00359833 \quad(-1501) \quad$ imp:n=1 \$Metal wl

$150250.00359833 \quad(-1502) \quad$ imp:n=1 \$Metal wl

C

C Base1 Room 17

$\begin{array}{llll}1701 & 5 & 0.00359833 & (-1701)\end{array}$

$\begin{array}{llll}1702 & 5 & 0.00359833 & (-1702)\end{array}$

$\begin{array}{llll}1703 & 5 & 0.00359833 & (-1703)\end{array}$

C

c Base1 Room 16

$\begin{array}{llll}1601 & 5 & 0.00359833 & (-1601)\end{array}$

$\begin{array}{llll}1602 & 5 & 0.00359833 & (-1602)\end{array}$

$\begin{array}{llll}1603 & 5 & 0.00359833 & (-1603)\end{array}$

$\begin{array}{llll}1604 & 5 & 0.00359833 & (-1604)\end{array}$

$\begin{array}{llll}1605 & 5 & 0.00359833 & (-1605)\end{array}$

c Base1 Room 18

$180150.00359833 \quad(-1801) \quad$ imp:n=1 \$Metal wl

$180250.00359833 \quad(-1802) \quad$ imp:n=1 \$Metal wl

C

c Base1 Room 20

$\begin{array}{llll}2001 & 5 & 0.00359833 & (-2001)\end{array}$

$200250.00359833 \quad(-2002)$

$2003 \quad 5 \quad 0.00359833 \quad(-2003)$

C

c Base1 Room

$\begin{array}{llll}2004 & 5 & 0.00359833 & (-2004)\end{array}$

$2005 \quad 5 \quad 0.00359833 \quad(-2005)$

$\begin{array}{llll}2006 & 5 & 0.00359833 & (-2006)\end{array}$

$\begin{array}{llll}2007 & 5 & 0.00359833 & (-2007)\end{array}$

$\begin{array}{llll}2008 & 5 & 0.00359833 & (-2008)\end{array}$

$\begin{array}{llll}2009 & 5 & 0.00359833 & (-2009)\end{array}$

c Base1 Room 26

$\begin{array}{llll}2601 & 5 & 0.00359833 & (-2601)\end{array}$

$\begin{array}{llll}2602 & 5 & 0.00359833 & (-2602)\end{array}$

c Base1 Room 28

$\begin{array}{llll}2801 & 5 & 0.00359833 & (-2801)\end{array}$

$\begin{array}{llll}2802 & 5 & 0.00359833 & (-2802)\end{array}$

$\begin{array}{llll}2803 & 5 & 0.00359833 & (-2803)\end{array}$

C

C

c Base1 Room 70

$700150.00359833 \quad(-7001) \quad$ imp:n=1 \$Metal wl

$700250.00359833 \quad(-7002) \quad$ imp:n=1 \$Metal wl

$700313-7.93 \quad(-7003) \quad$ imp:n=1 \$SS-304

$700450.00359833 \quad(-7004) \quad$ imp:n=1 \$Metal wl

C

c Base1 Room 72

$720150.00359833 \quad(-7201) \quad$ imp:n=1 \$Metal wl

$720250.00359833 \quad(-7202) \quad$ imp:n=1 \$Metal wl

c Base1 Room

$740150.00359833 \quad(-7401) \quad$ imp:n=1 \$Metal wl

$740250.00359833 \quad(-7402) \quad$ imp:n=1 \$Metal wl

$7403 \quad 13 \quad-7.93 \quad(-7403) \quad$ imp:n=1 $\quad$ \$SS-304

c Base1 Room 


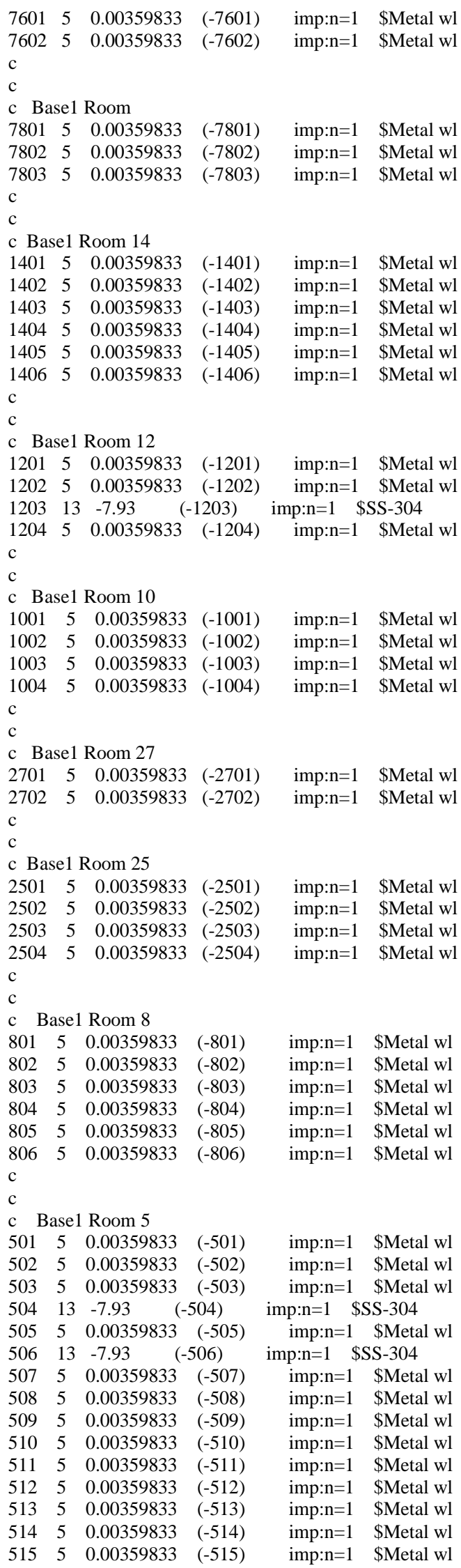




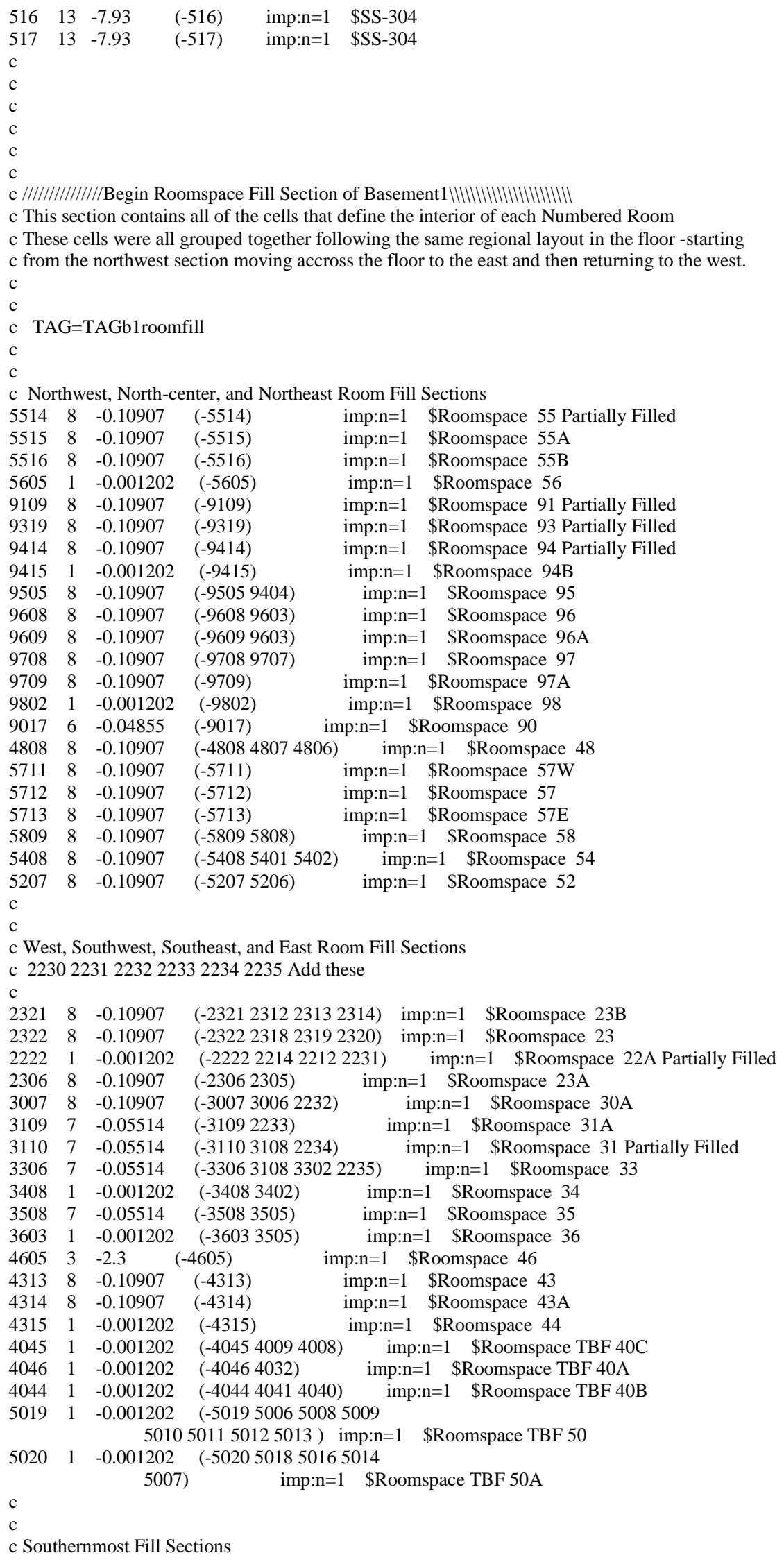




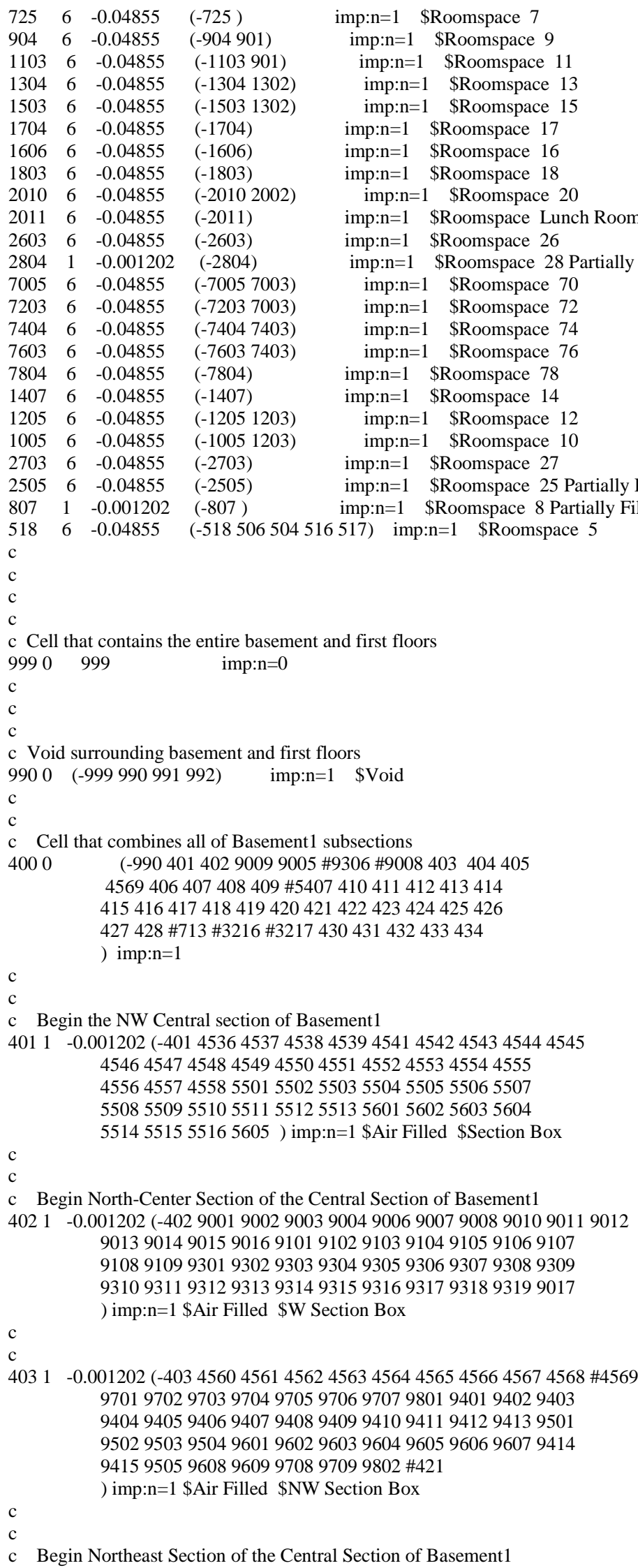




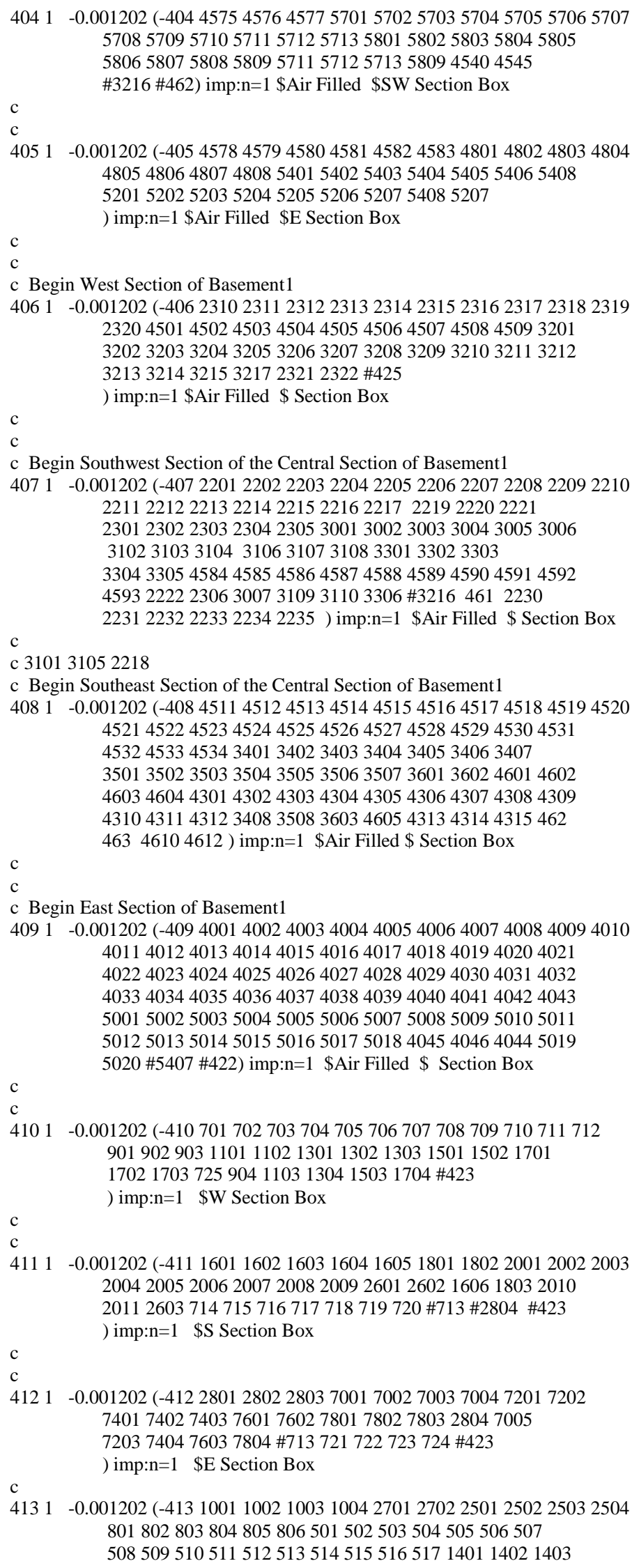


14041405140612011202120312041407120510052703

2505807518 ) imp:n=1 \$Central Section Box

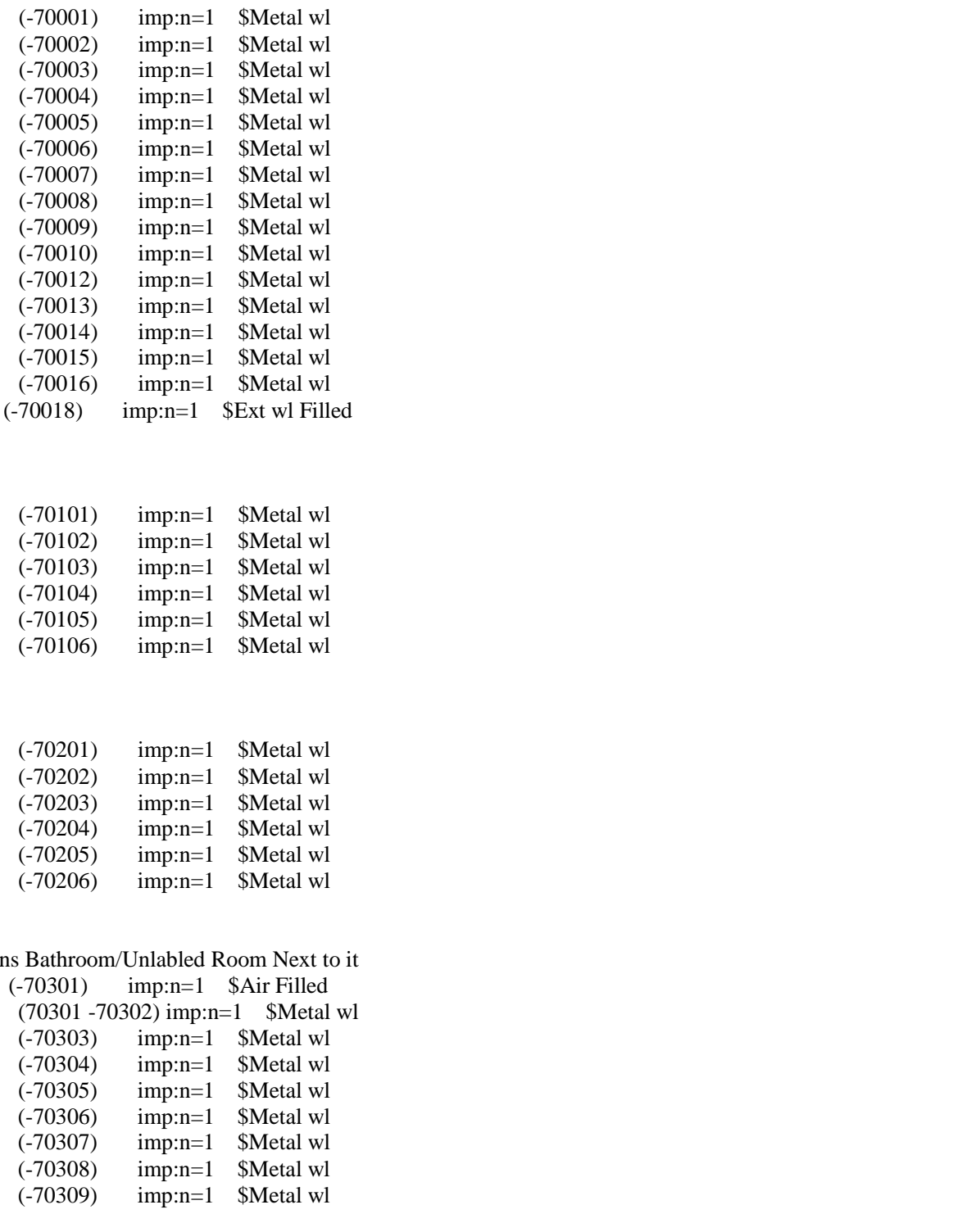




\begin{tabular}{|c|c|c|c|c|c|}
\hline 70310 & 5 & 0.00359833 & $(-70310)$ & imp: $n=1$ & \$Metal wl \\
\hline 70311 & 5 & 0.00359833 & $(-70311)$ & imp: $n=1$ & \$Metal wl \\
\hline 70312 & 5 & 0.00359833 & $(-70312)$ & imp:n=1 & \$Metal wl \\
\hline 70313 & 5 & 0.00359833 & $(-70313)$ & imp:n=1 & \$Metal wl \\
\hline 70314 & 5 & 0.00359833 & $(-70314)$ & imp:n=1 & \$Metal wl \\
\hline 70315 & 5 & 0.00359833 & $(-70315)$ & imp:n=1 & \$Metal wl \\
\hline \\
\hline \\
\hline \multicolumn{6}{|c|}{ c Floor1 Room 320/421 } \\
\hline \multicolumn{2}{|c|}{320015} & 0.00359833 & $(-32001)$ & imp:n=1 & \$Air Filled \\
\hline \multicolumn{2}{|c|}{320025} & 0.00359833 & $(-32002)$ & imp:n=1 & \$Metal wl \\
\hline 32003 & 5 & 0.00359833 & $(-32003)$ & imp:n=1 & \$Metal wl \\
\hline 32004 & 5 & 0.00359833 & $(-32004)$ & imp: $n=1$ & \$Metal wl \\
\hline 32005 & 5 & 0.00359833 & $(-32005)$ & imp:n=1 & \$Metal wl \\
\hline 32006 & 5 & 0.00359833 & $(-32006)$ & imp:n=1 & \$Metal wl \\
\hline 32007 & 5 & 0.00359833 & $(-32007)$ & imp:n=1 & \$Metal wl \\
\hline 32008 & 5 & 0.00359833 & $(-32008)$ & imp:n=1 & \$Metal wl \\
\hline 32009 & 5 & 0.00359833 & $(-32009)$ & imp:n=1 & \$Metal wl \\
\hline 32010 & 5 & 0.00359833 & $(-32010)$ & imp:n=1 & \$Metal wl \\
\hline 32011 & 5 & 0.00359833 & $(-32011)$ & imp:n=1 & \$Metal wl \\
\hline 32012 & 5 & 0.00359833 & $(-32012)$ & imp:n=1 & \$Metal wl \\
\hline 32013 & 5 & 0.00359833 & $(-32013)$ & imp:n=1 & \$Metal wl \\
\hline 32014 & 5 & 0.00359833 & $(-32014)$ & imp:n=1 & \$Metal wl \\
\hline 32015 & 5 & 0.00359833 & $(-32015)$ & imp:n=1 & \$Metal wl \\
\hline 32016 & 5 & 0.00359833 & $(-32016)$ & imp:n=1 & \$Metal wl \\
\hline c & & & & & \\
\hline & & & & & \\
\hline c Flo & or1 & Room 324/42 & & & \\
\hline 32401 & 1 & -0.001202 & $(-32401)$ & imp:n=1 & \$Air Filled \\
\hline 32402 & 5 & 0.00359833 & $(-324023$ & 401) imp:n= & $=1 \quad$ \$Metal wl \\
\hline 32403 & 1 & -0.001202 & $(-32403)$ & imp:n=1 & \$Air Filled \\
\hline 32404 & 5 & 0.00359833 & $(-324043$ & 403) imp:n= & $=1 \quad$ \$Metal wl \\
\hline 32405 & 5 & 0.00359833 & $(-32405)$ & imp:n=1 & \$Metal wl \\
\hline 32406 & 5 & 0.00359833 & $(-32406)$ & imp:n=1 & \$Metal wl \\
\hline 32407 & 1 & -0.001202 & $(-32407)$ & imp:n=1 & \$Air Filled \\
\hline 32408 & 5 & 0.00359833 & $(-324083$ & 407) imp:n= & $=1 \quad$ \$Metal wl \\
\hline 32409 & 5 & 0.00359833 & $(-32409)$ & imp:n=1 & \$Metal wl \\
\hline 32410 & 5 & 0.00359833 & $(-32410)$ & imp:n=1 & \$Metal wl \\
\hline 32411 & 5 & 0.00359833 & $(-32411)$ & imp:n=1 & \$Metal wl \\
\hline 32412 & 5 & 0.00359833 & $(-32412)$ & imp:n=1 & \$Metal wl \\
\hline 32413 & 5 & 0.00359833 & $(-32413)$ & imp:n=1 & \$Metal wl \\
\hline 32414 & 1 & -0.001202 & $(-32414)$ & imp:n=1 & \$Air Filled \\
\hline 32415 & 5 & 0.00359833 & $(-324153$ & 414) imp:n= & $=1 \quad$ \$Metal wl \\
\hline 32416 & 5 & 0.00359833 & $(-32416)$ & imp:n=1 & \$Metal wl \\
\hline 32417 & 5 & 0.00359833 & $(-32417)$ & imp:n=1 & \$Metal wl \\
\hline C & & & & & \\
\hline & & & & & \\
\hline c Flo & or1 & Room 325/Fi & r Building & & \\
\hline 32501 & 5 & 0.00359833 & $(-32501)$ & imp:n=1 & \$Metal wl \\
\hline 32502 & 5 & 0.00359833 & $(-32502)$ & imp:n=1 & \$Metal wl \\
\hline 32503 & 5 & 0.00359833 & $(-32503)$ & imp:n=1 & \$Metal wl \\
\hline 32504 & 5 & 0.00359833 & $(-32504)$ & imp:n=1 & \$Metal wl \\
\hline 32505 & 5 & 0.00359833 & $(-32505)$ & imp:n=1 & \$Metal wl \\
\hline 32506 & 5 & 0.00359833 & $(-32506)$ & imp:n=1 & \$Metal wl \\
\hline 32507 & 5 & 0.00359833 & $(-32507)$ & imp:n=1 & \$Metal wl \\
\hline 32508 & 5 & 0.00359833 & $(-32508)$ & imp:n=1 & \$Metal wl \\
\hline 32509 & 5 & 0.00359833 & $(-32509)$ & imp:n=1 & \$Metal wl \\
\hline 32510 & 5 & 0.00359833 & $(-32510)$ & imp:n=1 & \$Metal wl \\
\hline 32511 & 5 & 0.00359833 & $(-32511)$ & imp:n=1 & \$Metal wl \\
\hline 32512 & 5 & 0.00359833 & $(-32512)$ & imp:n=1 & \$Metal wl \\
\hline 32513 & 5 & 0.00359833 & $(-32513)$ & imp:n=1 & \$Metal wl \\
\hline 32514 & 5 & 0.00359833 & $(-32514)$ & imp:n=1 & \$Metal wl \\
\hline 32515 & 5 & 0.00359833 & $(-32515)$ & imp:n=1 & \$Metal wl \\
\hline 32516 & 5 & 0.00359833 & $(-32516)$ & imp:n=1 & \$Metal wl \\
\hline 32517 & 5 & 0.00359833 & $(-32517)$ & imp:n=1 & \$Metal wl \\
\hline 32518 & 5 & 0.00359833 & $(-32518)$ & imp:n=1 & \$Metal wl \\
\hline 32519 & 5 & 0.00359833 & $(-32519)$ & imp:n=1 & \$Metal wl \\
\hline 32520 & 5 & 0.00359833 & $(-32520)$ & imp:n=1 & \$Metal wl \\
\hline 32521 & 5 & 0.00359833 & $(-32521)$ & imp:n=1 & \$Metal wl \\
\hline 32522 & 5 & 0.00359833 & $(-32522)$ & imp:n=1 & \$Metal wl \\
\hline
\end{tabular}




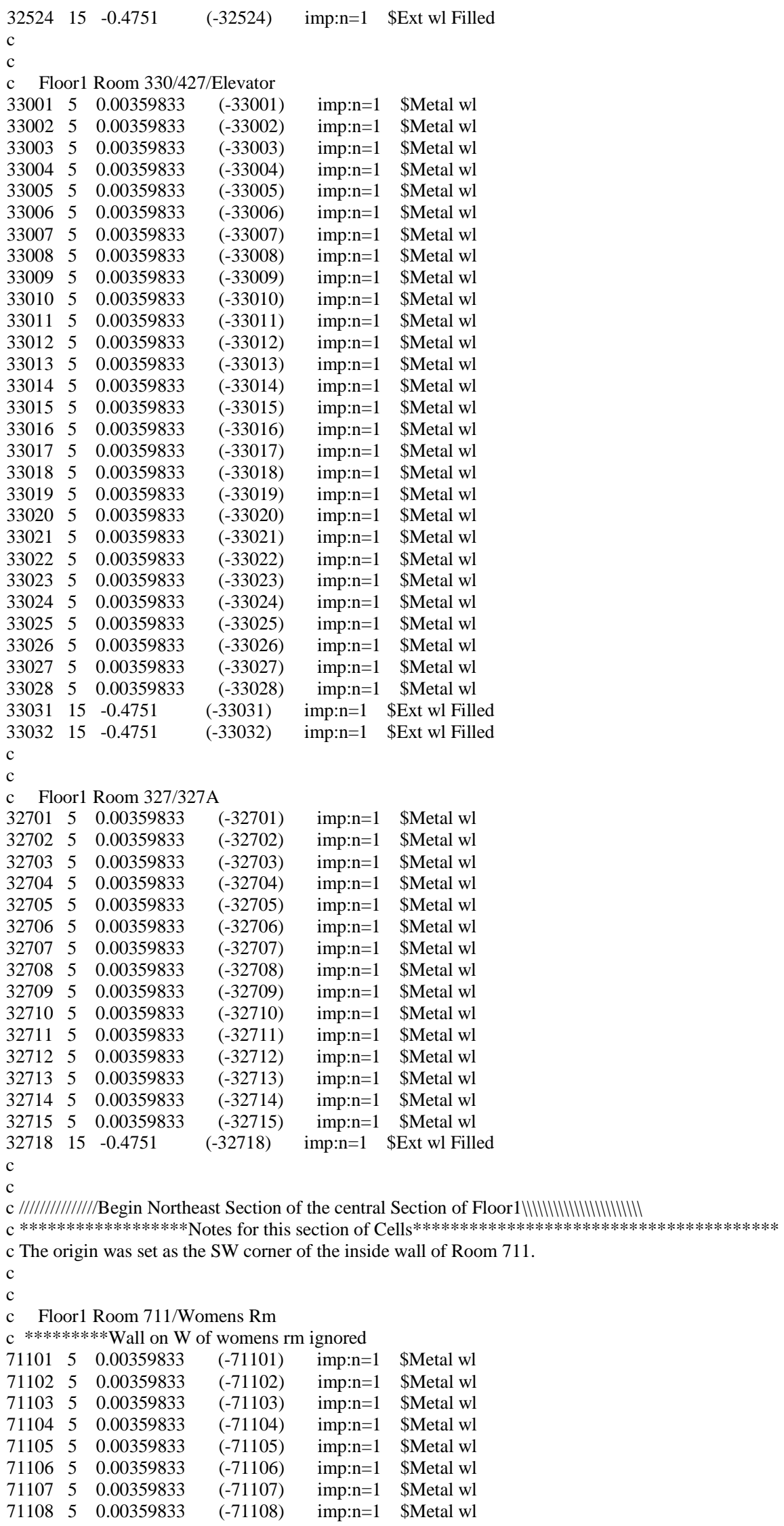




\begin{tabular}{|c|c|c|c|c|c|}
\hline 71109 & 5 & 0.00359833 & $(-71109)$ & imp:n=1 & \$Metal wl \\
\hline 71110 & 5 & 0.00359833 & $(-71110)$ & imp:n=1 & \$Metal wl \\
\hline 71111 & 5 & 0.00359833 & $(-71111)$ & imp:n=1 & \$Metal wl \\
\hline 71112 & 5 & 0.00359833 & $(-71112)$ & imp:n=1 & \$Metal wl \\
\hline 71113 & 5 & 0.00359833 & $(-71113)$ & imp:n=1 & \$Metal wl \\
\hline 71114 & 5 & 0.00359833 & $(-71114)$ & imp:n=1 & \$Metal wl \\
\hline \\
\hline \\
\hline \multicolumn{6}{|c|}{ c $\quad$ Floor1 Room 420} \\
\hline 42001 & 5 & 0.00359833 & $(-42001)$ & imp:n=1 & \$Metal wl \\
\hline 42002 & 5 & 0.00359833 & $(-42002)$ & imp:n=1 & \$Metal wl \\
\hline 42003 & 5 & 0.00359833 & $(-42003)$ & imp:n=1 & \$Metal wl \\
\hline 42004 & 5 & 0.00359833 & $(-42004)$ & imp:n=1 & \$Metal wl \\
\hline 42005 & 5 & 0.00359833 & $(-42005)$ & imp:n=1 & \$Metal wl \\
\hline 42006 & 5 & 0.00359833 & $(-42006)$ & imp:n=1 & \$Metal wl \\
\hline 42007 & 5 & 0.00359833 & $(-42007)$ & imp:n=1 & \$Metal wl \\
\hline 42008 & 5 & 0.00359833 & $(-42008)$ & imp:n=1 & \$Metal wl \\
\hline 42009 & 5 & 0.00359833 & $(-42009)$ & imp:n=1 & \$Metal wl \\
\hline 42010 & 5 & 0.00359833 & $(-42010)$ & imp:n=1 & \$Metal wl \\
\hline 42011 & 5 & 0.00359833 & $(-42011)$ & imp:n=1 & \$Metal wl \\
\hline 42012 & 5 & 0.00359833 & $(-42012)$ & imp:n=1 & \$Metal wl \\
\hline \\
\hline \\
\hline \multicolumn{6}{|c|}{ c Floor1 Room 525/Freezer } \\
\hline 52501 & 5 & 0.00359833 & $(-52501)$ & imp:n=1 & \$Metal wl \\
\hline 52502 & 5 & 0.00359833 & $(-52502)$ & imp:n=1 & \$Metal wl \\
\hline 52503 & 5 & 0.00359833 & $(-52503)$ & imp:n=1 & \$Metal wl \\
\hline 52504 & 5 & 0.00359833 & $(-52504)$ & imp:n=1 & \$Metal wl \\
\hline 52505 & 5 & 0.00359833 & $(-52505)$ & imp:n=1 & \$Metal wl \\
\hline 52506 & 5 & 0.00359833 & $(-52506)$ & imp:n=1 & \$Metal wl \\
\hline 52507 & 5 & 0.00359833 & $(-52507)$ & imp:n=1 & \$Metal wl \\
\hline 52508 & 5 & 0.00359833 & $(-52508)$ & imp:n=1 & \$Metal wl \\
\hline 52509 & 5 & 0.00359833 & $(-52509)$ & imp:n=1 & \$Metal wl \\
\hline 52510 & 5 & 0.00359833 & $(-52510)$ & imp:n=1 & \$Metal wl \\
\hline 52511 & 5 & 0.00359833 & $(-52511)$ & imp:n=1 & \$Metal wl \\
\hline 52512 & 5 & 0.00359833 & $(-52512)$ & imp:n=1 & \\
\hline 52513 & 5 & 0.00359833 & $(-52513)$ & imp:n=1 & \$Metal wl \\
\hline 52514 & 5 & 0.00359833 & $(-52514)$ & imp:n=1 & \$Metal wl \\
\hline 52515 & 5 & 0.00359833 & $(-52515)$ & imp:n=1 & \$Metal wl \\
\hline 52516 & 5 & 0.0035 & $(-52516)$ & imp:n=1 & \$Metal wl \\
\hline 52517 & 5 & 0.00359833 & $(-52517)$ & imp:n=1 & \$Metal wl \\
\hline 52518 & 5 & 0.0035 & $(-52518)$ & imp:n=1 & \$Metal wl \\
\hline 52519 & 5 & 0.00359833 & $(-52519)$ & imp:n=1 & \$Metal wl \\
\hline \\
\hline \\
\hline \multicolumn{6}{|c|}{ c Floor1 Room 426/426A } \\
\hline 42601 & 5 & 0.00359833 & $(-42601)$ & imp:n=1 & \$Metal wl \\
\hline 42602 & 5 & 0.00359833 & $(-42602)$ & imp:n=1 & \$Metal wl \\
\hline 42603 & 5 & 0.00359833 & $(-42603)$ & imp:n=1 & \$Metal wl \\
\hline 42604 & 5 & 0.00359833 & $(-42604)$ & imp:n=1 & \$Metal wl \\
\hline 42605 & 5 & 0.00359833 & $(-42605)$ & imp:n=1 & \$Metal wl \\
\hline 42606 & 5 & 0.00359833 & $(-42606)$ & imp:n=1 & \$Metal wl \\
\hline 42607 & 5 & 0.00359833 & $(-42607)$ & imp:n=1 & \$Metal wl \\
\hline 42608 & 5 & 0.00359833 & $(-42608)$ & imp:n=1 & \$Metal wl \\
\hline 42609 & 5 & 0.00359833 & $(-42609)$ & imp:n=1 & \$Metal wl \\
\hline 42610 & 5 & 0.00359833 & $(-42610)$ & imp:n=1 & \$Metal wl \\
\hline 42611 & 5 & 0.00359833 & $(-42611)$ & imp:n=1 & \$Metal wl \\
\hline 42612 & 5 & 0.00359833 & $(-42612)$ & imp:n=1 & \$Metal wl \\
\hline 42613 & 5 & 0.00359833 & $(-42613)$ & imp:n=1 & \$Metal wl \\
\hline 42614 & 5 & 0.00359833 & $(-42614)$ & imp:n=1 & \$Metal wl \\
\hline 42615 & 5 & 0.00359833 & $(-42615)$ & imp:n=1 & \$Metal wl \\
\hline 42616 & 5 & 0.00359833 & $(-42616)$ & imp:n=1 & \$Metal wl \\
\hline 42617 & 5 & 0.00359833 & $(-42617)$ & imp:n=1 & \$Metal wl \\
\hline 42618 & 5 & 0.00359833 & $(-42618)$ & imp:n=1 & \$Metal wl \\
\hline 42619 & 5 & 0.00359833 & $(-42619)$ & imp:n=1 & \$Metal wl \\
\hline \multicolumn{6}{|c|}{$r$} \\
\hline \\
\hline c Flo & or1 & Room 430/R & eiving Roo & & \\
\hline 43001 & 5 & 0.00359833 & $(-43001)$ & & \\
\hline 43002 & 5 & 0.00359833 & $(-43002)$ & imp:n=1 & \$Metal wl \\
\hline
\end{tabular}




\begin{tabular}{|c|c|c|c|c|}
\hline 430035 & 0.00359833 & $(-43003)$ & imp:n=1 & \$Metal wl \\
\hline 430045 & 0.00359833 & $(-43004)$ & imp:n=1 & \$Metal wl \\
\hline 430055 & 0.00359833 & $(-43005)$ & imp:n=1 & \$Metal wl \\
\hline 430065 & 0.00359833 & $(-43006)$ & imp:n=1 & \$Metal wl \\
\hline 430075 & 0.00359833 & $(-43007)$ & imp:n=1 & \$Metal wl \\
\hline 430085 & 0.00359833 & $(-43008)$ & imp:n=1 & \$Metal wl \\
\hline 430095 & 0.00359833 & $(-43009)$ & imp:n=1 & \$Metal wl \\
\hline 430105 & 0.00359833 & $(-43010)$ & imp:n=1 & \$Metal wl \\
\hline 430115 & 0.00359833 & $(-43011)$ & imp:n=1 & \$Metal wl \\
\hline 430125 & 0.00359833 & $(-43012)$ & imp:n=1 & \$Metal wl \\
\hline 430135 & 0.00359833 & $(-43013)$ & imp:n=1 & \$Metal wl \\
\hline 4301515 & -0.4751 & $(-43015)$ & imp:n=1 & \$Ext wl Filled \\
\hline c & & & & \\
\hline c & & & & \\
\hline Floor & 1 Room 527/5 & $527 \mathrm{~A}$ & & \\
\hline 527015 & 0.00359833 & $(-52701)$ & imp:n=1 & \$Metal wl \\
\hline 527025 & 0.00359833 & $(-52702)$ & imp:n=1 & \$Metal wl \\
\hline 527035 & 0.00359833 & $(-52703)$ & imp:n=1 & \$Metal wl \\
\hline 527045 & 0.00359833 & $(-52704)$ & imp:n=1 & \$Metal wl \\
\hline 527055 & 0.00359833 & $(-52705)$ & imp:n=1 & \$Metal wl \\
\hline 527065 & 0.00359833 & $(-52706)$ & imp:n=1 & \$Metal wl \\
\hline 527075 & 0.00359833 & $(-52707)$ & imp:n=1 & \$Metal wl \\
\hline 527085 & 0.00359833 & $(-52708)$ & imp:n=1 & \$Metal wl \\
\hline 527095 & 0.00359833 & $(-52709)$ & imp:n=1 & \$Metal wl \\
\hline 527105 & 0.00359833 & $(-52710)$ & imp:n=1 & \$Metal wl \\
\hline 527115 & 0.00359833 & $(-52711)$ & imp:n=1 & \$Metal wl \\
\hline 527125 & 0.00359833 & $(-52712)$ & imp:n=1 & \$Metal wl \\
\hline 527135 & 0.00359833 & $(-52713)$ & imp:n=1 & \$Metal wl \\
\hline 527145 & 0.00359833 & $(-52714)$ & imp:n=1 & \$Metal wl \\
\hline 527155 & 0.00359833 & $(-52715)$ & imp:n=1 & \$Metal wl \\
\hline 527165 & 0.00359833 & $(-52716)$ & imp:n=1 & \$Metal wl \\
\hline 527175 & 0.00359833 & $(-52717)$ & imp:n=1 & \$Metal wl \\
\hline 527185 & 0.00359833 & $(-52718)$ & imp:n=1 & \$Metal wl \\
\hline 527195 & 0.00359833 & $(-52719)$ & imp:n=1 & \$Metal wl \\
\hline 527205 & 0.00359833 & $(-52720)$ & imp:n=1 & \$Metal wl \\
\hline 527215 & 0.00359833 & $(-52721)$ & imp:n=1 & \$Metal wl \\
\hline 527225 & 0.00359833 & $(-52722)$ & imp:n=1 & \$Metal wl \\
\hline 527235 & 0.00359833 & $(-52723)$ & imp:n=1 & \$Metal wl \\
\hline 5272615 & -0.4751 & $(-52726)$ & imp:n=1 & \$Ext wl Filled \\
\hline c & & & & \\
\hline c & & & & \\
\hline c Floor & 1 Room 529 & & & \\
\hline 529015 & 0.00359833 & $(-52901)$ & imp:n=1 & \$Metal wl \\
\hline 529025 & 0.00359833 & $(-52902)$ & imp:n=1 & \$Metal wl \\
\hline 529035 & 0.00359833 & $(-52903)$ & imp:n=1 & \$Metal wl \\
\hline 529045 & 0.00359833 & $(-52904)$ & imp:n=1 & \$Metal wl \\
\hline 529055 & 0.00359833 & $(-52905)$ & imp:n=1 & \$Metal wl \\
\hline 529065 & 0.00359833 & $(-52906)$ & imp:n=1 & \$Metal wl \\
\hline 529075 & 0.00359833 & $(-52907)$ & imp:n=1 & \$Metal wl \\
\hline 529085 & 0.00359833 & $(-52908)$ & imp:n=1 & \$Metal wl \\
\hline 529095 & 0.00359833 & $(-52909)$ & imp:n=1 & \$Metal wl \\
\hline 529105 & 0.00359833 & $(-52910)$ & imp:n=1 & \$Metal wl \\
\hline 529115 & 0.00359833 & $(-52911)$ & imp:n=1 & \$Metal wl \\
\hline 529125 & 0.00359833 & $(-52912)$ & imp:n=1 & \$Metal wl \\
\hline 529135 & 0.00359833 & $(-52913)$ & imp:n=1 & \$Metal wl \\
\hline 529143 & -2.3 & $(-52914)$ & imp:n=1 $\quad \$ C$ & Conc. Filled \\
\hline 529153 & -2.3 & $(-52915)$ & imp:n=1 & Conc. Filled \\
\hline c & & & & \\
\hline c & & & & \\
\hline c Floor & 1 Room 520 & & & \\
\hline 520015 & 0.00359833 & $(-52001)$ & imp:n=1 & \$Metal wl \\
\hline 520025 & 0.00359833 & $(-52002)$ & imp:n=1 & \$Metal wl \\
\hline 520035 & 0.00359833 & $(-52003)$ & imp:n=1 & \$Metal wl \\
\hline 520045 & 0.00359833 & $(-52004)$ & imp:n=1 & \$Metal wl \\
\hline 520055 & 0.00359833 & $(-52005)$ & imp:n=1 & \$Metal wl \\
\hline 520065 & 0.00359833 & $(-52006)$ & imp:n=1 & \$Metal wl \\
\hline 520075 & 0.00359833 & $(-52007)$ & imp:n=1 & \$Metal wl \\
\hline 520085 & 0.00359833 & $(-52008)$ & imp:n=1 & \$Metal wl \\
\hline 520095 & 0.00359833 & $(-52009)$ & imp:n=1 & \$Metal wl \\
\hline 520105 & 0.00359833 & $(-52010)$ & imp:n=1 & \$Metal wl \\
\hline
\end{tabular}




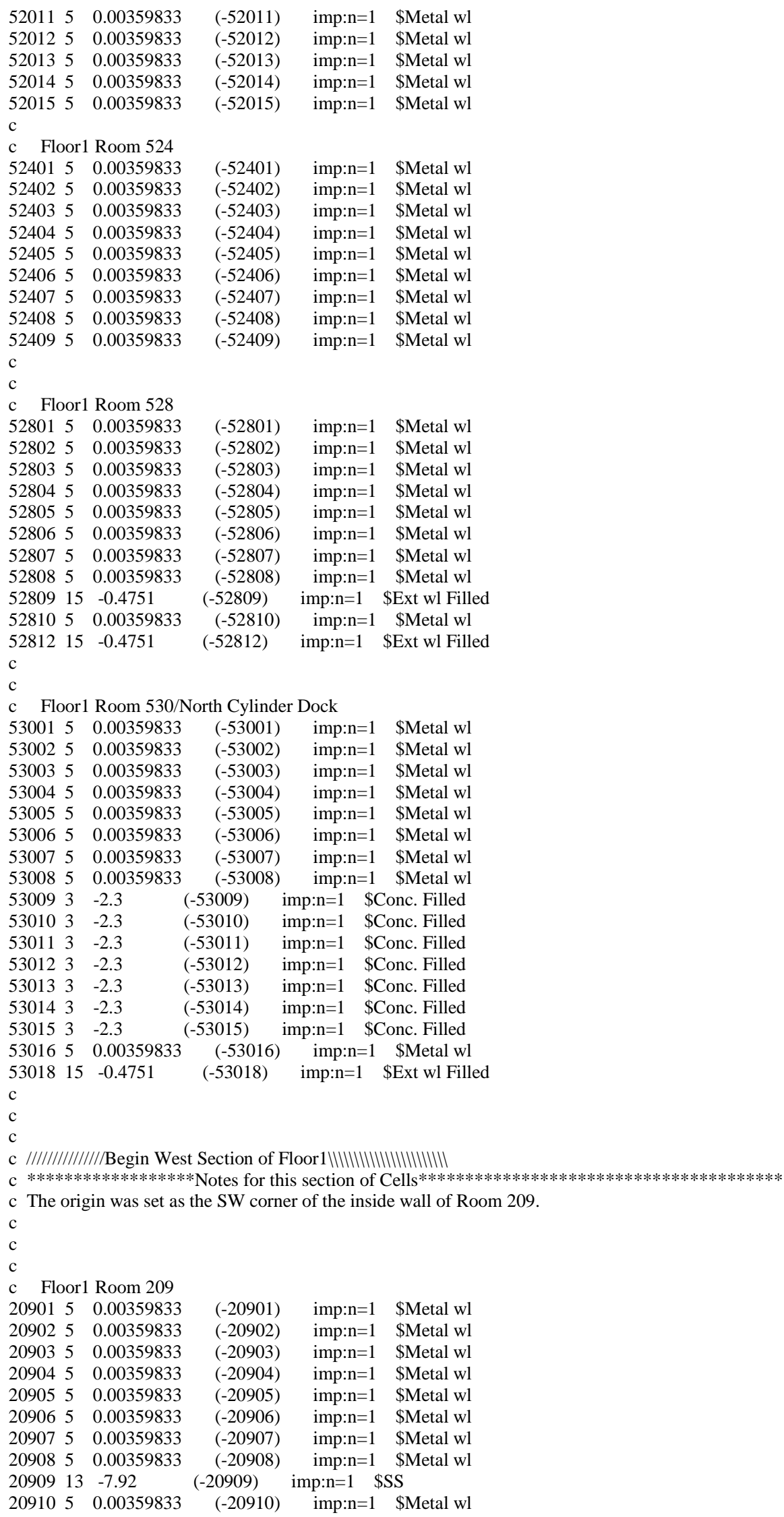




\begin{tabular}{|c|c|c|c|}
\hline & & \multirow[b]{2}{*}{$(-20911)$} & \multirow[b]{2}{*}{ imp:n=1 \$Metal wl } \\
\hline 209115 & 0.00359833 & & \\
\hline 209125 & 0.00359833 & $(-20912)$ & imp:n=1 \$Metal wl \\
\hline 209135 & 0.00359833 & $(-20913)$ & \$Metal wl \\
\hline 209145 & 0.00359833 & $(-20914)$ & \$Metal wl \\
\hline 209155 & 0.00359833 & $(-20915)$ & \$Metal wl \\
\hline 209165 & 0.00359833 & $(-20916)$ & \$Metal wl \\
\hline 209175 & 0.00359833 & $(-20917)$ & \$Metal wl \\
\hline 209185 & 0.00359833 & $(-20918)$ & \$Metal wl \\
\hline 209205 & 0.00359833 & $(-20920)$ & imp:n=1 \$Metal wl \\
\hline 2092115 & -0.4751 & $(-20921)$ & imp:n=1 \$Ext wl Filled \\
\hline 2092215 & -0.4751 & $(-20922)$ & \$Ext wl Filled \\
\hline \multicolumn{4}{|l|}{ c } \\
\hline \\
\hline \multicolumn{4}{|c|}{ c Floor1 Room 202/203 } \\
\hline 202015 & 0.00359833 & $(-20201)$ & \$Metal wl \\
\hline 202025 & 0.00359833 & $(-20202)$ & \$Metal wl \\
\hline 2020315 & -0.4751 & $(-20203)$ & imp:n=1 \$Ext wl Filled \\
\hline 202045 & 0.00359833 & $(-20204)$ & imp:n=1 \$Metal wl \\
\hline 2020515 & -0.4751 & $(-20205)$ & imp:n=1 \$Ext wl Filled \\
\hline 202065 & 0.00359833 & $(-20206)$ & imp:n=1 \$Metal wl \\
\hline 202075 & 0.00359833 & $(-20207)$ & \$Metal wl \\
\hline 202085 & 0.00359833 & $(-20208)$ & \$Metal wl \\
\hline 202095 & 0.00359833 & $(-20209)$ & \$Metal wl \\
\hline 202105 & 0.00359833 & $(-20210)$ & \$Metal wl \\
\hline 202115 & 0.00359833 & $(-20211)$ & \$Metal wl \\
\hline 202125 & 0.00359833 & $(-20212)$ & \$Metal wl \\
\hline 202135 & 0.00359833 & $(-20213)$ & \$Metal wl \\
\hline 202145 & 0.00359833 & $(-20214)$ & \$Metal wl \\
\hline 202155 & 0.00359833 & $(-20215)$ & \$Metal wl \\
\hline 202165 & 0.00359833 & $(-20216)$ & \$Metal wl \\
\hline 202175 & 0.00359833 & $(-20217)$ & \$Metal wl \\
\hline 202185 & 0.00359833 & $(-20218)$ & \$Metal wl \\
\hline 202195 & 0.00359833 & $(-20219)$ & imp:n=1 \$Metal wl \\
\hline 2022015 & -0.4751 & $(-20220)$ & imp:n=1 \$Ext wl Filled \\
\hline \multicolumn{4}{|c|}{$\mathrm{C}$} \\
\hline \multicolumn{4}{|l|}{ c } \\
\hline \multicolumn{4}{|c|}{ c $\quad$ Floor1 Room 200} \\
\hline 200015 & 0.00359833 & $(-20001)$ & \$Metal wl \\
\hline 200025 & 0.00359833 & $(-20002)$ & \$Metal wl \\
\hline 200035 & 0.00359833 & $(-20003)$ & \$Metal wl \\
\hline 200045 & 0.00359833 & $(-20004)$ & \$Metal wl \\
\hline 200055 & 0.00359833 & $(-20005)$ & \$Metal wl \\
\hline 200065 & 0.00359833 & $(-20006)$ & \$Metal wl \\
\hline 200075 & 0.00359833 & $(-20007)$ & \$Metal wl \\
\hline 200085 & 0.00359833 & $(-20008)$ & \$Metal wl \\
\hline 200095 & 0.00359833 & $(-20009)$ & \$Metal wl \\
\hline 200105 & 0.00359833 & $(-20010)$ & \$Metal wl \\
\hline 200115 & 0.00359833 & $(-20011)$ & \$Metal wl \\
\hline 200125 & 0.00359833 & $(-20012)$ & \$Metal wl \\
\hline 200135 & 0.00359833 & $(-20013)$ & \$Metal wl \\
\hline 200145 & 0.00359833 & $(-20014)$ & \$Metal wl \\
\hline \multicolumn{4}{|l|}{ c } \\
\hline \multicolumn{4}{|c|}{ 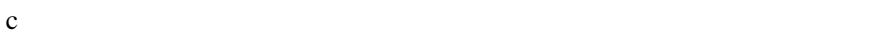 } \\
\hline c Floor1 & 1 Room 201 & & \\
\hline $\mathrm{c} * * * * * * *$ & $* * * * * * * * * * \mathrm{~T}$ & his room con & tains the SAL hot cellsL $* * * * * * * * * * * * * *$ \\
\hline c Neglect & ed thin wall st & trip in NE bor & rder region \\
\hline 2010113 & -7.92 & $(-20101)$ & imp:n=1 \$SS \\
\hline 201025 & 0.00359833 & $(-20102)$ & imp:n=1 \$Metal wl \\
\hline 2010313 & -7.92 & $(-20103)$ & imp:n=1 \$SS \\
\hline 2010413 & -7.92 & $(-20104)$ & imp:n=1 \$SS \\
\hline 201055 & 0.00359833 & $(-20105)$ & imp:n=1 \$Metal wl \\
\hline 201065 & 0.00359833 & $(-20106)$ & \$Metal wl \\
\hline 201075 & 0.00359833 & $(-20107)$ & \$Metal wl \\
\hline 201085 & 0.00359833 & $(-20108)$ & \$Metal wl \\
\hline 201095 & 0.00359833 & $(-20109)$ & \$Metal wl \\
\hline 201105 & 0.00359833 & $(-20110)$ & \$Metal wl \\
\hline 201115 & 0.00359833 & $(-20111)$ & imp:n=1 \$Metal wl \\
\hline 2011215 & -0.4751 & $(-20112)$ & imp:n=1 \$Ext wl Filled \\
\hline 201135 & 0.00359833 & $(-20113)$ & imp:n=1 \$Metal wl \\
\hline 201145 & 0.00359833 & $(-20114)$ & \$Metal wl \\
\hline
\end{tabular}




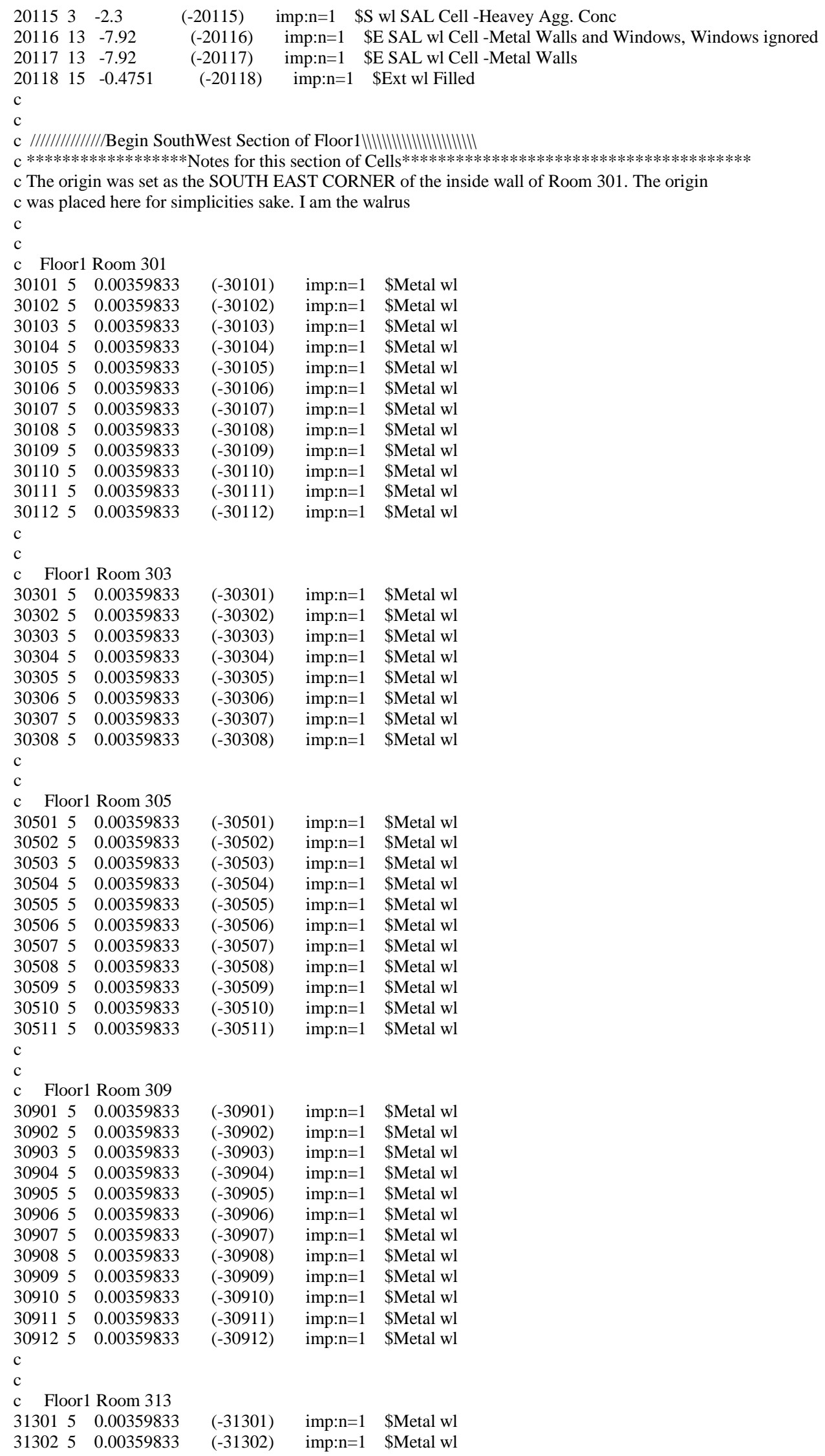

(-30301)

$(-30302)$

$(-30303)$

$(-30304)$

$(-30305)$

$(-30306)$

$(-30307)$

(-30308)

$(-30501)$

$(-30502)$

$(-30503)$

$(-30504)$

$(-30505)$

(-30506)

$(-30507)$

$(-30508)$

$(-30509)$

$(-30510)$

(-30511)

(-30901)

$(-30902)$

$(-30903)$

$(-30904)$

$(-30905)$

$(-30906)$

$(-30907)$

$(-30908)$

(-30909)

(-30910)

(-30911)

(-30912)

imp:n=1 \$Metal wl imp:n=1 \$Metal wl imp:n=1 \$Metal wl imp:n=1 \$Metal wl imp:n=1 \$Metal wl imp:n=1 \$Metal wl imp:n=1 \$Metal wl imp:n=1 \$Metal wl

imp:n=1 \$Metal wl imp:n=1 \$Metal wl imp:n=1 \$Metal wl imp:n=1 \$Metal wl imp:n=1 \$Metal wl imp:n=1 \$Metal wl imp:n=1 \$Metal wl imp:n=1 \$Metal wl imp:n=1 \$Metal wl imp:n=1 \$Metal wl imp:n=1 \$Metal wl 


\begin{tabular}{|c|c|c|c|c|}
\hline 313035 & 0.00359833 & $(-31303)$ & imp:n=1 & \$Metal wl \\
\hline 313045 & 0.00359833 & $(-31304)$ & imp:n=1 & \$Metal wl \\
\hline 313055 & 0.00359833 & $(-31305)$ & imp:n=1 & \$Metal wl \\
\hline 313065 & 0.00359833 & $(-31306)$ & imp:n=1 & \$Metal wl \\
\hline 313075 & 0.00359833 & $(-31307)$ & imp:n=1 & \$Metal wl \\
\hline 313085 & 0.00359833 & $(-31308)$ & imp:n=1 & \$Metal wl \\
\hline 313095 & 0.00359833 & $(-31309)$ & imp:n=1 & \$Metal wl \\
\hline 313105 & 0.00359833 & $(-31310)$ & imp:n=1 & \$Metal wl \\
\hline 313115 & 0.00359833 & $(-31311)$ & imp:n=1 & \$Metal wl \\
\hline 313125 & 0.00359833 & $(-31312)$ & imp:n=1 & \$Metal wl \\
\hline \\
\hline \multirow{2}{*}{\multicolumn{5}{|c|}{1 Room 317}} \\
\hline & & & & \\
\hline 317015 & 0.00359833 & $(-31701)$ & imp:n=1 & \$Metal wl \\
\hline 317025 & 0.00359833 & $(-31702)$ & imp:n=1 & \$Metal wl \\
\hline 317035 & 0.00359833 & $(-31703)$ & imp:n=1 & \$Metal wl \\
\hline \multicolumn{5}{|c|}{ 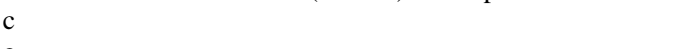 } \\
\hline & & & & \\
\hline \multicolumn{5}{|c|}{ с $\quad$ Floor1 Room 319/319A } \\
\hline 319015 & 0.00359833 & $(-31901)$ & imp:n=1 & \$Metal wl \\
\hline 319025 & 0.00359833 & $(-31902)$ & imp:n=1 & \$Metal wl \\
\hline 319035 & 0.00359833 & $(-31903)$ & imp:n=1 & \$Metal wl \\
\hline 319045 & 0.00359833 & $(-31904)$ & imp:n=1 & \$Metal wl \\
\hline 319055 & 0.00359833 & $(-31905)$ & imp:n=1 & \$Metal wl \\
\hline 319065 & 0.00359833 & $(-31906)$ & imp:n=1 & \$Metal wl \\
\hline 319075 & 0.00359833 & $(-31907)$ & imp:n=1 & \$Metal wl \\
\hline 319085 & 0.00359833 & $(-31908)$ & imp:n=1 & \$Metal wl \\
\hline 319095 & 0.00359833 & $(-31909)$ & imp:n=1 & \$Metal wl \\
\hline 319105 & 0.00359833 & $(-31910)$ & imp:n=1 & \$Metal wl \\
\hline 319115 & 0.00359833 & $(-31911)$ & imp:n=1 & \$Metal wl \\
\hline \\
\hline & & & & \\
\hline \multicolumn{5}{|c|}{ c Floor1 Room 300} \\
\hline 300015 & 0.00359833 & $(-30001)$ & imp:n=1 & \$Metal wl \\
\hline 300025 & 0.00359833 & $(-30002)$ & imp:n=1 & \$Metal wl \\
\hline 300035 & 0.00359833 & $(-30003)$ & imp:n=1 & \$Metal wl \\
\hline 300045 & 0.00359833 & $(-30004)$ & imp:n=1 & \$Metal wl \\
\hline 300055 & 0.00359833 & $(-30005)$ & imp:n=1 & \$Metal wl \\
\hline 300065 & 0.00359833 & $(-30006)$ & imp:n=1 & \$Metal wl \\
\hline 300075 & 0.00359833 & $(-30007)$ & imp:n=1 & \$Metal wl \\
\hline 300085 & 0.00359833 & $(-30008)$ & imp:n=1 & \$Metal wl \\
\hline 300095 & 0.00359833 & $(-30009)$ & imp:n=1 & \$Metal wl \\
\hline 300105 & 0.00359833 & $(-30010)$ & imp:n=1 & \$Metal wl \\
\hline 300115 & 0.00359833 & $(-30011)$ & imp:n=1 & \$Metal wl \\
\hline 300125 & 0.00359833 & $(-30012)$ & imp:n=1 & \$Metal wl \\
\hline 300135 & 0.00359833 & $(-30013)$ & imp:n=1 & \$Metal wl \\
\hline 300145 & 0.00359833 & $(-30014)$ & imp:n=1 & \$Metal wl \\
\hline 300155 & 0.00359833 & $(-30015)$ & imp:n=1 & \$Metal wl \\
\hline 300165 & 0.00359833 & $(-30016)$ & imp:n=1 & \$Metal wl \\
\hline 300175 & 0.00359833 & $(-30017)$ & imp:n=1 & \$Metal wl \\
\hline 300185 & 0.00359833 & $(-30018)$ & imp:n=1 & \$Metal wl \\
\hline 300195 & 0.00359833 & $(-30019)$ & imp:n=1 & \$Metal wl \\
\hline 300205 & 0.00359833 & $(-30020)$ & imp:n=1 & \$Metal wl \\
\hline 300215 & 0.00359833 & $(-30021)$ & imp:n=1 & \$Metal wl \\
\hline 300225 & 0.00359833 & $(-30022)$ & imp:n=1 & \$Metal wl \\
\hline 300235 & 0.00359833 & $(-30023)$ & imp:n=1 & \$Metal wl \\
\hline \\
\hline \\
\hline & & & & \\
\hline \multicolumn{5}{|c|}{ c $\quad$ Floor1 Room 306} \\
\hline 306015 & 0.00359833 & $(-30601)$ & imp:n=1 & \$Metal wl \\
\hline 306025 & 0.00359833 & $(-30602)$ & imp:n=1 & \$Metal wl \\
\hline 306035 & 0.00359833 & $(-30603)$ & imp:n=1 & \$Metal wl \\
\hline 306045 & 0.00359833 & $(-30604)$ & imp:n=1 & \$Metal wl \\
\hline 306055 & 0.00359833 & $(-30605)$ & imp:n=1 & \$Metal wl \\
\hline 306065 & 0.00359833 & $(-30606)$ & imp:n=1 & \$Metal wl \\
\hline 306075 & 0.00359833 & $(-30607)$ & imp:n=1 & \$Metal wl \\
\hline 306085 & 0.00359833 & $(-30608)$ & imp:n=1 & \$Metal wl \\
\hline 306095 & 0.00359833 & $(-30609)$ & imp:n=1 & \$Metal wl \\
\hline 306105 & 0.00359833 & $(-30610)$ & imp:n=1 & \$Metal wl \\
\hline
\end{tabular}




\begin{tabular}{|c|c|c|c|c|}
\hline $\begin{array}{l}306115 \\
\text { c }\end{array}$ & 0.00359833 & $(-30611)$ & imp:n=1 & \$Metal wl \\
\hline & & & & \\
\hline Floor & 1 Room 310 & & & \\
\hline 310015 & 0.00359833 & $(-31001)$ & imp:n=1 & \$Metal wl \\
\hline 310025 & 0.00359833 & $(-31002)$ & imp:n=1 & \$Metal wl \\
\hline 310035 & 0.00359833 & $(-31003)$ & imp:n=1 & \$Metal wl \\
\hline 310045 & 0.00359833 & $(-31004)$ & imp:n=1 & \$Metal wl \\
\hline 310055 & 0.00359833 & $(-31005)$ & imp:n=1 & \$Metal wl \\
\hline c & & & & \\
\hline & & & & \\
\hline c Floor- & 1 Room 312 & & & \\
\hline 312015 & 0.00359833 & $(-31201)$ & imp:n=1 & \$Metal wl \\
\hline 312025 & 0.00359833 & $(-31202)$ & imp:n=1 & \$Metal wl \\
\hline 312035 & 0.00359833 & $(-31203)$ & imp:n=1 & \$Metal wl \\
\hline 312045 & 0.00359833 & $(-31204)$ & imp:n=1 & \$Metal wl \\
\hline 312055 & 0.00359833 & $(-31205)$ & imp:n=1 & \$Metal wl \\
\hline 312065 & 0.00359833 & $(-31206)$ & imp:n=1 & \$Metal wl \\
\hline 312075 & 0.00359833 & $(-31207)$ & imp:n=1 & \$Metal wl \\
\hline 312085 & 0.00359833 & $(-31208)$ & imp:n=1 & \$Metal wl \\
\hline 312095 & 0.00359833 & $(-31209)$ & imp:n=1 & \$Metal wl \\
\hline 312105 & 0.00359833 & $(-31210)$ & imp:n=1 & \$Metal wl \\
\hline 312115 & 0.00359833 & $(-31211)$ & imp:n=1 & \$Metal wl \\
\hline 312125 & 0.00359833 & $(-31212)$ & imp:n=1 & \$Metal wl \\
\hline 312135 & 0.00359833 & $(-31213)$ & imp:n=1 & \$Metal wl \\
\hline 312145 & 0.00359833 & $(-31214)$ & imp:n=1 & \$Metal wl \\
\hline 312155 & 0.00359833 & $(-31215)$ & imp:n=1 & \$Metal wl \\
\hline 312165 & 0.00359833 & $(-31216)$ & imp:n=1 & \$Metal wl \\
\hline c & & & & \\
\hline c & & & & \\
\hline Floor & 1 Room 316 & & & \\
\hline 316015 & 0.00359833 & $(-31601)$ & imp:n=1 & \$Metal wl \\
\hline 3160213 & -7.92 & $(-31602)$ & imp: $n=1 \quad \$$ & \$SS \\
\hline 316035 & 0.00359833 & $(-31603)$ & imp:n=1 & \$Metal wl \\
\hline 316045 & 0.00359833 & $(-31604)$ & imp:n=1 & \$Metal wl \\
\hline 316055 & 0.00359833 & $(-31605)$ & imp:n=1 & \$Metal wl \\
\hline 316065 & 0.00359833 & $(-31606)$ & imp:n=1 & \$Metal wl \\
\hline 316075 & 0.00359833 & $(-31607)$ & imp:n=1 & \$Metal wl \\
\hline 316085 & 0.00359833 & $(-31608)$ & imp:n=1 & \$Metal wl \\
\hline 316095 & 0.00359833 & $(-31609)$ & imp:n=1 & \$Metal wl \\
\hline 316105 & 0.00359833 & $(-31610)$ & imp:n=1 & \$Metal wl \\
\hline 316115 & 0.00359833 & $(-31611)$ & imp:n=1 & \$Metal wl \\
\hline C & & & & \\
\hline & & & & \\
\hline c Floor & 1 Room 401/4 & & & \\
\hline 401015 & 0.00359833 & $(-40101)$ & imp:n=1 & \$Metal wl \\
\hline 401025 & 0.00359833 & $(-40102)$ & imp:n=1 & \$Metal wl \\
\hline 401035 & 0.00359833 & $(-40103)$ & imp:n=1 & \$Metal wl \\
\hline 401045 & 0.00359833 & $(-40104)$ & imp:n=1 & \$Metal wl \\
\hline 401055 & 0.00359833 & $(-40105)$ & imp:n=1 & \$Metal wl \\
\hline 401065 & 0.00359833 & $(-40106)$ & imp:n=1 & \$Metal wl \\
\hline 401075 & 0.00359833 & $(-40107)$ & imp:n=1 & \$Metal wl \\
\hline 401085 & 0.00359833 & $(-40108)$ & imp:n=1 & \$Metal wl \\
\hline 401095 & 0.00359833 & $(-40109)$ & imp:n=1 & \$Metal wl \\
\hline 401105 & 0.00359833 & $(-40110)$ & imp:n=1 & \$Metal wl \\
\hline 401115 & 0.00359833 & $(-40111)$ & imp:n=1 & \$Metal wl \\
\hline 401125 & 0.00359833 & $(-40112)$ & imp:n=1 & \$Metal wl \\
\hline 401135 & 0.00359833 & $(-40113)$ & imp:n=1 & \$Metal wl \\
\hline 401145 & 0.00359833 & $(-40114)$ & imp:n=1 & \$Metal wl \\
\hline 401155 & 0.00359833 & $(-40115)$ & imp:n=1 & \$Metal wl \\
\hline c & & & & \\
\hline c & & & & \\
\hline c Floor & 1 Room 405 & & & \\
\hline 405015 & 0.00359833 & $(-40501)$ & imp:n=1 & \$Metal wl \\
\hline 405025 & 0.00359833 & $(-40502)$ & imp:n=1 & \$Metal wl \\
\hline 405035 & 0.00359833 & $(-40503)$ & imp:n=1 & \$Metal wl \\
\hline 405045 & 0.00359833 & $(-40504)$ & imp:n=1 & \$Metal wl \\
\hline 405055 & 0.00359833 & $(-40505)$ & imp:n=1 & \$Metal wl \\
\hline 405065 & 0.00359833 & $(-40506)$ & imp:n=1 & \$Metal wl \\
\hline 405075 & 0.00359833 & $(-40507)$ & imp:n=1 & \$Metal wl \\
\hline
\end{tabular}




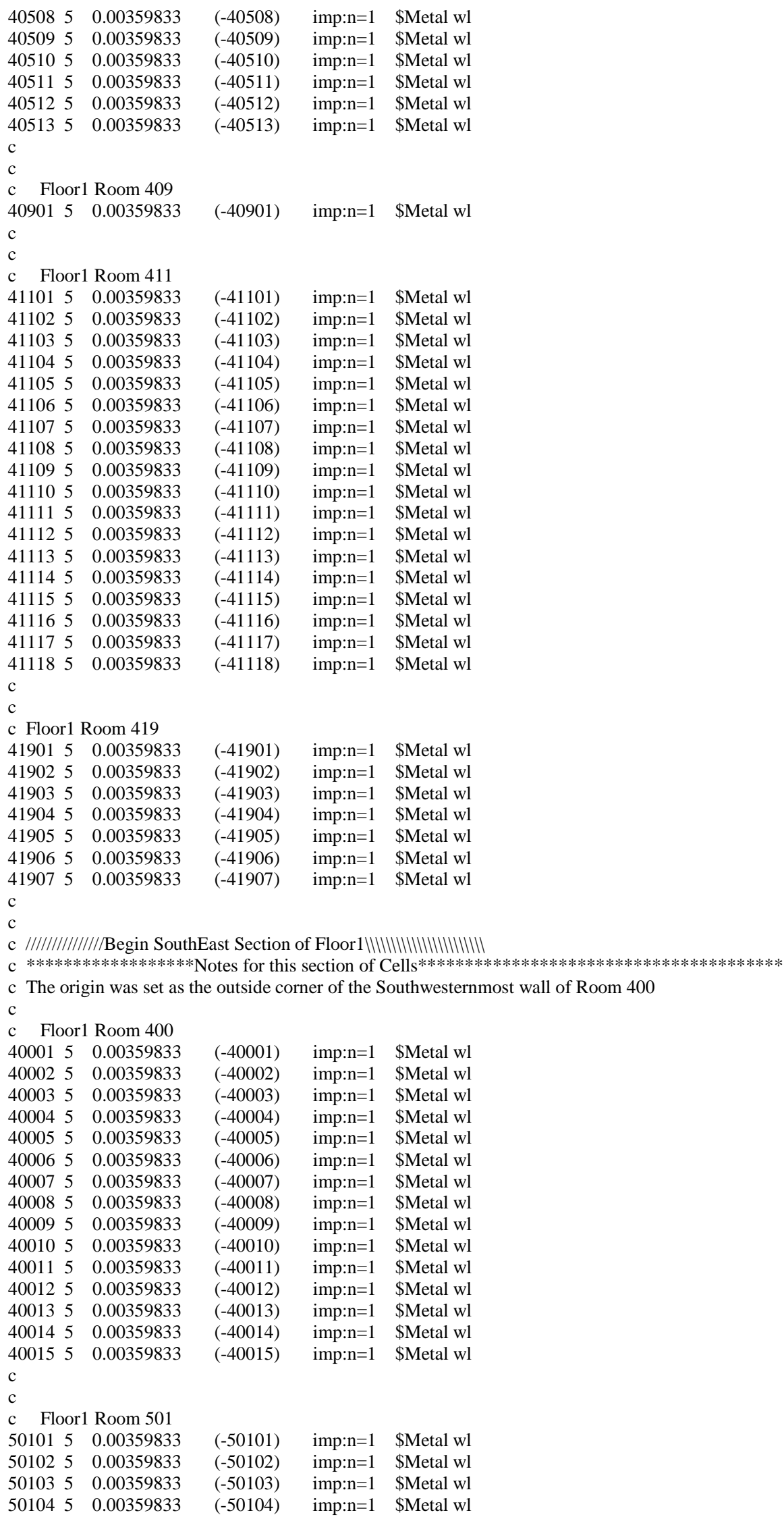




\begin{tabular}{|c|c|c|c|c|}
\hline 501055 & 0.00359833 & $(-50105)$ & imp:n=1 & \$Metal wl \\
\hline 501065 & 0.00359833 & $(-50106)$ & imp:n=1 & \$Metal wl \\
\hline 501075 & 0.00359833 & $(-50107)$ & imp:n=1 & \$Metal wl \\
\hline 501085 & 0.00359833 & $(-50108)$ & imp:n=1 & \$Metal wl \\
\hline 501095 & 0.00359833 & $(-50109)$ & imp: $n=1$ & \$Metal wl \\
\hline 501105 & 0.00359833 & $(-50110)$ & imp: $n=1$ & \$Metal wl \\
\hline 501115 & 0.00359833 & $(-50111)$ & imp:n=1 & \$Metal wl \\
\hline \\
\hline \\
\hline \multicolumn{5}{|c|}{ с $\quad$ Floor1 Room 500} \\
\hline 500015 & 0.00359833 & $(-50001)$ & imp:n=1 & \$Metal wl \\
\hline 500025 & 0.00359833 & $(-50002)$ & imp:n=1 & \$Metal wl \\
\hline 500035 & 0.00359833 & $(-50003)$ & imp:n=1 & \$Metal wl \\
\hline 500045 & 0.00359833 & $(-50004)$ & imp:n=1 & \$Metal wl \\
\hline 500055 & 0.00359833 & $(-50005)$ & imp:n=1 & \$Metal wl \\
\hline 500065 & 0.00359833 & $(-50006)$ & imp:n=1 & \$Metal wl \\
\hline 500075 & 0.00359833 & $(-50007)$ & imp:n=1 & \$Metal wl \\
\hline 500085 & 0.00359833 & $(-50008)$ & imp:n=1 & \$Metal wl \\
\hline 500095 & 0.00359833 & $(-50009)$ & imp:n=1 & \$Metal wl \\
\hline 500105 & 0.00359833 & $(-50010)$ & imp:n=1 & \$Metal wl \\
\hline 500115 & 0.00359833 & $(-50011)$ & imp:n=1 & \$Metal wl \\
\hline 500125 & 0.00359833 & $(-50012)$ & imp:n=1 & \$Metal wl \\
\hline 500135 & 0.00359833 & $(-50013)$ & imp:n=1 & \$Metal wl \\
\hline 500145 & 0.00359833 & $(-50014)$ & imp:n=1 & \$Metal wl \\
\hline 500155 & 0.00359833 & $(-50015)$ & imp:n=1 & \$Metal wl \\
\hline 500165 & 0.00359833 & $(-50016)$ & imp:n=1 & \$Metal wl \\
\hline \multicolumn{5}{|l|}{ c } \\
\hline \multicolumn{5}{|l|}{ c } \\
\hline \multirow{2}{*}{\multicolumn{5}{|c|}{ Floor1 Room 404}} \\
\hline c Floor & 1 Room 404 & & & \\
\hline 404015 & 0.00359833 & $(-40401)$ & imp:n=1 & \$Metal wl \\
\hline 404025 & 0.00359833 & $(-40402)$ & imp:n=1 & \$Metal wl \\
\hline 404035 & 0.00359833 & $(-40403)$ & imp:n=1 & \$Metal wl \\
\hline 404045 & 0.00359833 & $(-40404)$ & imp:n=1 & \$Metal wl \\
\hline \multirow{2}{*}{\multicolumn{5}{|c|}{$\begin{array}{l}\text { c } \\
c\end{array}$}} \\
\hline & & & & \\
\hline \multicolumn{5}{|c|}{ с Floor1 Room 505} \\
\hline 5050113 & -7.92 & $(-50501)$ & imp:n=1 & SS \\
\hline 505025 & 0.00359833 & $(-50502)$ & imp:n=1 & \$Metal wl \\
\hline 505035 & 0.00359833 & $(-50503)$ & imp:n=1 & \$Metal wl \\
\hline 505045 & 0.00359833 & $(-50504)$ & imp:n=1 & \$Metal wl \\
\hline 505055 & 0.00359833 & $(-50505)$ & imp:n=1 & \$Metal wl \\
\hline 505065 & 0.00359833 & $(-50506)$ & imp:n=1 & \$Metal wl \\
\hline \multicolumn{5}{|l|}{ c } \\
\hline \multicolumn{5}{|l|}{ c } \\
\hline \multicolumn{5}{|c|}{ c Floor1 Room 504/504A } \\
\hline 504015 & 0.00359833 & $(-50401)$ & imp:n=1 & \$Metal wl \\
\hline 504025 & 0.00359833 & $(-50402)$ & imp:n=1 & \$Metal wl \\
\hline 504035 & 0.00359833 & $(-50403)$ & imp:n=1 & \$Metal wl \\
\hline 504045 & 0.00359833 & $(-50404)$ & imp:n=1 & \$Metal wl \\
\hline 504055 & 0.00359833 & $(-50405)$ & imp:n=1 & \$Metal wl \\
\hline \multicolumn{5}{|l|}{ c } \\
\hline c & & & & \\
\hline c Floor & 1 Room 406 & & & \\
\hline 4060113 & -7.92 & $(-40601)$ & imp:n=1 & SS \\
\hline 406025 & 0.00359833 & $(-40602)$ & imp:n=1 & \$Metal wl \\
\hline 406035 & 0.00359833 & $(-40603)$ & imp:n=1 & \$Metal wl \\
\hline 406045 & 0.00359833 & $(-40604)$ & imp:n=1 & \$Metal wl \\
\hline 406055 & 0.00359833 & $(-40605)$ & imp:n=1 & \$Metal wl \\
\hline 406065 & 0.00359833 & $(-40606)$ & imp:n=1 & \$Metal wl \\
\hline 406075 & 0.00359833 & $(-40607)$ & imp:n=1 & \$Metal wl \\
\hline 406085 & 0.00359833 & $(-40608)$ & imp:n=1 & \$Metal wl \\
\hline 406095 & 0.00359833 & $(-40609)$ & imp:n=1 & \$Metal wl \\
\hline 406105 & 0.00359833 & $(-40610)$ & imp:n=1 & \$Metal wl \\
\hline 406115 & 0.00359833 & $(-40611)$ & imp:n=1 & \$Metal wl \\
\hline 406125 & 0.00359833 & $(-40612)$ & imp:n=1 & \$Metal wl \\
\hline 406135 & 0.00359833 & $(-40613)$ & imp:n=1 & \$Metal wl \\
\hline 406145 & 0.00359833 & $(-40614)$ & imp:n=1 & \$Metal wl \\
\hline c & & & & \\
\hline & & & & \\
\hline
\end{tabular}




\begin{tabular}{|c|c|c|c|c|}
\hline \multicolumn{5}{|c|}{ c $\quad$ Floor1 Room 507} \\
\hline 507015 & 0.00359833 & $(-50701)$ & imp:n=1 & \$Metal wl \\
\hline 507025 & 0.00359833 & $(-50702)$ & imp:n=1 & \$Metal wl \\
\hline 507035 & 0.00359833 & $(-50703)$ & imp: $n=1$ & \$Metal wl \\
\hline 507045 & 0.00359833 & $(-50704)$ & imp:n=1 & \$Metal wl \\
\hline 507055 & 0.00359833 & $(-50705)$ & imp:n=1 & \$Metal wl \\
\hline 507065 & 0.00359833 & $(-50706)$ & imp:n=1 & \$Metal wl \\
\hline 507075 & 0.00359833 & $(-50707)$ & imp:n=1 & \$Metal wl \\
\hline 507085 & 0.00359833 & $(-50708)$ & imp:n=1 & \$Metal wl \\
\hline 507095 & 0.00359833 & $(-50709)$ & imp:n=1 & \$Metal wl \\
\hline 507105 & 0.00359833 & $(-50710)$ & imp:n=1 & \$Metal wl \\
\hline 507115 & 0.00359833 & $(-50711)$ & imp:n=1 & \$Metal wl \\
\hline c & & & & \\
\hline C & & & & \\
\hline c Floo & 1 Room 506 & & & \\
\hline 506015 & 0.00359833 & $(-50601)$ & imp:n=1 & \$Metal wl \\
\hline 506025 & 0.00359833 & $(-50602)$ & imp:n=1 & \$Metal wl \\
\hline 506035 & 0.00359833 & $(-50603)$ & imp:n=1 & \$Metal wl \\
\hline 506045 & 0.00359833 & $(-50604)$ & imp:n=1 & \$Metal wl \\
\hline 506055 & 0.00359833 & $(-50605)$ & imp:n=1 & \$Metal wl \\
\hline 506065 & 0.00359833 & $(-50606)$ & imp:n=1 & \$Metal wl \\
\hline 506075 & 0.00359833 & $(-50607)$ & imp:n=1 & \$Metal wl \\
\hline 506085 & 0.00359833 & $(-50608)$ & imp:n=1 & \$Metal wl \\
\hline 506095 & 0.00359833 & $(-50609)$ & imp:n=1 & \$Metal wl \\
\hline 506105 & 0.00359833 & $(-50610)$ & imp:n=1 & \$Metal wl \\
\hline 506115 & 0.00359833 & $(-50611)$ & imp:n=1 & \$Metal wl \\
\hline 506125 & 0.00359833 & $(-50612)$ & imp:n=1 & \$Metal wl \\
\hline 506135 & 0.00359833 & $(-50613)$ & imp:n=1 & \$Metal wl \\
\hline c & & & & \\
\hline c & & & & \\
\hline c Floo & 1 Room 410 & & & \\
\hline 410015 & 0.00359833 & $(-41001)$ & imp:n=1 & \$Metal wl \\
\hline 410025 & 0.00359833 & $(-41002)$ & imp:n=1 & \$Metal wl \\
\hline 410035 & 0.00359833 & $(-41003)$ & imp: $n=1$ & \$Metal wl \\
\hline 410045 & 0.00359833 & $(-41004)$ & imp:n=1 & \$Metal wl \\
\hline 410055 & 0.00359833 & $(-41005)$ & imp:n=1 & \$Metal wl \\
\hline 4100613 & -7.92 & $(-41006)$ & imp:n=1 & \$SS \\
\hline 410075 & 0.00359833 & $(-41007)$ & imp: $n=1$ & \$Metal wl \\
\hline 410085 & 0.00359833 & $(-41008)$ & imp:n=1 & \$Metal wl \\
\hline 410095 & 0.00359833 & $(-41009)$ & imp:n=1 & \$Metal wl \\
\hline 410105 & 0.00359833 & $(-41010)$ & imp:n=1 & \$Metal wl \\
\hline 410115 & 0.00359833 & $(-41011)$ & imp:n=1 & \$Metal wl \\
\hline 410125 & 0.00359833 & $(-41012)$ & imp:n=1 & \$Metal wl \\
\hline 410135 & 0.00359833 & $(-41013)$ & imp:n=1 & \$Metal wl \\
\hline c & & & & \\
\hline c & & & & \\
\hline c Floor & 1 Room 511 & & & \\
\hline 511015 & 0.00359833 & $(-51101)$ & imp:n=1 & \$Metal wl \\
\hline 511025 & 0.00359833 & $(-51102)$ & imp:n=1 & \$Metal wl \\
\hline 511035 & 0.00359833 & $(-51103)$ & imp:n=1 & \$Metal wl \\
\hline 511045 & 0.00359833 & $(-51104)$ & imp:n=1 & \$Metal wl \\
\hline 511055 & 0.00359833 & $(-51105)$ & imp:n=1 & \$Metal wl \\
\hline 511065 & 0.00359833 & $(-51106)$ & imp:n=1 & \$Metal wl \\
\hline 511075 & 0.00359833 & $(-51107)$ & imp:n=1 & \$Metal wl \\
\hline 511085 & 0.00359833 & $(-51108)$ & imp:n=1 & \$Metal wl \\
\hline 511095 & 0.00359833 & $(-51109)$ & imp:n=1 & \$Metal wl \\
\hline c & & & & \\
\hline c & & & & \\
\hline Floor & 1 Room 510 & & & \\
\hline 510015 & 0.00359833 & $(-51001)$ & imp:n=1 & \$Metal wl \\
\hline 510025 & 0.00359833 & $(-51002)$ & imp:n=1 & \$Metal wl \\
\hline 510035 & 0.00359833 & $(-51003)$ & imp:n=1 & \$Metal wl \\
\hline 510045 & 0.00359833 & $(-51004)$ & imp:n=1 & \$Metal wl \\
\hline 510055 & 0.00359833 & $(-51005)$ & imp:n=1 & \$Metal wl \\
\hline 510065 & 0.00359833 & $(-51006)$ & imp:n=1 & \$Metal wl \\
\hline 510075 & 0.00359833 & $(-51007)$ & imp:n=1 & \$Metal wl \\
\hline 510085 & 0.00359833 & $(-51008)$ & imp:n=1 & \$Metal wl \\
\hline 510095 & 0.00359833 & $(-51009)$ & imp:n=1 & \$Metal wl \\
\hline
\end{tabular}

c

C 


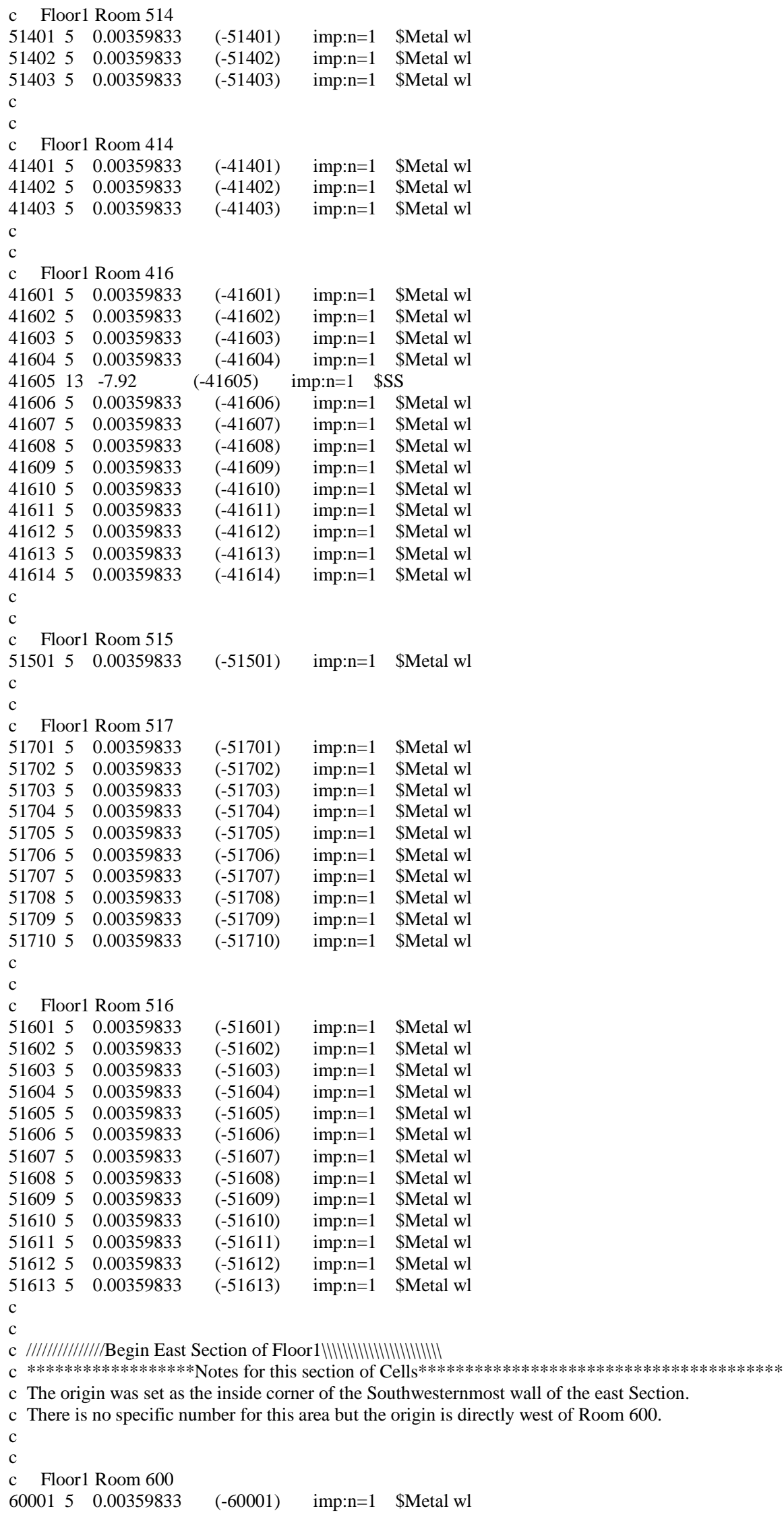




\begin{tabular}{|c|c|c|c|c|}
\hline 600023 & -2.3 & $(-60002) \quad \mathrm{i}$ & imp:n=1 & tevic rimed \\
\hline 600035 & 0.00359833 & $(-60003)$ & imp:n=1 & 1 \$Metal wl \\
\hline 600045 & 0.00359833 & $(-60004)$ & imp:n=1 & \$Metal wl \\
\hline 600053 & -2.3 & $(-60005) \quad \mathrm{i}$ & imp:n=1 $\quad \$$ & Conc Filled \\
\hline 600065 & 0.00359833 & $(-60006)$ & imp:n=1 & 1 \$Metal wl \\
\hline 600075 & 0.00359833 & $(-60007)$ & imp:n=1 & \$Metal wl \\
\hline 600085 & 0.00359833 & $(-60008)$ & imp:n=1 & \$Metal wl \\
\hline 600095 & 0.00359833 & $(-60009)$ & imp:n=1 & \$Metal wl \\
\hline 600105 & 0.00359833 & $(-60010)$ & imp:n=1 & \$Metal wl \\
\hline 600115 & 0.00359833 & $(-60011)$ & imp:n=1 & \$Metal wl \\
\hline 600125 & 0.00359833 & $(-60012)$ & imp:n=1 & \$Metal wl \\
\hline 600135 & 0.00359833 & $(-60013)$ & imp:n=1 & \$Metal wl \\
\hline 600145 & 0.00359833 & $(-60014)$ & imp:n=1 & \$Metal wl \\
\hline 600155 & 0.00359833 & $(-60015)$ & imp:n=1 & \$Metal wl \\
\hline $60017 \quad 15$ & -0.4751 & $(-60017)$ & imp:n=1 & \$Ext wl Filled \\
\hline$C$ & & & & \\
\hline & & & & \\
\hline Floor & 1 Room 602/6 & 605/606 & & \\
\hline & nese rooms we & ere all combin & ned because & ***** \\
\hline & & & & \\
\hline 602015 & 0.00359833 & $(-60201)$ & imp:n=1 & \$Metal wl \\
\hline 602025 & 0.00359833 & $(-60202)$ & imp:n=1 & \$Metal wl \\
\hline 602035 & 0.00359833 & $(-60203)$ & imp:n=1 & \$Metal wl \\
\hline 602045 & 0.00359833 & $(-60204)$ & imp:n=1 & \$Metal wl \\
\hline 602055 & 0.00359833 & $(-60205)$ & imp:n=1 & \$Metal wl \\
\hline 602065 & 0.00359833 & $(-60206)$ & imp:n=1 & \$Metal wl \\
\hline 602075 & 0.00359833 & $(-60207)$ & imp:n=1 & \$Metal wl \\
\hline 602085 & 0.00359833 & $(-60208)$ & imp:n=1 & \$Metal wl \\
\hline 602095 & 0.00359833 & $(-60209)$ & imp:n=1 & \$Metal wl \\
\hline 602105 & 0.00359833 & $(-60210)$ & imp:n=1 & \$Metal wl \\
\hline 602115 & 0.00359833 & $(-60211)$ & imp:n=1 & \$Metal wl \\
\hline 602125 & 0.00359833 & $(-60212)$ & imp:n=1 & \$Metal wl \\
\hline 602135 & 0.00359833 & $(-60213)$ & imp:n=1 & \$Metal wl \\
\hline 602145 & 0.00359833 & $(-60214)$ & imp:n=1 & \$Metal wl \\
\hline 602155 & 0.00359833 & $(-60215)$ & imp:n=1 & \$Metal wl \\
\hline 602165 & 0.00359833 & $(-60216)$ & imp:n=1 & \$Metal wl \\
\hline 602175 & 0.00359833 & $(-60217)$ & imp:n=1 & \$Metal wl \\
\hline 602185 & 0.00359833 & $(-60218)$ & imp:n=1 & \$Metal wl \\
\hline 602195 & 0.00359833 & $(-60219)$ & imp:n=1 & \$Metal wl \\
\hline 602205 & 0.00359833 & $(-60220)$ & imp:n=1 & \$Metal wl \\
\hline 602215 & 0.00359833 & $(-60221)$ & imp:n=1 & \$Metal wl \\
\hline 602225 & 0.00359833 & $(-60222)$ & imp:n=1 & \$Metal wl \\
\hline 602235 & 0.00359833 & $(-60223)$ & imp:n=1 & \$Metal wl \\
\hline 602245 & 0.00359833 & $(-60224)$ & imp:n=1 & \$Metal wl \\
\hline 602255 & 0.00359833 & $(-60225)$ & imp:n=1 & \$Metal wl \\
\hline 602265 & 0.00359833 & $(-60226)$ & imp:n=1 & \$Metal wl \\
\hline 602275 & 0.00359833 & $(-60227)$ & imp:n=1 & \$Metal wl \\
\hline 602285 & 0.00359833 & $(-60228)$ & imp:n=1 & \$Metal wl \\
\hline 6023215 & -0.4751 & $(-60232)$ & imp:n=1 & \$Ext wl Filled \\
\hline c & & & & \\
\hline c & & & & \\
\hline Floo & 1 Room $610 \mathrm{~T}$ & Truck Lock & & \\
\hline & ${ }^{* *}$ Note: a $.5 \mathrm{~cm}$ & m thick materi & rial t & \\
\hline 6100115 & -0.4751 & $(-61001)$ & imp:n=1 & \$Ext wl Filled \\
\hline 610025 & 0.00359833 & $(-61002)$ & imp:n=1 & 1 \$Metal wl \\
\hline 610035 & 0.00359833 & $\quad(-61003)$ & imp:n=1 & \$Metal wl \\
\hline 6100413 & -7.92 & $(-61004)$ & imp:n=1 & \$SS \\
\hline 6100513 & -7.92 & $(-61005)$ & imp:n=1 & \$SS \\
\hline 6100613 & -7.92 & $(-61006)$ & imp:n=1 & \$SS \\
\hline 610075 & 0.00359833 & $\quad(-61007)$ & imp:n=1 & \$Metal wl \\
\hline 610085 & 0.00359833 & $(-61008)$ & imp:n=1 & \$Metal wl \\
\hline 610095 & 0.00359833 & $(-61009)$ & imp:n=1 & \$Metal wl \\
\hline 610105 & 0.00359833 & $(-61010)$ & imp:n=1 & \$Metal wl \\
\hline 610115 & 0.00359833 & $(-61011)$ & imp:n=1 & \$Metal wl \\
\hline 610125 & 0.00359833 & $(-61012)$ & imp:n=1 & \$Metal wl \\
\hline 610135 & 0.00359833 & $(-61013)$ & imp:n=1 & \$Metal wl \\
\hline 610145 & 0.00359833 & $\quad(-61014)$ & imp:n=1 & 1 \$Metal wl \\
\hline 6101513 & -7.92 & $(-61015)$ & imp:n=1 & \$SS \\
\hline 610165 & 0.00359833 & $\quad(-61016)$ & imp:n=1 & 1 \$Metal wl \\
\hline 610175 & 0.00359833 & $(-61017)$ & imp:n=1 & \$Metal wl \\
\hline
\end{tabular}




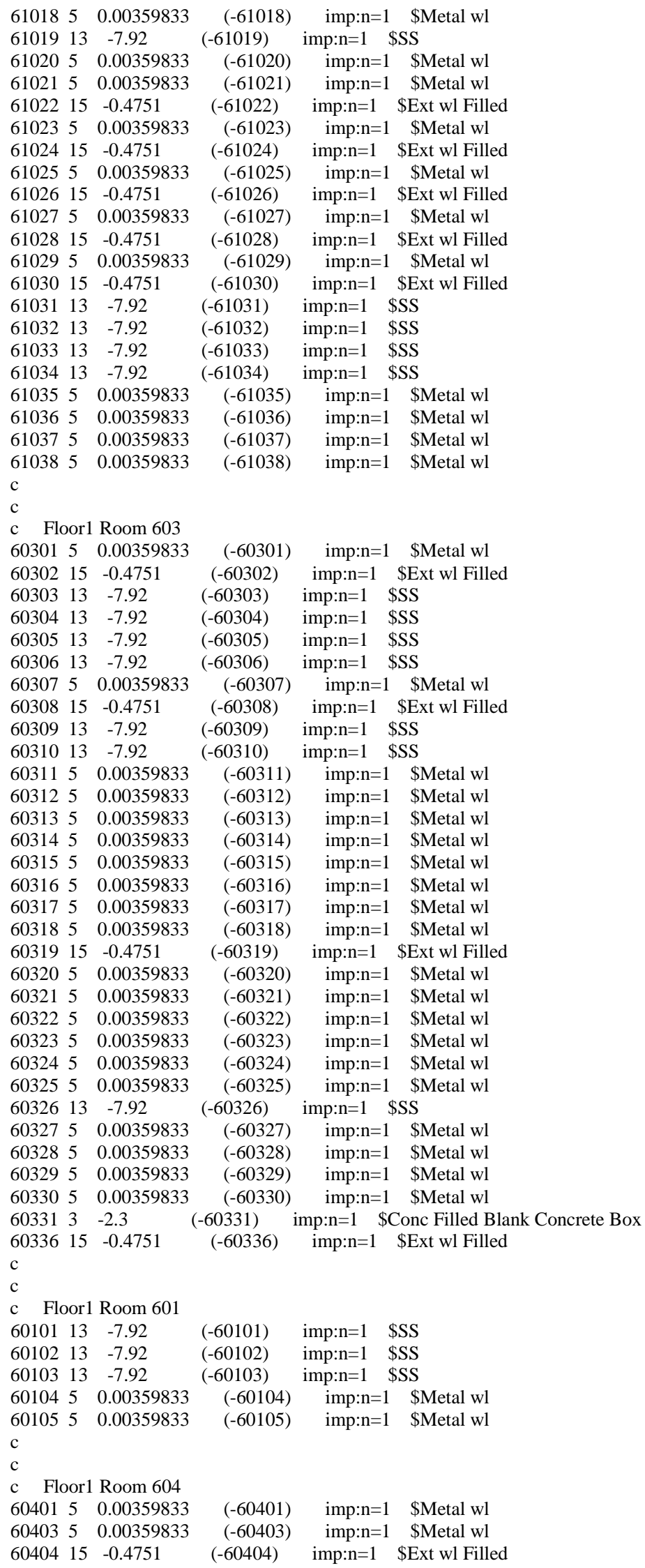




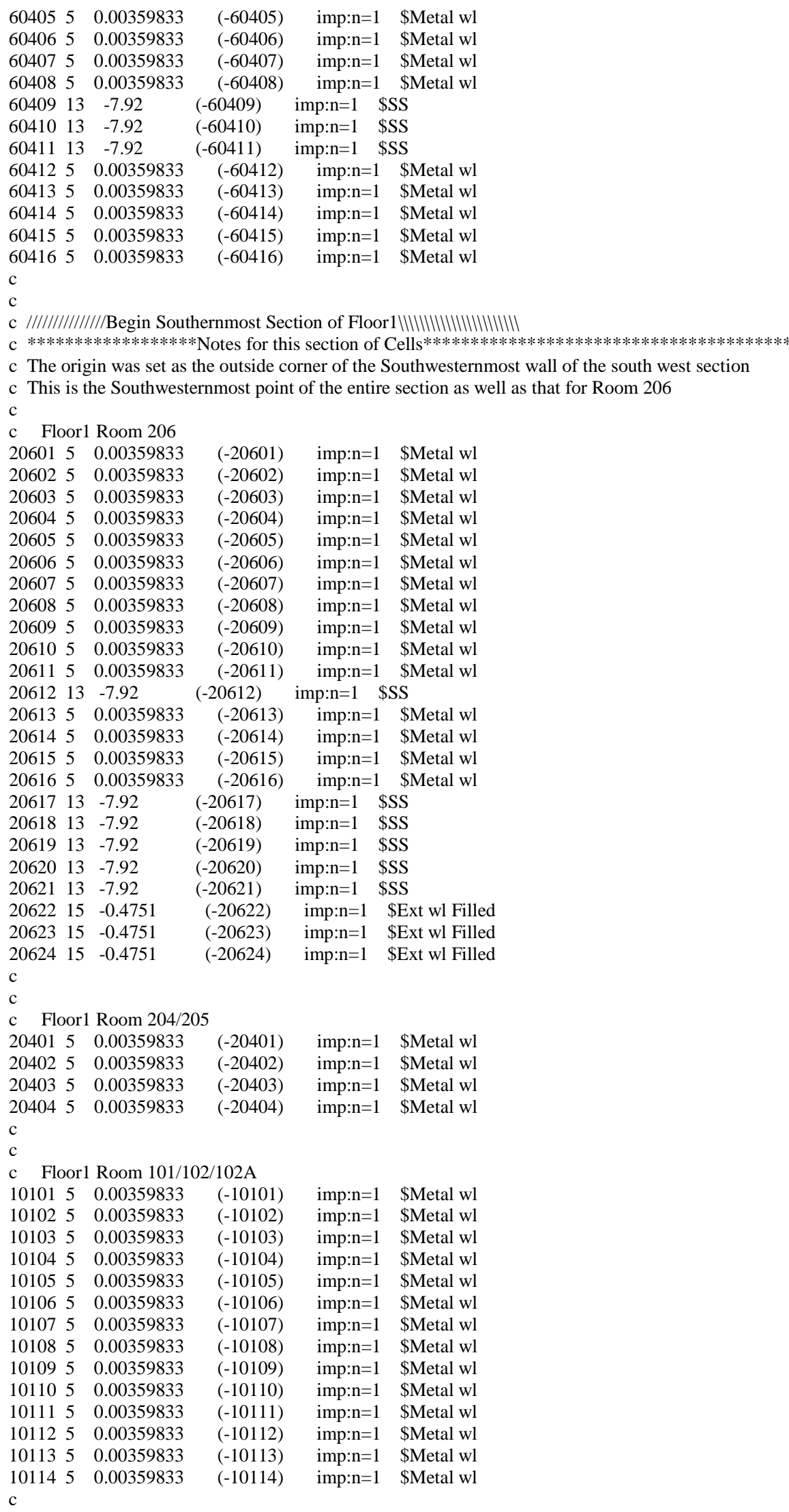




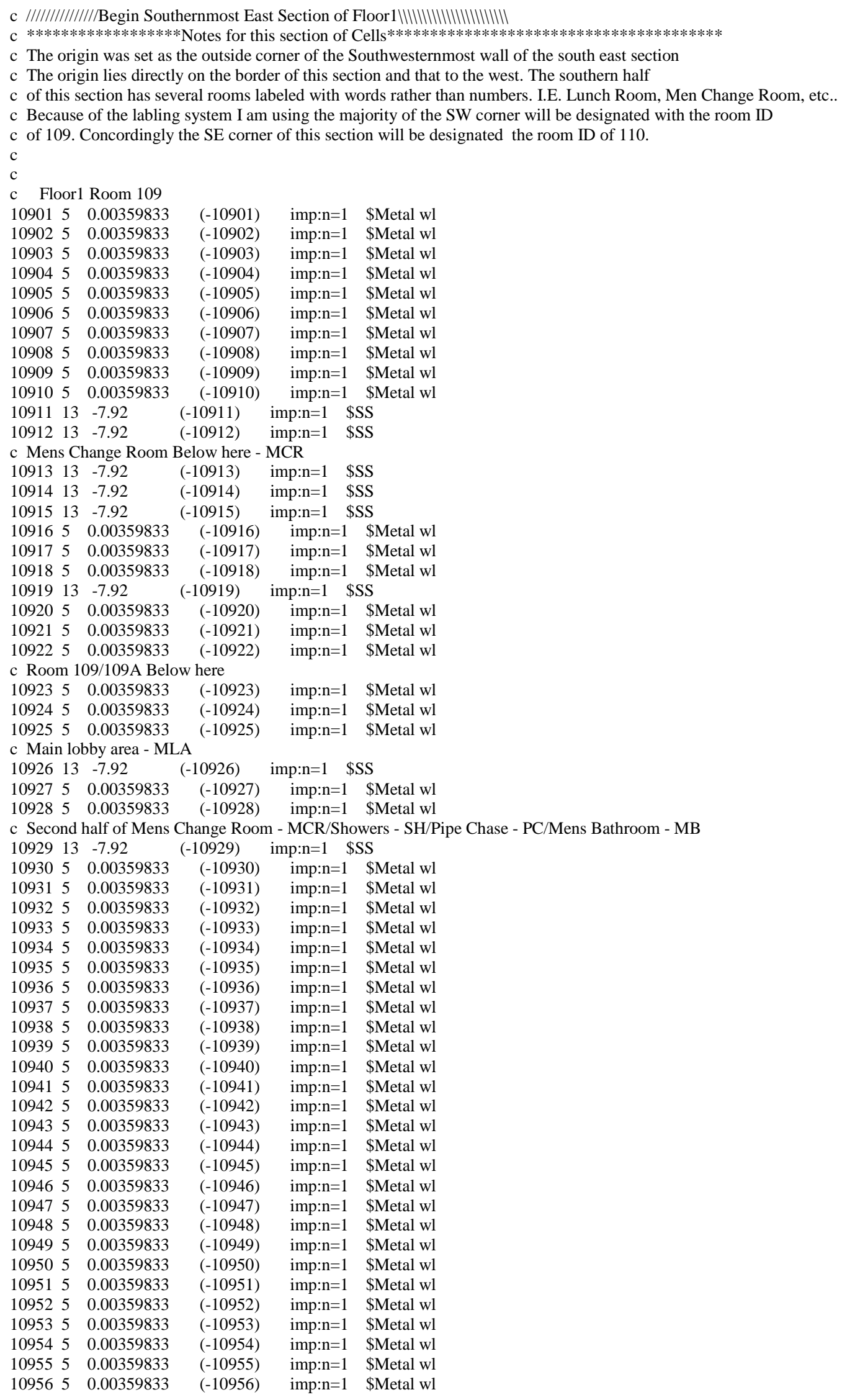




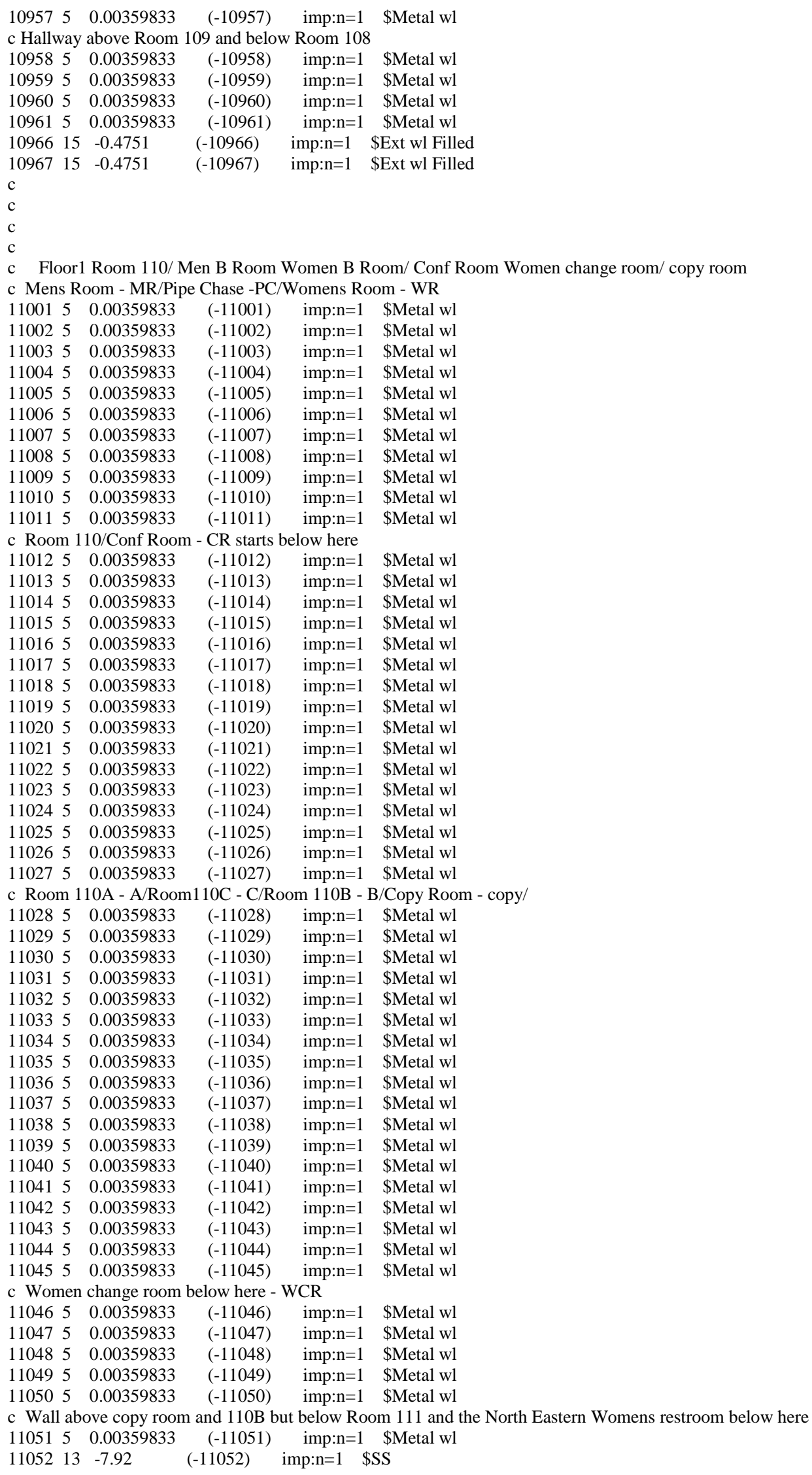




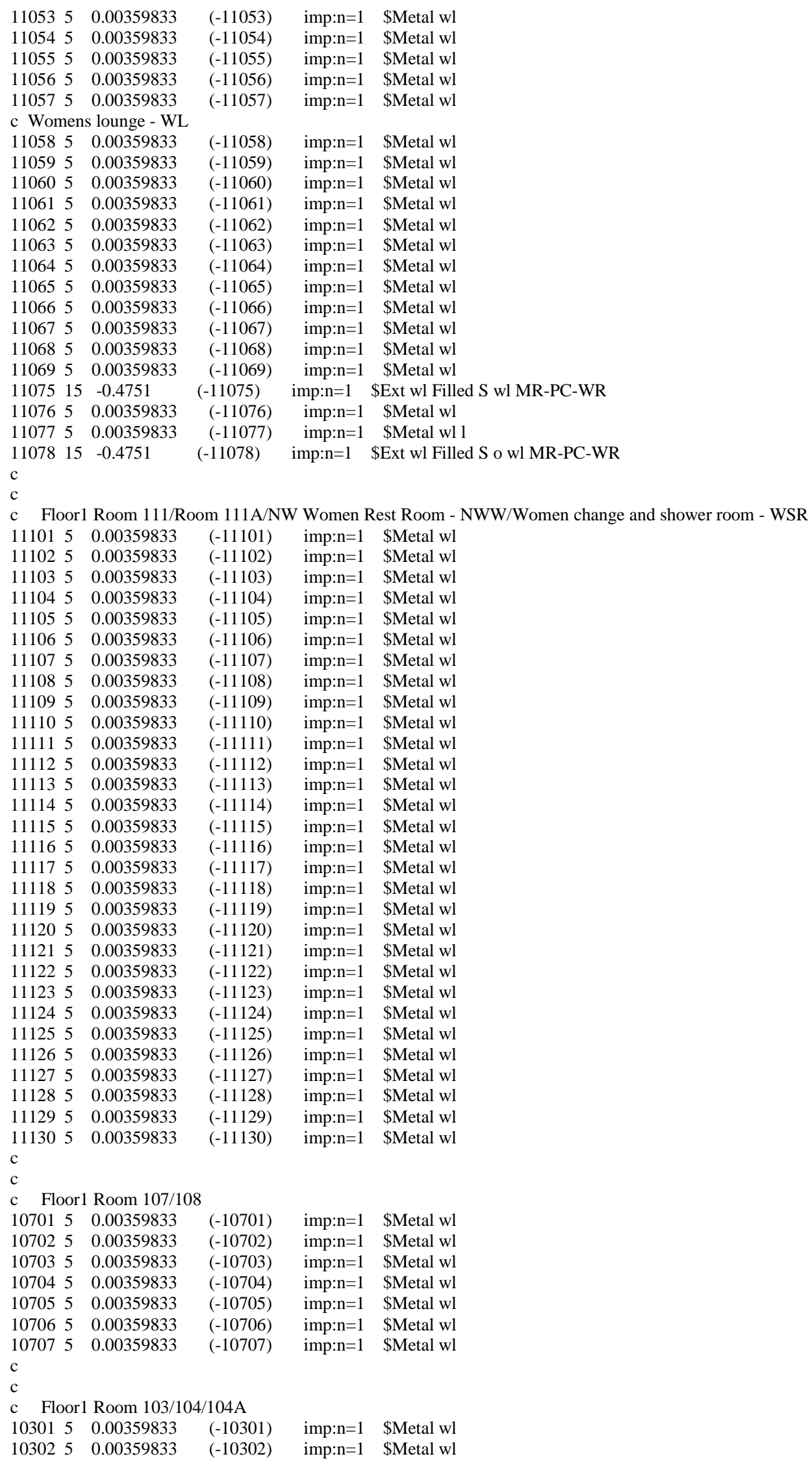




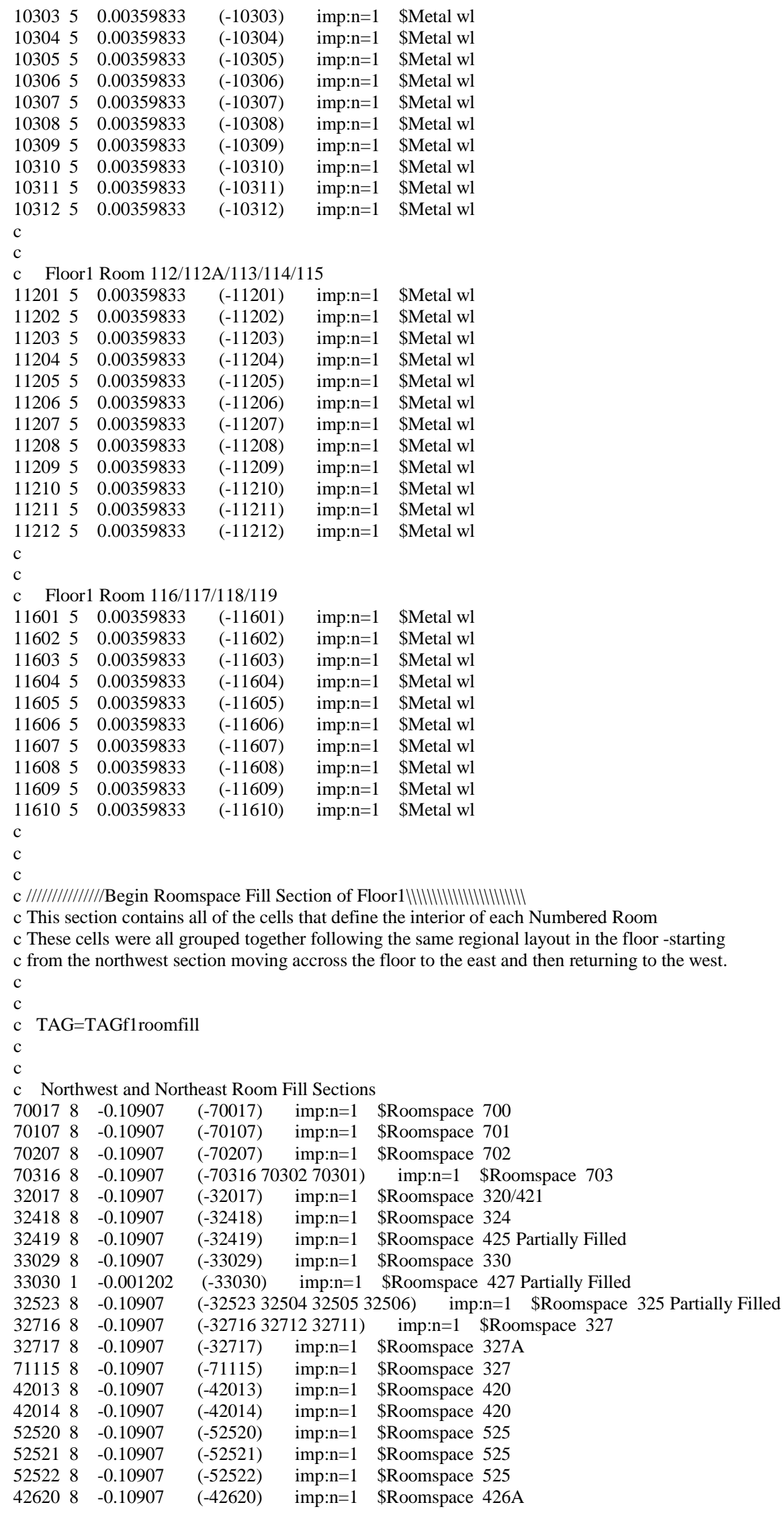




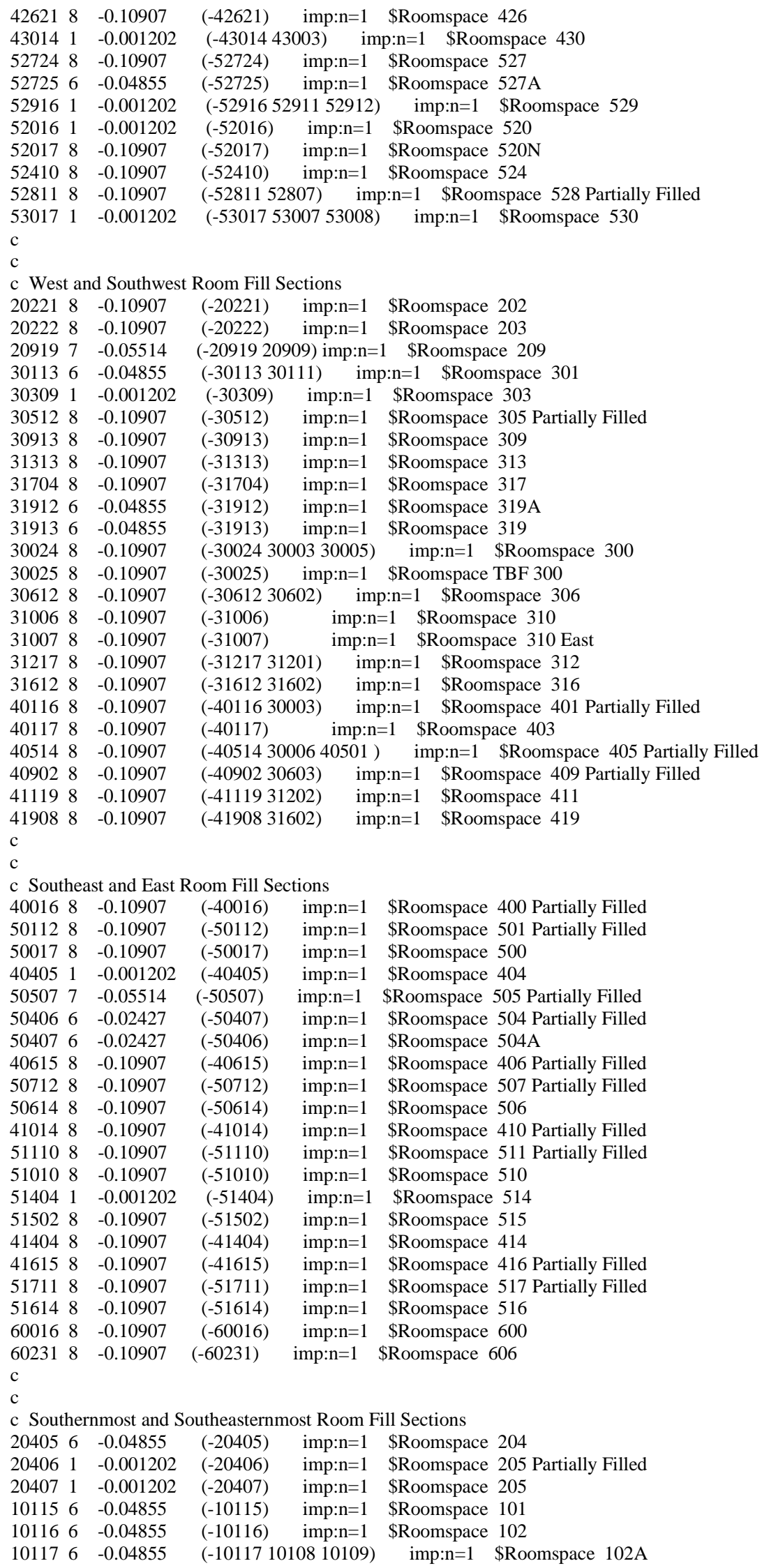




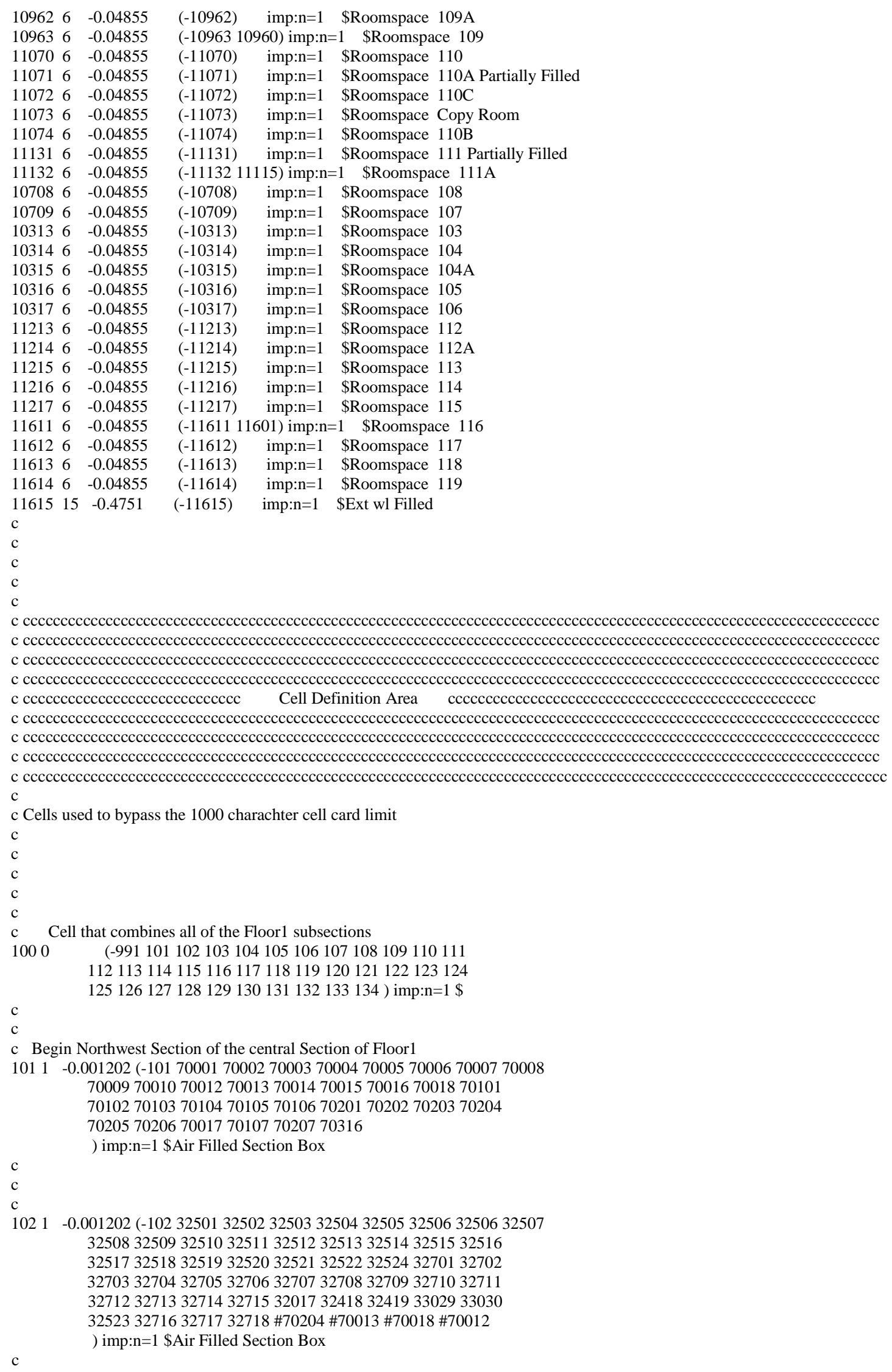




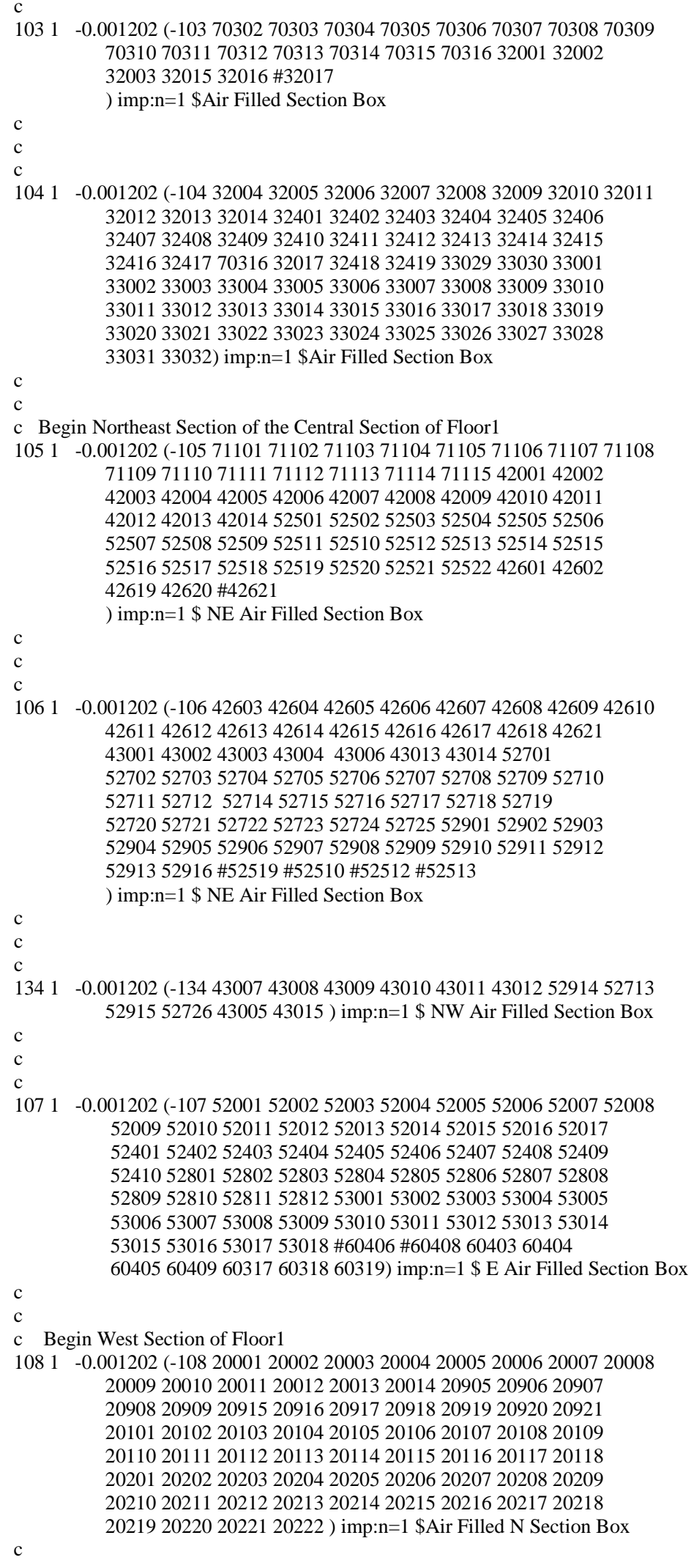




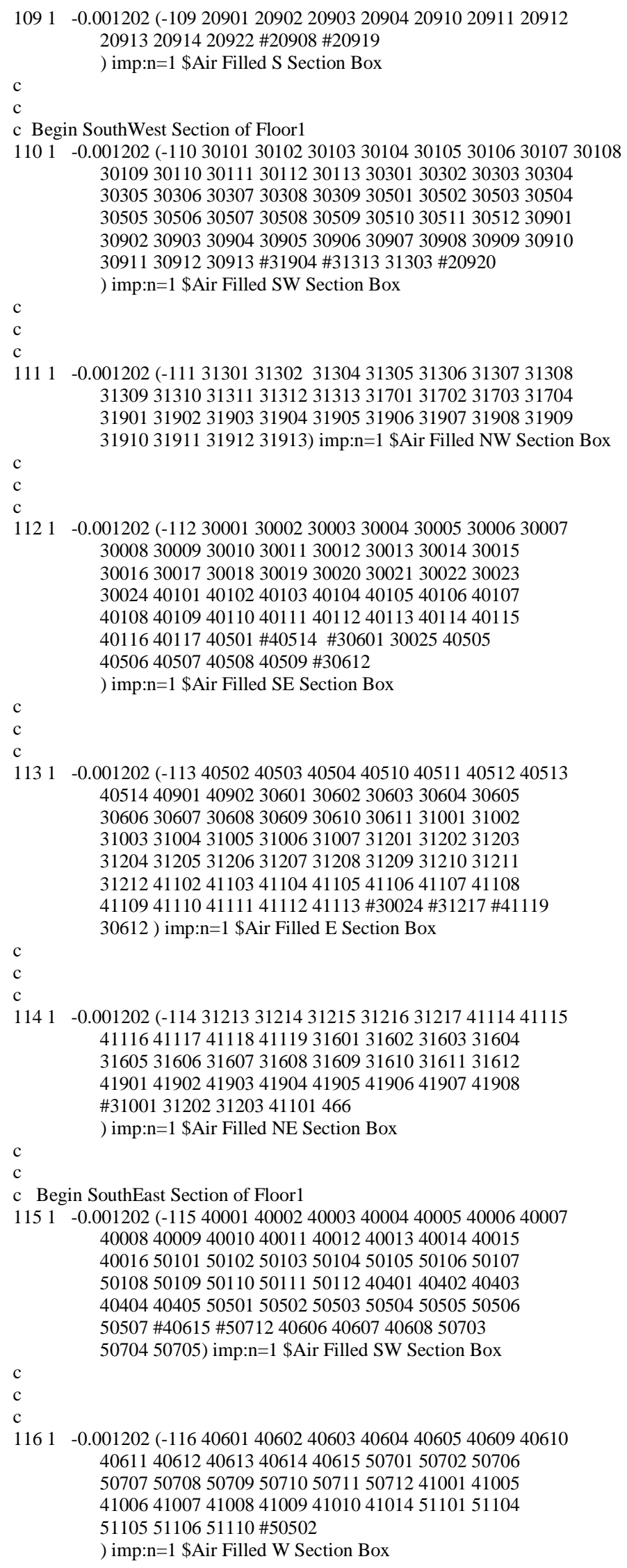




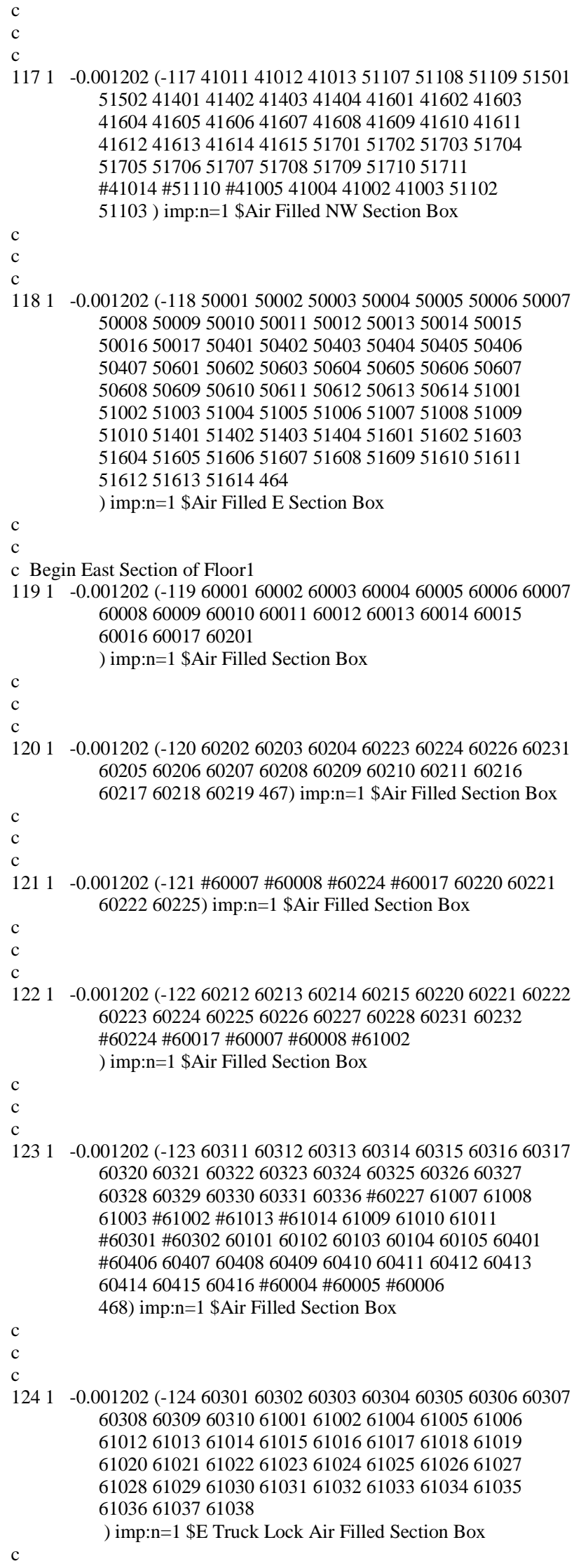




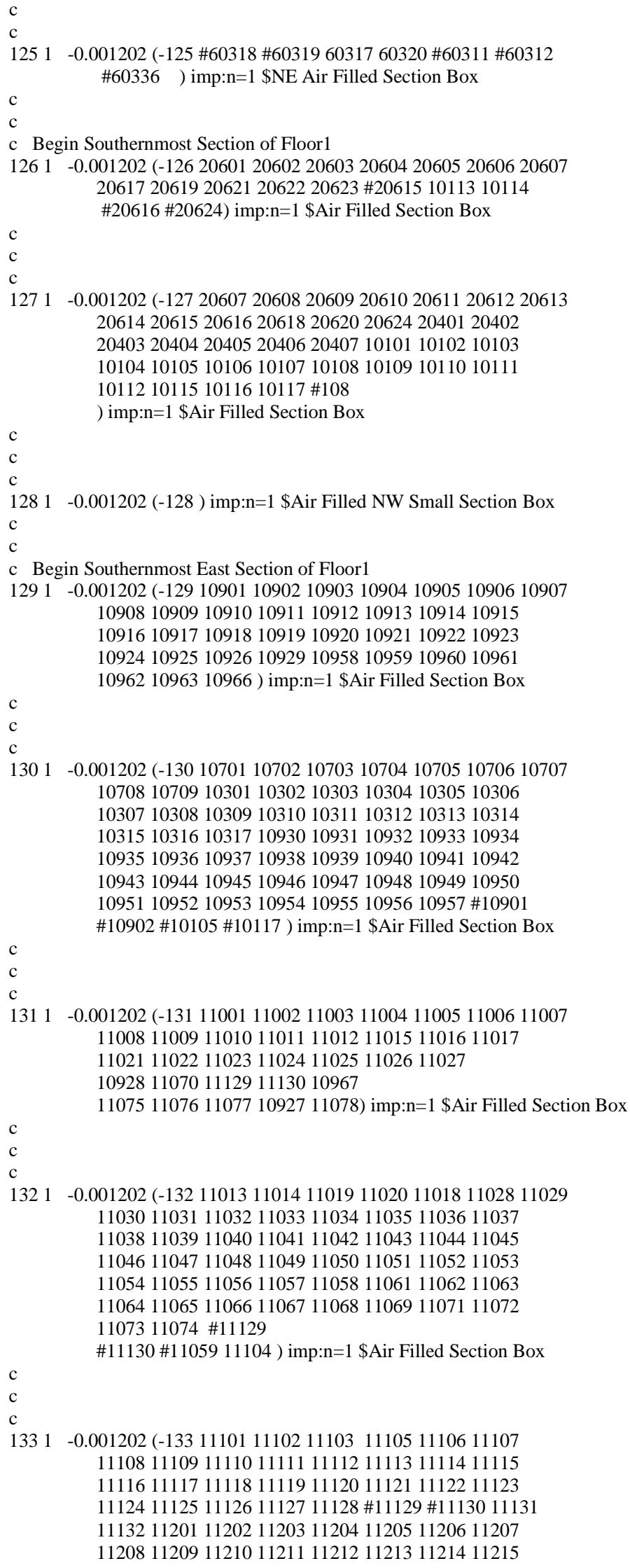


1121611217116011160211603116041160511606

1160711608116091161011611116121161311614

116151105911060 \#11061 \#11062 465

) imp:n=1 \$Air Filled Section Box 


\begin{tabular}{|c|c|c|c|c|c|}
\hline 91803 & 5 & 0.00359833 & $(-91803)$ & imp:n=1 & \$Metal wl \\
\hline 91804 & 5 & 0.00359833 & $(-91804)$ & imp:n=1 & \$Metal wl \\
\hline 91805 & 5 & 0.00359833 & $(-91805)$ & imp:n=1 & \$Metal wl \\
\hline 91806 & 5 & 0.00359833 & $(-91806)$ & imp:n=1 & \$Metal wl \\
\hline 91807 & 5 & 0.00359833 & $(-91807)$ & imp:n=1 & \$Metal wl \\
\hline 91808 & 5 & 0.00359833 & $(-91808)$ & imp:n=1 & \$Metal wl \\
\hline 91809 & 5 & 0.00359833 & $(-91809)$ & imp:n=1 & \$Metal wl \\
\hline 91810 & 5 & 0.00359833 & $(-91810)$ & imp:n=1 & \$Metal wl \\
\hline 91811 & 5 & 0.00359833 & $(-91811)$ & imp:n=1 & \$Metal wl \\
\hline 91812 & 5 & 0.00359833 & $(-91812)$ & imp:n=1 & \$Metal wl \\
\hline 91813 & 5 & 0.00359833 & $(-91813)$ & imp:n=1 & \$Metal wl \\
\hline 91814 & 5 & 0.00359833 & $(-91814)$ & imp:n=1 & \$Metal wl \\
\hline 91815 & 5 & 0.00359833 & $(-91815)$ & imp:n=1 & \$Metal wl \\
\hline 91816 & 5 & 0.00359833 & $(-91816)$ & imp:n=1 & \$Metal wl \\
\hline 91817 & 5 & 0.00359833 & $(-91817)$ & imp:n=1 & \$Metal wl \\
\hline 91818 & 5 & 0.00359833 & $(-91818)$ & imp:n=1 & \$Metal wl \\
\hline 91819 & 15 & -0.4751 & $(-91819)$ & imp:n=1 & \$Ext wl \\
\hline 91820 & 5 & 0.00359833 & $(-91820)$ & imp:n=1 & \$Metal wl \\
\hline C & & & & & \\
\hline C & & & & & \\
\hline c Flo & or2 & Room 927/93 & 3/935/926/93 & 7/925/939 s & ection contai \\
\hline 92701 & 5 & 0.00359833 & $(-92701)$ & imp:n=1 & \$Metal wl \\
\hline 92702 & 5 & 0.00359833 & $(-92702)$ & imp:n=1 & \$Metal wl \\
\hline 92703 & 5 & 0.00359833 & $(-92703)$ & imp:n=1 & \$Metal wl \\
\hline 92704 & 5 & 0.00359833 & $(-92704)$ & imp:n=1 & \$Metal wl \\
\hline 92705 & 5 & 0.00359833 & $(-92705)$ & imp:n=1 & \$Metal wl \\
\hline 92706 & 5 & 0.00359833 & $(-92706)$ & imp:n=1 & \$Metal wl \\
\hline 92707 & 5 & 0.00359833 & $(-92707)$ & imp:n=1 & \$Metal wl \\
\hline 92708 & 5 & 0.00359833 & $(-92708)$ & imp:n=1 & \$Metal wl \\
\hline 92709 & 5 & 0.00359833 & $(-92709)$ & imp:n=1 & \$Metal wl \\
\hline 92710 & 5 & 0.00359833 & $(-92710)$ & imp:n=1 & \$Metal wl \\
\hline 92711 & 5 & 0.00359833 & $(-92711)$ & imp:n=1 & \$Metal wl \\
\hline 92712 & 5 & 0.00359833 & $(-92712)$ & imp:n=1 & \$Metal wl \\
\hline 92713 & 5 & 0.00359833 & $(-92713)$ & imp:n=1 & \$Metal wl \\
\hline 92714 & 5 & 0.00359833 & $(-92714)$ & imp:n=1 & \$Metal wl \\
\hline 92715 & 5 & 0.00359833 & $(-92715)$ & imp:n=1 & \$Metal wl \\
\hline 92716 & 5 & 0.00359833 & $(-92716)$ & imp:n=1 & \$Metal wl \\
\hline 92717 & 5 & 0.00359833 & $(-92717)$ & imp:n=1 & \$Metal wl \\
\hline 92718 & 5 & 0.00359833 & $(-92718)$ & imp:n=1 & \$Metal wl \\
\hline 92719 & 5 & 0.00359833 & $(-92719)$ & imp:n=1 & \$Metal wl \\
\hline 92720 & 5 & 0.00359833 & $(-92720)$ & imp:n=1 & \$Metal wl \\
\hline 92721 & 5 & 0.00359833 & $(-92721)$ & imp:n=1 & \$Metal wl \\
\hline 92722 & 5 & 0.00359833 & $(-92722)$ & imp:n=1 & \$Metal wl \\
\hline 92723 & 5 & 0.00359833 & $(-92723)$ & imp:n=1 & \$Metal wl \\
\hline 92724 & 5 & 0.00359833 & $(-92724)$ & imp:n=1 & \$Metal wl \\
\hline 92725 & 5 & 0.00359833 & $(-92725)$ & imp:n=1 & \$Metal wl \\
\hline 92726 & 5 & 0.00359833 & $(-92726)$ & imp:n=1 & \$Metal wl \\
\hline 92727 & 5 & 0.00359833 & $(-92727)$ & imp:n=1 & \$Metal wl \\
\hline c & & & & & \\
\hline C & & & & & \\
\hline c Flo & or2 & Room 920/92 & 7/924/968/96 & 5/967/964 & \\
\hline 92001 & 5 & 0.00359833 & $(-92001)$ & imp:n=1 & \$Metal wl \\
\hline 92002 & 5 & 0.00359833 & $(-92002)$ & imp:n=1 & \$Metal wl \\
\hline 92003 & 5 & 0.00359833 & $(-92003)$ & imp:n=1 & \$Metal wl \\
\hline 92004 & 5 & 0.00359833 & $(-92004)$ & imp:n=1 & \$Metal wl \\
\hline 92005 & 5 & 0.00359833 & $(-92005)$ & imp:n=1 & \$Metal wl \\
\hline 92006 & 5 & 0.00359833 & $(-92006)$ & imp:n=1 & \$Metal wl \\
\hline 92007 & 5 & 0.00359833 & $(-92007)$ & imp:n=1 & \$Metal wl \\
\hline 92008 & 5 & 0.00359833 & $(-92008)$ & imp:n=1 & \$Metal wl \\
\hline 92009 & 5 & 0.00359833 & $(-92009)$ & imp:n=1 & \$Metal wl \\
\hline 92010 & 5 & 0.00359833 & $(-92010)$ & imp:n=1 & \$Metal wl \\
\hline 92011 & 5 & 0.00359833 & $(-92011)$ & imp:n=1 & \$Metal wl \\
\hline 92012 & 5 & 0.00359833 & $(-92012)$ & imp:n=1 & \$Metal wl \\
\hline 92013 & 5 & 0.00359833 & $(-92013)$ & imp:n=1 & \$Metal wl \\
\hline 92014 & 5 & 0.00359833 & $(-92014)$ & imp:n=1 & \$Metal wl \\
\hline 92015 & 5 & 0.00359833 & $(-92015)$ & imp:n=1 & \$Metal wl \\
\hline 92016 & 5 & 0.00359833 & $(-92016)$ & imp:n=1 & \$Metal wl \\
\hline 92017 & 5 & 0.00359833 & $(-92017)$ & imp:n=1 & \$Metal wl \\
\hline 92018 & 5 & 0.00359833 & $(-92018)$ & imp:n=1 & \$Metal wl \\
\hline 92019 & 5 & 0.00359833 & $(-92019)$ & imp:n=1 & \$Metal wl \\
\hline
\end{tabular}




\begin{tabular}{|c|c|c|c|c|c|}
\hline 92020 & 5 & 0.00359833 & $(-92020)$ & imp:n=1 & \$Metal wl \\
\hline 92021 & 5 & 0.00359833 & $(-92021)$ & imp:n=1 & \$Metal wl \\
\hline 92022 & 5 & 0.00359833 & $(-92022)$ & imp:n=1 & \$Metal wl \\
\hline 92023 & 15 & -0.4751 & $(-92023)$ & imp:n=1 & \$Exterior wl \\
\hline 92024 & 5 & 0.00359833 & $(-92024)$ & imp:n=1 & \$Metal wl \\
\hline 92025 & 5 & 0.00359833 & $(-92025)$ & imp:n=1 & \$Metal wl \\
\hline 92026 & 5 & 0.00359833 & $(-92026)$ & imp:n=1 & \$Metal wl \\
\hline C & & & & & \\
\hline C & & & & & \\
\hline $\mathrm{Fl}$ & oor2 & Room 930/93 & $/ 934 / 936 / 9$ & 8/941/943/9 & $945 / 947 / 949$ \\
\hline 93001 & 5 & 0.00359833 & $(-93001)$ & imp:n=1 & \$Metal wl \\
\hline 93002 & 5 & 0.00359833 & $(-93002)$ & imp:n=1 & \$Metal wl \\
\hline 93003 & 5 & 0.00359833 & $(-93003)$ & imp:n=1 & \$Metal wl \\
\hline 93004 & 5 & 0.00359833 & $(-93004)$ & imp:n=1 & \$Metal wl \\
\hline 93005 & 5 & 0.00359833 & $(-93005)$ & imp:n=1 & \$Metal wl \\
\hline 93006 & 5 & 0.00359833 & $(-93006)$ & imp:n=1 & \$Metal wl \\
\hline 93007 & 5 & 0.00359833 & $(-93007)$ & imp:n=1 & \$Metal wl \\
\hline 93008 & 5 & 0.00359833 & $(-93008)$ & imp:n=1 & \$Metal wl \\
\hline 93009 & 5 & 0.00359833 & $(-93009)$ & imp:n=1 & \$Metal wl \\
\hline 93010 & 5 & 0.00359833 & $(-93010)$ & imp:n=1 & \$Metal wl \\
\hline 93011 & 5 & 0.00359833 & $(-93011)$ & imp:n=1 & \$Metal wl \\
\hline 93012 & 5 & 0.00359833 & $(-93012)$ & imp:n=1 & \$Metal wl \\
\hline 93013 & 5 & 0.00359833 & $(-93013)$ & imp:n=1 & \$Metal wl \\
\hline 93014 & 5 & 0.00359833 & $(-93014)$ & imp:n=1 & \$Metal wl \\
\hline 93015 & 5 & 0.00359833 & $(-93015)$ & imp:n=1 & \$Metal wl \\
\hline 93016 & 5 & 0.00359833 & $(-93016)$ & imp:n=1 & \$Metal wl \\
\hline 93017 & 5 & 0.00359833 & $(-93017)$ & imp:n=1 & \$Metal wl \\
\hline 93018 & 5 & 0.00359833 & $(-93018)$ & imp:n=1 & \$Metal wl \\
\hline 93019 & 5 & 0.00359833 & $(-93019)$ & imp:n=1 & \$Metal wl \\
\hline 93020 & 5 & 0.00359833 & $(-93020)$ & imp:n=1 & \$Metal wl \\
\hline 93021 & 5 & 0.00359833 & $(-93021)$ & imp:n=1 & \$Metal wl \\
\hline 93022 & 5 & 0.00359833 & $(-93022)$ & imp:n=1 & \$Metal wl \\
\hline 93023 & 5 & 0.00359833 & $(-93023)$ & imp: $n=1$ & \$Metal wl \\
\hline 93024 & 5 & 0.00359833 & $(-93024)$ & imp:n=1 & \$Metal wl \\
\hline 93025 & 5 & 0.00359833 & $(-93025)$ & imp:n=1 & \$Metal wl \\
\hline 93026 & 5 & 0.00359833 & $(-93026)$ & imp:n=1 & \$Metal wl \\
\hline 93027 & 5 & 0.00359833 & $(-93027)$ & imp:n=1 & \$Metal wl \\
\hline C & & & & & \\
\hline C & & & & & \\
\hline Fl & oor2 & Room 940/9 & 104846/0 & 3/954/955/9 & 956/957/958 \\
\hline 94001 & 5 & 0.00359833 & $(-94001)$ & imp:n=1 & \$Metal wl \\
\hline 94002 & 5 & 0.00359833 & $(-94002)$ & imp:n=1 & \$Metal wl \\
\hline 94003 & 5 & 0.00359833 & $(-94003)$ & imp:n=1 & \$Metal wl \\
\hline 94004 & 5 & 0.00359833 & $(-94004)$ & imp:n=1 & \$Metal wl \\
\hline 94005 & 5 & 0.00359833 & $(-94005)$ & imp:n=1 & \$Metal wl \\
\hline 94006 & 5 & 0.00359833 & $(-94006)$ & imp:n=1 & \$Metal wl \\
\hline 94007 & 5 & 0.00359833 & $(-94007)$ & imp:n=1 & \$Metal wl \\
\hline 94008 & 5 & 0.00359833 & $(-94008)$ & imp:n=1 & \$Metal wl \\
\hline 94009 & 5 & 0.00359833 & $(-94009)$ & imp:n=1 & \$Metal wl \\
\hline 94010 & 5 & 0.00359833 & $(-94010)$ & imp:n=1 & \$Metal wl \\
\hline 94011 & 5 & 0.00359833 & $(-94011)$ & imp:n=1 & \$Metal wl \\
\hline 94012 & 5 & 0.00359833 & $(-94012)$ & imp:n=1 & \$Metal wl \\
\hline 94013 & 5 & 0.00359833 & $(-94013)$ & imp:n=1 & \$Metal wl \\
\hline 94014 & 5 & 0.00359833 & $(-94014)$ & imp:n=1 & \$Metal wl \\
\hline 94015 & 5 & 0.00359833 & $(-94015)$ & imp:n=1 & \$Metal wl \\
\hline 94016 & 5 & 0.00359833 & $(-94016)$ & imp:n=1 & \$Metal wl \\
\hline 94017 & 5 & 0.00359833 & $(-94017)$ & imp:n=1 & \$Metal wl \\
\hline 94018 & 5 & 0.00359833 & $(-94018)$ & imp:n=1 & \$Metal wl \\
\hline 94019 & 5 & 0.00359833 & $(-94019)$ & imp:n=1 & \$Metal wl \\
\hline 94020 & 5 & 0.00359833 & $(-94020)$ & imp:n=1 & \$Metal wl \\
\hline 94021 & 5 & 0.00359833 & $(-94021)$ & imp:n=1 & \$Metal wl \\
\hline 94022 & 5 & 0.00359833 & $(-94022)$ & imp:n=1 & \$Metal wl \\
\hline 94023 & 5 & 0.00359833 & $(-94023)$ & imp:n=1 & \$Metal wl \\
\hline 94024 & 5 & 0.00359833 & $(-94024)$ & imp:n=1 & \$Metal wl \\
\hline 94025 & 5 & 0.00359833 & $(-94025)$ & imp:n=1 & \$Metal wl \\
\hline 94026 & 5 & 0.00359833 & $(-94026)$ & imp:n=1 & \$Metal wl \\
\hline 94027 & 5 & 0.00359833 & $(-94027)$ & imp:n=1 & \$Metal wl \\
\hline 94028 & 5 & 0.00359833 & $(-94028)$ & imp:n=1 & \$Metal wl \\
\hline 94029 & 5 & 0.00359833 & $(-94029)$ & imp:n=1 & \$Metal wl \\
\hline
\end{tabular}




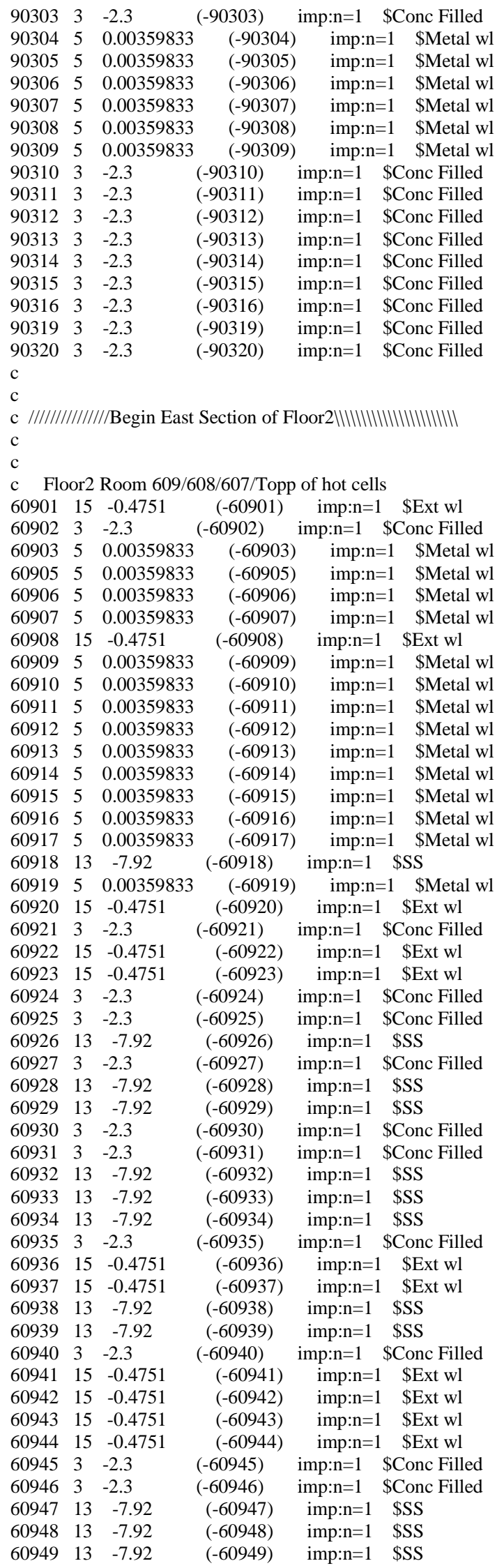




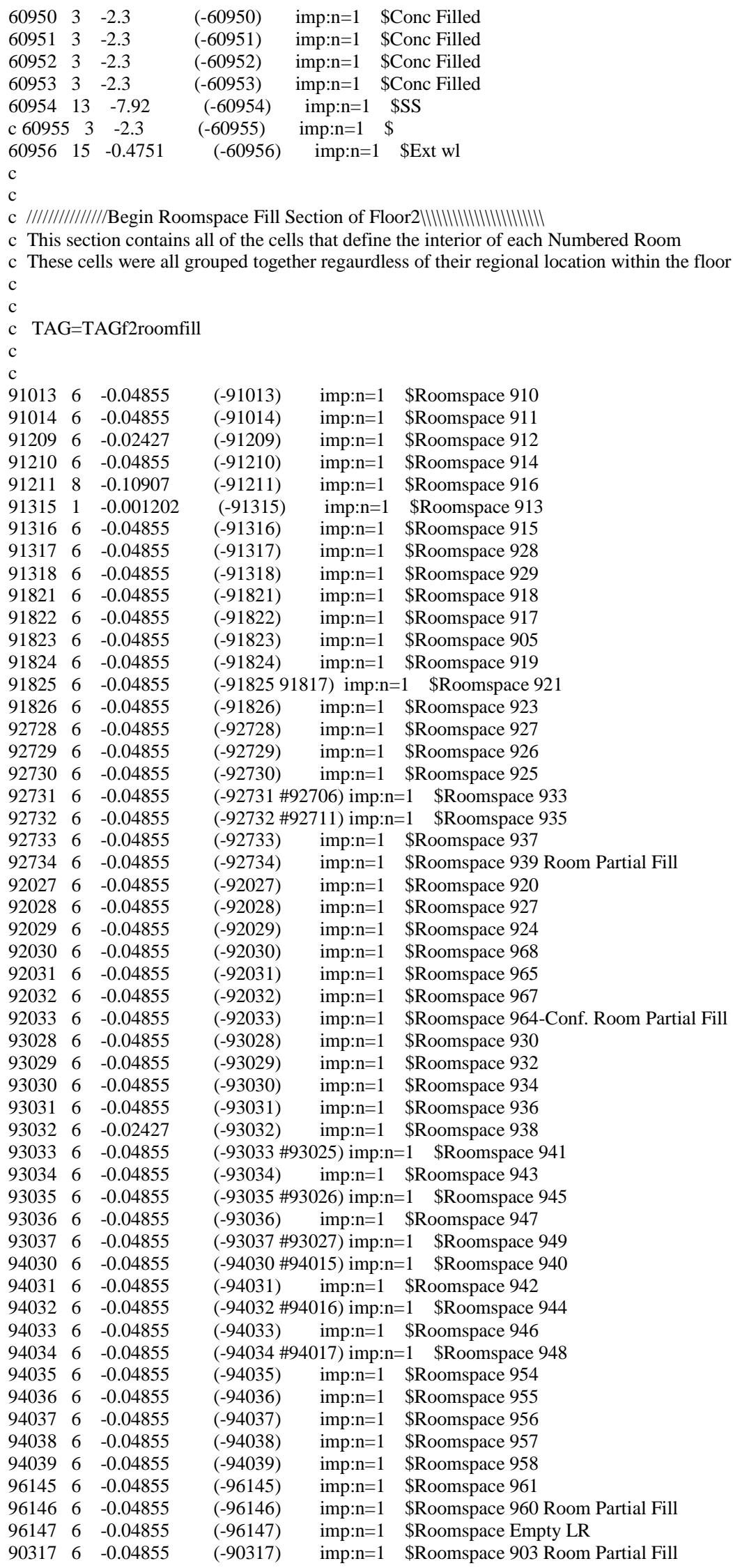




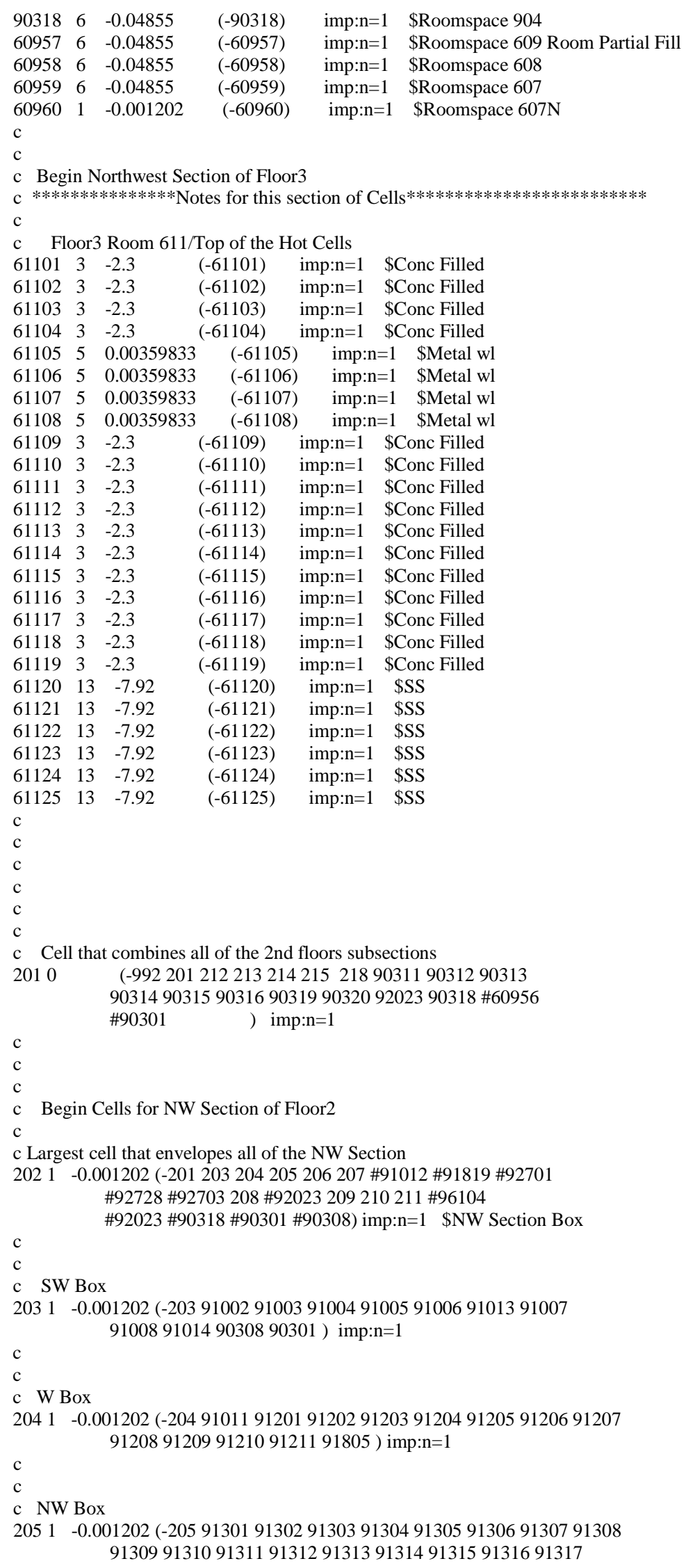




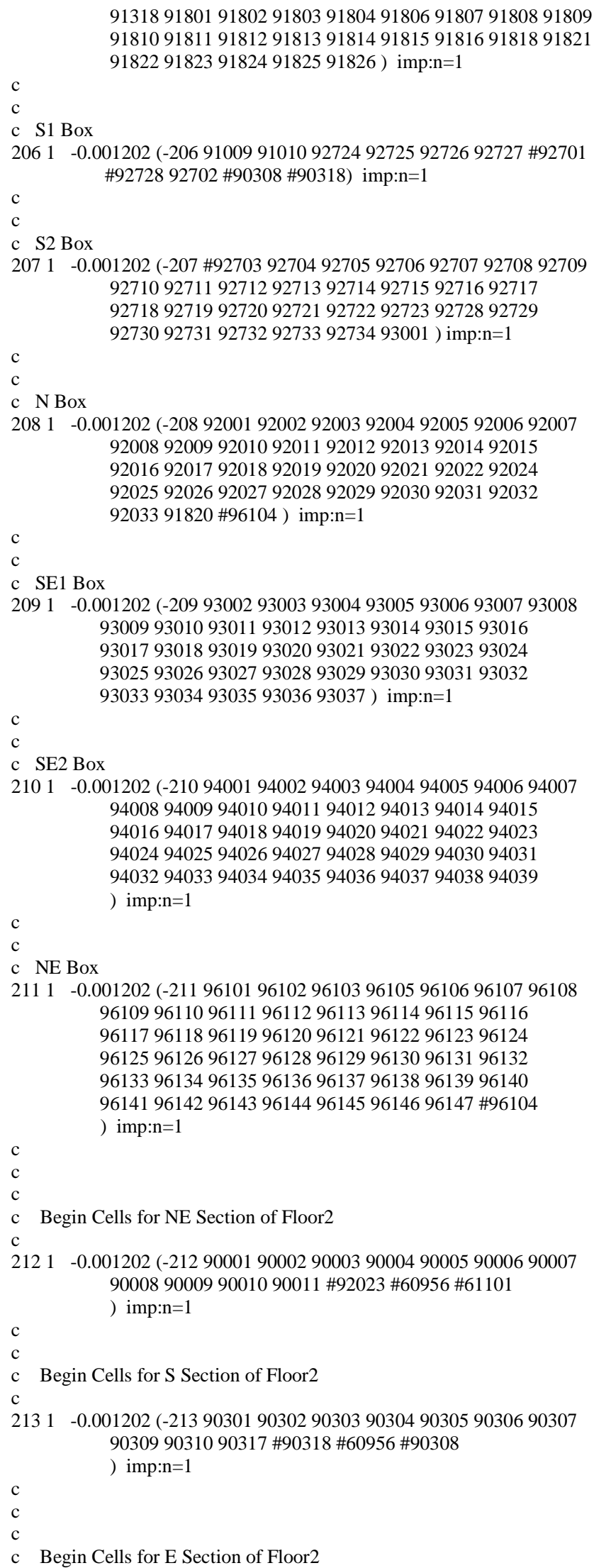




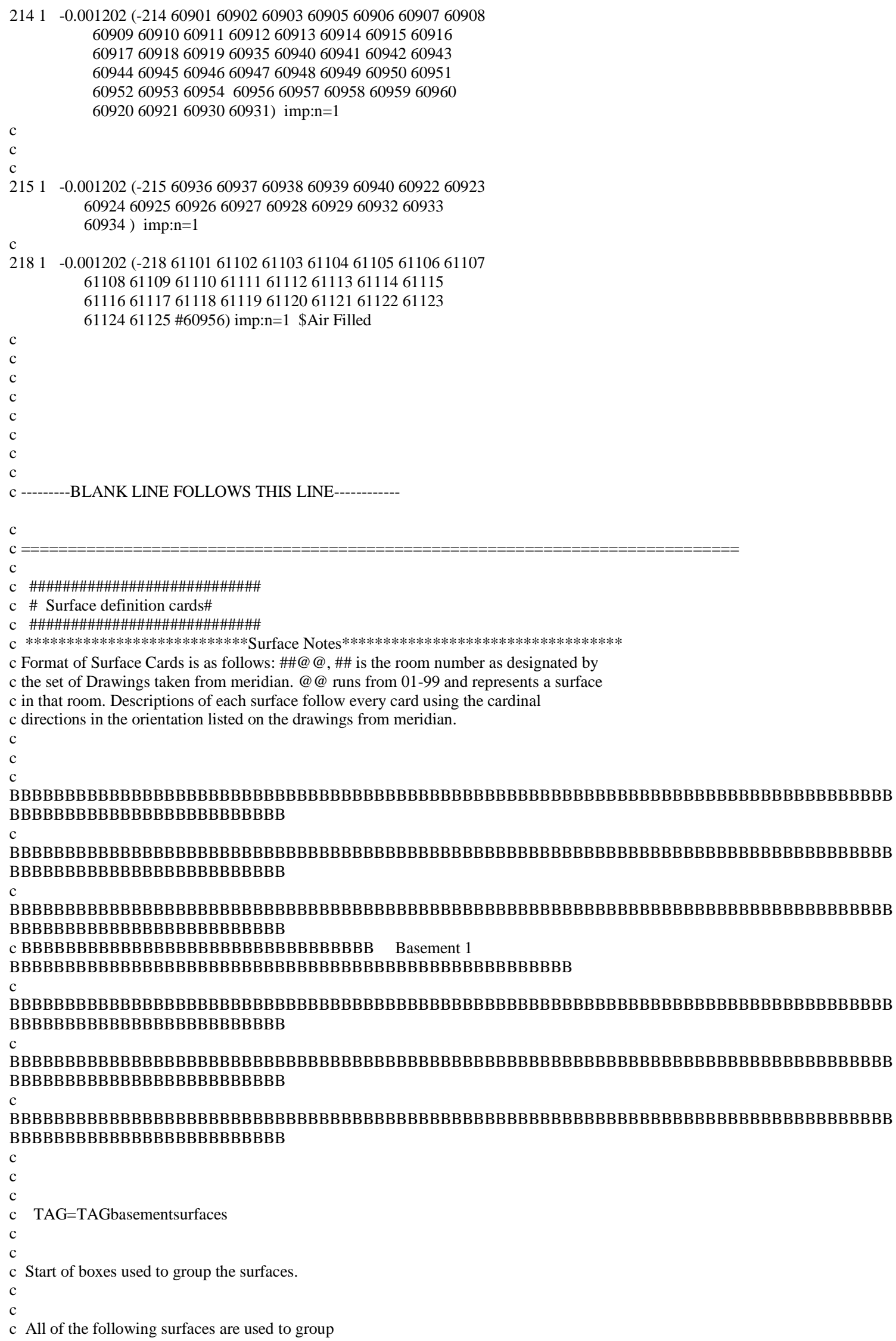


c the cells together. This had to be done to avoid overloading the data type associated with c cell cards. These boxes are purely for the codes sake and in no way affect the actual geometry c of the model.

c

999 rpp $\quad-1200 \quad 4000 \quad-3000 \quad 4000 \quad-304.8$ 1498.6 \$Void

c

c

c Void box surrounding model

990 rpp $\quad-1200 \quad 4000 \quad-3000 \quad 4000 \quad-304.8442 \quad$ \$Void

c

C

c NW Section of Basement 1

40146 rpp $-4.5 \quad 817 \quad-290 \quad 1091.5 \quad 0 \quad 432.5$ \$Cell box NW Section

C

C

c Begin North-Center Section of the Central Section of Basement1

40245 rpp $-48 \quad 526 \quad-96 \quad 549.5 \quad 0 \quad 432.5$ \$Cell box W

40345 rpp $526 \quad 998 \quad-96 \quad 621.5 \quad 0 \quad 432.5$ \$Cell box E

c

c Begin Northeast Section of the Central Section of Basement1

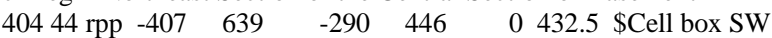

40544 rpp $639 \quad 1123.5 \quad-290 \quad 1091.5 \quad 0 \quad 432.5$ \$Cell box E

c

c Begin West Section of Basement1

40641 rpp $-179242 \quad 0 \quad 1152 \quad 0432.5$ \$Cell box W Section

c

c Begin Southwest Section of the Central Section of Basement1

407 rpp $-10 \quad 1187.5 \quad-10 \quad 998.5 \quad 0 \quad 432.5$ \$Cell box S Section

c

c Begin Southeast Section of the Central Section of Basement1

40842 rpp $-41 \quad 1123.5 \quad-15.5 \quad 993 \quad 0 \quad 432.5$ \$Section Cell box

C

c Begin East Section of Basement1

40943 rpp $-10 \quad 1083 \quad-10 \quad 1600 \quad 0 \quad 432.5$ \$Section Cell box

c

c Begin South Section of Basement1

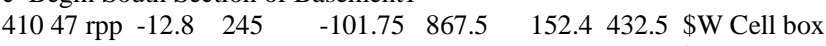

41147 rpp $245 \quad 1080 \quad-101.75 \quad 200 \quad 152.4432 .5$ \$S Cell box

41247 rpp $1080 \quad 1335.2 \quad-101.75 \quad 867.5 \quad 152.4 \quad 432.5$ \$E Cell box

41347 rpp $245 \quad 1080 \quad 200 \quad 867.5 \quad 152.4432 .5$ \$Central Cell box

C

c Below are the Decks of Basement1 and the Sand surrounding the building

c The southern mezzanine section is set $5 \mathrm{ft}$ above the B1 Deck. This is an observed measured distance because

c I have found no blueprint that clearly defines this distance. There is a small gap between Rm23B and the

c top of Rm32 where there is no sand and just air. This gap was left for the sake of saving time. No conservancy

c is lost. There is no sand up to the grade height in the SE corner of the building. This was done to be conservative.

c There are numerous areas in the SE that do not have a backfill to the same grade as the rest of the building.

c There is also a loading dock located in the SE corner which is simply free space.

$414 \quad$ rpp $-10 \quad 2390 \quad-10 \quad 2390 \quad-30.50 \quad$ \$Central Section of Basement foundation Deck

415 rpp $2390 \quad 2931.5 \quad-10 \quad 1551.5 \quad-30.50 \quad$ \$East Section of B1 foundation Deck

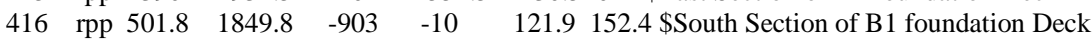

$417 \quad$ rpp $-264 \quad-10 \quad 121 \quad 538.5 \quad-30.50 \quad$ \$SW Bl foundation Deck

418 rpp $-431 \quad-10 \quad 538.5 \quad 1186.5 \quad-30.50 \quad$ \$W B1 foundation Deck

419 rpp $-1000 \quad 4000 \quad-2000 \quad 4000 \quad-304.8$-30.5 \$Sand 10ft thick under building

420 rpp $501.8 \quad 1849.8 \quad-903 \quad-10 \quad-30.5 \quad 121.9$ \$Sand filling the gap below South Section of B1

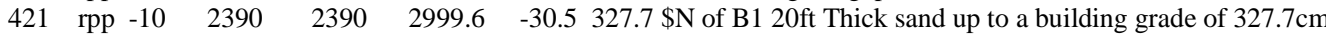

422 rpp $2390 \quad 2999.6 \quad 1551.5 \quad 2999.6 \quad-30.5 \quad 327.7$ \$NE of B1 20ft Thick sand up to a building grade of $327.7 \mathrm{~cm}$

423 rpp $501.8 \quad 1849.8 \quad-1512.6 \quad-903 \quad-30.5 \quad 327.7$ \$S of B1 20ft Thick sand up to a building grade of $327.7 \mathrm{~cm}$

$424 \quad$ rpp $-619.6501 .8 \quad-1512.6-10 \quad-30.5 \quad 327.7$ \$S2 20ft Thick sand up to a building grade of 327.7 cm

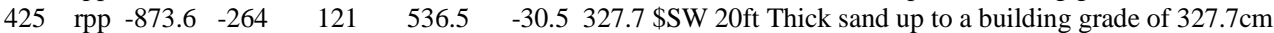

$426 \quad$ rpp $-1040.6-431 \quad 536.5 \quad 1186.5 \quad-30.5 \quad 327.7$ \$W 20ft Thick sand up to a building grade of $327.7 \mathrm{~cm}$

$427 \quad$ rpp $-609.6 \quad-10 \quad-10 \quad 121 \quad-30.5327 .7$ \$SW B1 20ft Thick sand up to a building grade of $327.7 \mathrm{~cm}$

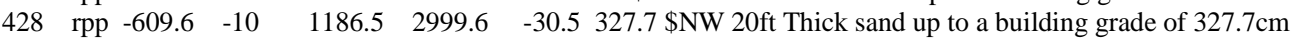




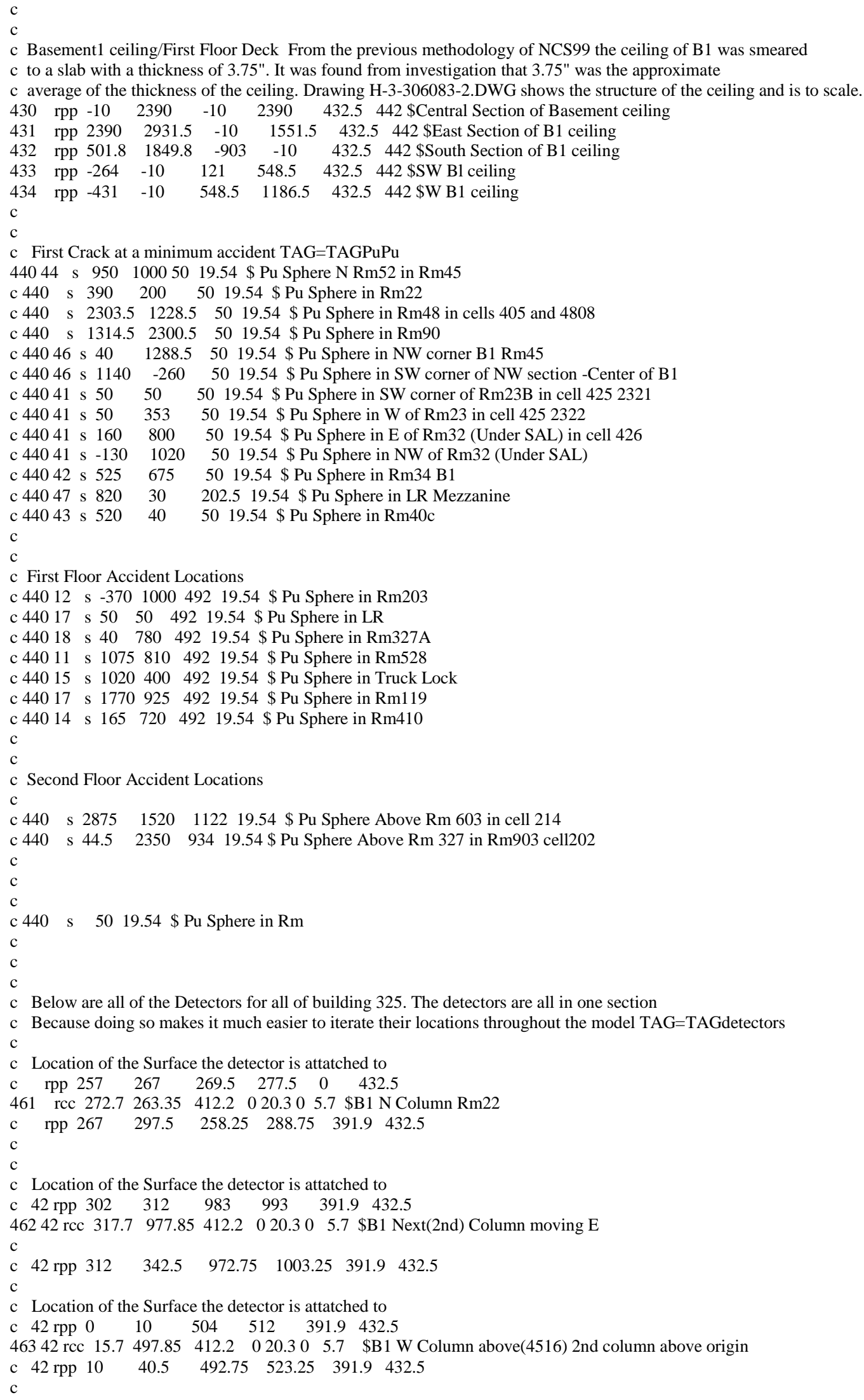


c Location of the Surface the detector is attatched to

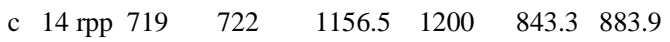

46414 rcc 713.31174 .65863 .6020 .305 .7 \$F1 W wl Rm516 Placed at the NW corner

c 46414 s 708.31174 .65863 .68 .66

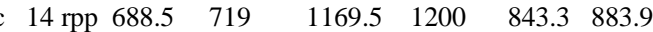

Location of the Surface the detector is attatched to

c 17 rpp $1252 \quad 1422 \quad 1050.75 \quad 1053.75 \quad 843.3 \quad 883.9$

46517 rcc 1315.25 1059.45 863.6 20.3 005.7 \$F1 N wl Rm115 Placed Approx. where observed

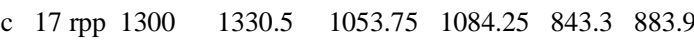

c Location of the Surface the detector is attatched to

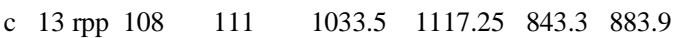

46613 rcc 102.31176 .5863 .6020 .305 .7 \$F1 W block wl Rm316

c 46613 s 97.31179 .5863 .68 .66

C

c 13 rpp $77.5 \quad 108 \quad 1086.75 \quad 1117.25 \quad 843.3 \quad 883.9$

Location of the Surface the detector is attatched to

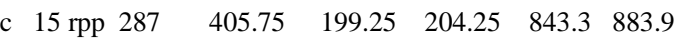

46715 rcc 390.5209 .95863 .620 .3005 .7 \$F1 N wl Rm605

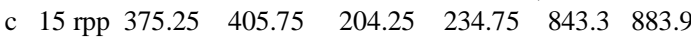

c Location of the Surface the detector is attatched to

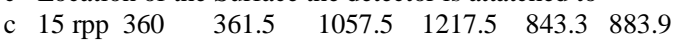

46815 rcc 367.2 1115.25 863.6 020.305 .7 \$F1 Central wl Rm603 Placed Approx. where observed

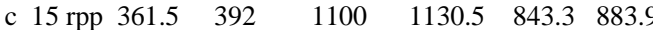

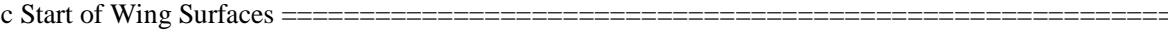

C

$\mathrm{C}$

c //I/I/I/I/////Begin Northwest Section of the Central Section of Basement1 IIIIIIIIIIIIIIIIIIIIIII

С $* * * * * * * * * * * * * * * * * *$ Notes for this section of Surfaces $* * * * * * * * * * * * * * * * * * * * * * * * * * * * * * * * * * * * * * * * * *$

c The origin $(0,0,0)$ was designated as the south west corner of the south westernmost pillar of

c the exploded basement drawing H-3-305009-1.DWG. This pillar is not under the yellow dashed line

c because these pillars were included in the south west Section model. Main structural wall was

c left out from this section to be included in a later model of the central basement area

c Basement1 Room 45

c This room contains mainly columns and is a continuation of Room45 in both the

c Southwest and Southeast sections. Room 45 is the number given to the largest room/open

c space of Basement1. Immeadiatly following this comment block will be the start of all of

c the columns for room 45. The first column will be the South Westernmost column from the exploded

c basement drawing. The numbering will go east from this column then return to the western edge

c once a terminating surface is encounterd. The numbering will then continue with the closest northern column.

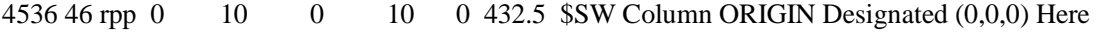

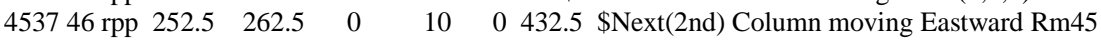

453846 rpp $505 \quad 515 \quad 0 \quad 10 \quad 0 \quad 432.5$ \$3rd Column moving East Rm45

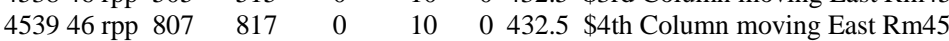

454046 rpp $1109 \quad 1119 \quad 0 \quad 10 \quad 0 \quad 432.5$ \$5th Column moving East Rm45 Farthest SE Column

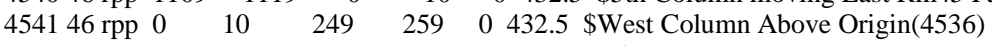

454246 rpp $252.5 \quad 262.5 \quad 249 \quad 259 \quad 0 \quad 432.5$ \$Next(2nd) Column moving Eastward Rm45

454346 rpp $505 \quad 515 \quad 249 \quad 259 \quad 0 \quad 432.5$ \$3rd Column moving East Rm45 


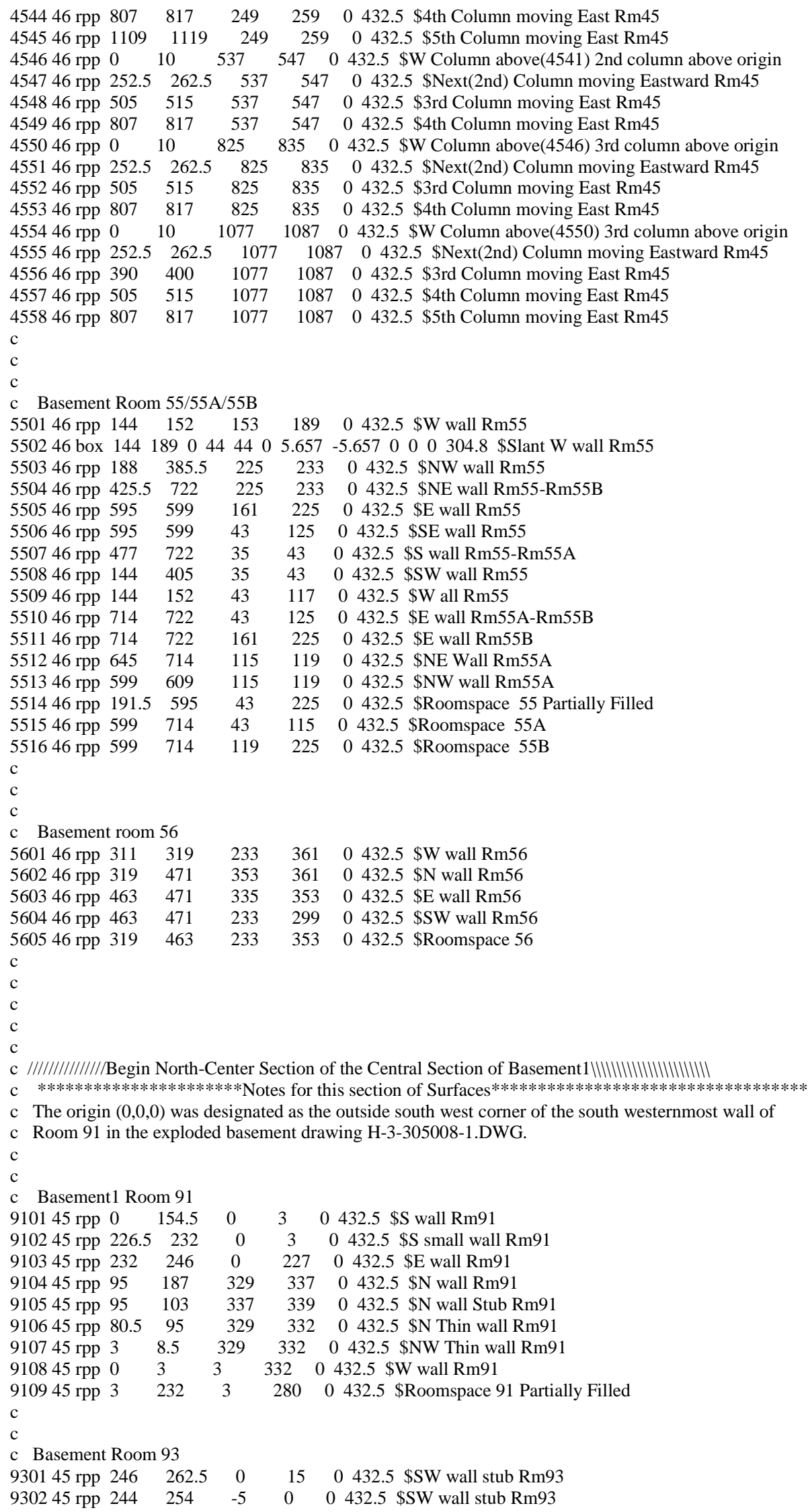




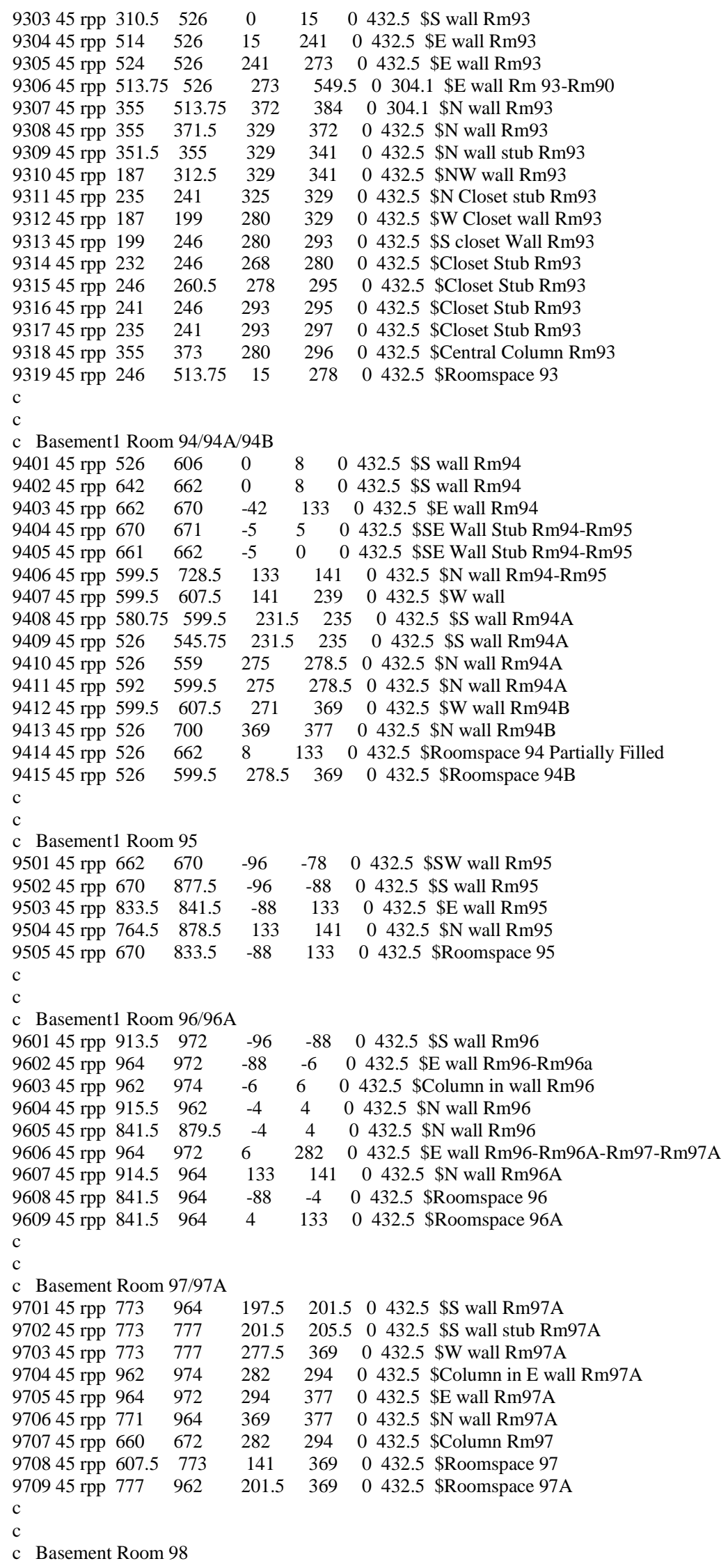




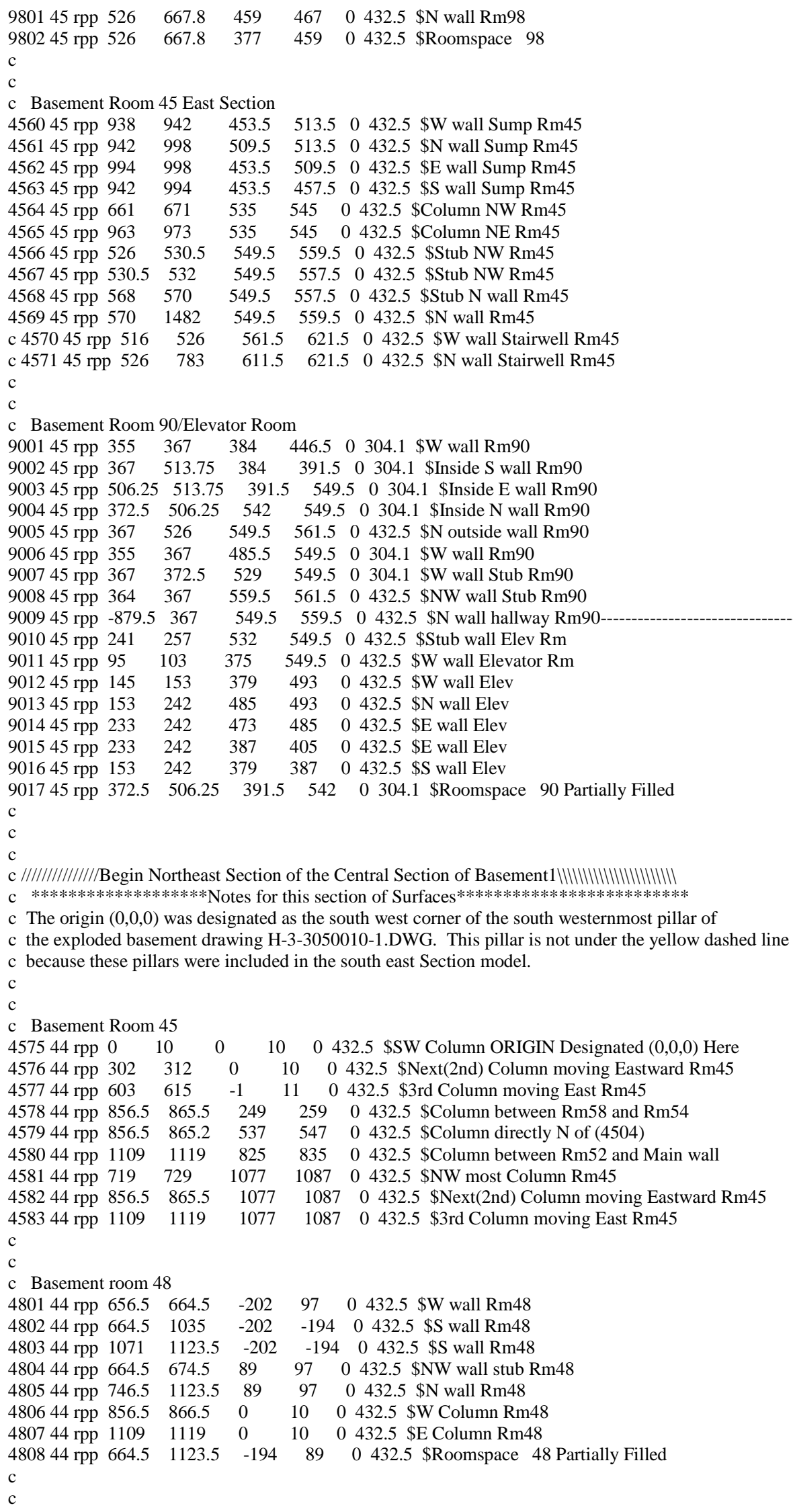




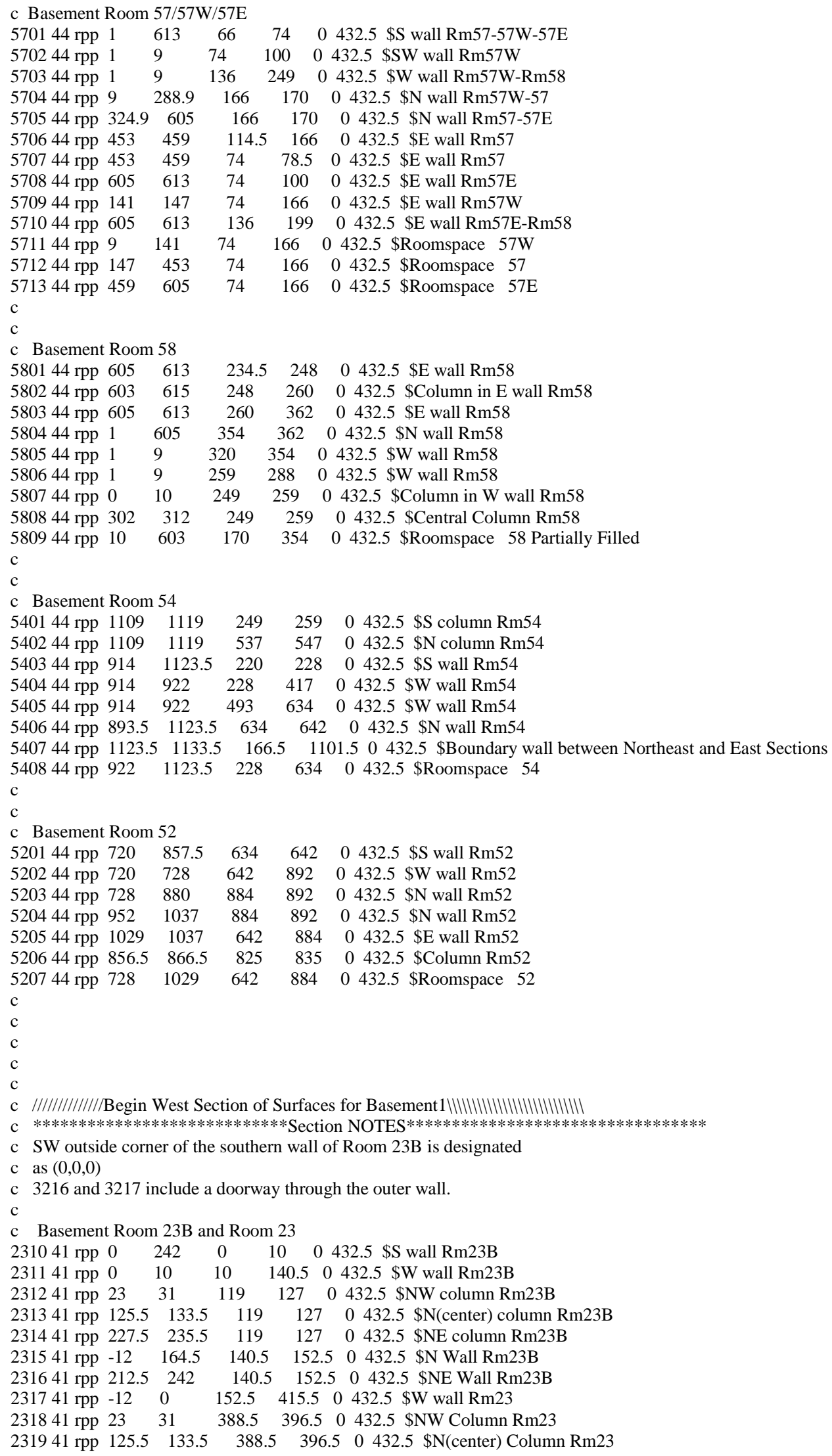




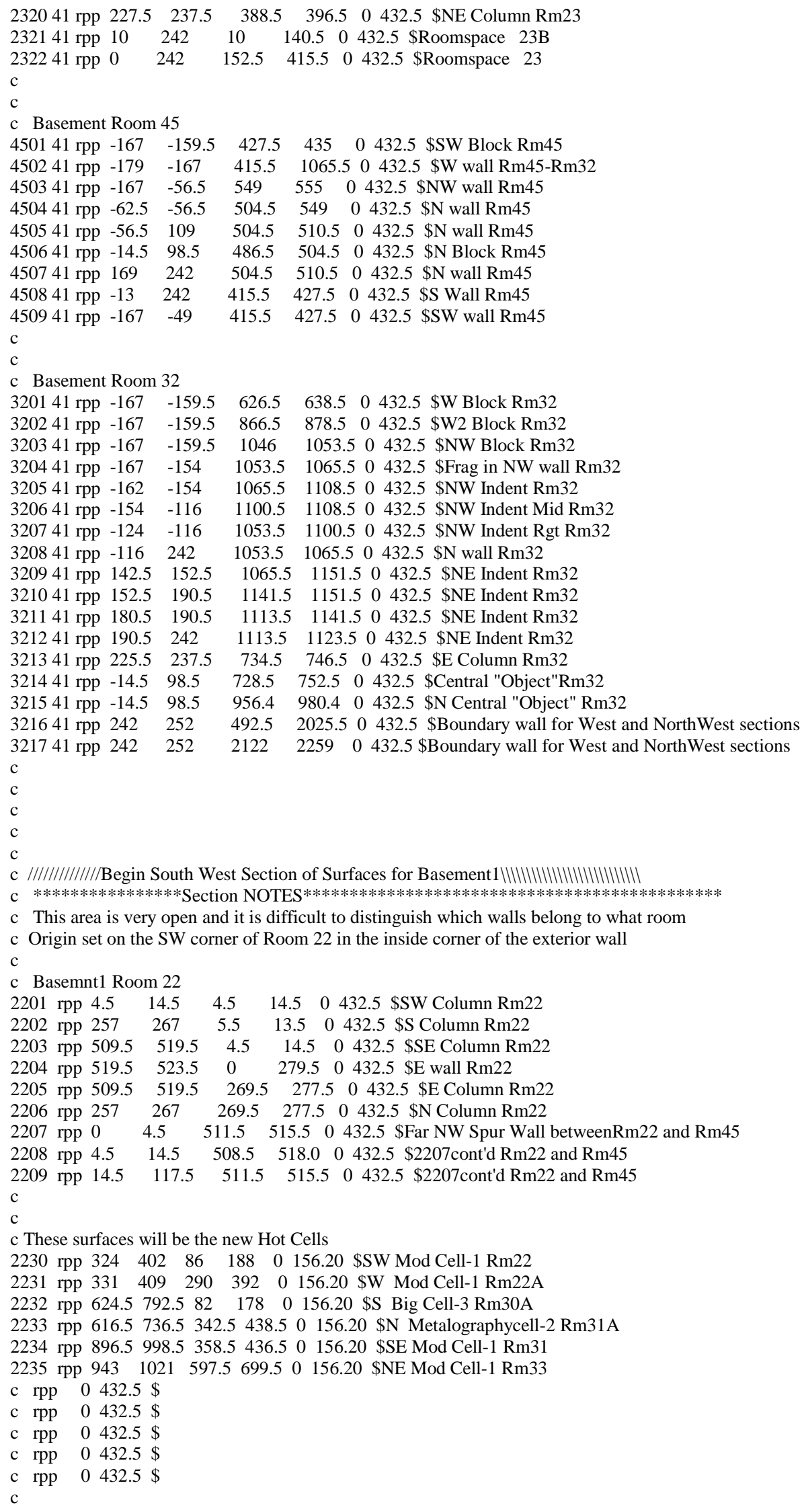




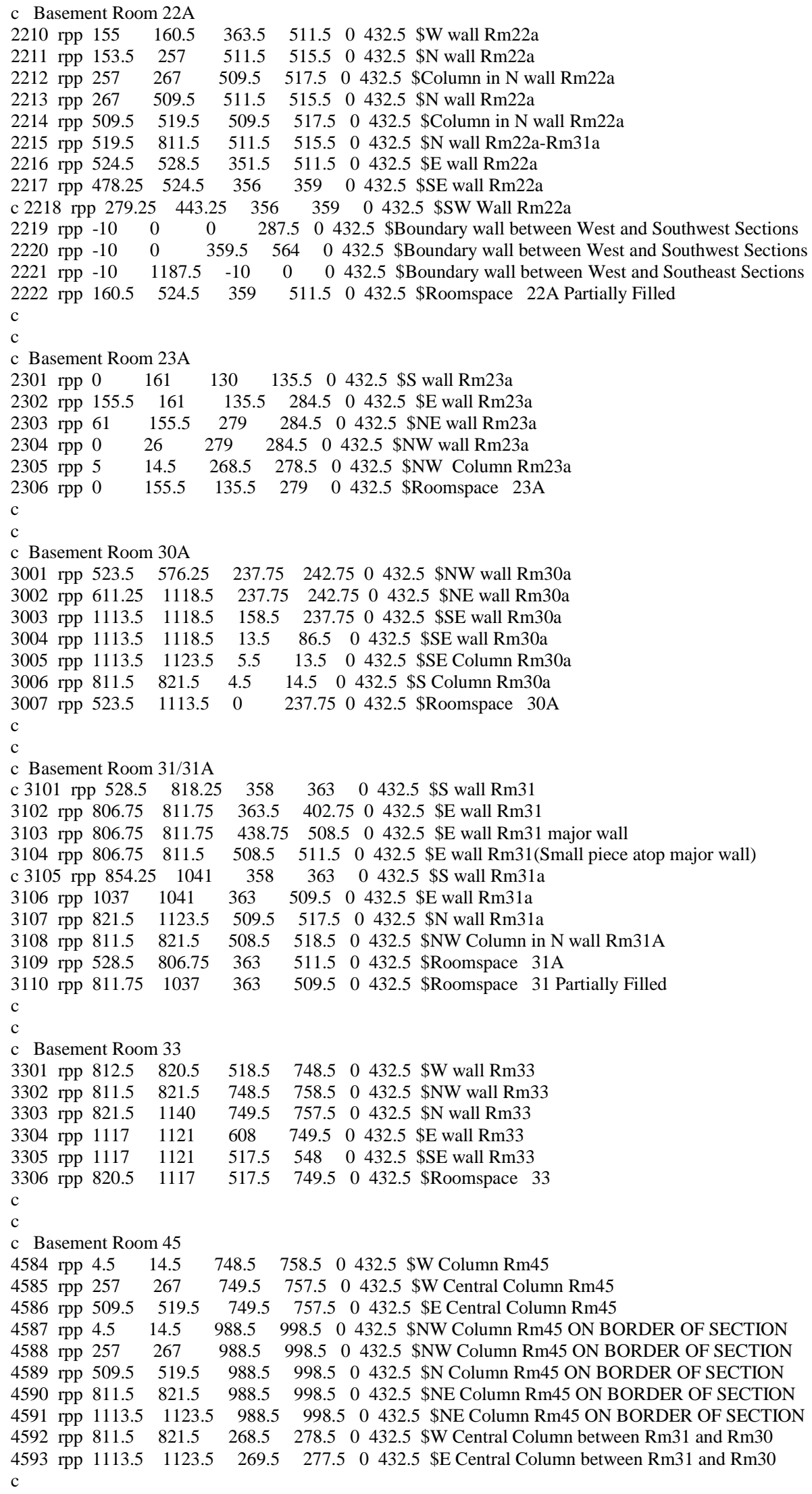




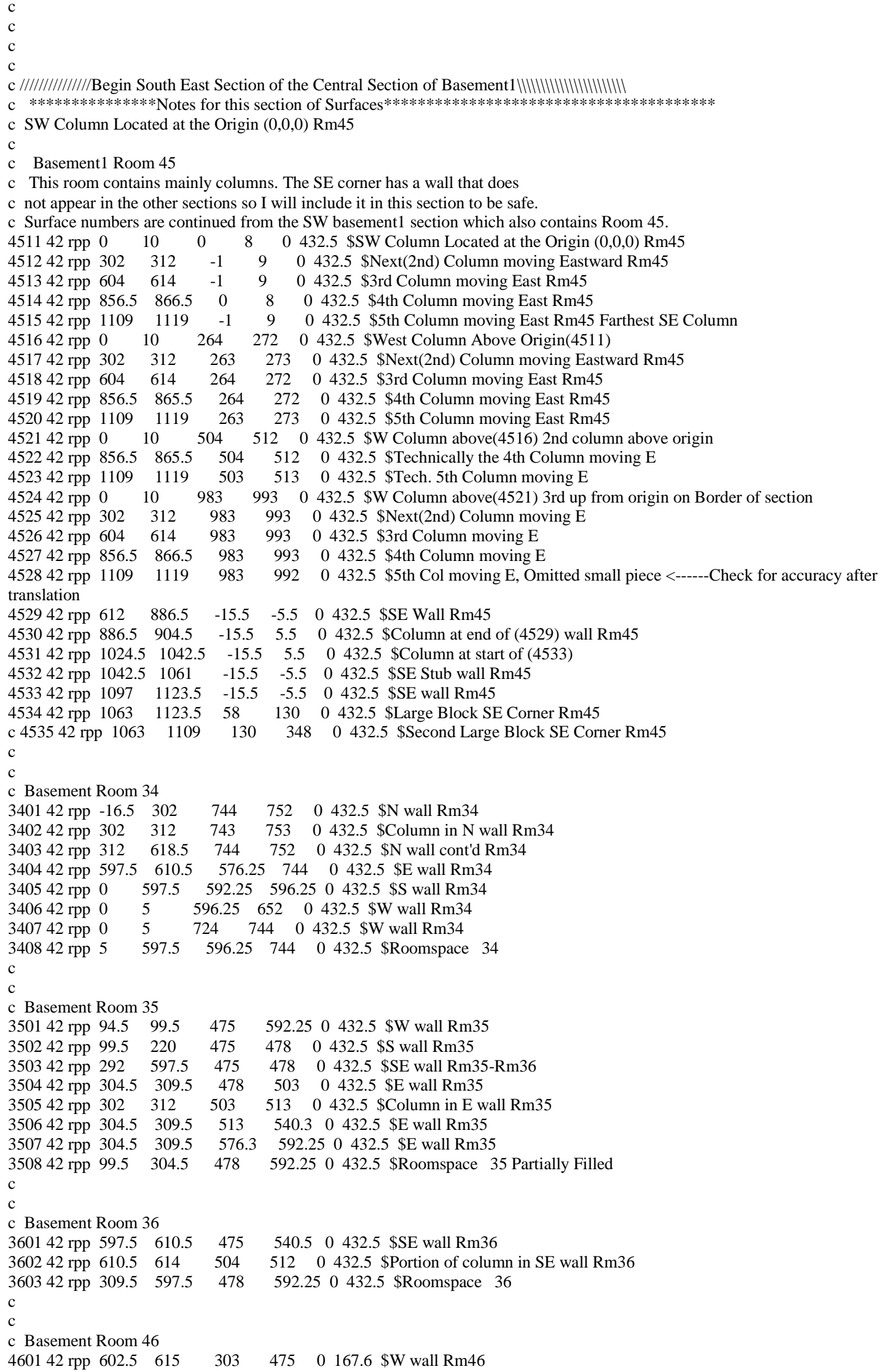




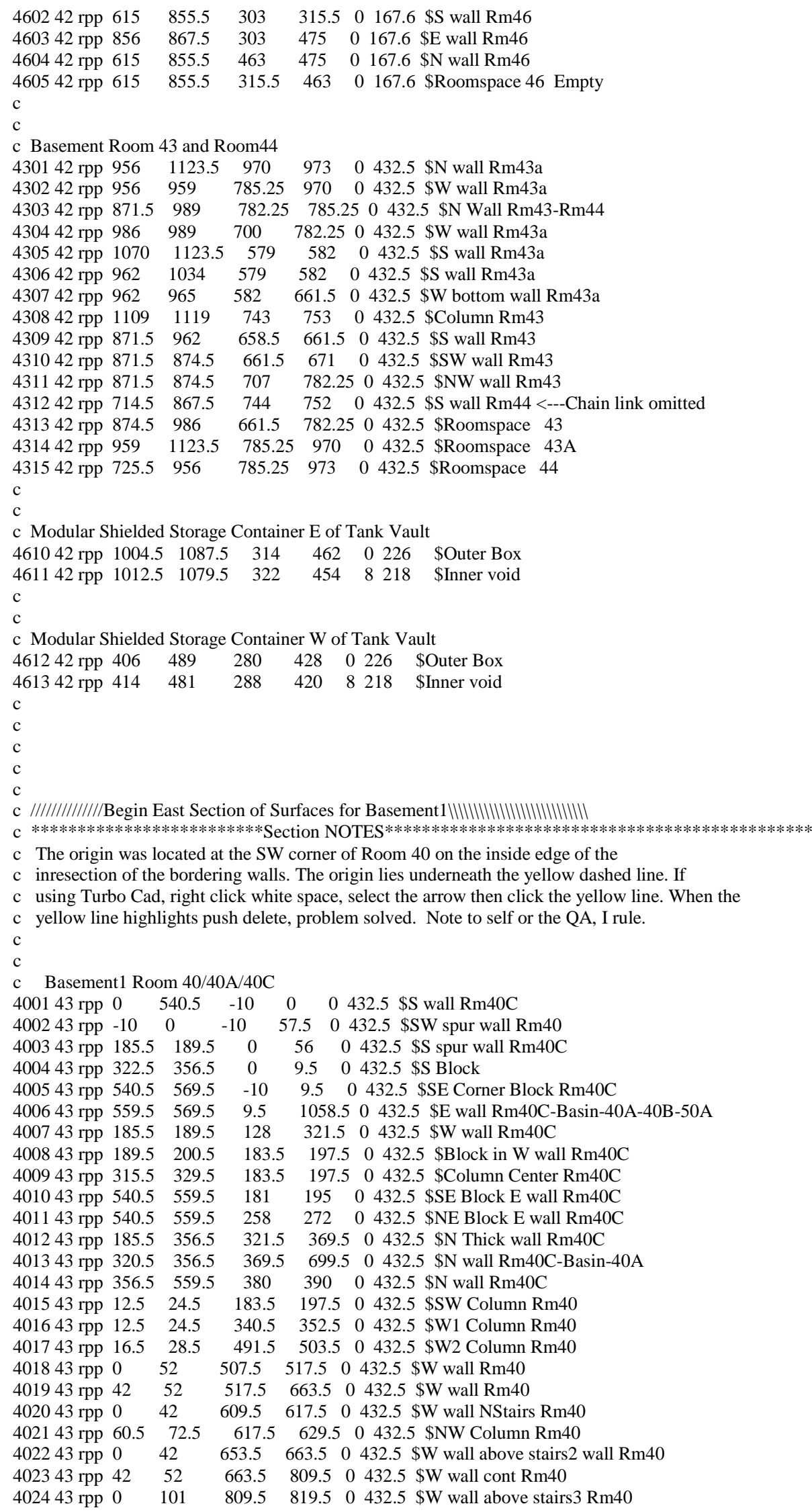




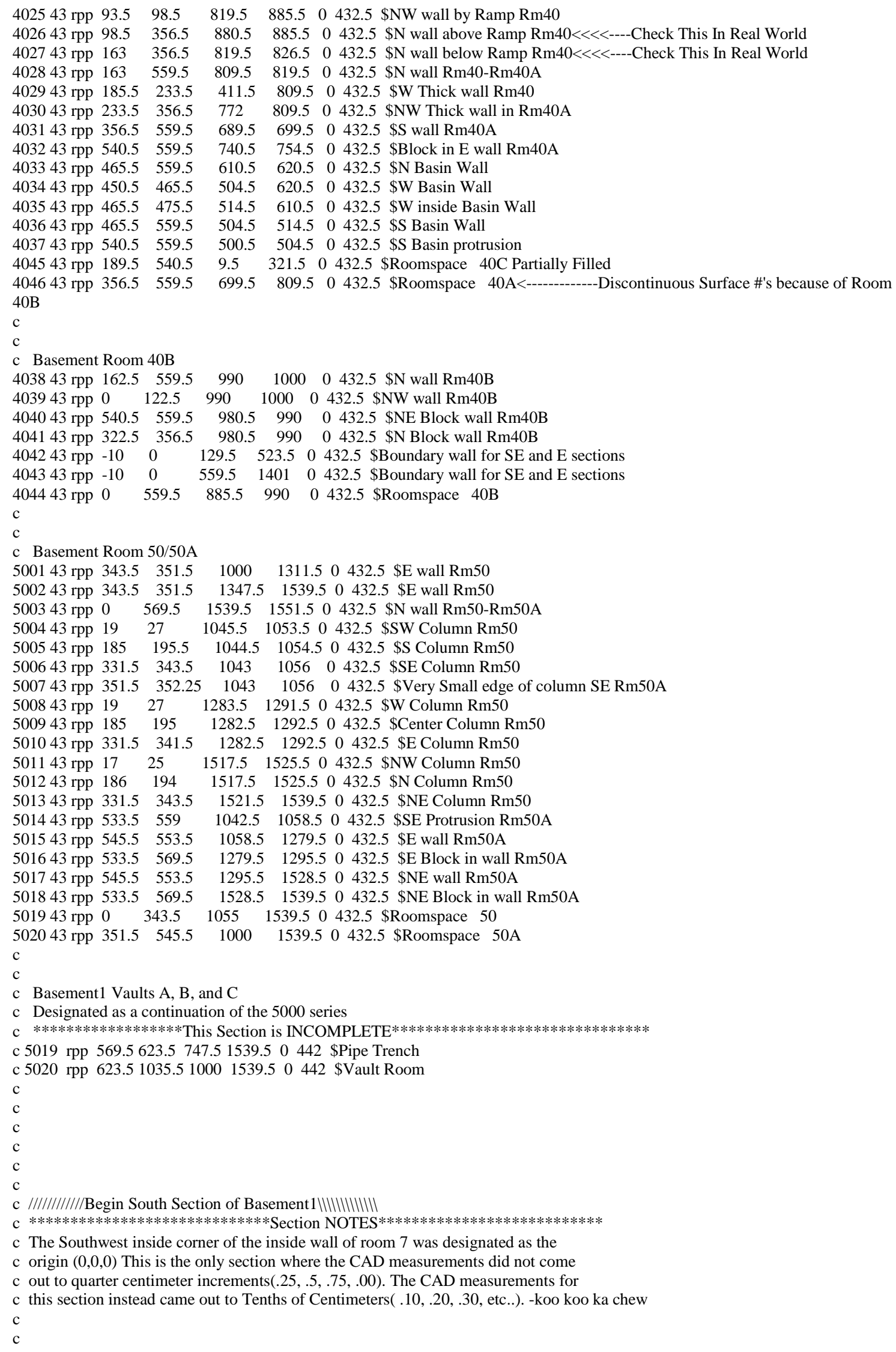




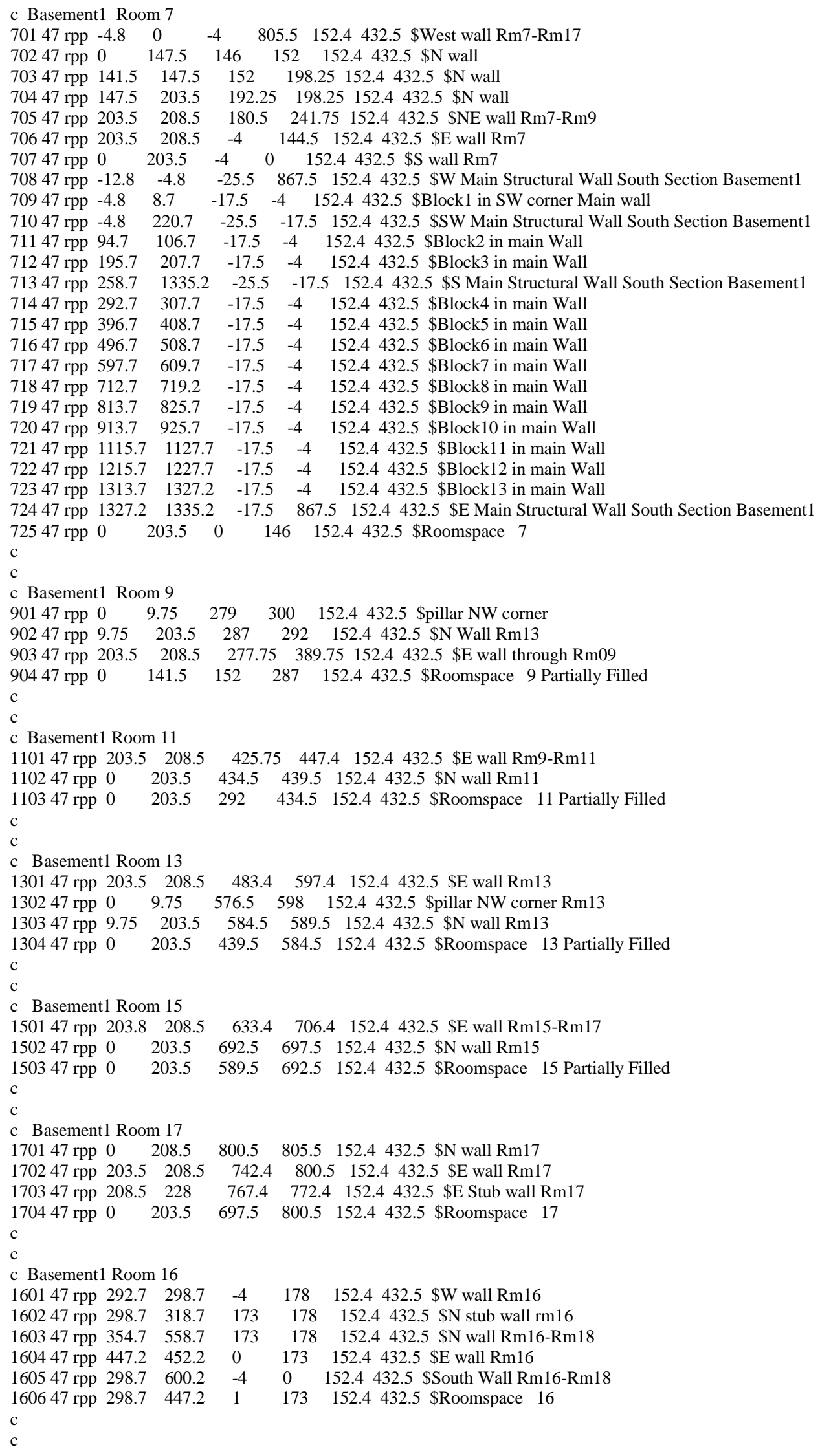




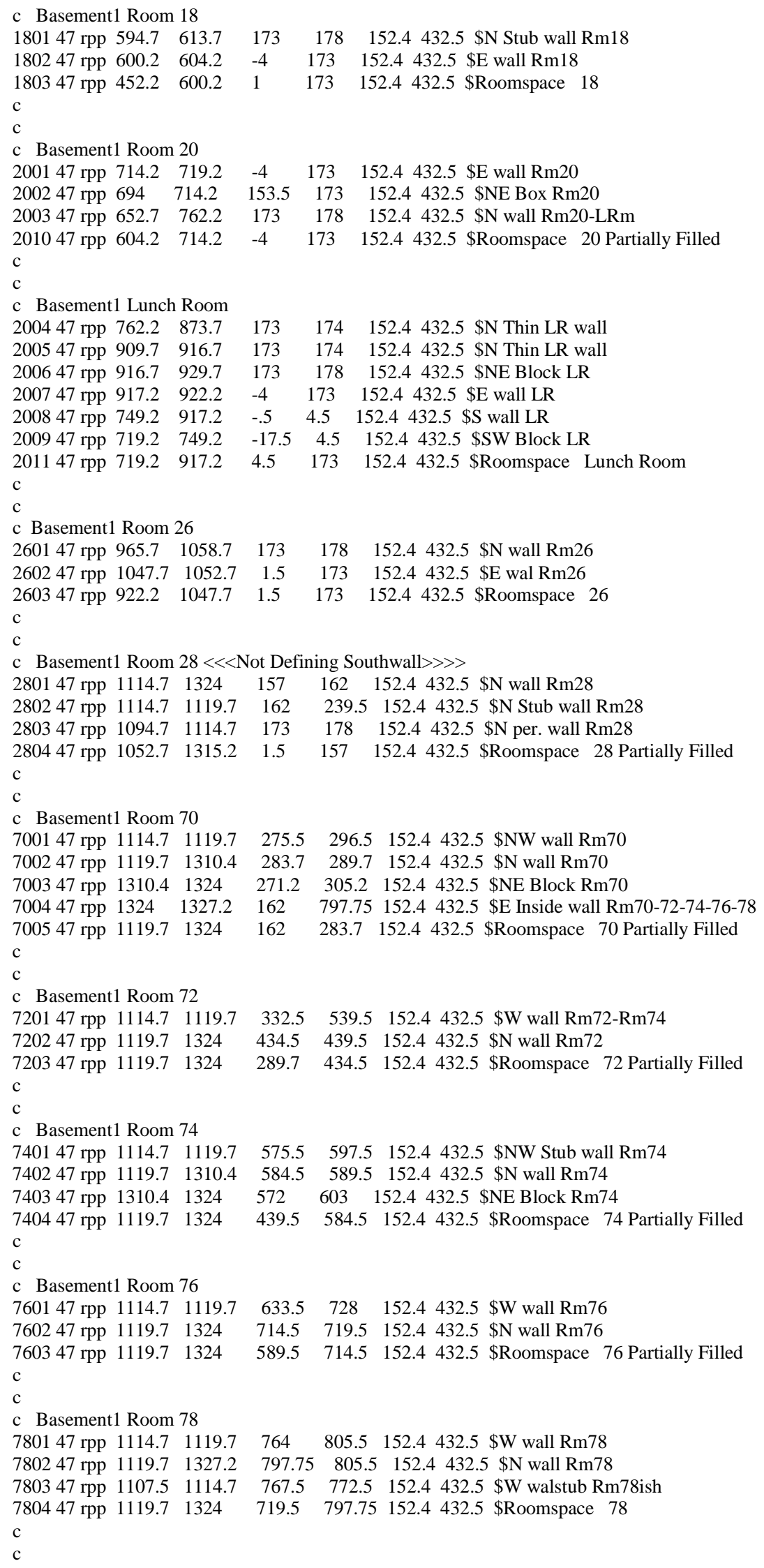




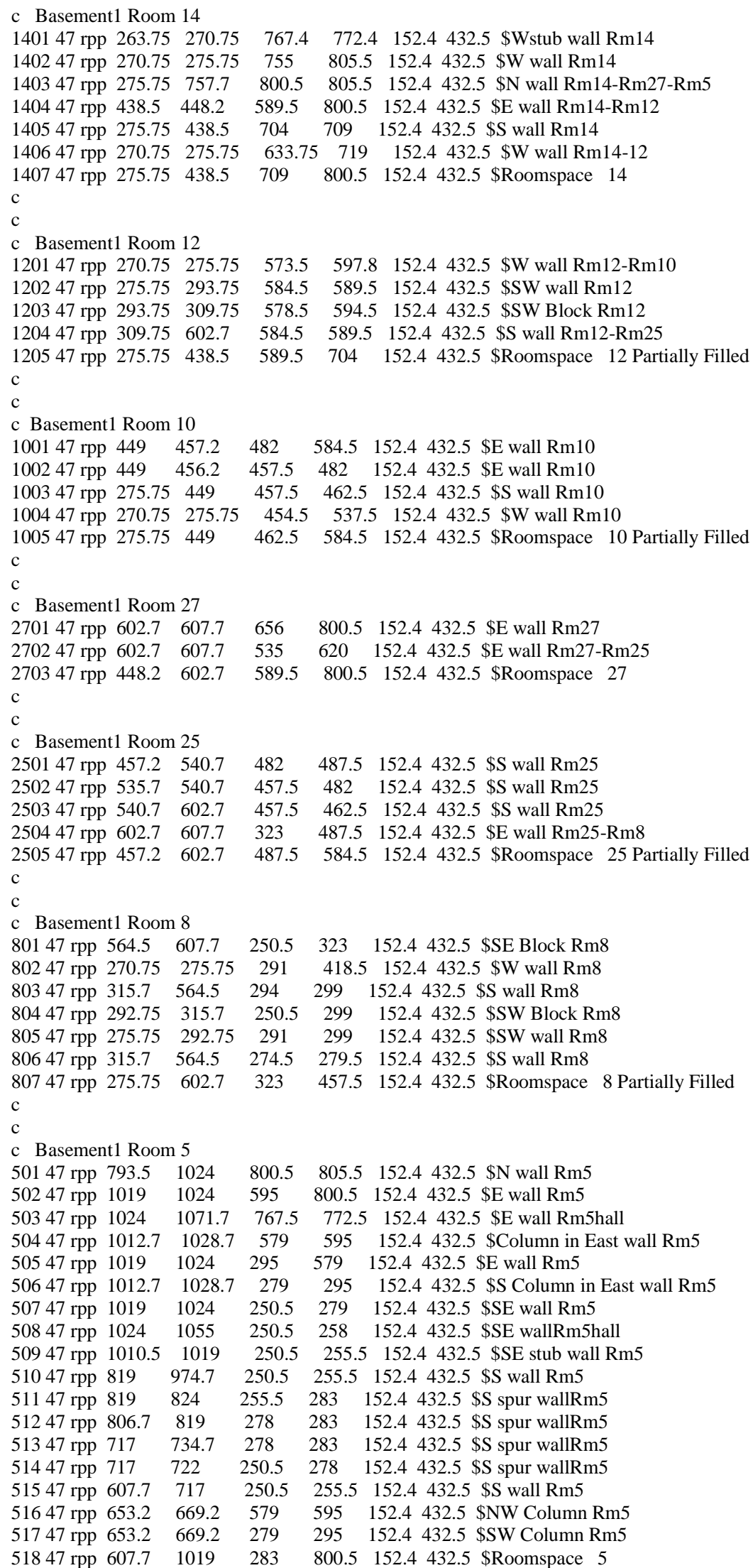




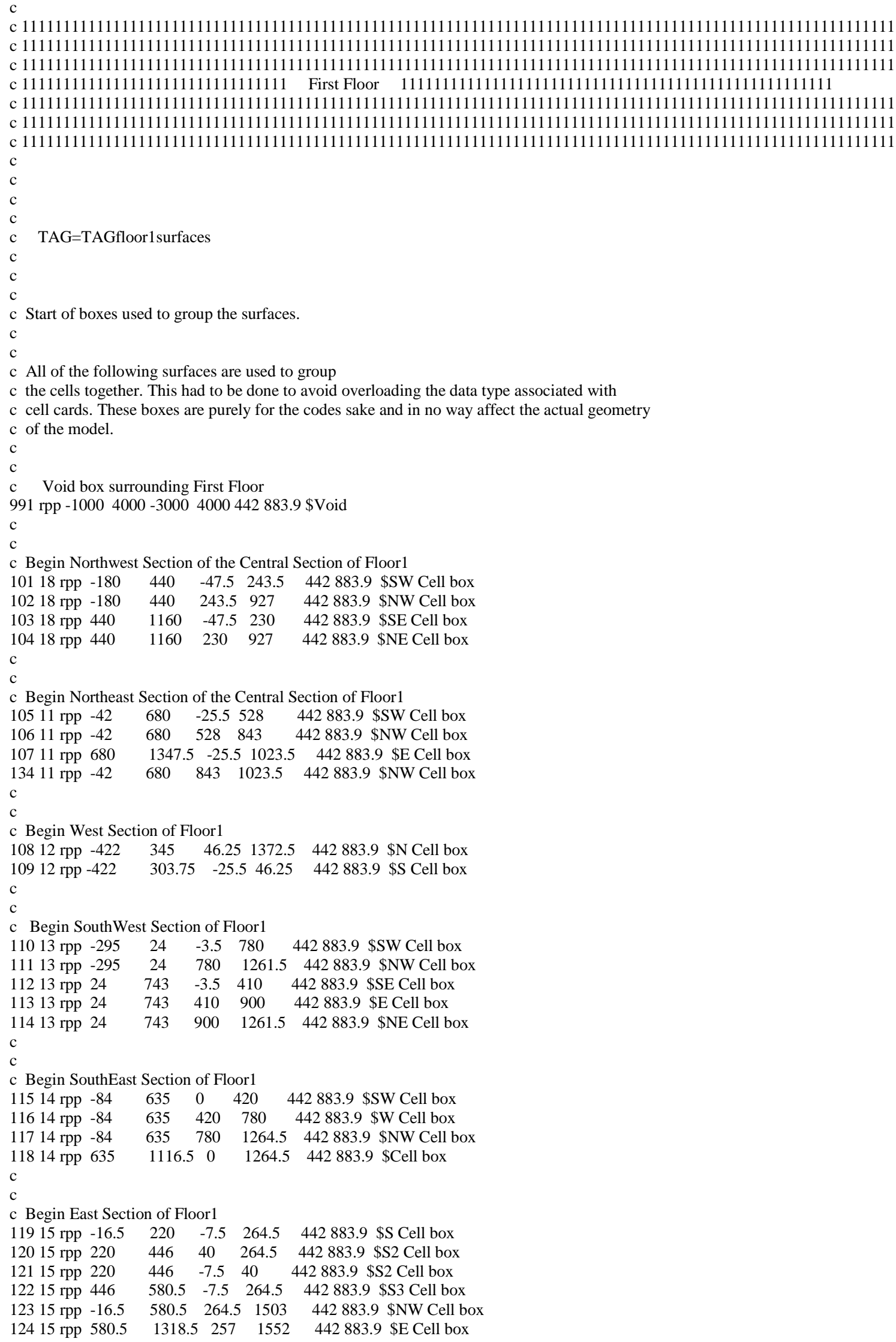




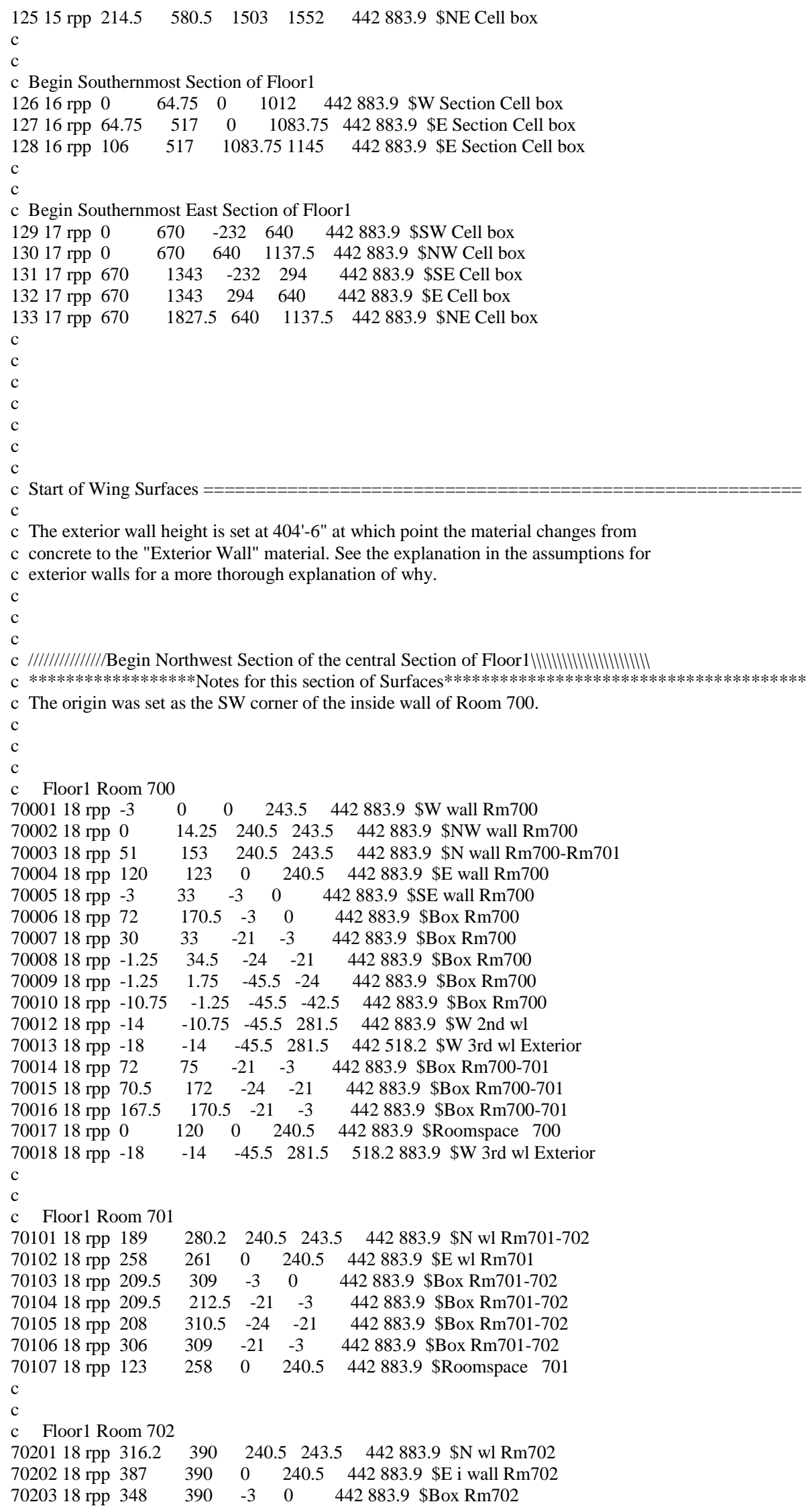


7020418 rpp $396.75 \quad 399.75 \quad-24 \quad 386.75 \quad 442$ 883.9 \$Eo wl Rm702

7020518 rpp $348 \quad 351 \quad-21 \quad-3 \quad 442883.9$ \$Box Rm702

7020618 rpp $346.5 \quad 396.75 \quad-24 \quad-21 \quad 442883.9$ \$Box Rm702

7020718 rpp $261 \quad 387 \quad 0 \quad 240.5 \quad 442883.9$ \$Roomspace 702

c

c Floor1 Room 703/Mens Bathroom/Unlabled Room Next to it

7030118 rpp $486.75 \quad 504.75 \quad-20.5 \quad-5.5 \quad 442883.9$ \$Box i air Rm703

7030218 rpp $483.75 \quad 507.75 \quad-23.5 \quad-2.5 \quad 442883.9$ \$Box o wl Rm703

7030318 rpp $483.75 \quad 486.75 \quad-2.5 \quad 150.75 \quad 442883.9$ \$W wl Rm703

$\begin{array}{llllll}70304 & 18 \text { rpp } 486.75 & 981.75 & 112 & 115 & 442883.9\end{array}$ \$N wl

$\begin{array}{llllll}70305 & 18 \text { rpp } 507.75 & 569.75 & -23.5 & -20.5 & 442883.9 \\ \text { \$SE wl }\end{array}$

$\begin{array}{llllll}70306 & 18 \text { rpp } 604.75 & 821.75 & -23.5 & -20.5 & 442883.9\end{array}$ \$S wl

7030718 rpp $856.75 \quad 995.75 \quad-23.5 \quad-20.5 \quad 442883.9$ \$S wl Mens Rm

7030818 rpp $1030.25 \quad 1118.75$-23.5 $-20.5 \quad 442$ 883.9 \$SE wl Mens Rm

7030918 rpp $861.75 \quad 864.75 \quad-20.5 \quad 112 \quad 442883.9$ \$W wl Mens Rm

7031018 rpp $981.75 \quad 989.75 \quad-20.5 \quad 27 \quad 442883.9$ \$Divider wl Mens Rm

7031118 rpp $981.75 \quad 989.75 \quad 63 \quad 103 \quad 442883.9$ \$Divider wl Mens Rm

7031218 rpp $981.751094 .75103 \quad 115 \quad 442883.9$ \$N Block Mens Rm

7031318 rpp $1034.25 \quad 1094.75$-20.5 $-15.5 \quad 442$ 883.9 \$S Block Mens Rm

7031418 rpp $1094.75 \quad 1097.75$-20.5 $174.25 \quad 442$ 883.9 \$E wl Mens Rm

7031518 rpp $1115.75 \quad 1118.75$-20.5 $133.75 \quad 442$ 883.9 \$E o wl Mens Rm

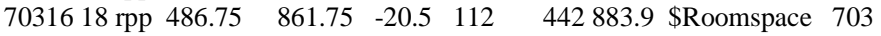

c

c $\quad$ Floor1 Room 320/421

3200118 rpp $504.75 \quad 507.75 \quad 115 \quad 211 \quad 442883.9$ \$W box Rm320

$\begin{array}{llllll}32002 & 18 \text { rpp } 486.75 & 504.75 & 208 & 211 & 442883.9\end{array}$ \$W box Rm320

3200318 rpp $483.75 \quad 486.75 \quad 178 \quad 212.5 \quad 442883.9$ \$W box Rm320

3200418 rpp $483.75 \quad 486.75248 .5445 \quad 442883.9$ \$NW box Rm320-324

3200518 rpp $486.75 \quad 507.75 \quad 250 \quad 253 \quad 442$ 883.9 \$NW box Rm320

3200618 rpp $504.75 \quad 507.75253 \quad 511 \quad 442883.9$ \$NW box Rm320

3200718 rpp $486.75 \quad 504.75508 \quad 511 \quad 442883.9$ \$NW box Rm320

3200818 rpp $483.75 \quad 486.75 \quad 481 \quad 512.5 \quad 442883.9$ \$NW box Rm320

3200918 rpp $507.75 \quad 1094.75350 .5 \quad 353.5 \quad 442883.9$ \$NW wl Rm320

3201018 rpp $1094.75 \quad 1097.75250 .25510 .25 \quad 442$ 883.9 \$E box Rm320

3201118 rpp $1097.75 \quad 1118.75 \quad 507.25510 .25 \quad 442$ 883.9 \$E box Rm320

3201218 rpp $1115.75 \quad 1118.75 \quad 369.25 \quad 507.25 \quad 442$ 883.9 \$E box Rm320

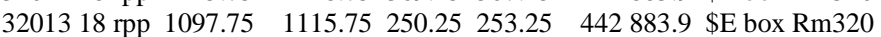

3201418 rpp $1115.75 \quad 1118.75248 .25 \quad 339.25 \quad 442883.9$ \$E box Rm320

3201518 rpp $1097.75 \quad 1115.75 \quad 171.25 \quad 174.25 \quad 442883.9$ \$E box Rm320

3201618 rpp $1115.75 \quad 1118.75160 .75 \quad 176.25 \quad 442883.9$ \$E box Rm320

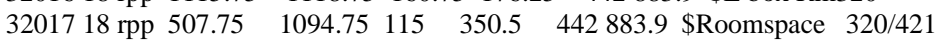

C

C

c Floor1 Room 324/425

3240118 rpp $486.75 \quad 504.75553 \quad 627.5 \quad 442883.9$ \$W i box air filled

3240218 rpp $483.75 \quad 507.75 \quad 550 \quad 630.5 \quad 442883.9$ \$W o box Conc filled

$\begin{array}{llllll}32403 & 18 \text { rpp } 486.75 & 504.75 & 672.5 & 742.5 & 442883.9\end{array}$ \$NW i box air filled

3240418 rpp $483.75 \quad 507.75 \quad 669.5 \quad 745.5 \quad 442883.9$ \$NW o box Conc filled

3240518 rpp $507.75 \quad 749.75 \quad 701.5 \quad 704.5 \quad 442883.9$ \$N wl Rm324

3240618 rpp $749.75 \quad 752.75 \quad 569 \quad 742.5 \quad 442883.9$ \$NE wl

3240718 rpp $752.75 \quad 806 \quad 542.5 \quad 566 \quad 442883.9$ \$NE i box air filled

3240818 rpp $749.75 \quad 809 \quad 539.5 \quad 569 \quad 442883.9$ \$NE o box Conc filled

3240918 rpp 847.25 $949.25 \quad 566 \quad 569 \quad 442883.9$ \$NE wl

3241018 rpp $946.25 \quad 949.25 \quad 569 \quad 606 \quad 442883.9$ \$NE wl

3241118 rpp $949.25 \quad 1027.25603 \quad 606 \quad 442883.9$ \$NE wl

3241218 rpp $1024.25 \quad 1027.25561 .75 \quad 603 \quad 442$ 883.9 \$NE wl

3241318 rpp $1027.25 \quad 1093.75561 .75564 .75 \quad 442883.9$ \$NE wl

3241418 rpp $1096.751116 .75553 .75571 .75 \quad 442$ 883.9 $\$$ \$NE i box air filled

3241518 rpp $1093.75 \quad 1119.75550 .75574 .75 \quad 442883.9$ \$NE o box Conc filled

3241618 rpp $1115.75 \quad 1118.75 \quad 546.25 \quad 550.75 \quad 442$ 883.9 $\$$ \$E Stub wl

3241718 rpp $1119.75 \quad 1142.75550 .75 \quad 553.75 \quad 442$ 883.9 \$E stub wl

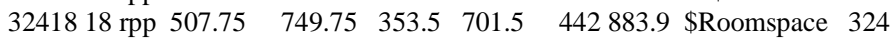

3241918 rpp $749.75 \quad 1094.75353 .5 \quad 539.5 \quad 442883.9$ \$Roomspace 425 Partially Filled

C

c Floor1 Room 330/Room 427/Elevator

c $* * * * *$ Small protrusions ignored in west and east hallways*****

c I also ommitted the strange caps on the E edge of the elevator because I was unable to 
c to verify they existed.

3300118 rpp 504.75

3300218 rpp 486.75

3300318 rрp 483.75

3300418 rpp 461.25

3300518 rpp 461.25

3300618 rpp 507.75

3300718 rpp 464.25

3300818 rpp 473.25

3300918 rpp 749.75

3301018 rpp 752.75

3301118 rpp 845.75

3301218 rpp 946.25

3301318 rpp 947

3301418 rpp 955

3301518 rpp 955

3301618 rpp 997

3301718 rpp 1005

3301818 rpp 1005

$\begin{array}{lllll}507.75 & 784.5 & 821.5 & 442883.9 & \text { \$NW Block } \\ 504.75 & 784.5 & 787.5 & 442883.9 \$\end{array}$

$\begin{array}{lllll}486.75 & 783 & 813.5 & 442883.9 \$\end{array}$

$\begin{array}{lllll}483.75 & 810.5 & 813.5 & 442883.9 \$\end{array}$

$\begin{array}{lllll}464.25 & 813.5 & 824.75 & 442883.9 & \$\end{array}$

$\begin{array}{llll}947 & 818 & 821.5 & 442883.9\end{array} \mathrm{\$ N}$ i wl

$947 \quad 821.5 \quad 824.75 \quad 442883.9$ \$N wl 2nd wl moving north

$947 \quad 824.75836 \quad 442518.2$ \$N Exterior wl

$\begin{array}{llll}752.75 & 779.5 \quad 818 \quad 442883.9 \text { \$E wl Rm330 }\end{array}$

$848.75 \quad 808.5 \quad 811.5 \quad 442883.9$ \$Extension wl into Rm427

$848.75811 .5 \quad 818 \quad 442883.9$ \$Extension wl into Rm427

$947 \quad 606 \quad 818 \quad 442883.9$ \$Very thin E wl Rm427

$955 \quad 606 \quad 829 \quad 442883.9$ \$W wl Elevator

$1141.25821 \quad 829 \quad 442518.2$ \$N o Elev wl

$1093.75606 \quad 614 \quad 442883.9$ \$S Elev wl

$1005 \quad 655.5 \quad 770.25 \quad 442883.9$ \$W Elev shaft

$1094 \quad 762.25 \quad 770.25 \quad 442883.9$ \$N Elev shaft

$1094 \quad 655.5 \quad 663.5 \quad 442883.9$ \$S Elev shaft

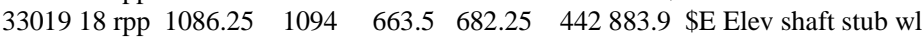

3302018 rpp 1086.25 $1094 \quad 750.25762 .25 \quad 442883.9$ \$E Elev shaft stub wl

3302118 rpp $1044.751050 .75564 .75606 \quad 442883.9$ \$SE compartment wl

3302218 rpp $1050.751093 .75564 .75570 .75 \quad 442883.9$ \$S compartment wl

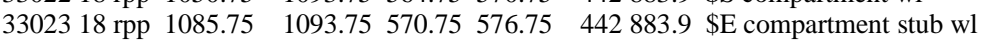

3302418 rpp $1085.75 \quad 1093.75602 \quad 606 \quad 442883.9$ \$E compartment stub wl

3302518 rpp $1093.75 \quad 1098.75574 .75616 .75 \quad 442883.9$ \$E compartment capping wl

3302618 rpp $1093.751141 .25815 .75821 \quad 442883.9$ \$Doodad in E hallway on N wl

3302718 rpp $1093.75 \quad 1111 \quad 809.75815 .75 \quad 442883.9$ \$Doodad in E hallway on N wl

3302818 rpp $1093.75 \quad 1098.75806 \quad 809.75 \quad 442883.9$ \$Doodad in E hallway on N wl

$\begin{array}{llllll}33029 & 18 \text { rpp } 507.75 & 749.75 & 704.5 & 818 & 442883.9\end{array}$ \$Roomspace 330

3303018 rpp $752.75 \quad 946.25 \quad 569 \quad 808.5 \quad 442883.9$ \$Roomspace 427 Partially Filled

3303118 rpp $955 \quad 1141.25821 \quad 829 \quad 518.2883 .9$ \$N o Elev wl

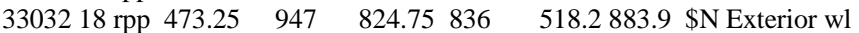

C

c Floor1 Room 325/Filter Building

C $* * * * * * * * * * * *$ Ignored small blocks on ramp*****************

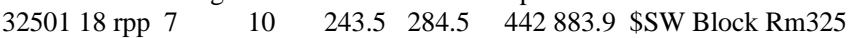

3250218 rpp $-14 \quad 7 \quad 281.5 \quad 284.5 \quad 442883.9$ \$

3250318 rpp $-3 \quad 0 \quad 359.5 \quad 546.5 \quad 442883.9$ \$W wl

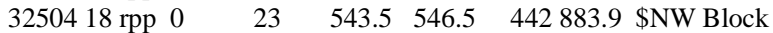

3250518 rpp $20 \quad 23 \quad 546.5 \quad 577.5 \quad 442883.9 \$$

3250618 rpp $-3 \quad 20 \quad 574.5 \quad 577.5 \quad 442883.9$ \$

3250718 rpp $-3 \quad 0 \quad 577.5 \quad 810 \quad 442883.9$ \$W wl Rm325-Rm327

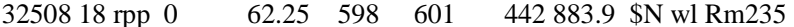

3250918 rpp $98.25 \quad 375.75 \quad 598 \quad 601 \quad 442883.9$ \$N wl Rm235

3251018 rpp $375.75 \quad 378.75556 .5 \quad 641 \quad 442883.9$ \$NE BlockRm235

3251118 rpp $378.75 \quad 396.75 \quad 638 \quad 641 \quad 442883.9$ \$NE BlockRm235

3251218 rpp $396.75 \quad 399.75613 .75642 .5 \quad 442883.9$ \$NE BlockRm235

3251318 rpp $396.75 \quad 399.75 \quad 555 \quad 577.5 \quad 442$ 883.9 \$NE BlockRm235

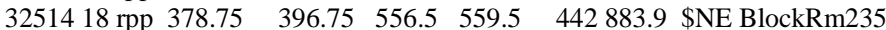

3251518 rpp $375.75 \quad 378.75 \quad 243.5 \quad 481.5 \quad 442883.9$ \$E wl Rm235

3251618 rpp $378.75 \quad 396.75 \quad 478.5 \quad 481.5 \quad 442883.9$ \$E Block

$\begin{array}{llllll}32517 & 18 \text { rpp } 396.75 & 399.75 & 459 & 483 & 442883.9 \text { \$ }\end{array}$

3251818 rpp $-6 \quad-3 \quad 362.5 \quad 811.5 \quad 442883.9$ \$W 2nd wl moving outward

3251918 rpp $-10.75 \quad-6 \quad 362.5 \quad 811.5 \quad 442883.9$ \$W 3rd wl moving outward

3252018 rpp $-14 \quad-10.75 \quad 362.5 \quad 824.75 \quad 442883.9$ \$W 4th wl moving outward

3252118 rpp $-18.25 \quad-14 \quad 362.5 \quad 829 \quad 442518.2$ \$W 5th wl moving outward

3252218 rpp $-147.25 \quad-32.25 \quad 585 \quad 693 \quad 442883.9$ \$Cover of Filter

3252318 rpp $10 \quad 375.75243 .5460 \quad 442883.9$ \$Roomspace 325 Partially Filled -Fix

This

3252418 rpp $-18.25 \quad-14 \quad 362.5 \quad 829 \quad 518.2883 .9$ \$W 5th wl moving outward

C

C Floor1 Room 327/327A

$\begin{array}{llllll}32701 & 18 \text { rpp } 237.75 & 240.75 & 601 & 638 & 442883.9\end{array}$ \$Dividing wl Rm327/327A

3270218 rpp $237.75 \quad 240.75674 \quad 818 \quad 442883.9$ \$Dividing wl Rm327/327A

$\begin{array}{llllll}32703 & 18 \text { rpp } 375.75 & 378.75 & 680 & 818 & 442 \\ 3 & 883.9 & \text { \$E wl Rm327 }\end{array}$

3270418 rpp $378.75 \quad 396.75 \quad 680 \quad 683 \quad 442883.9$ \$E Block wl

3270518 rpp $396.75 \quad 399.75 \quad 678.5 \quad 813.5 \quad 442883.9$ \$E Block wl

3270618 rpp $399.75 \quad 422.25 \quad 810.5 \quad 813.5 \quad 442883.9$ \$E Block wl 


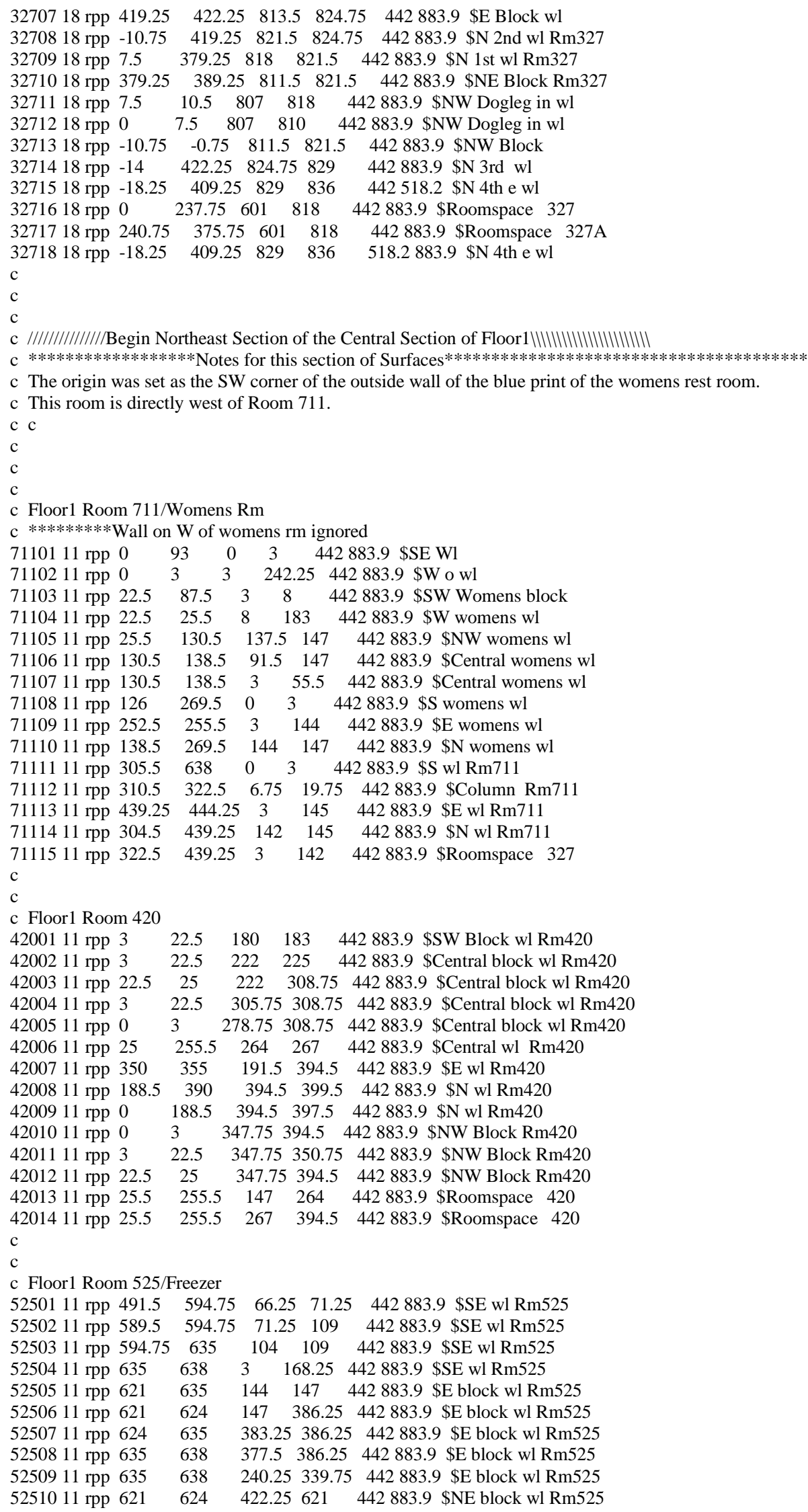




\begin{tabular}{|c|c|c|c|}
\hline 5251111 грp 624 & 638 & 460 & 442883.9 \$NE block wl Rm525 \\
\hline 5251211 rрp 635 & 638 & 463 & 442883.9 \$NE block wl Rm525 \\
\hline 5251311 грp 624 & 635 & 621 & 442883.9 \$NE block wl Rm525 \\
\hline 5251411 rpp 191.5 & 621 & $525 \quad 528$ & 442883.9 \$N wl Rm525 \\
\hline 5251511 rрp 478.5 & 483.5 & 174.5525 & 442883.9 \$Central wl Rm525 \\
\hline 5251611 rрp 483.5 & 514.5 & 174.5179 .5 & 442883.9 \$Central wl Rm525 \\
\hline 5251711 rрp 441.75 & 478.5 & 199204 & 442883.9 \$Central wl Rm525 \\
\hline 5251811 rрp 329 & 334 & $485 \quad 525$ & 442883.9 \$E Freezer wl \\
\hline 5251911 rpp 188.5 & 191.5 & 399.5843 & 442883.9 \$W Freezer wl \\
\hline 5252011 rpp 355 & 478.5 & $204 \quad 394.5$ & 442883.9 \$Roomspace 525 \\
\hline 5252111 rрp 334 & 478.5 & 399.5525 & 442883.9 \$Roomspace 525 \\
\hline 5252211 rрp 483.5 & 621 & $179.5 \quad 525$ & 442883.9 \$Roomspace 525 \\
\hline \\
\hline \\
\hline \multicolumn{4}{|c|}{ c Floor1 Room 426/426A } \\
\hline 4260111 rpp 3 & 83 & $493.5498 .5 \quad 4$ & 442883.9 \$S wl Rm426 \\
\hline 4260211 rpp 125 & 188.5 & 493.5498 .5 & 442883.9 \$S wl Rm426 \\
\hline 4260311 rpp 156.75 & 188.5 & 700.5703 .5 & 442883.9 \$N wl Rm426 \\
\hline 4260411 rpp 79.5 & 107.75 & 700.5703 .5 & 442883.9 \$N wl Rm426 \\
\hline 4260511 rpp 69.5 & 79.5 & 636.25703 .5 & 442883.9 \$E Closet wl Rm426 \\
\hline 4260611 rpp 76.5 & 79.5 & 578.25636 .25 & 442883.9 \$E Closet wl Rm426 \\
\hline 4260711 грp 69 & 76.5 & 578.25581 .25 & 442 883.9 \$SE Closet wl Rm426 \\
\hline 4260811 rpp 4 & 33 & 578.25581 .25 & 442883.9 \$SW Closet wl Rm426 \\
\hline 4260911 rpp 4 & 25 & 581.25591 .75 & 442883.9 \$SW Closet Block wl Rm426 \\
\hline 4261011 rpp 4 & 29 & 591.75596 .75 & 442883.9 \$SW Closet Block wl Rm426 \\
\hline 4261111 rpp 0 & 3 & $548.75578 .25 \quad 4$ & 442883.9 \$SW Closet wl Rm426 \\
\hline 4261211 rрp -24 & -1 & 572.75575 .75 & 442883.9 \$SW Closet wl Rm426 \\
\hline 4261311 rрp -1 & 0 & 575.75578 .25 & 442883.9 \$Very Small Wall Remainder SW wl Rm426 \\
\hline 4261411 rpp -1 & 4 & 578.25645 .5 & 442883.9 \$W Closet wl Rm426 \\
\hline 4261511 rрp 4 & 30 & 639.25645 .5 & 442883.9 \$NW Closet wl Rm426 \\
\hline 4261611 rpp 25 & 30 & 645.5697 .25 & 442883.9 \$NW Closet wl Rm426 \\
\hline 4261711 rpp 25 & 69.5 & 697.25703 .5 & 442883.9 \$N Closet wl Rm426 \\
\hline 4261811 rpp -1 & 25 & 692.75703 .5 & 442883.9 \$N Closet wl Rm426 \\
\hline 4261911 rpp 0 & 3 & 397.5513 .75 & 442883.9 \$W wl Rm426A \\
\hline 4262011 rpp 3 & 188.5 & 397.5493 .5 & 442883.9 \$Roomspace 426A \\
\hline 4262111 rpp 79.5 & 188.5 & 498.5700 .5 & 442883.9 \$Roomspace 426 \\
\hline \multicolumn{4}{|r|}{ 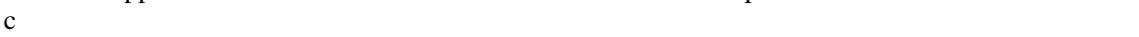 } \\
\hline \multicolumn{4}{|l|}{ C } \\
\hline \multicolumn{4}{|c|}{ c Floor1 Room 430/Receiving Room } \\
\hline 4300111 грp -1 & 27 & 703.5737 .75 & 442883.9 \$W wl Rm430 \\
\hline 4300211 rрp -1 & 2 & 309.75843 & 442883.9 \$W wl Rm430 \\
\hline 4300311 rpp 2 & 22.5 & 827.75840 & 442883.9 \$NW block Rm430 \\
\hline 4300411 rрp 2 & 76.5 & 840843 & 142883.9 \$N wl Rm430 \\
\hline 4300511 rpp -23.5 & 76.5 & 843851 & 442518.2 \$N o wl Rm430 \\
\hline 4300611 rpp -23.5 & -1 & 837.75843 & 442883.9 \$NE wl Rm430 \\
\hline 4300711 rpp 177.5 & 208.5 & 843851 & 442883.9 \$N Cap wl Rm430 \\
\hline 4300811 rpp -9 & $37 \quad 9$ & $928 \quad 930 \quad 44$ & 42 883.9 \$NW Receiving Room \\
\hline 4300911 rpp -9 & -7 & $930 \quad 1005.25 \quad 4$ & 442883.9 \$NW Receiving Room \\
\hline 4301011 rрp $-7 \quad 1$ & 157.4 & 1003.251005 .2 & 25442883.9 \$NW Receiving Room \\
\hline 4301111 rpp 155.4 & 157.4 & $928 \quad 1003.2$ & 25442883.9 \$NW Receiving Room \\
\hline 4301211 грp 111 & 155.4 & 928930 & 442883.9 \$NW Receiving Room \\
\hline 4301311 rpp 177.5 & 188.5 & 840843 & 442883.9 \$NE wl Rm430 \\
\hline 4301411 rpp 2 & 188.5 & 703.5840 & 442883.9 \$Roomspace 430 \\
\hline 4301511 rpp -23.5 & 76.5 & 843851 & 518.2883 .9 \$N o wl Rm430 \\
\hline \multicolumn{4}{|l|}{ C } \\
\hline \multicolumn{4}{|c|}{ c } \\
\hline \multicolumn{4}{|c|}{ c Floor1 Room 527/527A } \\
\hline 5270111 rpp 199.5 & 621 & 536539 & 442883.9 \$S wl Rm527A \\
\hline 5270211 rpp 489 & 621 & 616.75619 .75 & 442883.9 \$N wl Rm527A \\
\hline 5270311 rрp 402 & 453 & 616.75619 .75 & 442883.9 \$NW wl Rm527A \\
\hline 5270411 rрp 399 & 402 & 539619.75 & 442883.9 \$W wl Rm527A \\
\hline 5270511 rpp 322.5 & 325.5 & 539619.75 & 442883.9 \$SW wl in SW Room of Rm527 \\
\hline 5270611 грp 309.5 & 322.5 & 579.75582 .75 & 75442883.9 \$Box in SW wl Rm527 \\
\hline 5270711 rрp 309.5 & 312.5 & 582.75595 .75 & 442883.9 \$Box in SW wl Rm527 \\
\hline 5270811 rрp 312.5 & 322.5 & 592.75595 .75 & 442883.9 \$Box in SW wl Rm527 \\
\hline 5270911 rрp 294.5 & 322.5 & 616.75619 .75 & 442883.9 \$N wl in SW Room of Rm527 \\
\hline 5271011 rpp 202.5 & 237 & 616.75619 .75 & 5442883.9 \$N wl in SW Room of Rm527 \\
\hline 5271111 rpp 199.5 & 202.5 & 539843 & 442883.9 \$W wl Rm527 \\
\hline 5271211 грp 202.5 & 208.5 & 840843 & 442883.9 \$NW small wl Rm527 \\
\hline 5271311 rрp 304.5 & 663 & 843851 & 442518.2 \$N o Major wl Rm527 \\
\hline
\end{tabular}




\begin{tabular}{|c|c|c|c|}
\hline 5271411 грp 304.5 & 310.5 & 840 & 442883.9 \$N i minor wl Rm527 \\
\hline 5271511 грp 307.5 & 310.5 & 830 & 442883.9 \$N i minor wl Rm527 \\
\hline 5271611 rрp 310.5 & 328.5 & 830 & 442883.9 \$N i minor wl Rm527 \\
\hline 5271711 rpp 325.5 & 328.5 & 833 & 442883.9 \$N i minor wl Rm527 \\
\hline 5271811 rpp 325.5 & 372.5 & 840843 & 442883.9 \$N i minor wl Rm527 \\
\hline 5271911 rрp 369.5 & 372.5 & 810.5840 & 442883.9 \$N i minor wl Rm527 \\
\hline 5272011 rpp 372.5 & 383.5 & 813.5816 .5 & 442883.9 \$N i minor wl Rm527 \\
\hline 5272111 rрp 380.5 & 383.5 & 816.5843 & 442883.9 \$N i minor wl Rm527/Rm529 \\
\hline 5272211 rpp 369.5 & 372.5 & 696743.5 & 442883.9 \$NE wl Rm527 \\
\hline 5272311 rpp 372.5 & 635 & 696699 & 442883.9 \$NE wl Rm527 \\
\hline 5272411 rpp 202.5 & 369.5 & 619.75830 & 442883.9 \$Roomspace 527 \\
\hline 5272511 rрp 402 & 621 & $539 \quad 616.75$ & 442883.9 \$Roomspace 527A \\
\hline 5272611 rрp 304.5 & 663 & 851 & 518.2883 .9 \$N o Major wl Rm527 \\
\hline \\
\hline \\
\hline \multicolumn{4}{|l|}{ с Floor1 Room 529} \\
\hline 5290111 rрp 372.5 & 383.5 & 737.5740 .5 & 442883.9 \$W wl Rm529 \\
\hline 5290211 rрp 380.5 & 383.5 & $707 \quad 737.5$ & 442883.9 \$W wl Rm529 \\
\hline 5290311 rpp 383.5 & 621 & 710 & 442883.9 \$S wl Rm529 \\
\hline 5290411 rpp 621 & 624 & 699756 & 442 883.9 \$SE wl Rm529 \\
\hline 5290511 rpp 624 & 635 & $753 \quad 756$ & 442 883.9 \$SE wl Rm529 \\
\hline 5290611 rpp 635 & 638 & 694.5757 .5 & 442883.9 \$SE wl Rm529 \\
\hline 5290711 rpp 635 & 638 & 793.5843 & 442883.9 \$E wl Rm529 \\
\hline 5290811 rpp 638 & 663 & 840843 & 442883.9 \$NE wl Rm529 \\
\hline 5290911 rpp 621 & 635 & 798 & 442883.9 \$NE wl Rm529 \\
\hline 5291011 rpp 621 & 624 & 834 & 442883.9 \$NE wl Rm529 \\
\hline 5291111 rpp 613 & 621 & 834 & 442883.9 \$NE wl Rm529 \\
\hline 5291211 грр 613 & 616 & 834 & 442883.9 \$NE wl Rm529 \\
\hline 5291311 грp 383.5 & 613 & 843 & 442883.9 \$N wl Rm529 \\
\hline 5291411 rрp 341.5 & 530 & 851 & 442883.9 \$S Stairwell wl Rm529 \\
\hline 5291511 грp 341.5 & 630 & 917.5 & 442883.9 \$N stairwell wl Rm529 \\
\hline 5291611 rpp 383.5 & 621 & 710 & 442883.9 \$Roomspace 529 \\
\hline \multicolumn{4}{|r|}{ 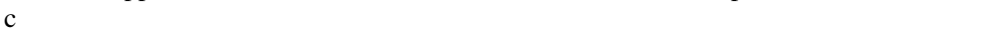 } \\
\hline \multicolumn{4}{|l|}{$\mathrm{C}$} \\
\hline \multicolumn{4}{|l|}{ c Floor1 Room 520} \\
\hline 5200111 грp 719 & 1052 & -6 & 42883.9 \$SW \\
\hline 5200211 rpp 1088 & 1116.5 & $\begin{array}{ll}-6 & -3\end{array}$ & 442883.9 \$SE \\
\hline 5200311 rpp 719 & 722 & $\begin{array}{lll}-3 & 110 \quad 44\end{array}$ & 142883.9 \$SW Box \\
\hline 5200411 rpp 722 & 745 & 105.5108 .5 & 442883.9 \$SW Box \\
\hline 5200511 rpp 742 & 745 & $\begin{array}{lll}-3 & 105.5 & 4\end{array}$ & 442883.9 \$SW Box \\
\hline 5200611 rрp 719 & 722 & $182 \quad 264$ & 442883.9 \$NW Box \\
\hline 5200711 rpp 719 & 722 & 294328 & 442883.9 \$NW Box \\
\hline 5200811 rpp 722 & 745 & 323.5326 .5 & 442883.9 \$NW Box \\
\hline 5200911 rpp 742 & 745 & 183.5323 .5 & 442883.9 \$NW Box \\
\hline 5201011 rpp 722 & 742 & 183.5186 .5 & 442883.9 \$NW Box \\
\hline 5201111 rpp 745 & 1034.5 & 285288 & 442883.9 \$N \\
\hline 5201211 rpp 1070.5 & 1116.5 & 285288 & 442883.9 \$N \\
\hline 5201311 rpp 981.5 & 984.5 & 288538.5 & 442883.9 \$W wl small rm 520-Rm524 \\
\hline 5201411 rpp 984.5 & 1119.5 & 394.25397 .2 & 25442883.9 \$N wl small rm520 \\
\hline 5201511 rpp 1116.5 & 1119.5 & 285394.25 & 5442883.9 \$E wl small rm520 \\
\hline 5201611 rpp 745 & 1116.5 & $-3 \quad 285 \quad 4$ & 442883.9 \$Roomspace 520 \\
\hline 5201711 rpp 984.5 & 1116.5 & $288 \quad 394.25$ & 5442883.9 \$Roomspace $520 \mathrm{~N}$ \\
\hline \multicolumn{4}{|r|}{ 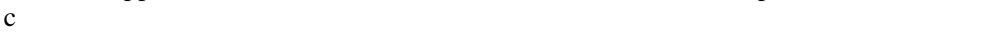 } \\
\hline \multicolumn{4}{|l|}{ c } \\
\hline \multicolumn{4}{|l|}{ c Floor1 Room 524} \\
\hline 5240111 грp 719 & 722 & 397.5632 & 442883.9 \$NW block rm524 \\
\hline 5240211 rpp 722 & 745 & 399402 & 442883.9 \$NW block rm524 \\
\hline 5240311 rрp 742 & 745 & 402630.5 & 442883.9 \$NW block rm524 \\
\hline 5240411 rpp 722 & 742 & 627.5630 .5 & 442883.9 \$NW block rm524 \\
\hline 5240511 rpp 745 & 984.5 & 585588 & 442883.9 \$N wl rm524 \\
\hline 5240611 грp 981.5 & 984.5 & 574.5585 & 442883.9 \$E wl rm524 \\
\hline 5240711 rpp 984.5 & 1005.5 & 497.25500 .2 & 25442883.9 \$E box wl rm524 \\
\hline 5240811 rpp 1002.5 & 1005.5 & 453.25497 .2 & $25 \quad 442883.9$ \$E box wl rm524 \\
\hline 5240911 rpp 984.5 & 1002.5 & 453.25456 .2 & $25 \quad 442883.9$ \$E box wl rm524 \\
\hline 5241011 rрp 745 & 981.5 & 288585 & 442883.9 \$Roomspace 524 \\
\hline \multicolumn{4}{|r|}{ 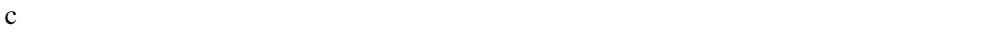 } \\
\hline \multicolumn{4}{|l|}{ 然 } \\
\hline \multicolumn{4}{|l|}{ c Floor1 Room 528} \\
\hline 5280111 rрp 719 & 722 & $704 \quad 771.5$ & 442883.9 \$NE box Rm528 \\
\hline 5280211 rрp 722 & 745 & 705.5708 .5 & 442883.9 \$NE box Rm528 \\
\hline
\end{tabular}




\begin{tabular}{|c|c|c|c|}
\hline 5280311 грp 742 & 745 & 708.5733 & 442883.9 \$NE box Rm528 \\
\hline 5280411 rрp 722 & 737 & $767 \quad 770$ & 442883.9 \$NE box Rm528-Rm530 \\
\hline 5280511 rрp 734 & 737 & 741 & 442883.9 \$NE box Rm528-Rm530 \\
\hline 5280611 rpp 745 & 897.5 & 730 & 442883.9 \$NW wl Rm528 \\
\hline 5280711 rpp 897.5 & 900.5 & $730 \quad 840$ & 442883.9 \$NW wl Rm528 \\
\hline 5280811 rpp 800.75 & 1119.75 & $75 \quad 840 \quad 843$ & 442883.9 \$N i wl Rm528-Rm530 \\
\hline 5280911 rpp 1119.75 & 51129 & -21.5843 & 442883.9 \$W wl Rm528 \\
\hline 5281011 rpp 800.75 & 1137 & 843851 & 442518.2 \$N o wl Rm528-Rm530 \\
\hline 5281111 rpp 897.5 & 1119.5 & $588 \quad 840$ & 442883.9 \$Roomspace 528 Partially Filled \\
\hline 5281211 rpp 800.75 & 1137 & 843 & 518.2883 .9 \$N o wl Rm528-Rm530 \\
\hline \\
\hline \\
\hline \multicolumn{4}{|c|}{ c Floor1 Room 530/North Cylinder Dock } \\
\hline $5300111 \mathrm{rpp} 737$ & 886.5 & 741744 & 442883.9 \$S wl Rm530 \\
\hline 5300211 грp 883.5 & 886.5 & 744840 & 442883.9 \$E wl Rm530 \\
\hline 5300311 rрp 699 & 764.5 & 840843 & 442883.9 \$NW wl Rm530 \\
\hline 5300411 rрp 719 & 722 & 806.5840 & 442883.9 \$NW block wl Rm530 \\
\hline 5300511 грp 722 & 734 & 808811 & 442883.9 \$NW block wl Rm530 \\
\hline 5300611 rpp 734 & 737 & $808 \quad 834$ & 442883.9 \$NW block wl Rm530 \\
\hline 5300711 грp 737 & 745 & 831834 & 442883.9 \$NW block wl Rm530 \\
\hline 5300811 грp 742 & 745 & 834840 & 442883.9 \$NW block wl Rm530 \\
\hline 5300911 грp 711 & 719 & 851941 & 442883.9 \$Cylinder Dock wl \\
\hline 5301011 грp 815 & 823 & 851941 & 442883.9 \$Cylinder Dock wl \\
\hline 5301111 rрp 948 & 956 & 851941 & 442883.9 \$Cylinder Dock wl \\
\hline 5301211 rpp 1062 & 1070 & 851941 & 442883.9 \$Cylinder Dock wl \\
\hline 5301311 rрp 1137 & 1162 & 777.5781 .5 & 442883.9 \$Cylinder Dock small wl \\
\hline 5301411 rрp 1137 & 1162 & 813817 & 442883.9 \$Cylinder Dock small wl \\
\hline 5301511 rpp 1129 & 1137 & 851941 & 442883.9 \$Cylinder Dock wl \\
\hline 5301611 rpp 699 & 764.5 & 843851 & 442518.2 \$Cylinder Dock exterior wl \\
\hline 5301711 грp 737 & 883.5 & 744840 & 442883.9 \$Roomspace 530 \\
\hline 5301811 грр 699 & 764.5 & 843851 & 518.2883 .9 \$Cylinder Dock exterior wl \\
\hline \multicolumn{4}{|r|}{ 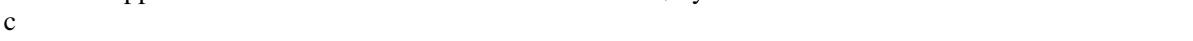 } \\
\hline \multicolumn{4}{|c|}{ c } \\
\hline \multicolumn{4}{|c|}{ c //////I///////Begin West Section of Floor1/IIIIIIIIIIIIIIIIIIIIII } \\
\hline \multicolumn{4}{|c|}{ c $* * * * * * * * * * * * * * * * * *$ Notes for this section of Surfaces $* * * * * * * * * * * * * * * * * * * * * * * * * * * * * * * * * * * * * * * * *$} \\
\hline \multirow{2}{*}{\multicolumn{4}{|c|}{$\begin{array}{l}\text { c The origin was set as the SW corner of the inside wall of Room 209. For conservancy the hot } \\
\text { c cells in SAL were modled as a solid RPP of metal. }\end{array}$}} \\
\hline & & & \\
\hline \multicolumn{4}{|c|}{ C } \\
\hline \multicolumn{4}{|l|}{$\mathrm{C}$} \\
\hline \multicolumn{4}{|l|}{ C } \\
\hline \multicolumn{4}{|l|}{ c Floor1 Room 200} \\
\hline 2000112 грp -174 & -161.25 & 5485.5487 .5 & 442883.9 \$SW thin wl Rm200 \\
\hline 2000212 rpp -89 & -824 & 485.5487 .5 & 442883.9 \$SW thin wl Rm200 \\
\hline 2000312 rpp -85.5 & -83.5 & 410.5485 .5 & 442883.9 \$SW thin wl Rm200 \\
\hline 2000412 rpp -87 & -824 & 406.5410 .5 & 442883.9 \$SW thin wl Rm200 \\
\hline 2000512 rpp -87 & -82 & 402.5406 .5 & 442883.9 \$SW thin wl Rm200 \\
\hline 2000612 грр -46 & -13 & 485.5487 .5 & 442883.9 \$SW thin wl Rm200 \\
\hline 2000712 грp 89 & 91 & 410.5422 & 442883.9 \$S divider thin wl Rm200 \\
\hline 2000812 rрp 89 & 91 & $458 \quad 485.5 \quad 4$ & 442883.9 \$S divider thin wl Rm200 \\
\hline 2000912 грр -13 & -11 & 955.4990 .25 & 442883.9 \$NW thin wl Rm200 \\
\hline 2001012 rрp -13 & -11 & 10381044.5 & 442883.9 \$NW thin wl Rm200 \\
\hline 2001112 rpp -45.25 & 28 & 406.5410 .5 & 442883.9 \$S i wl Rm200 \\
\hline 2001212 rpp -45.25 & 28 & 402.5406 .5 & 442883.9 \$S o wl Rm200 \\
\hline 2001312 rpp 66 & 250.25 & 406.5410 .5 & 442883.9 \$S i wl Rm200-Rm201 \\
\hline 2001412 rpp 66 & 246 & 402.5406 .5 & 442883.9 \$S o wl Rm200-Rm201 \\
\hline C & & & \\
\hline C & & & \\
\hline c Floor1 Room 201 & & & \\
\hline $\mathrm{C} * * * * * * * * * * * * * * * *$ & ${ }^{k}$ This roo & om contains the & e actual SAL hot cells************** \\
\hline c Neglected thin wall & strip in I & NE border regi & ion There are two types of concrete in SAL- \\
\hline c Heavy Aggregate an & nd Sump & concrete. Curr & rently only std concrete is being used for both. \\
\hline c The roof and floor.. & ......... & & \\
\hline 2010112 rpp 250.25 & 266.25 & 5457.5481 & 442883.9 \$E Block Rm201 \\
\hline 2010212 rpp 266.25 & 345 & 464.25469 .2 & 25442883.9 \$E wl Rm201 \\
\hline 2010312 rpp 250.25 & 266.25 & 5613630 & 442883.9 \$E Column Rm201 \\
\hline 2010412 rpp 253.5 & 263.5 & 854.5864 .5 & 442883.9 \$NE column Rm201 \\
\hline 2010512 rpp 263.5 & 307.5 & 857862 & 442883.9 \$NE wall off of column Rm201 \\
\hline 2010612 rpp 265 & 266.5 & 8621037 & 442883.9 \$NE wl Rm201 \\
\hline 2010712 rpp 263.5 & 266.5 & 10371335.5 & 5442883.9 \$NE wl Rm201 \\
\hline 2010812 rpp 253.5 & 263.5 & $1037 \quad 1335.5$ & 5442518.2 \$NE wl Rm201 \\
\hline
\end{tabular}




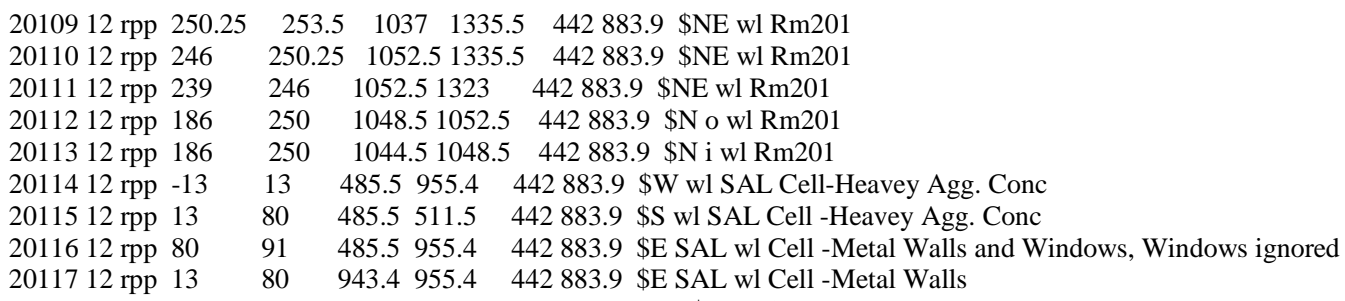


c Floor1 Room 301

3010113 rpp $-237 \quad 3 \quad-3.5 \quad 0 \quad 442883.9$ \$S wl Rm301

3010213 rpp $0 \quad 3 \quad 0 \quad 42 \quad 442883.9$ \$SE block wl Rm301

3010313 rpp $3 \quad 21 \quad 39 \quad 42 \quad 442883.9$ \$SE block wl Rm301

3010413 rpp $21 \quad 24 \quad 0 \quad 43.75 \quad 442883.9$ \$SE block wl Rm301

3010513 rpp $3 \quad 24 \quad-3 \quad 0 \quad 442883.9$ \$SE block wl Rm301

3010613 rpp $0 \quad 3 \quad 81.5 \quad 156.75 \quad 442883.9$ \$NE block wl Rm301

$\begin{array}{llllll}30107 & 13 \text { rpp } 3 & 21 & 153.75 & 156.75 & 442883.9 \\ \text { \$NE block wl Rm301 }\end{array}$

3010813 rpp $21 \quad 24 \quad 79.75 \quad 157.25 \quad 442$ 883.9 $\quad$ \$NE block wl Rm301

3010913 rpp $3 \quad 21 \quad 81.5 \quad 84.5 \quad 442883.9$ \$NE block wl Rm301

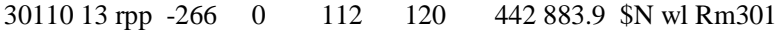

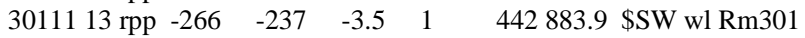

3011213 rpp $-271 \quad-266 \quad-3.5 \quad 356 \quad 442883.9$ \$W wl Rm301-Rm305

3011313 rpp $-266 \quad 0 \quad 0 \quad 112 \quad 442883.9$ \$Roomspace 301

c Floor1 Room 303

3030113 rpp $-266 \quad 0 \quad 120 \quad 127.5 \quad 442883.9$ \$S i wl Rm303

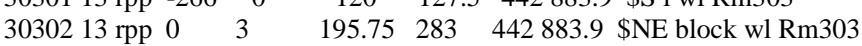

3030313 rpp $3 \quad 21 \quad 280 \quad 283 \quad 442883.9$ \$NE block wl Rm303

3030413 rpp $21 \quad 24 \quad 255.25 \quad 284.25442883 .9$ \$NE block wl Rm303

3030513 rpp $21 \quad 24 \quad 195.25224 .75442$ 883.9 \$NE block wl Rm303

3030613 rpp $3 \quad 21 \quad 195.75198 .75442883 .9$ \$NE block wl Rm303

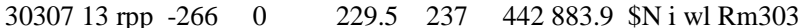

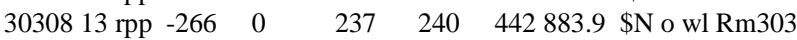

3030913 rpp $-266 \quad 0 \quad 127.5 \quad 229.5442883 .9$ \$Roomspace 303

C

c Floor1 Room 305

3050113 rpp $-249.5 \quad 0 \quad 240 \quad 247.5 \quad 442883.9$ \$S wl Rm305

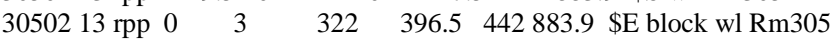

3050313 rpp $3 \quad 21 \quad 393.5 \quad 396.5442883 .9$ \$E block wl Rm305

3050413 rpp $21 \quad 24 \quad 320.75398 \quad 442883.9$ \$E block wl Rm305

\begin{tabular}{lllll}
30505 & 13 rpp 3 & 21 & 325 & 328 \\
\hline
\end{tabular}

3050613 rpp $0 \quad 3 \quad 435.5 \quad 522.25442883 .9$ \$NE block wl Rm305

3050713 rpp $3 \quad 21 \quad 519.25 \quad 522.25442883 .9$ \$NE block wl Rm305

3050813 rpp $21 \quad 24 \quad 434.25 \quad 522.75442883 .9$ \$NE block wl Rm305

3050913 rpp $3 \quad 21 \quad 435.5 \quad 438.5 \quad 442$ 883.9 \$NE block wl Rm305

3051013 rpp $-292 \quad 0 \quad 469.5 \quad 477 \quad 442883.9$ \$N wl Rm305

3051113 rpp $-292 \quad 0 \quad 477 \quad 480 \quad 442883.9$ \$N wl Rm305

3051213 rpp $-266 \quad 0 \quad 247.5469 .5442883 .9$ \$Roomspace 305 Partially Filled

C

c Floor1 Room 309

3090113 rpp $-292 \quad 0$

3090213 rpp $0 \quad 3$

3090313 rpp $3 \quad 21$

3090413 rpp $21 \quad 24$

3090513 rpp 321

3090613 rpp $-292 \quad 0$

3090713 rpp -292

3090813 rpp $-292 \quad-271$

3090913 rpp $0 \quad 3$

3091013 rpp $3 \quad 21$

3091113 rpp $21 \quad 24$

3091213 rpp 321

3091313 rpp $-292 \quad 0$

C

c Floor1 Room 313

3130113 rpp $-292 \quad 0$

3130213 rpp -292 0

3130313 rpp $-292 \quad 0$

3130413 rpp $0 \quad 3$

3130513 rpp $3 \quad 21$

3130613 rpp $21 \quad 24$

3130713 rpp $3 \quad 21$

3130813 rpp 0

3130913 rpp 3

$480 \quad 487.5 \quad 442883.9$ \$S wl Rm309

$561.25 \quad 636.25442$ 883.9 \$E block wl Rm309

633.25 636.25 442883.9 \$E block wl Rm309

561.75636 .75442883 .9 \$E block wl Rm309

561.25564 .25442883 .9 \$E block wl Rm309

$709.5 \quad 717 \quad 442883.9$ \$N wl Rm309

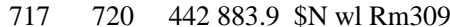

$351 \quad 356 \quad 442883.9$ \$W dogleg in wl Rm305

675.25762 .25442 883.9 \$NE block wl Rm309

759.25762 .25442883 .9 \$NE block wl Rm309

674.75762 .75442883 .9 \$NE block wl Rm309

675.25678 .25442883 .9 \$NE block wl Rm309

487.5709 .5442883 .9 \$Roomspace 309

$949.5957 \quad 442883.9$ \$N wl Rm313

$957 \quad 960 \quad 442883.9$ \$N wl Rm313

$720 \quad 727.5 \quad 442883.9$ \$S wl Rm313

$801.25876 \quad 442883.9$ \$E block wl Rm313

$873 \quad 876 \quad 442883.9$ \$E block wl Rm313

800.75876 .75442883 .9 \$E block wl Rm313

801.25 804.25 442 883.9 \$E block wl Rm313

915996.75442 883.9 \$NE block wl Rm313

993.75996 .75442 883.9 \$NE block wl Rm313 


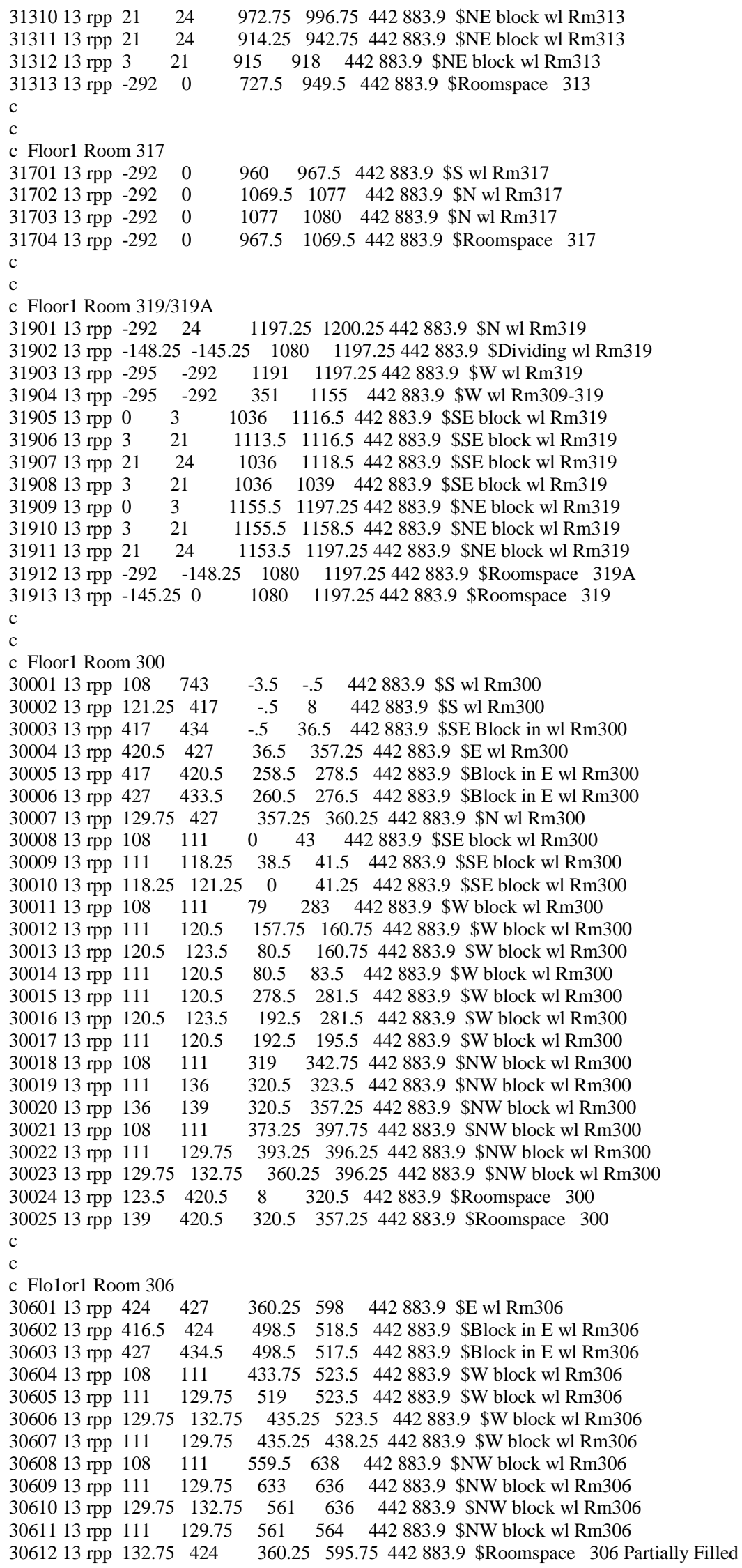




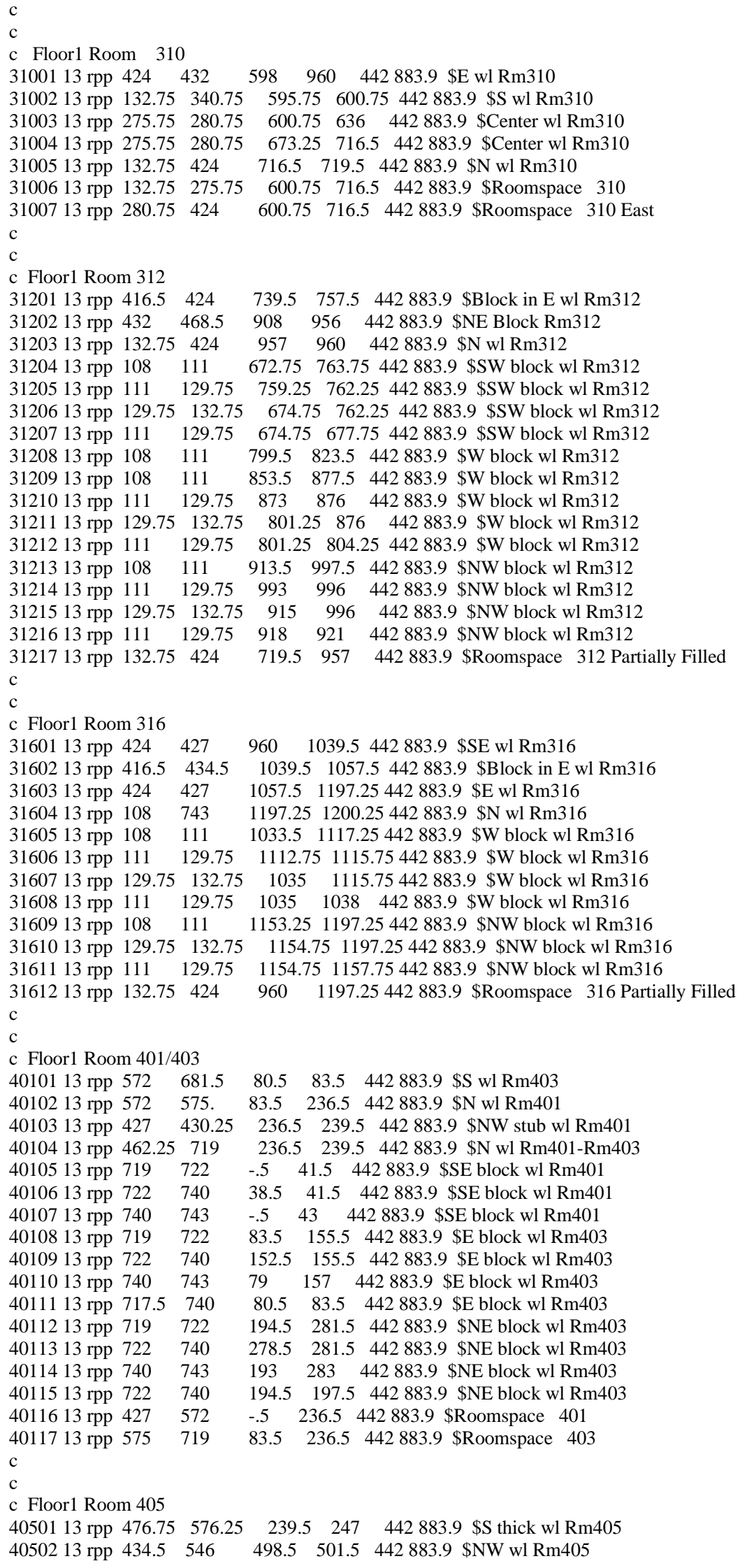




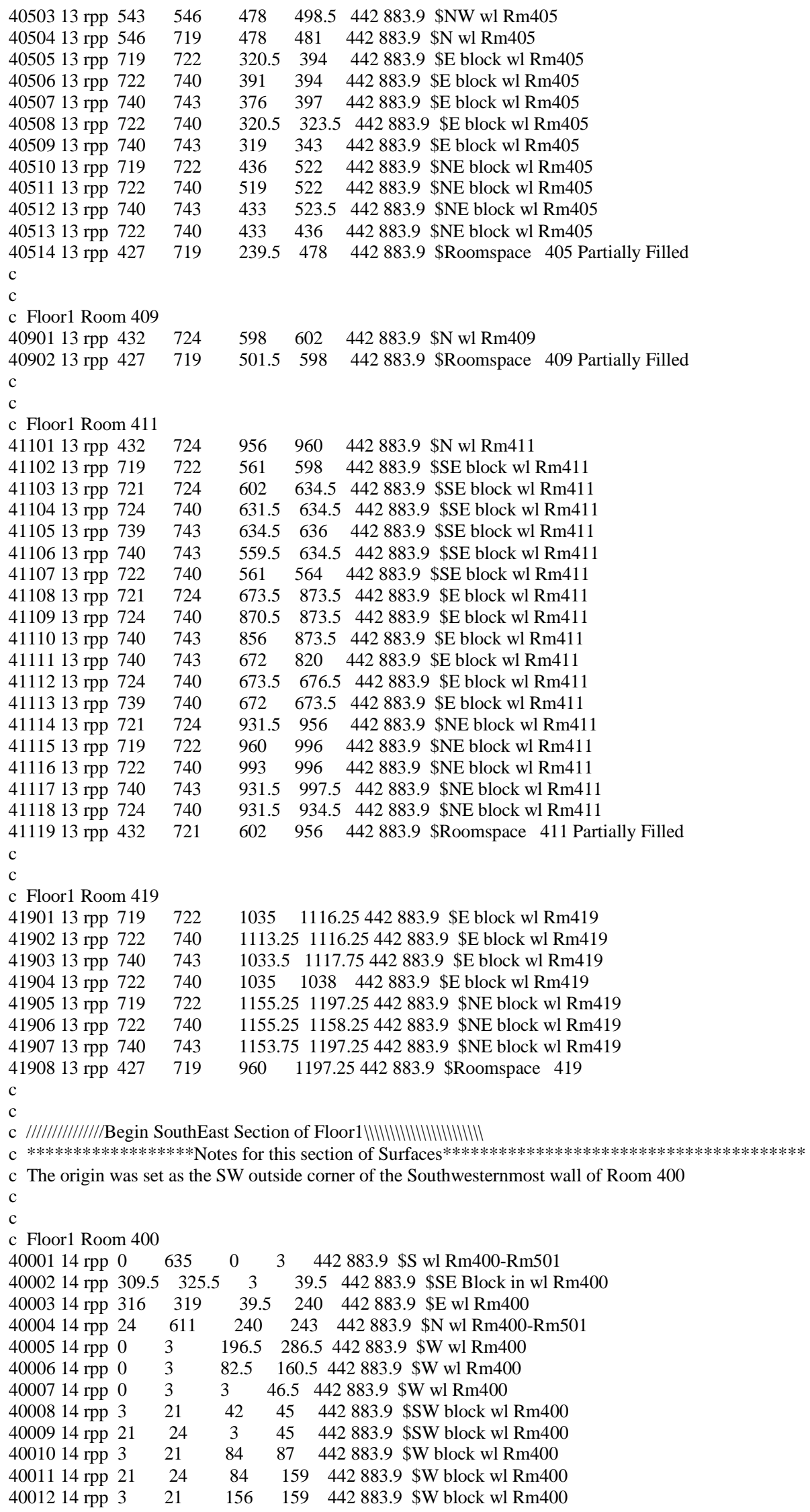




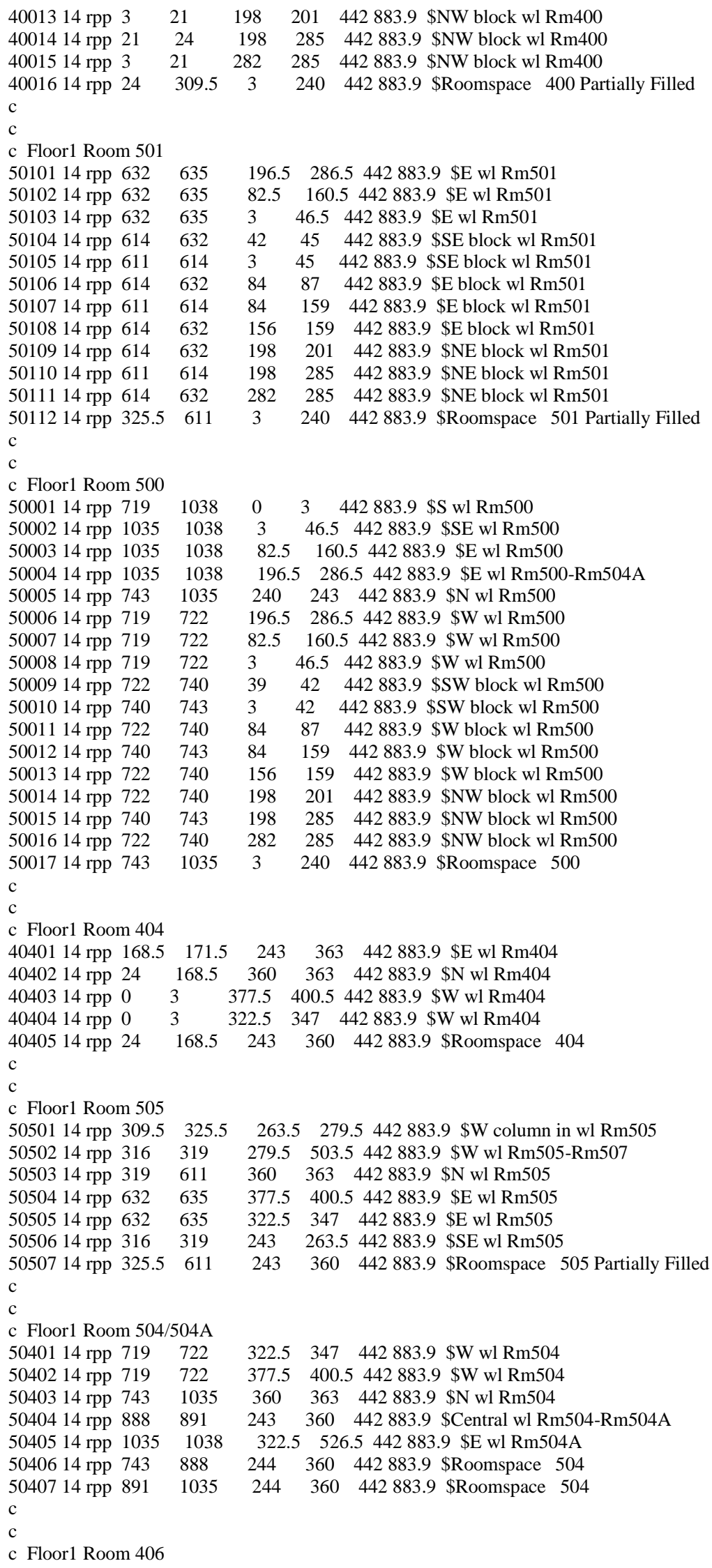




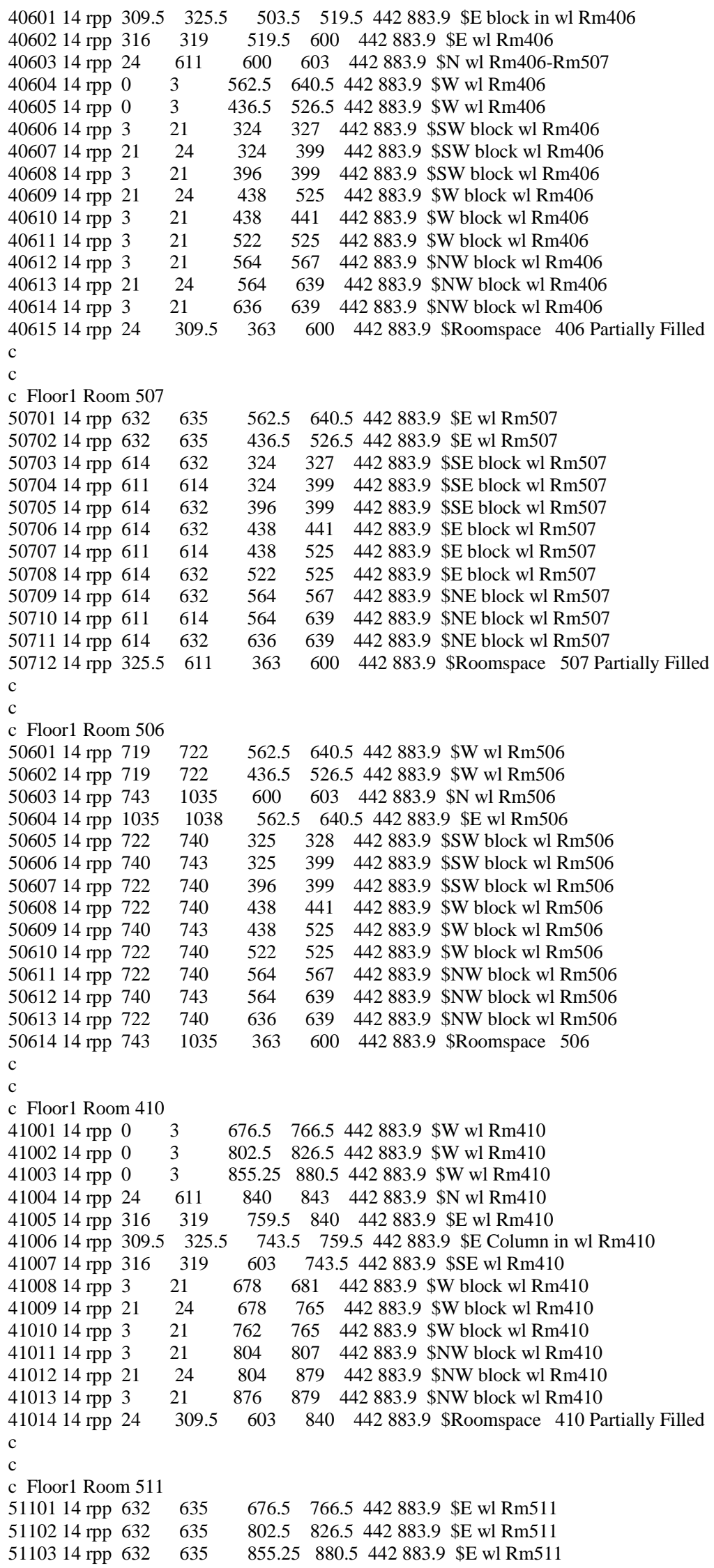

635

635

635

676.5766 .5442883 .9 \$E wl Rm511

802.5826 .5442883 .9 \$E wl Rm511

$855.25 \quad 880.5442883 .9$ \$E wl Rm511 


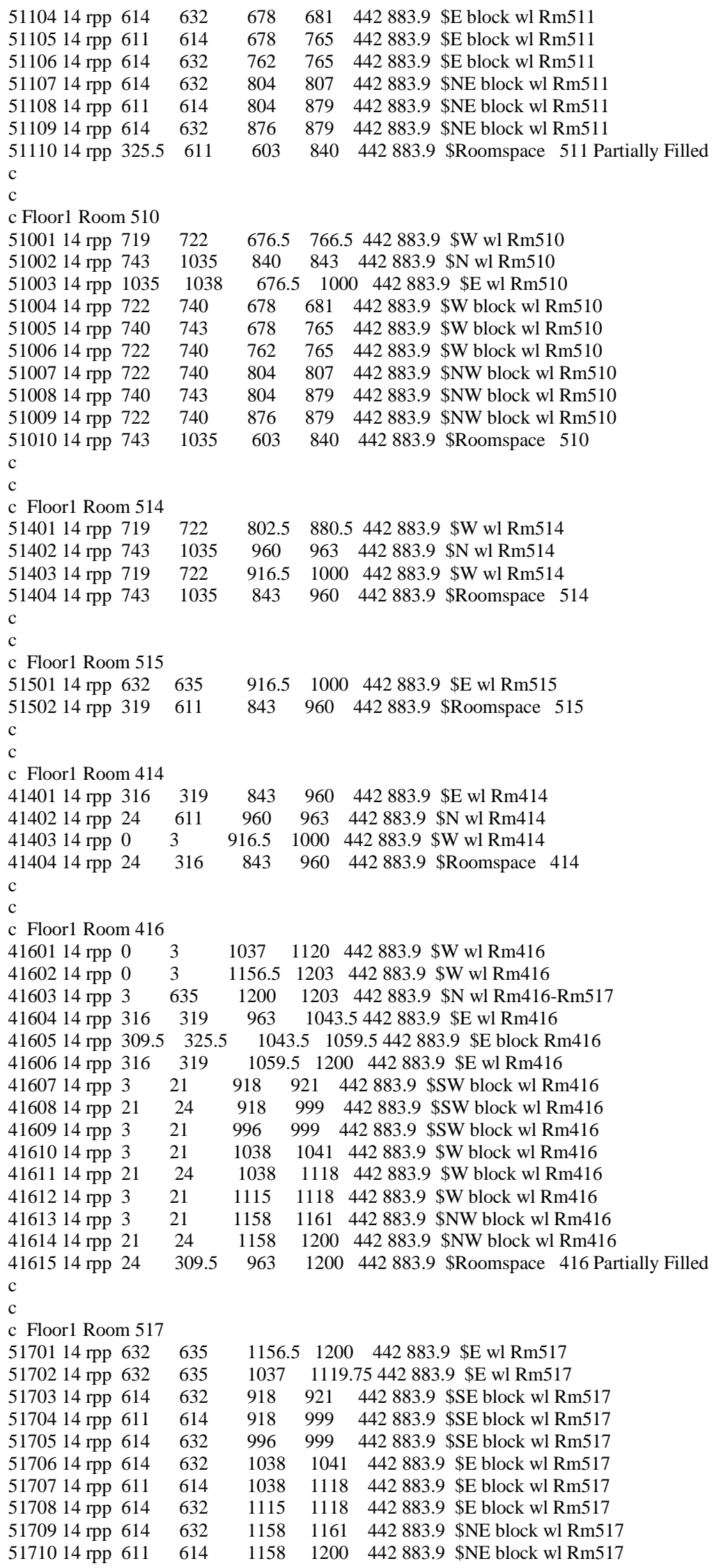




\begin{tabular}{|c|c|c|}
\hline \multicolumn{3}{|r|}{442883.9 \$Roomspace 517 Partially Filled } \\
\hline \multicolumn{3}{|l|}{ C } \\
\hline \multicolumn{3}{|l|}{ c Floor1 Room 516} \\
\hline 5160114 грp 719 & 722 & 1156.51200442883 .9 \$W wl Rm516 \\
\hline 5160214 грp 719 & 722 & $1037 \quad 1119.75442883 .9$ \$W wl Rm516 \\
\hline 5160314 грp 719 & 1038 & 12001203442883.9 \$N wl Rm516 \\
\hline 5160414 rpp 1035 & 1038 & 1156.51200442883 .9 \$E wl Rm516 \\
\hline 5160514 rpp 1035 & 1038 & $1037 \quad 1119.75442883 .9$ \$E wl Rm516 \\
\hline 5160614 rpp 722 & 740 & $918921 \quad 442883.9$ \$SW block wl Rm516 \\
\hline 5160714 грp 740 & 743 & 999442883.9 \$SW block wl Rm516 \\
\hline 5160814 rрp 722 & 740 & 999442883.9 \$SW block wl Rm516 \\
\hline 5160914 грp 722 & 740 & 1041442883.9 \$W block wl Rm516 \\
\hline 5161014 rрp 740 & 743 & 1118442883.9 \$W block wl Rm516 \\
\hline 5161114 rpp 722 & 740 & 1118442883.9 \$W block wl Rm516 \\
\hline 5161214 rрp 722 & 740 & 1161442883.9 \$NW block wl Rm516 \\
\hline 5161314 rрp 740 & 743 & 1200442883.9 \$NW block wl Rm516 \\
\hline 5161414 rpp 743 & 1035 & 1200442883.9 \$Roomspace 516 \\
\hline \multicolumn{3}{|r|}{ 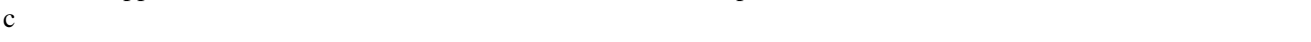 } \\
\hline \multicolumn{3}{|c|}{ C } \\
\hline \multicolumn{3}{|c|}{ c //////////////Begin East Section of Floor1/IIIIIIIIIIIIIIIIIIIIII } \\
\hline \multicolumn{3}{|c|}{ c $* * * * * * * * * * * * * * * * * *$ Notes for this section of Surfaces $* * * * * * * * * * * * * * * * * * * * * * * * * * * * * * * * * * * * * * *$} \\
\hline \multicolumn{3}{|c|}{ c The origin was set as the inside corner of the Southwesternmost wall of the east Section. } \\
\hline \multicolumn{3}{|c|}{ c There is no specific number for this area but the origin is directly west of Room 600 . } \\
\hline \multicolumn{3}{|c|}{ C } \\
\hline \multicolumn{3}{|l|}{ C } \\
\hline \multicolumn{3}{|l|}{ c Floor1 Room 600} \\
\hline 6000115 rрp -16.5 & -13.5 & 178.5442883 .9 \$W most wall, lies on boundary line \\
\hline 6000215 rpp -13.5 & -4 & 178.5442883 .9 \$W wl Rm600 \\
\hline 6000315 грр -4 & $0 \quad 0$ & $0 \quad 178.5442883 .9$ \$W wl Rm600 \\
\hline 6000415 грp -16.5 & -13.5 & 214.51464 .5442883 .9 \$W most wl Rm600-601 \\
\hline 6000515 грp -13.5 & -4 & 214.51461 .5442883 .9 \$W wl Rm600-601 \\
\hline 6000615 грp -4 & $4 \quad 21$ & 214.5994 .5442883 .9 \$W wl Rm600-601 \\
\hline 6000715 грp -16.5 & 580.5 & 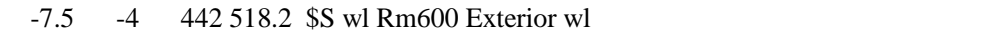 \\
\hline 6000815 rpp -16.5 & 577 & $0 \quad 442883.9$ \$S wl Rm600 \\
\hline 6000915 грp 74 & 79 & 115442883.9 \$W i wl Rm600 \\
\hline 6001015 rpp 74 & 79 & 162.5442883 .9 \$NW wl stub Rm600 \\
\hline 6001115 грp 79 & 205 & 162.5442883 .9 \$N wl Rm600 \\
\hline 6001215 rрp 196 & 201 & 157.5442883 .9 \$E wl Rm600 \\
\hline 6001315 rpp 79 & 196 & $0 \quad 3.5 \quad 442883.9$ \$S wl Rm600 \\
\hline 6001415 rpp 96.5 & 100.5 & $162.5 \quad 187 \quad 442883.9$ \$N wl extension Rm600 \\
\hline 6001515 rpp 4 & 100.5 & $224 \quad 228 \quad 442883.9$ \$NW wl Rm600 \\
\hline 6001615 rpp 79 & 196 & 157.5442883 .9 \$Roomspace 600 \\
\hline 6001715 rрp -16.5 & 580.5 & -4 $\quad 518.2883 .9$ \$S wl Rm600 Exterior wl \\
\hline \multicolumn{3}{|r|}{ ( } \\
\hline C & & \\
\hline c Floor1 Room 602/ & /605/606 & \\
\hline c These rooms were & e all comb & bined because it is difficult to distinguish between them. \\
\hline C & & \\
\hline 6020115 rрp 205 & 209 & 52.25442883 .9 \$SW Column Rm602 \\
\hline 6020215 rpp 241 & 245 & 52.25442883 .9 \$SW 2nd column Rm602 \\
\hline 6020315 rрp 284 & 287 & 204.25442883 .9 \$W wl Womens Rm/605 \\
\hline 6020415 rpp 287 & 291.5 & 53.25442883 .9 \$SW 3rd Column Rm602 \\
\hline 6020515 rpp 321.5 & 413.5 & 53.25442883 .9 \$S wl Rm605 <----------columns within wall were omitted \\
\hline 6020615 rpp 325 & 330 & $53.25 \quad 64.75442883 .9$ \$SW wl Rm605 \\
\hline 6020715 rpp 287 & 330 & 102.75442883 .9 \$SW wl Rm605 \\
\hline 6020815 rрp 325 & 330 & 94.7597 .75442883 .9 \$SW small wl Rm605 \\
\hline 6020915 грp 400.75 & 5405.75 & $5 \quad 46.75 \quad 48.25 \quad 442883.9$ \$SE wall protrusion Rm605 \\
\hline 6021015 грp 400.75 & 5405.75 & $53.25 \quad 166.25442883 .9$ \$E wl Rm605 \\
\hline 6021115 грp 405.75 & 5440.75 & 157.25162 .25442883 .9 \$E wl Rm605 \\
\hline 6021215 rpp 474.75 & $5 \quad 486.75$ & $157.25 \quad 162.25442883 .9$ \$E wl Rm605 \\
\hline 6021315 rрp 481.75 & 5486.75 & $162.25 \quad 199.25442883 .9$ \$E wl Rm605 \\
\hline 6021415 грp 478.5 & 486.75 & $204.25212 \quad 442883.9$ \$NE wl Rm605 \\
\hline 6021515 грp 449.75 & 5486.75 & $199.25 \quad 204.25442883 .9$ \$NE wl Rm605 \\
\hline 6021615 rpp 287 & 405.75 & $199.25 \quad 204.25442883 .9$ \$N wl Rm605 \\
\hline 6021715 грp 400.75 & 5405.75 & $5 \quad 196.25 \quad 199.25442883 .9$ \$N wl stub Rm605 \\
\hline 6021815 rрp 287 & 358 & $195.25 \quad 199.25442883 .9$ \$NW wl fat part Rm605 \\
\hline 6021915 rрp 335 & 338 & $204.25214 \quad 442883.9$ \$Protrusion from N wl in Rm605 into Rm601 \\
\hline 6022015 rрp 486.75 & 5560 & 169442883.9 \$S wl Rm606 \\
\hline 6022115 rpp 560 & 573 & 252442883.9 \$E wl Rm606-Rm202 \\
\hline
\end{tabular}




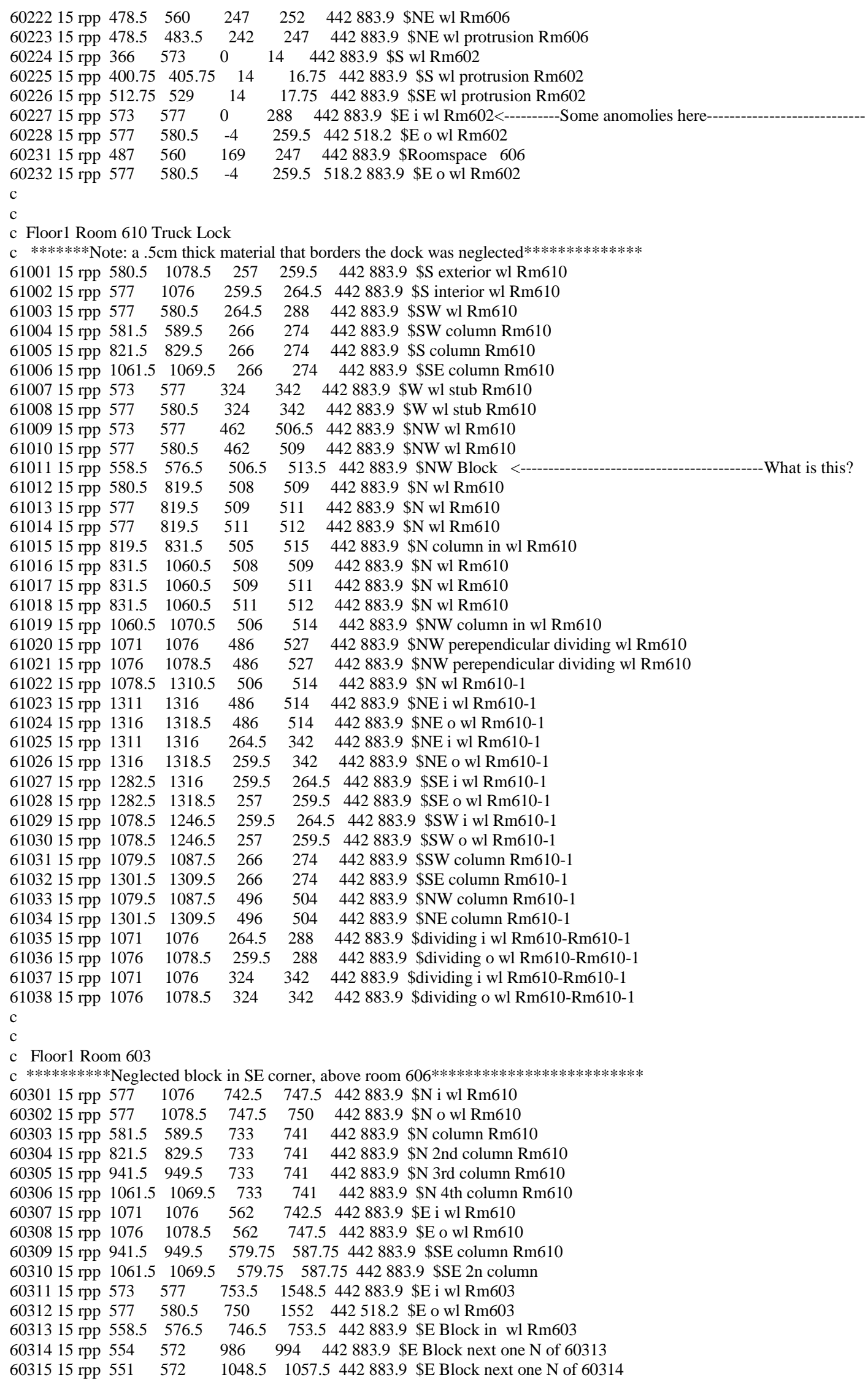




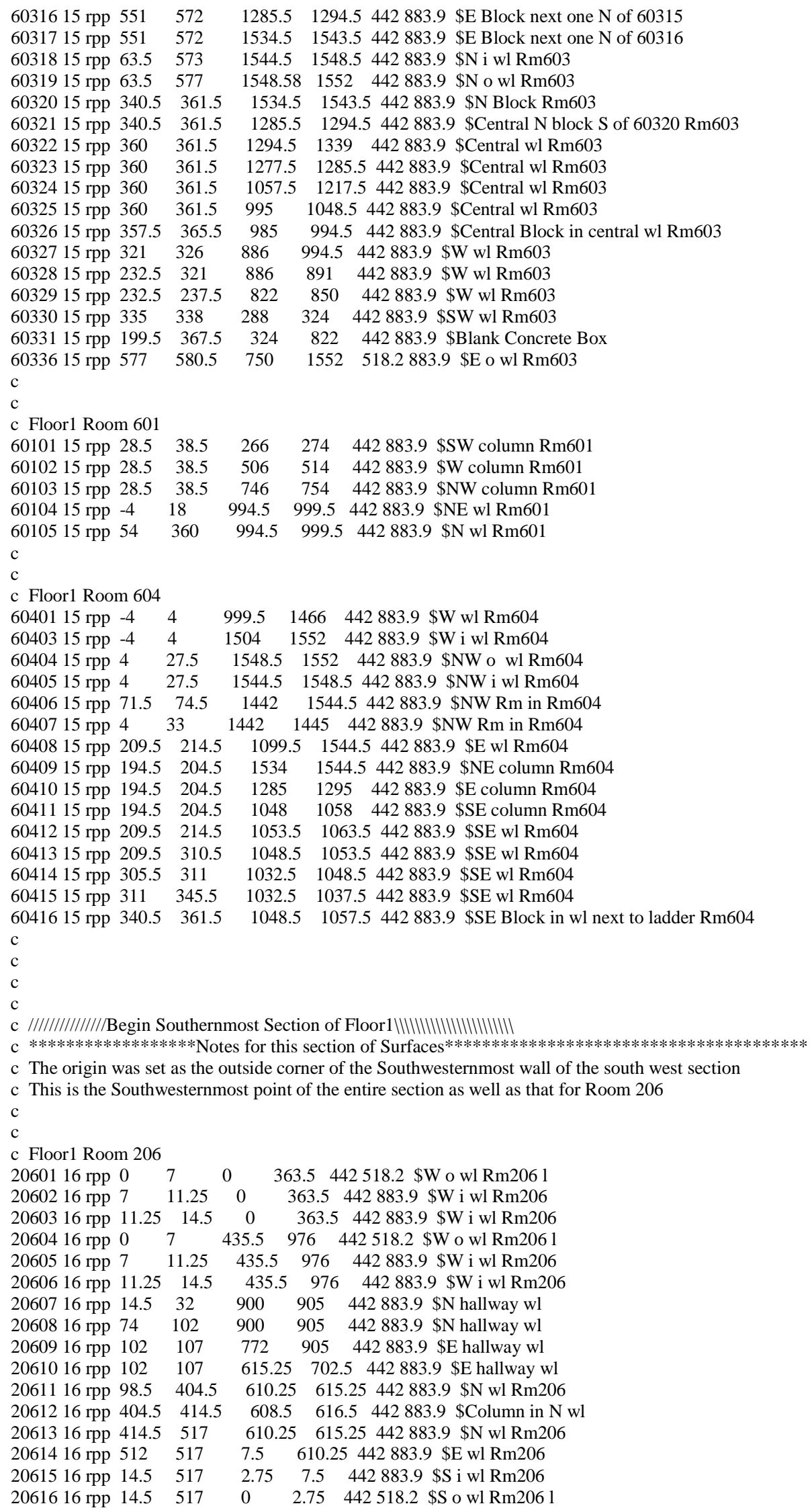




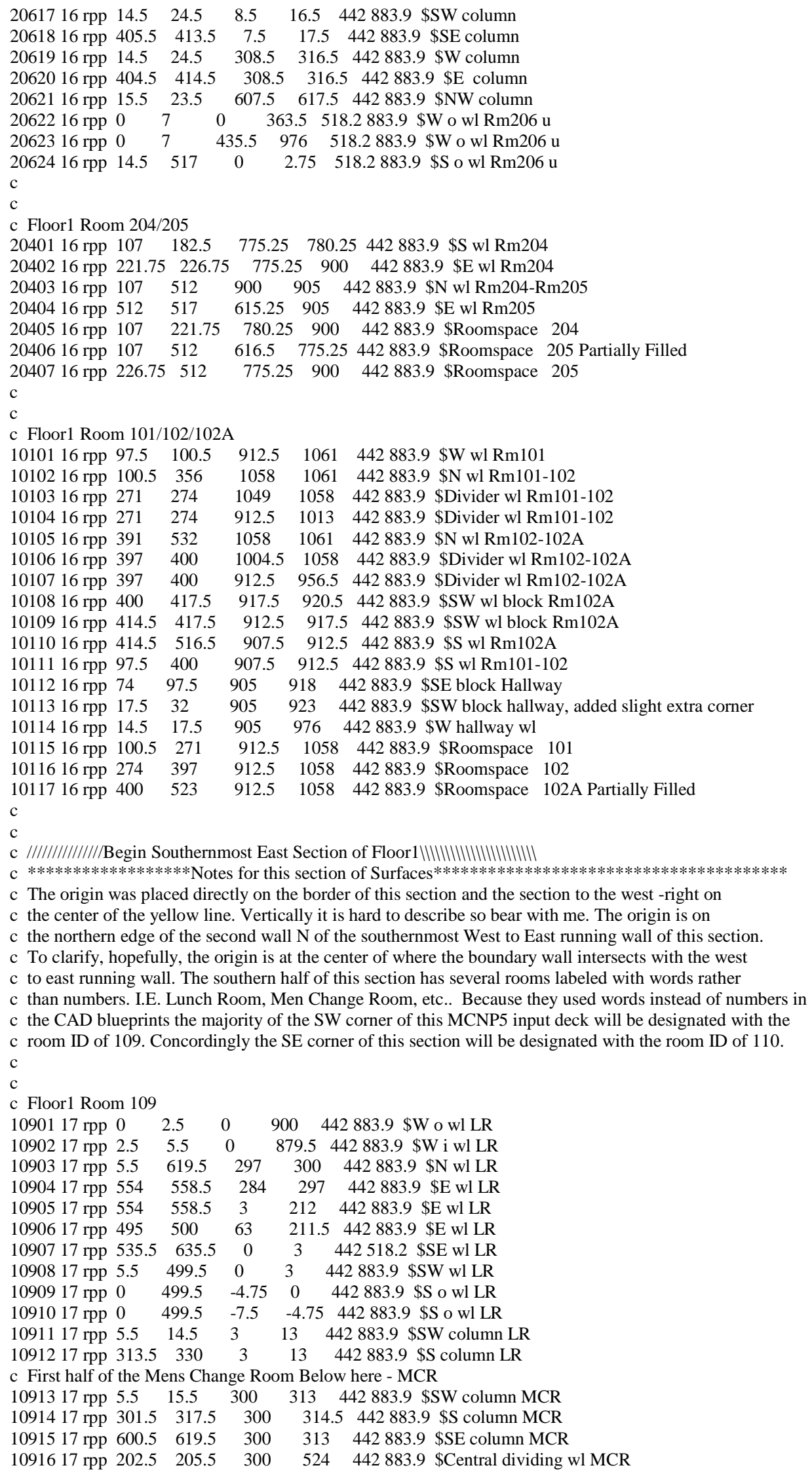




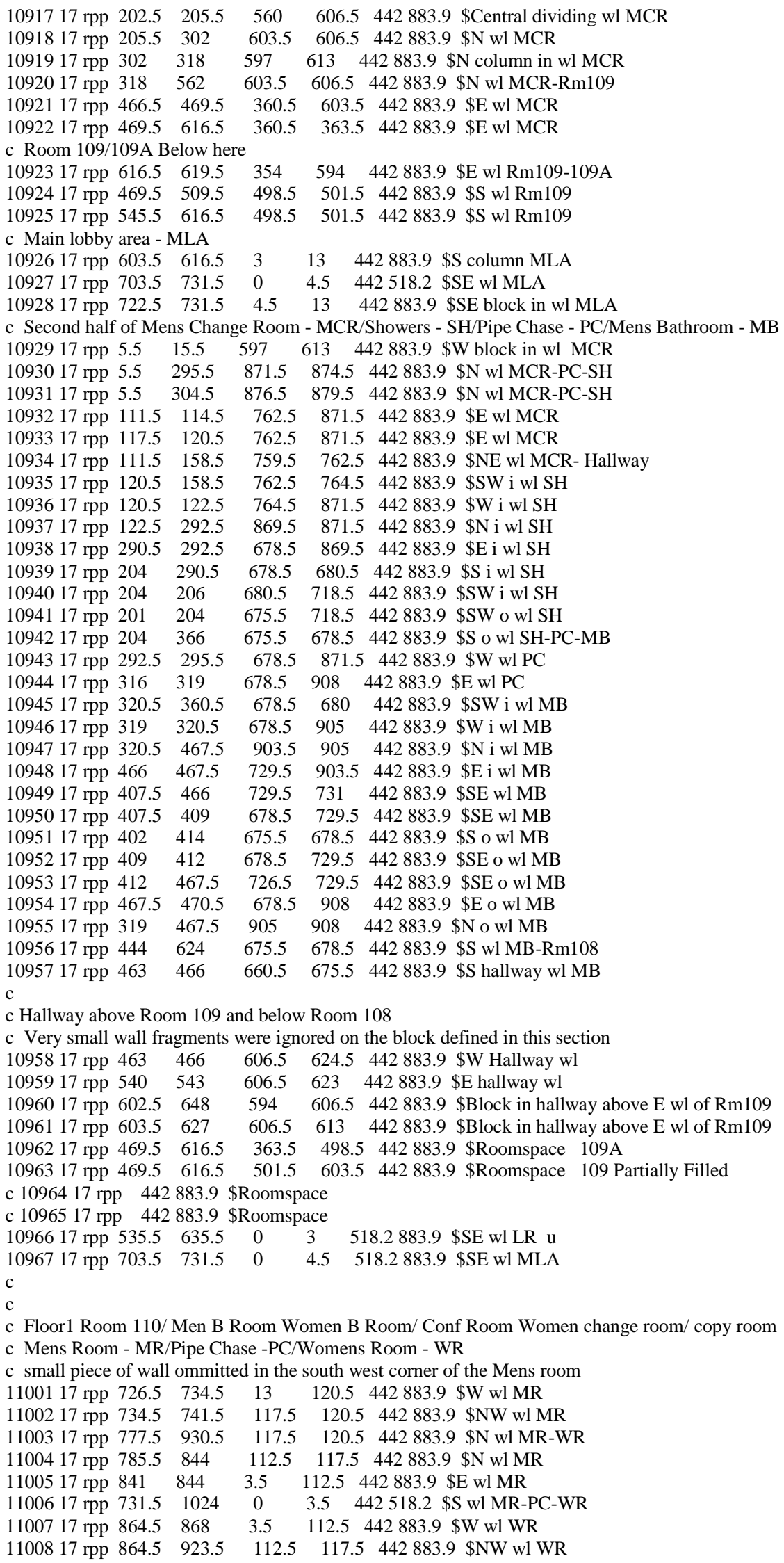




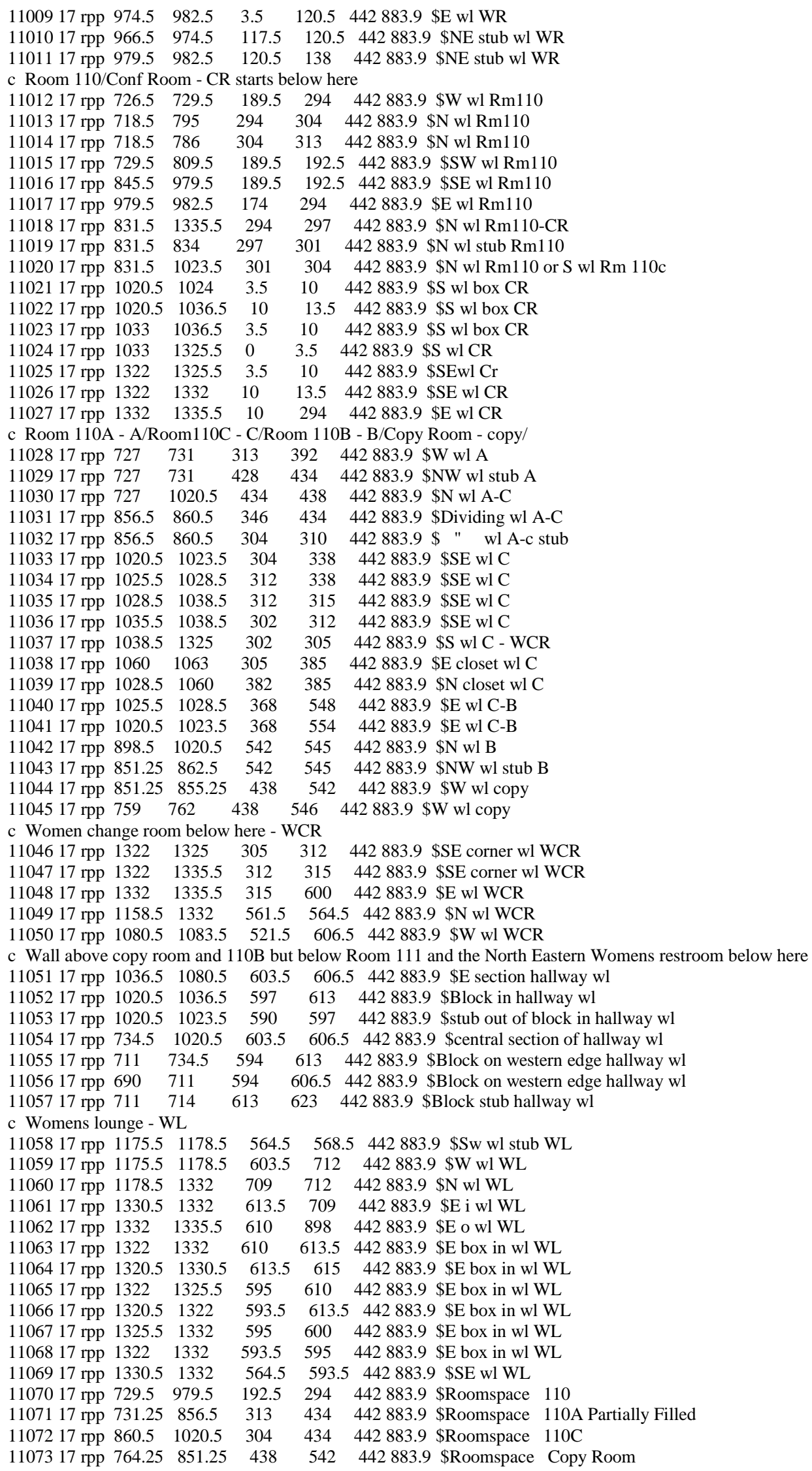




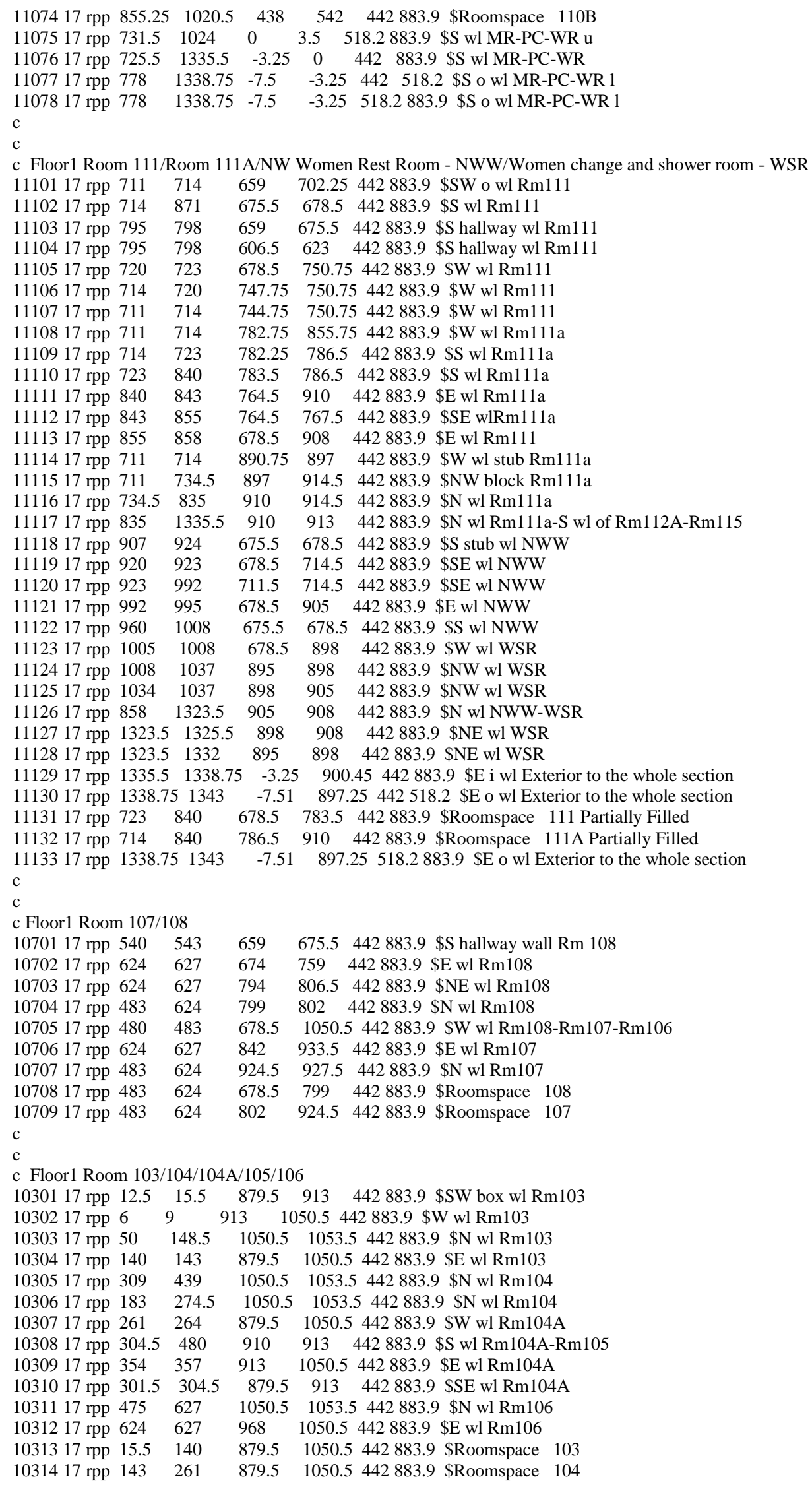




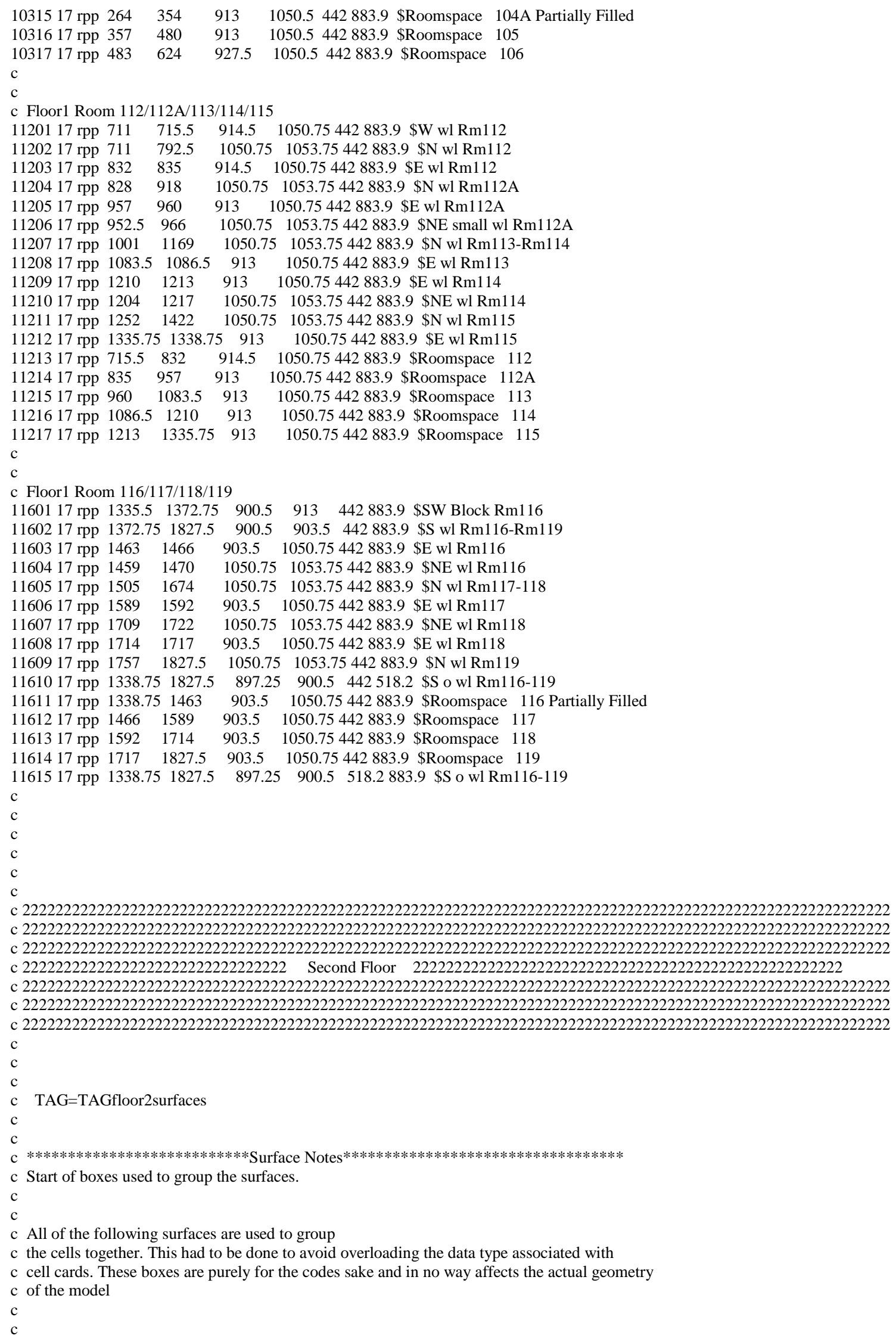


c Void box surrounding Second Floor

992 rpp -1000 4000 -3000 4000883.91498 .6 \$Void

c

c NW Section of Floor2

20324 rpp -7.25

20424 rpp $-7.25 \quad 180$

20524 rpp $180 \quad 515$

20124 rpp -10.251519

20624 rpp $180 \quad 515$

20724 rpp $515 \quad 840$

20824 rpp $515 \quad 959.5$

20924 rpp $840 \quad 1160$

21024 rpp $1160 \quad 1519$

21124 rpp 959.51519

-5 $\quad 190 \quad 883.9$ 1468.1 \$Cell box SW corner

$190 \quad 960 \quad 883.9$ 1468.1 \$Cell box W

$270 \quad 1020 \quad 883.91468 .1$ \$Cell box NW corner

-85.5 $1100 \quad 883.9$ 1468.1 \$NW Section Box

$\begin{array}{llll}190 & 270 & 883.9 & 1468.1\end{array}$ \$Cell box S1

-85.5 $600 \quad 883.9$ 1468.1 \$Cell box S2

$600 \quad 1079.75883 .91468 .1$ \$Cell box N

$\begin{array}{llll}-85.5 & 600 & 883.9 & 1468.1\end{array}$ \$Cell box SE

$\begin{array}{llll}-85.5 & 600 & 883.9 & 1468.1\end{array}$ \$Cell box SE2

$600 \quad 1079.75883 .9$ 1468.1 \$Cell box NE

c NE Section of Floor2

21221 rpp $0 \quad 820.25 \quad 0 \quad 1168.25883 .91468 .1$ \$Cell box NE Section

c S Section of Floor2

21322 rpp $-3 \quad 2343.5 \quad-15 \quad 210.25 \quad 883.91468 .1$ \$Cell box S Section

c E Section of Floor2

21423 rpp $-7 \quad 574 \quad-4 \quad 1548.5 \quad 883.9$ 1071.9 \$Cell box W Section

21523 rpp $574 \quad 1080 \quad 255 \quad 750 \quad 883.9$ 1071.9 \$Cell box E Section

21823 rpp $-7 \quad 590.5 \quad-4 \quad 1548.5 \quad 1071.91257 .3$ \$Cell box 3rd Floor W Section

C

C

C

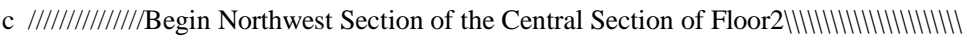

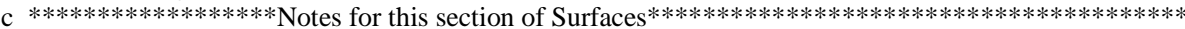

c The origin was set as the SW corner of the inside wall of Room 910

c Floor2 Room 910/911/hallway above these rooms

9100224 rpp $-7.25 \quad 0 \quad 00168 \quad 883.9$ 1468.1 \$W wl Rm910

9100324 rpp $-7.25 \quad 241 \quad-5 \quad 0 \quad 883.91468 .1$ \$S wl Rm910

$\begin{array}{lllllll}9100424 \text { rpp } & 0 & 81.5 & 149 & 154 & 883.9 & 1468.1\end{array}$ \$N wl Rm910

9100524 rpp $117.5 \quad 133 \quad 149 \quad 154 \quad 883.9$ 1468.1 \$NE wl Rm910

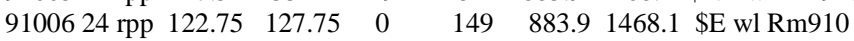

9100724 rpp $169 \quad 241 \quad 149 \quad 154 \quad 883.91468 .1 \quad \$ N$ wl

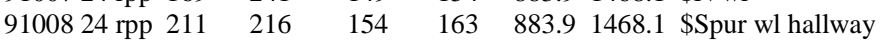

9100924 rpp $211 \quad 216 \quad 237 \quad 243.5 \quad 883.9 \quad 1468.1 \quad$ \$N Spur wl hallway

9101024 rpp $211 \quad 410 \quad 243.5 \quad 248.5 \quad 883.9 \quad 1468.1$ \$N boundary wl in hallway

9101124 rpp $-7.25 \quad 0 \quad 204 \quad 218 \quad 883.9 \quad 1468.1$ \$NW block in hallway

9101224 rpp $-10.25 \quad-7.25 \quad 204 \quad 1082.75$ 883.9 1468.1 \$W wl hallway-Rm912-914-916-Mech

9101324 rpp $0 \quad 122.75 \quad 0 \quad 149 \quad 883.91468 .1$ \$Roomspace 910

9101424 rpp $127.75241 \quad 0 \quad 149 \quad 883.91468 .1$ \$Roomspace 911

C

C

c Floor2 Room 912/914/916

$\begin{array}{lllllll}91201 & 24 \\ \text { rpp } & -7.25 & 151 & 218 & 223 & 883.9 & 1468.1\end{array}$ \$S wl Rm912

9120224 rpp $146 \quad 151 \quad 223 \quad 302 \quad 883.9$ 1468.1 $\$$ \$E wl Rm912

9120324 rpp $146 \quad 151 \quad 338 \quad 354 \quad 883.9$ 1468.1 \$NE wl Rm912

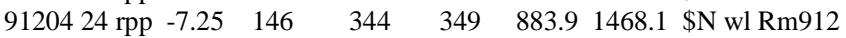

$\begin{array}{llllllll}91205 & 24 \\ \text { rpp } & 146 & 151 & 390 & 541 & 883.9 & 1468.1 & \text { \$E wl Rm } 914\end{array}$

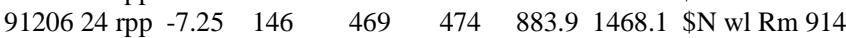

9120724 rpp $146 \quad 151 \quad 613 \quad 916 \quad 883.9$ 1468.1 \$E wl Rm916-Mech

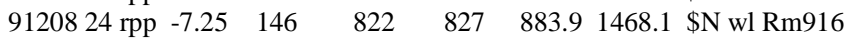

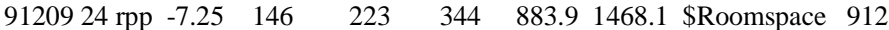

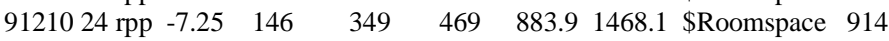

9121124 rpp $-7.25 \quad 146 \quad 474 \quad 822 \quad 883.91468 .1$ \$Roomspace 916

C

c Floor2 Room 913/915/928/929

9130124 rpp $211 \quad 216 \quad 299.5 \quad 539.5 \quad 883.9 \quad 1468.1$ \$W wl Rm913-915

9130224 rpp $216 \quad 221.5 \quad 299.5 \quad 304.5 \quad 883.9 \quad 1468.1$ \$SW stub wl Rm913 


\begin{tabular}{|c|c|c|c|}
\hline 9130324 грp 257.5 & 476 & 299.5 & 304.5883 .9 1468.1 \$S wl Rm913 928 \\
\hline 9130424 rpp 216 & 329 & 393.5 & 398.5883 .91468 .1 \$N wl Rm913 \\
\hline 9130524 rpp 329 & 334 & 304.5 & 883.9 1468.1 \$E wl Rm913-915 \\
\hline 9130624 rpp 291 & 296 & 358.5 & 883.9 1468.1 \$NE compartment Rm913 \\
\hline 9130724 rpp 296 & 329 & 358.5 & 883.91468 .1 \$NE compartment Rm913 \\
\hline 9130824 rpp 216 & 274 & 534.5 & 883.91468 .1 \$NW wl Rm915 \\
\hline 9130924 rpp 310 & 476 & 534.5 & 883.9 1468.1 \$NE wl Rm915-Rm929 \\
\hline 9131024 rpp 391 & 405 & 539.5 & 542.5883 .9 1468.1 \$Block in N wl Rm929 \\
\hline 9131124 rpp 334 & 471 & 417 & $422 \quad 883.91468 .1$ \$N wl Rm928 \\
\hline 9131224 rpp 471 & 476 & 304.5 & $346 \quad 883.9$ 1468.1 \$SE wl Rm928 \\
\hline 9131324 rpp 471 & 476 & 382 & 493.5883 .9 1468.1 \$NE wl Rm928 \\
\hline 9131424 rpp 471 & 476 & 529.5 & 534.5883 .9 1468.1 \$NE wl Rm929 \\
\hline 9131524 rpp 216 & 291 & 304.5 & 393.5883 .91468 .1 \$Roomspace 913 \\
\hline 9131624 rpp 216 & 329 & 398.5 & 534.5883 .91468 .1 \$Roomspace 915 \\
\hline 9131724 rpp 334 & 471 & 304.5 & $417 \quad 883.91468 .1$ \$Roomspace 928 \\
\hline 9131824 rpp 334 & 471 & 422 & 883.91468 .1 \$Roomspace 929 \\
\hline c & & & \\
\hline C & & & \\
\hline с $\quad$ Floor2 Room 9 & 3/919/91 & 7/921/905 & $5 / 923 /$ mech room \\
\hline 9180124 rpp 211 & 216 & 613.5 & 697.5883 .91468 .1 \$W wl Rm918 \\
\hline 9180224 rpp 211 & 216 & 733.5 & 749883.91468 .1 \$W wl Rm918 \\
\hline 9180324 rpp 211 & 216 & 785 & 883.91468 .1 \$W wl Rm917 \\
\hline 9180424 rpp 211 & 216 & 907.5 & 883.91468 .1 \$W wl Rm905 \\
\hline 9180524 rpp 151 & 168.5 & 911 & 883.91468 .1 \$NW hallway wl \\
\hline 9180624 rpp 204.5 & 211 & 911 & 883.91468 .1 \$NW hallway wl \\
\hline 9180724 rpp 500 & 505 & 613.5 & 883.91468 .1 \$E wl Rm919 \\
\hline 9180824 rpp 500 & 505 & 734 & 883.9 1468.1 \$E wl Rm 919 \\
\hline 9180924 rpp 500 & 505 & 785 & 883.9 1468.1 \$E wl Rm 921 \\
\hline 9181024 rpp 500 & 505 & 910 & 883.9 1468.1 \$E wl Rm 923 \\
\hline 9181124 rpp 216 & 500 & 739 & 883.91468 .1 \$N wl Rm919 \\
\hline 9181224 rpp 216 & 500 & 864 & 883.91468 .1 \$N wl Rm921 \\
\hline 9181324 rpp 216 & 500 & 988 & 993883.91468 .1 \$N wl Rm923 \\
\hline 9181424 rpp 355.5 & 360.5 & 618.5 & $739 \quad 883.9$ 1468.1 \$E wl Rm918 \\
\hline 9181524 rpp 355.5 & 360.5 & 744 & 883.9 1468.1 \$E wl Rm917 \\
\hline 9181624 rpp 355.5 & 360.5 & 869 & $988 \quad 883.9$ 1468.1 \$E wl Rm905 \\
\hline 9181724 rpp 381 & 395 & 817.75 & 827.25 883.9 1468.1 \$Block Rm921 \\
\hline 9181824 rpp 216 & 500 & 613.5 & 618.5883 .91468 .1 \$S wl Rm918 \\
\hline 9181924 rpp -7.25 & 552 & 1079.75 & $5 \quad 1082.75883 .91468 .1$ \$N wl mech rm \\
\hline 9182024 rpp 549 & 552 & 1072.75 & $5 \quad 1079.75883 .91468 .1 \quad \$ N$ wl mech rm \\
\hline 9182124 rpp 216 & 355.5 & 618.5 & $739 \quad 883.91468 .1$ \$Roomspace 918 \\
\hline 9182224 rpp 216 & 355.5 & 744 & 883.91468 .1 \$Roomspace 917 \\
\hline 9182324 rрp 216 & 355.5 & 869 & 883.91468 .1 \$Roomspace 905 \\
\hline 9182424 rpp 360.5 & 500 & 618.5 & 883.91468 .1 \$Roomspace 919 \\
\hline 9182524 rpp 360.5 & 500 & 744 & 883.91468 .1 \$Roomspace 921 \\
\hline 9182624 rpp 360.5 & 500 & 869 & 883.91468 .1 \$Roomspace 923 \\
\hline C & & & \\
\hline c & & & \\
\hline c $\quad$ Floor2 Room 927 & 7(Confer & ence Rool & om)/933/935/926/937/925/939 section contains some hallway remnants \\
\hline 9270124 rpp 469 & 474 & -10.5 & 248.5883 .91468 .1 \$W wl Rm927 \\
\hline 9270224 rpp 474 & 481 & 243.5 & 248.5883 .91468 .1 \$NW wl stub Rm927 \\
\hline 9270324 rpp 474 & 808 & -10.5 & -5.5883 .91468 .1 \$S wl Rm927 \\
\hline 9270424 rpp 666 & 671 & -5.5 & 534.5883 .91468 .1 \$E wl Rm927 \\
\hline 9270524 rpp 517 & 666 & 243.5 & 248.5883 .9 1468.1 \$NE wl Rm927 \\
\hline 9270624 rpp 796.75 & 808 & -5.5 & $7 \quad 883.91468 .1$ \$SE block Rm933 \\
\hline 9270724 rpp 808 & 813 & -85.5 & $82.5 \quad 883.9$ 1468.1 \$SE wl Rm933 \\
\hline 9270824 rpp 808 & 813 & 118.5 & 134.5883 .9 1468.1 \$NE wl Rm933 \\
\hline 9270924 rрp 671 & 808 & 124 & 129883.91468 .1 \$N wl Rm933 \\
\hline 9271024 rpp 808 & 813 & 170.5 & 346.5883 .9 1468.1 \$E wl Rm935 \\
\hline 9271124 rрp 795 & 808 & 235.25 & $256 \quad 883.9$ 1468.1 \$NE block Rm935 \\
\hline 9271224 rpp 671 & 808 & 2562 & 261883.91468 .1 \$N wl Rm935 \\
\hline 9271324 rpp 808 & 813 & 382.5 & $479.5 \quad 883.9$ 1468.1 \$NE wl Rm937 \\
\hline 9271424 rpp 671 & 808 & 388 & 393883.91468 .1 \$N wl Rm937 \\
\hline 9271524 rpp 524 & 529 & 381 & 883.91468 .1 \$W wl Rm926 \\
\hline 9271624 rрp 529 & 666 & 389 & 394883.91468 .1 \$N wl Rm926 \\
\hline 9271724 rpp 524 & 529 & 528 & 539.5883 .91468 .1 \$W wl Rm925 \\
\hline 9271824 rpp 529 & 718 & 534.5 & 539.5883 .91468 .1 \$N wl Rm925 \\
\hline 9271924 rрp 704.5 & 709.5 & 520.5 & 534.5883 .91468 .1 \$NE wl jog Rm939 \\
\hline 9272024 rpp 709.5 & 813 & 520.5 & 525.5883 .9 1468.1 \$NE wl jog Rm939 \\
\hline 9272124 rрp 808 & 813 & 515.5 & 520.5883 .9 1468.1 \$NE wl jog Rm939 \\
\hline 9272224 rpp 794.5 & 813 & 525.5 & 539.5883 .91468 .1 \$NE wl jog Rm939 \\
\hline
\end{tabular}




\begin{tabular}{|c|c|c|c|c|}
\hline \multicolumn{2}{|c|}{9272324 rpp 792.5} & 794.5 & 534.5 & 539.5883 .91468 .1 \$NE wl jog Rm939 \\
\hline 9272424 rpp & 405 & 410 & 202.5 & 243.5883 .91468 .1 \$Hallway wl W of Rm927 \\
\hline 9272524 rpp & 410 & 428 & 202.5 & 207.5883 .91468 .1 \$Hallway wl W of Rm927 \\
\hline 9272624 rpp & 464 & 469 & 202.5 & 207.5883 .91468 .1 \$Hallway wl W of Rm927 \\
\hline 9272724 rрp & 381 & 395 & 248.5 & 251.5883 .91468 .1 \$Hallway wl W of Rm927 \\
\hline 9272824 rрp & 474 & 666 & -5.5 & 243.5883 .91468 .1 \$Roomspace 927 \\
\hline 9272924 rрp & 529 & 666 & 248.5 & $389 \quad 883.91468 .1$ \$Roomspace 926 \\
\hline 9273024 rpp & 529 & 666 & 394 & 534.5883 .91468 .1 \$Roomspace 925 \\
\hline 9273124 rpp & 671 & 808 & -5.5 & 124883.9 1468.1 \$Roomspace 933 Room Partial Fill \\
\hline 9273224 rpp & 671 & 808 & 129 & 883.9 1468.1 \$Roomspace 935 Room Partial Fill \\
\hline 9273324 rрp & 671 & 808 & 261 & $388 \quad 883.91468 .1$ \$Roomspace 937 \\
\hline $9273424 \mathrm{rpp}$ & 671 & 808 & 393 & 520.5883 .91468 .1 \$Roomspace 939 Room Partial Fill \\
\hline C & & & & \\
\hline C & & & & \\
\hline c Floor2 Ro & pom 920 & 0/927/924 & 4/968/965 & $5 / 967 / 964$ \\
\hline 9200124 rpp & 552.5 & 557.5 & 613.5 & $698 \quad 883.91468 .1$ \$W wl Rm920 \\
\hline 9200224 rpp & 552.5 & 557.5 & 734 & $749 \quad 883.91468 .1$ \$W wl Rm920 \\
\hline 9200324 rpp & 552.5 & 557.5 & 785 & 883.91468 .1 \$W wl Rm927 \\
\hline 9200424 rpp & 552.5 & 557.5 & 910 & $988 \quad 883.9$ 1468.1 \$W wl Rm924 \\
\hline 9200524 rpp & 557.5 & 706 & 613.5 & 618.5883 .9 1468.1 \$S wl Rm920 \\
\hline 9200624 rpp & 695.5 & 700.5 & 618.5 & $863 \quad 883.91468 .1$ \$E wl Rm920 \\
\hline 9200724 rpp & 557.5 & 695.5 & 739 & $744 \quad 883.91468 .1$ \$N wl Rm920 \\
\hline 9200824 rpp & 557.5 & 706 & 863 & $868 \quad 883.91468 .1 \quad$ \$N wl Rm927 \\
\hline 9200924 rpp & 650 & 655 & 868 & 1079.75883 .91468 .1 \$E wl Rm924 \\
\hline 9201024 rpp & 546.75 & 650 & 988 & $993 \quad 883.91468 .1$ \$N wl Rm924 \\
\hline 9201124 rpp & 742 & 912 & 613.5 & 618.5883 .91468 .1 \$S wl Rm965 \\
\hline 9201224 rpp & 907 & 912 & 618.5 & $697 \quad 883.91468 .1$ \$E wl Rm965 \\
\hline 9201324 rpp & 813 & 907 & 738.5 & 743.5883 .91468 .1 \$N wl Rm965 \\
\hline 9201424 rpp & 907 & 912 & 733 & $749 \quad 883.9$ 1468.1 \$NE wl Rm965 \\
\hline 9201524 rpp & 907 & 912 & 785 & 883.91468 .1 \$E wl Rm967 \\
\hline 9201624 rpp & 813 & 914 & 865 & 883.91468 .1 \$N wl Rm967 \\
\hline 9201724 rpp & 700.5 & 808 & 791 & 883.91468 .1 \$N wl Rm968 \\
\hline 9201824 rpp & 748 & 753 & 796 & 883.91468 .1 \$Rm N of Rm968 \\
\hline 9201924 rpp & 742 & 765 & 863 & 883.91468 .1 \$S wl Rm964 \\
\hline 9202024 rpp & 760 & 765 & 868 & 883.91468 .1 \$S wl Rm964 \\
\hline 9202124 rpp & 765 & 808 & 902 & 907883.91468 .1 \$S wl Rm964 \\
\hline $9202224 \mathrm{rpp}$ & 655 & 954.5 & 1050 & 1055883.91468 .1 \$N wl Rm964 \\
\hline 9202324 rpp & 646 & 1676 & 1079.75 & $5 \quad 1082.75883 .91468 .1$ \$N o wl Rm964-Edge of section \\
\hline 9202424 rpp & 954.5 & 959.5 & 870 & 1079.75883 .91468 .1 \$E wl Rm964 \\
\hline $9202524 \mathrm{rpp}$ & 646 & 649 & 1072.75 & 1079.75 883.9 1468.1 \$NE o wl fragment Rm964 \\
\hline 9202624 rpp & 808 & 813 & 618.5 & 907883.91468 .1 \$E wl Rm968 \\
\hline $9202724 \mathrm{rpp}$ & 557.5 & 695.5 & 618.5 & $739 \quad 883.91468 .1$ \$Roomspace 920 \\
\hline 9202824 rpp & 557.5 & 695.5 & 744 & $863 \quad 883.91468 .1$ \$Roomspace 927 \\
\hline 9202924 rpp & 557.5 & 650 & 868 & 883.91468 .1 \$Roomspace 924 \\
\hline 9203024 rpp & 700.5 & 808 & 618.5 & $791 \quad 883.91468 .1$ \$Roomspace 968 \\
\hline 9203124 rpp & 813 & 907 & 618.5 & 738.5883 .91468 .1 \$Roomspace 965 \\
\hline 9203224 rpp & 813 & 907 & 743.5 & $865 \quad 883.91468 .1$ \$Roomspace 967 \\
\hline 9203324 rpp & 655 & 954.5 & 907 & 1050883.91468 .1 \$Roomspace 964-Conf. Room Partial Fil \\
\hline C & & & & \\
\hline $\mathrm{C}$ & & & & \\
\hline Floor2 Ro & jom 930 & 0/932/934 & 4/936/938 & 8/941/943/945/947/949 \\
\hline 9300124 rpp & 813 & 819 & -85.5 & -80.5 883.9 1468.1 \$SW hallway wl stub W of Rm930 \\
\hline 9300224 rpp & 854.5 & 1145.5 & -85.5 & -80.5 883.9 1468.1 \$S wl Rm930 \\
\hline 9300324 rрp & 861 & 866 & -80.5 & -5.5 883.91468 .1 \$W wl Rm930 \\
\hline $9300424 \mathrm{rpp}$ & 861 & 866 & 30.5 & 119.5883 .91468 .1 \$W wl Rm930 \\
\hline 9300524 rpp & 861 & 866 & 155.5 & 171.5883 .91468 .1 \$W wl Rm932 \\
\hline $9300624 \mathrm{rpp}$ & 861 & 866 & 207.5 & 368.5883 .91468 .1 \$W wl Rm934 \\
\hline 9300724 rpp & 861 & 866 & 404.5 & 420.5883 .91468 .1 \$W wl Rm936 \\
\hline 9300824 rpp & 861 & 866 & 456.5 & 539.5883 .91468 .1 \$W wl Rm938 \\
\hline 9300924 rpp & 866 & 1116.5 & 37.75 & 42.75883 .91468 .1 \$N wl Rm930-941 \\
\hline $9301024 \mathrm{rpp}$ & 866 & 1116.5 & 161 & $166 \quad 883.91468 .1$ \$N wl Rm932-943 \\
\hline 9301124 rpp & 866 & 1116.5 & 285.5 & 290.5883 .91468 .1 \$N wl Rm934-945 \\
\hline 9301224 rpp & 866 & 1116.5 & 410 & $415 \quad 883.91468 .1 \quad$ \$N wl Rm936-947 \\
\hline 9301324 rpp & 866 & 1116.5 & 534.5 & 539.5883 .91468 .1 \$N wl Rm938-949 \\
\hline 9301424 rpp & 988.75 & 993.75 & -80.5 & 37.75883 .91468 .1 \$E wl Rm930 \\
\hline 9301524 rpp & 988.75 & 993.75 & 42.75 & $161 \quad 883.91468 .1$ \$E wl Rm932 \\
\hline 9301624 rpp & 988.75 & 993.75 & 166 & 883.91468 .1 \$E wl Rm934 \\
\hline 9301724 rpp & 988.75 & 993.75 & 290.5 & 883.91468 .1 \$E wl Rm936 \\
\hline 9301824 rpp & 988.75 & 993.75 & 415 & 534.5883 .91468 .1 \$E wl Rm938 \\
\hline 9301924 rpp & 1116.5 & 1121.5 & -80.5 & -49.5 883.9 1468.1 \$E wl Rm941 \\
\hline
\end{tabular}




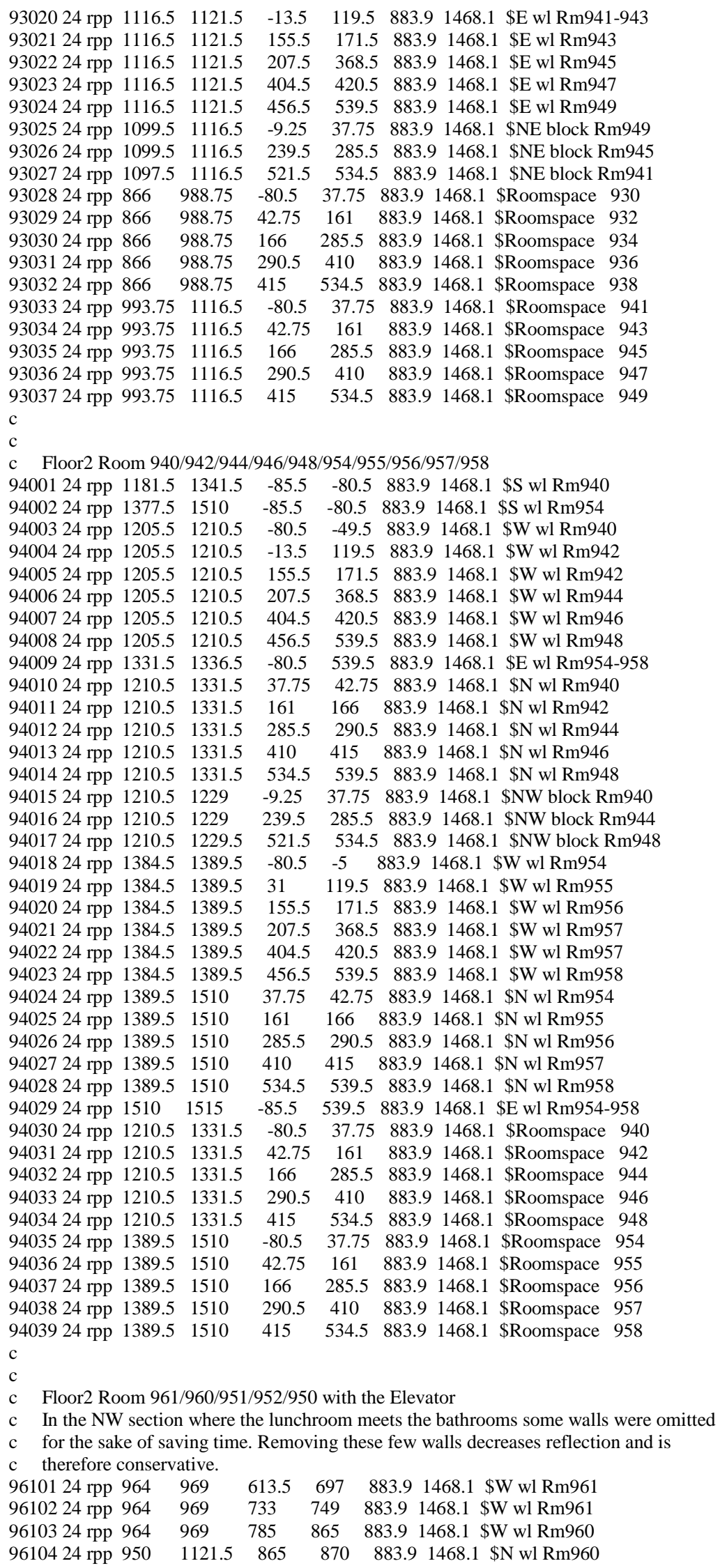




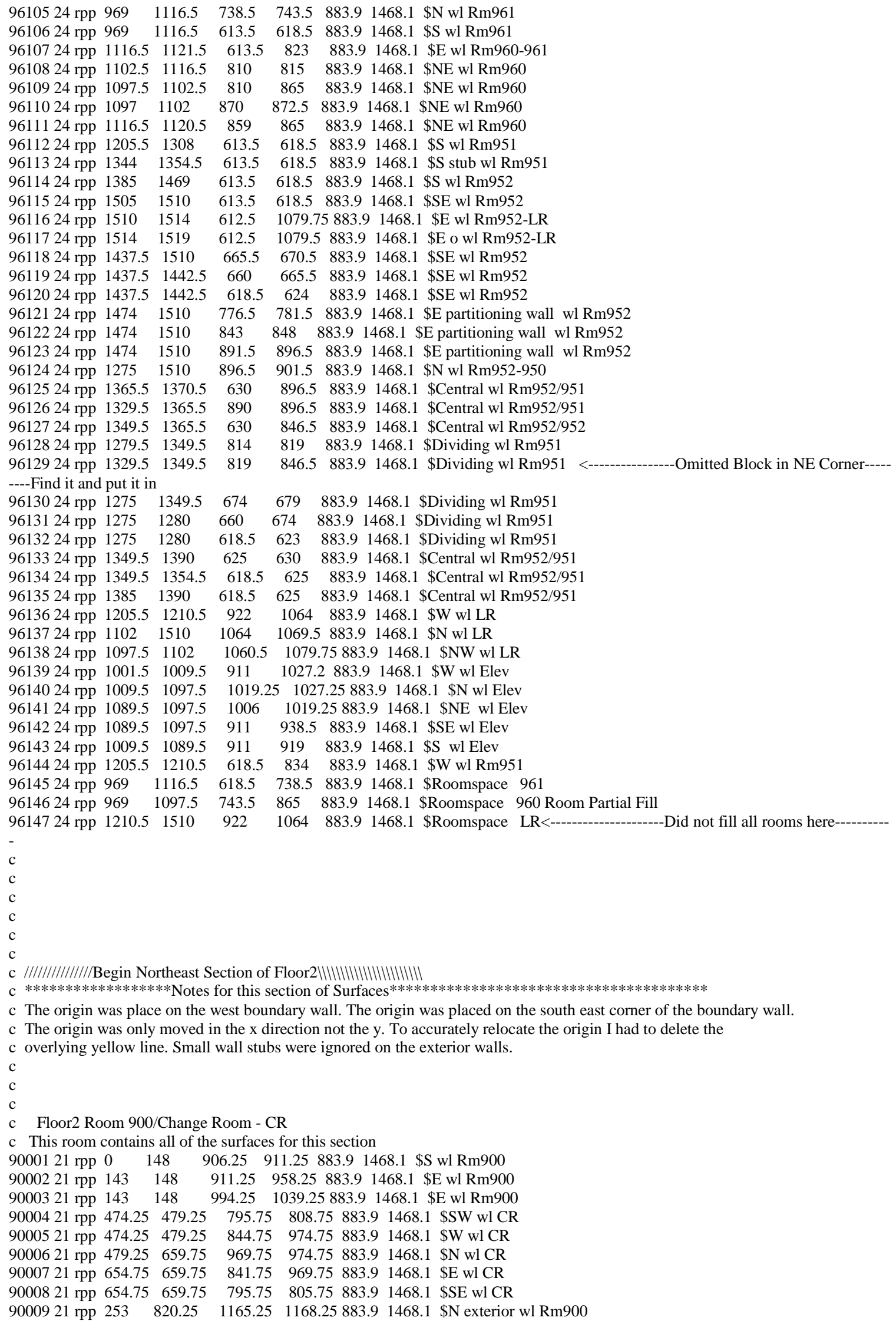




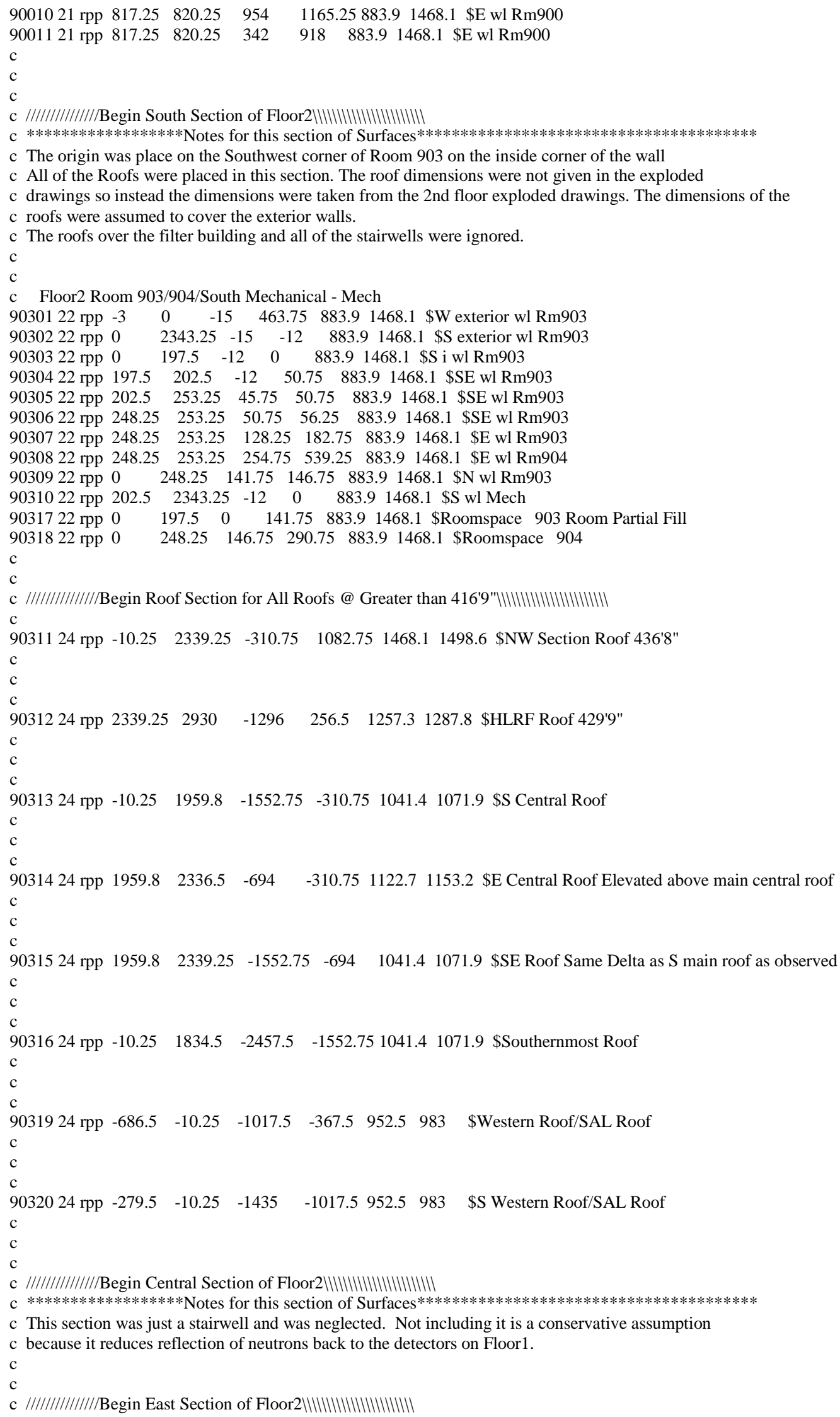




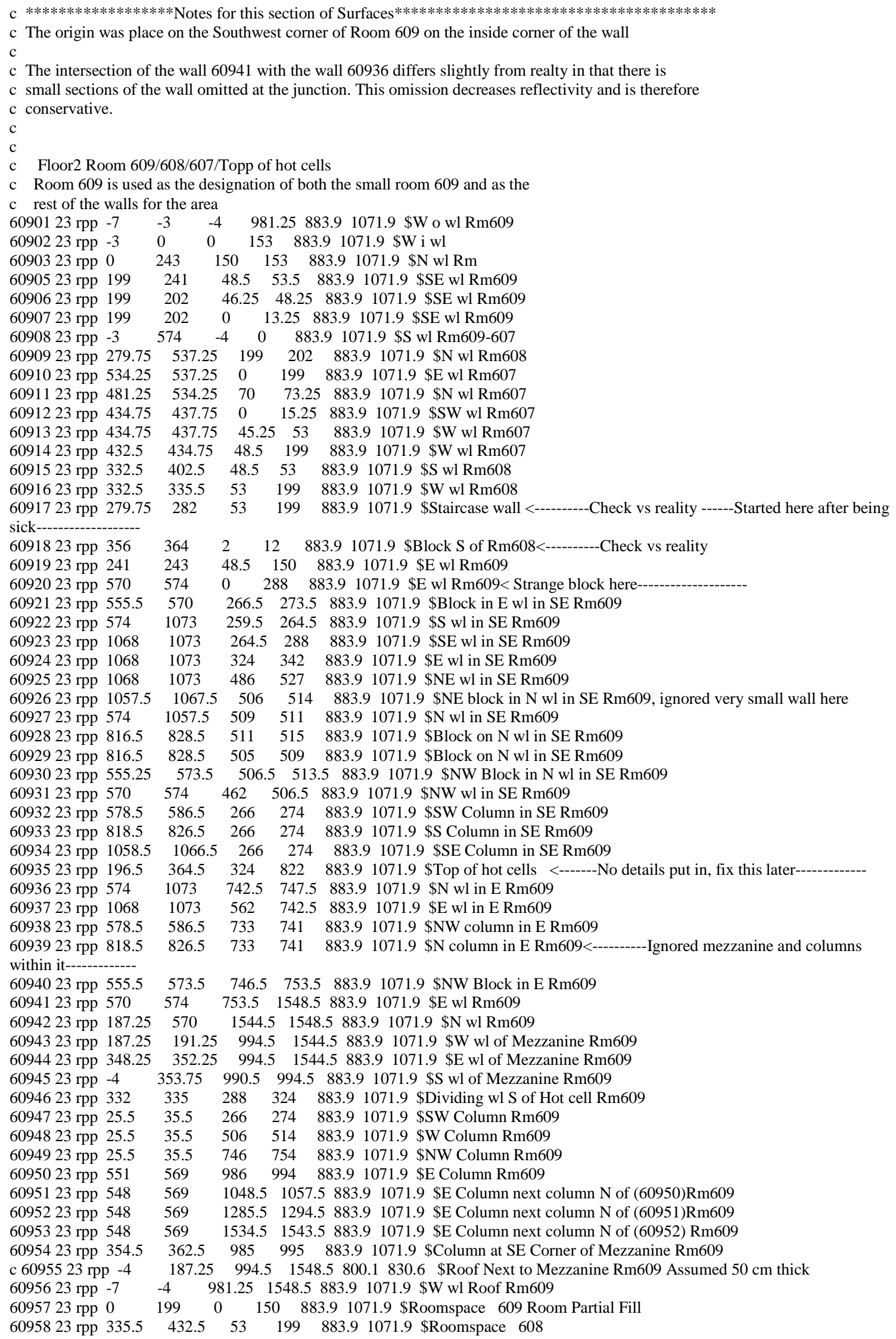




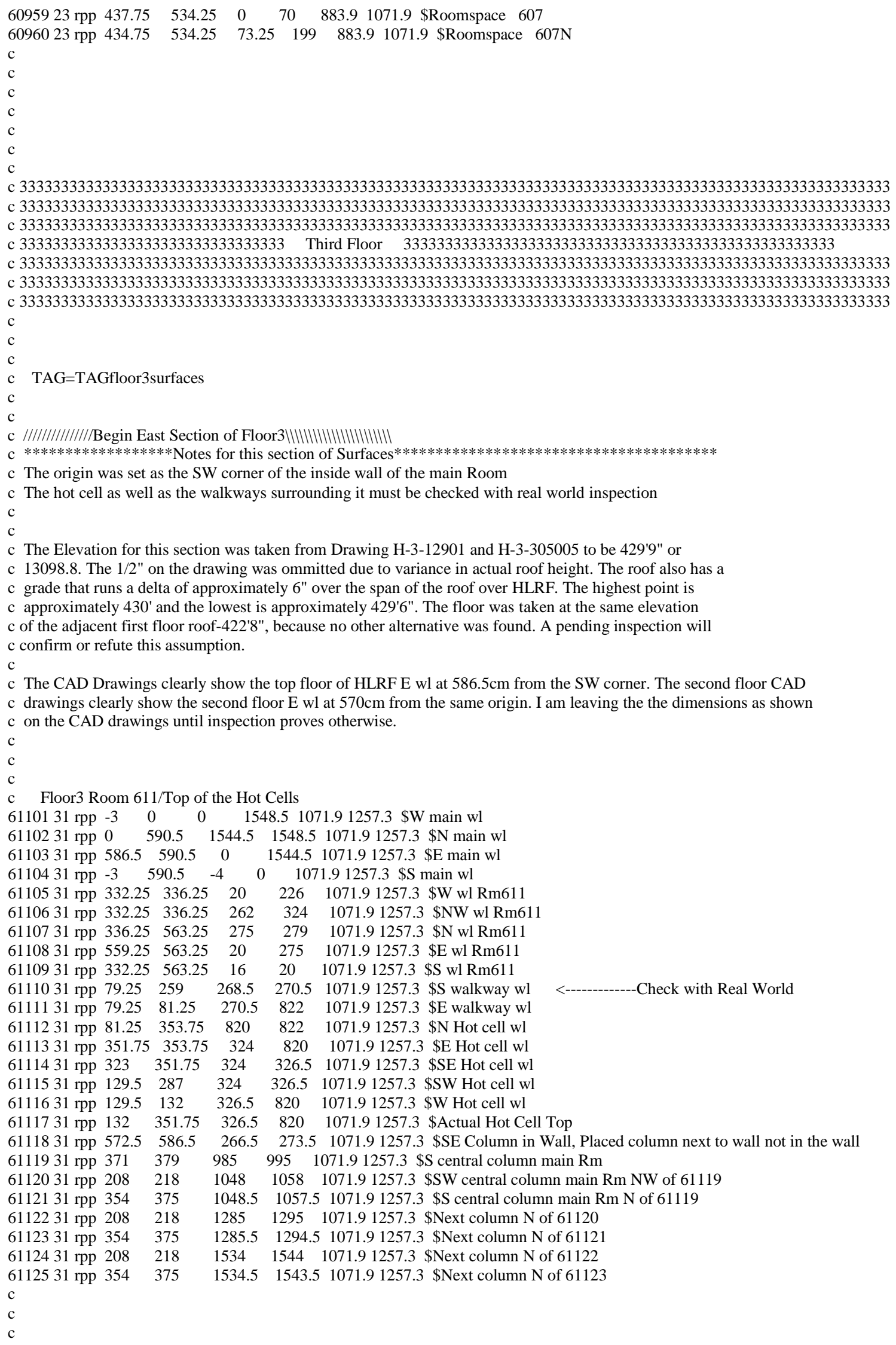




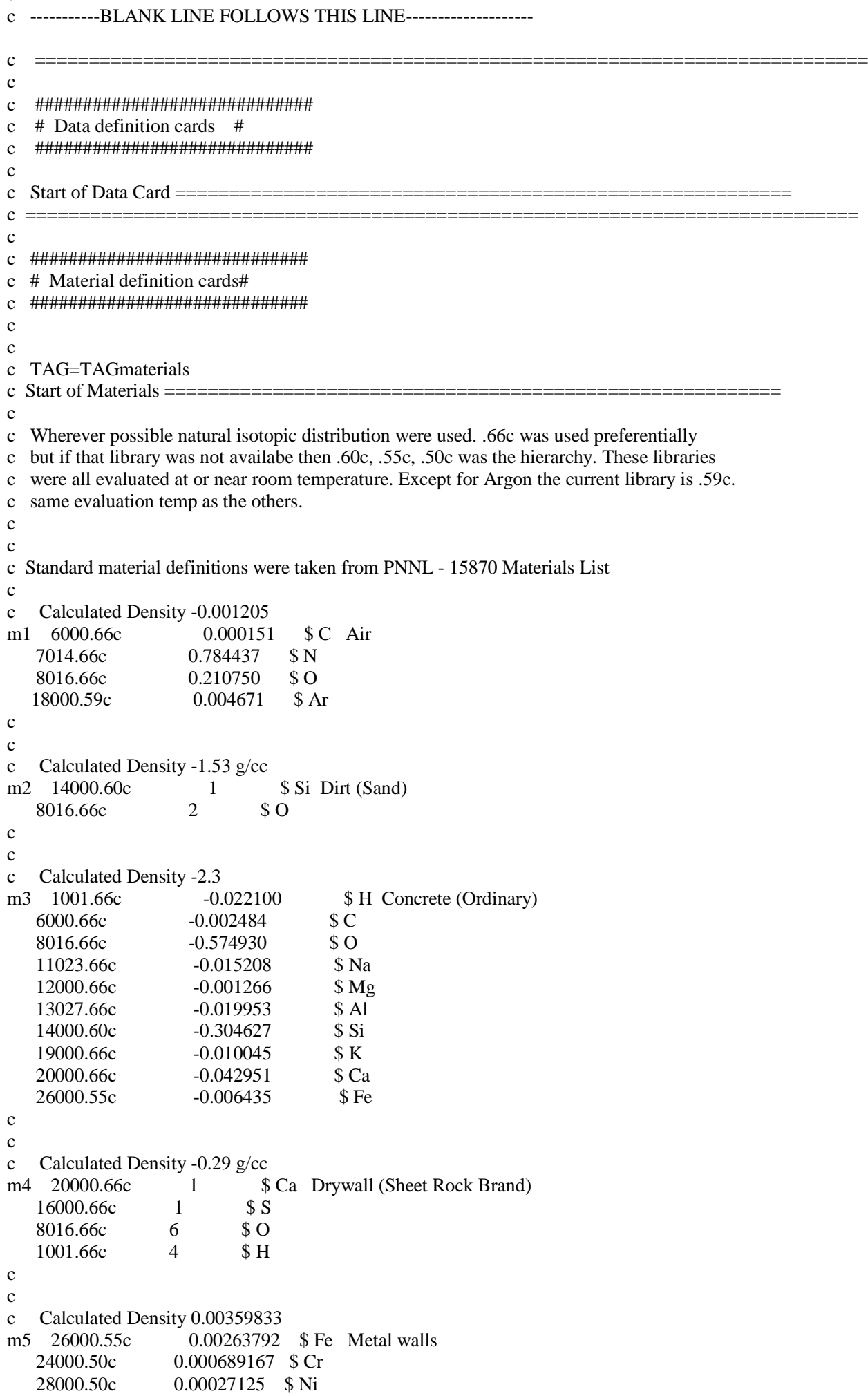




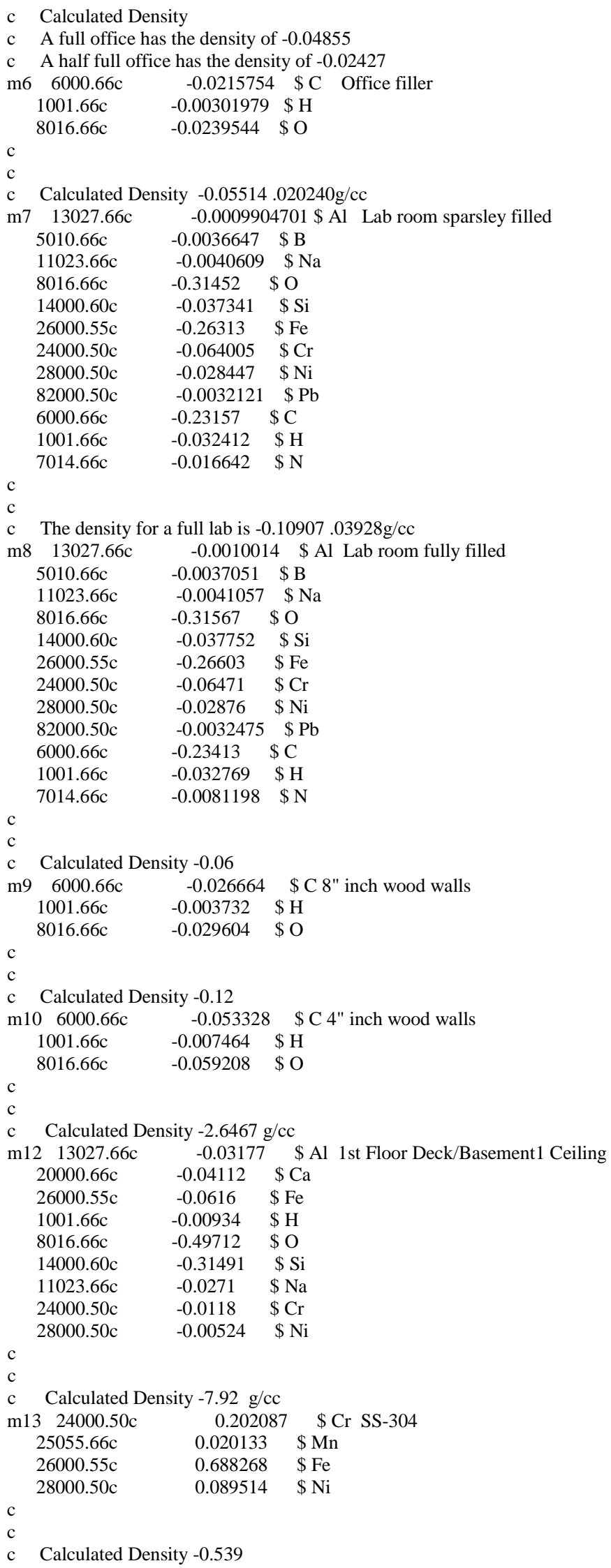




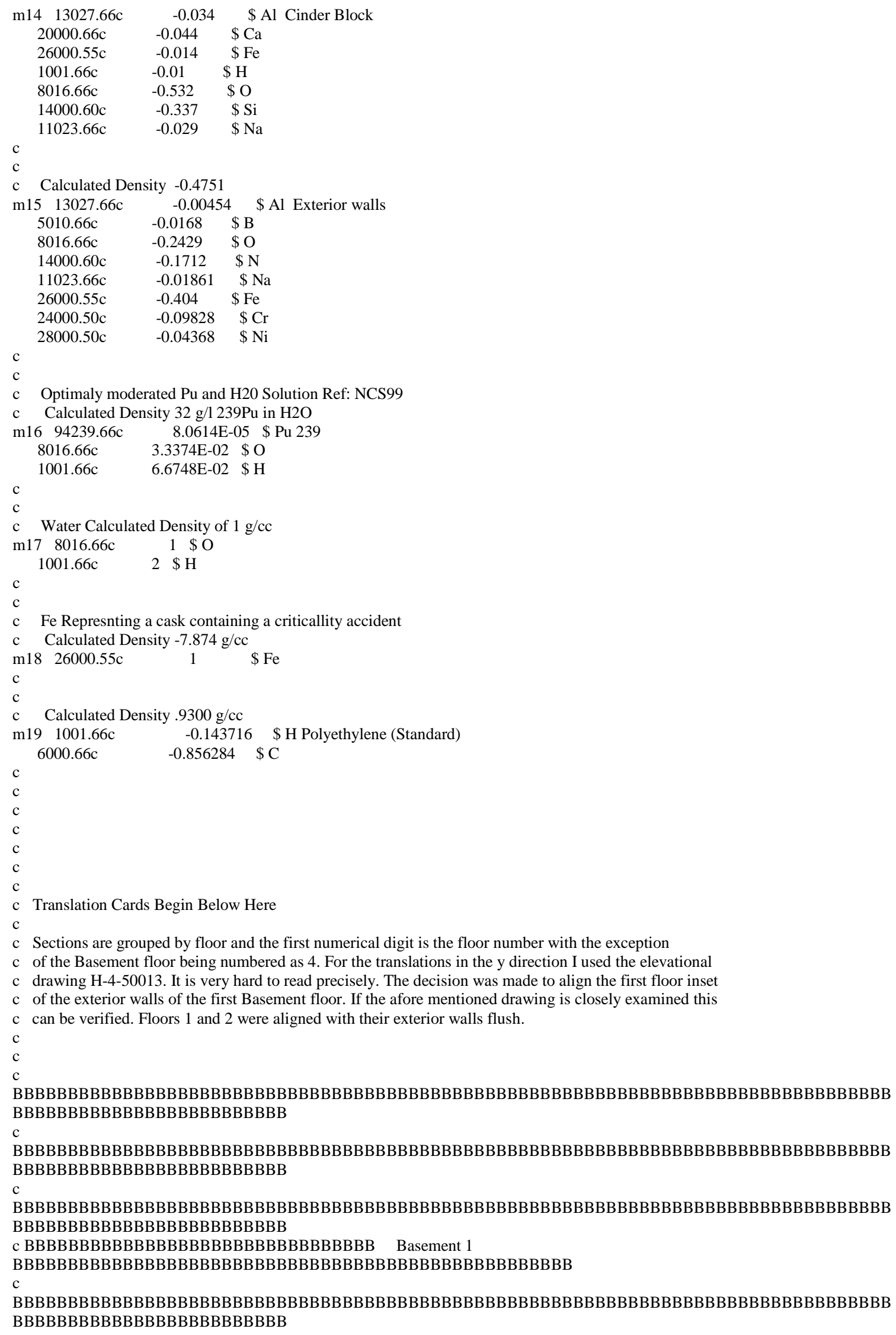




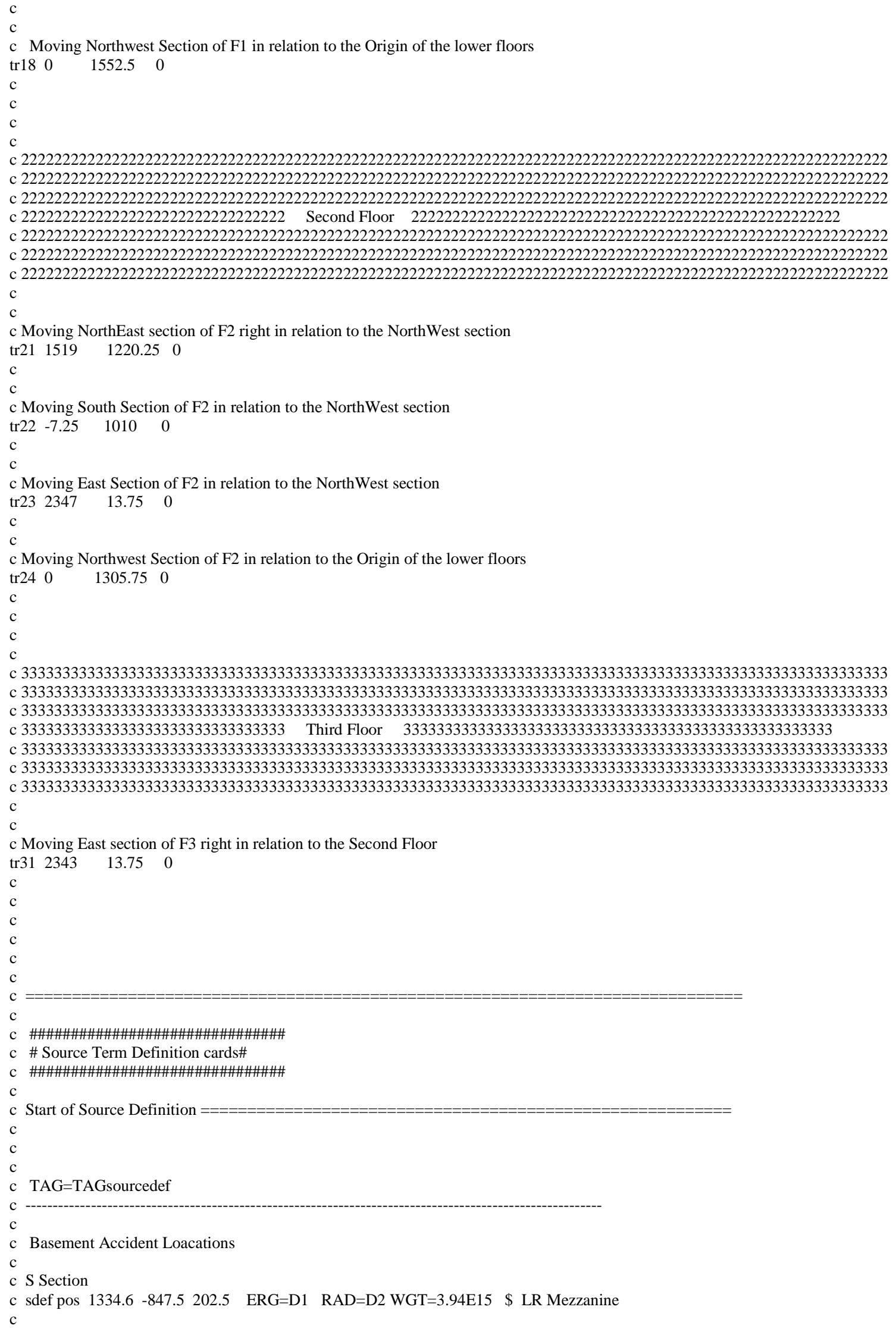




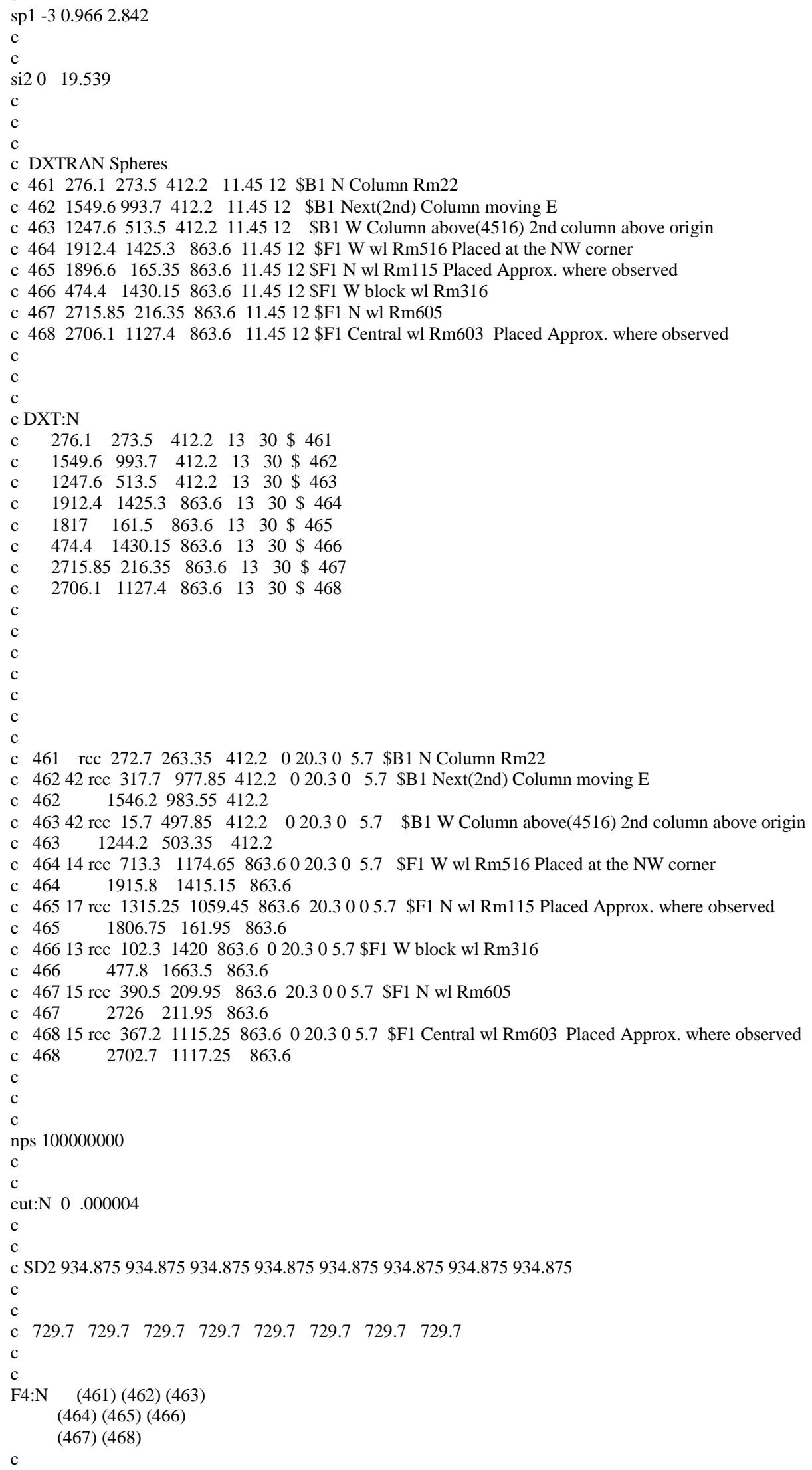

C

461 rcc $272.7263 .35 \quad 412.2 \quad 020.305 .7$ \$B1 N Column Rm22

c 46242 rcc $317.7 \quad 977.85412 .2 \quad 020.30 \quad 5.7$ \$B1 Next(2nd) Column moving E

c $462 \quad 1546.2983 .55412 .2$

c 46342 rcc $15.7497 .85 \quad 412.2 \quad 020.30 \quad 5.7 \quad$ \$B1 W Column above(4516) 2nd column above origin

$\begin{array}{llll}\text { c } 463 \quad 1244.2 & 503.35 & 412.2\end{array}$

c 46414 rcc $713.31174 .65863 .6020 .305 .7 \quad$ \$F1 W wl Rm516 Placed at the NW corner

$\begin{array}{lllll}\text { C } & 464 & 1915.8 & 1415.15 \quad 863.6\end{array}$

c 46517 rcc 1315.251059 .45863 .620 .3005 .7 \$F1 N wl Rm115 Placed Approx. where observed

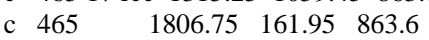

c 46613 rcc 102.31420863 .6020 .305 .7 \$F1 W block wl Rm316

$\begin{array}{llll}\text { C } 466 \quad 477.8 & 1663.5 \quad 863.6\end{array}$

c 46715 rcc 390.5209 .95863 .620 .3005 .7 \$F1 N wl Rm605

c $467 \quad 2726 \quad 211.95 \quad 863.6$

c 46815 rcc 367.21115 .25863 .6020 .305 .7 \$F1 Central wl Rm603 Placed Approx. where observed 
FC4 461=B1 Rm22, 462=B1 N of Rm34, 463=W of Rm35, 464=F1 W of Rm516,

465=F1 N of Rm115, 466=F1 W of Rm316, 467=F1 N of Rm605, 468=F1 E of 604

c

C

E4 4E-6 20

MCNP Input Modeled by Bryce Greenfield

c Listing for NIST 3" dry sphere; detailed src, sphere \& detectors, Table 24

C

с 34567890123456789112345678921234567893123456789412345678951234567896123456789712345678

c

C

C

c Cell Records

c Fission Chamber Except Spaces, $+\mathrm{Z}$

$16-21.45-12$-3 imp:n=1 \$1,Pt

$25-2.5811-46-5$ imp:n=1 \$2,Al reduced for smearing

$35-2.77-16-2$ imp:n=1 \$3,Al

48 - 2.28 -9 10 -6 imp:n=1 \$4,KEL-F

$5 \quad 5-2.7 \quad 11-12 \quad 13-10$ imp:n=1 \$5,Al

$65-2.714-1517-16$ imp:n=1 \$6,Al

7 5 -2.7 -18 19 -13 imp:n=1 \$7,Al

8 - 8 - 2.220 -21 22 -19 imp:n=1 \$8,KEL-F

$98-2.223-2119-24$ imp:n=1 \$9,KEL-F

$108-2.225-43-26$ imp:n=1 \$10,KEL-F

118 -2.2 $27-426-28$ imp:n=1 \$11,KEL-F

$125-2.736-2726-29$ imp:n=1 \$12,Al

$135-2.730-3631-32$ imp:n=1 \$13,Al

148 -2.2 33 -27 34 -35 imp:n=1 \$14,KEL-F

$158-2.04627-437-35$ imp:n=1 \$15,KEL-F reduced for smearing

$165-2.7-2729-34$ imp:n=1 \$16,Al

$175-2.738-3940-41$ imp:n=1 \$21,Al

185 - $2.7-3942-43$ imp:n=1 \$22,Al

195 - 2.74 -38 46 -42 imp:n=1 \$24,Al

$205-2.7-4 \quad 46-47$ imp:n=1 \$25,Al

c Fission Chamber Except Spaces, $-Z$

$216-21.45-5153-52$ imp:n=1 \$1,Pt

225 -2.581 51 -54 55 -56 imp:n=1 \$2,Al reduced for smearing

$235-2.757-5152-56$ imp:n=1 \$3,Al

248 -2.2 58 -59 56 -60 imp:n=1 \$4,KEL-F

$255-2.761-6260-63$ imp:n=1 \$5,Al

265 -2.7 $64-6566-67$ imp:n=1 \$6,Al

275 - $2.7-6863-69$ imp:n=1 \$7,Al

288 -2.2 70 -71 69 -72 imp:n=1 \$8,KEL-F

298 -2.2 $73-7174-69$ imp:n=1 \$9,KEL-F

$308-2.275-5476-53$ imp:n=1 \$10,KEL-F

318 -2.2 77 -54 78 -76 imp:n=1 \$11,KEL-F

$325-2.786-7779-76$ imp:n=1 \$12,Al

$335-2.780-8682-81$ imp:n=1 \$13,Al

348 -2.2 83 -77 85 -84 imp:n=1 \$14,KEL-F

358 -2.046 77 -54 85 -87 imp:n=1 \$15,KEL-F reduced for smearing

365 -2.7 -77 84 -79 imp:n=1 \$16,Al

$375-2.788-8991-90$ imp:n=1 \$21,Al

$385-2.7-8993-92$ imp:n=1 \$22,Al

$395-2.754-8892-96$ imp:n=1 \$24,Al 
405 -2.7 -54 97 -96 imp:n=1 \$25,Al

c Source, sphere, supports

$517-1.85-101102-103$ imp:n=1 \$Cf

$5211-2.0-104103-105$ imp:n=1 \$A

$535-2.7104-106102-105$ imp:n=1 \$B

$545-2.7-106107-102$ imp:n=1 \$C

$552-7.92108-109107-105$ imp:n=1 \$D

$562-7.92-109110-107$ imp:n=1 \$E

$572-7.92111-109105-112$ imp:n=1 \$F

58 2 $-7.92-109112-113$ imp:n=1 \$G

$592-7.92-114113-115$ imp:n=1 \$H

$602-7.92116-109113-117$ imp:n=1 \$I

$612-7.92114-118120-119$ imp:n=1 \$J

$622-7.92121-106119-122$ imp:n=1 \$K

$632-7.92123-106122-124$ imp:n=1 \$L

$642-7.92123-125124-126$ imp:n=1 \$M

$652-7.92123-127126-128$ imp:n=1 \$N

$662-7.92-127128-129$ imp:n=1 \$O

$672-7.92-104129-130$ imp:n=1 \$P

68 2 2 -2.1 -131 -146 130 imp:n=1 \$Q Chain

$691-0.001185114-121119-115$ imp:n=1 \$R, water or air

$701-0.001185-121115-122$ imp:n=1 \$S, water or air

$711-0.001185-123122-124$ imp:n=1 \$T, water or air

725 -2.7 141 -142 145 -146 imp:n=1 \$U stem support ring

755 -2.7 38 -152 154 -146 imp:n=1 \$stem,+Z

765 -2.7 38 -151 154 -146 imp:n=1 \$stem,-Z

792 -7.92 158 -159 -124 imp:n=1 \$SS shell

c Spaces

c air cells above water line

1051 -0.001185 -141 129 -146 (131:-130) 104151152 imp:n=1 \$ around src shaft above O

$1061-0.001185-141124-129$ (125:126) 127151152 imp:n=1 \$ around src shaft below P

$1071-0.001185-123124-128$ imp:n=1 \$ just above T in central tube

1081 -0.001185 -149 141124 -146 (-141:142:-145) imp:n=1 \$ away from src shaft

C

c air cells below water line

$1091-0.001185148-12496-46-149159$

imp:n=1 \$ outside SS sphere, inside detector Zs

$1101-0.001185148-12446-149$ (38:43) (-40:39:43)

c inner face

(152:-38:-154) imp:n=1 $\$$ beyond $+Z$ detector

111 -0.001185 148 -124-149 -96 (38:-93) (90:39:-93)

c inner face

(151:-38:-154) imp:n=1 \$ beyond -Z detector

c water or air cells inside sphere but outside source+holder

$1121-0.001185-116114113-117$ imp:n=1 \$ annular recess at top of src capsule

1131 -0.001185 -158 -120 (109:-110:117) (114:-113) imp:n=1 \$ below src holder

$1141-0.001185-158120-124$ (118:119) 106 imp:n=1 \$ at src holder elevations

c air in source capsule

$1151-0.001185-104101102-103$ imp:n=1 \$ gap immediately around Cf

1161 -0.001185 -108 $106107-105$ imp:n=1 \$ gap immediately inside src capsule wall D

$1171-0.001185-111105-112$ imp:n=1 \$ gap inside of $\mathrm{F}$ in source capsule

c $\mathrm{P}-10$ gas in $+\mathrm{Z}$ detector

1210 -4 47 -19 (-20:-22) imp:n=1 \$ before collector B

1220 -4 12 24 -10 imp:n=1 \$ gap outboard of Item 5

1230 -11 813 -10 (-14:-17:16) imp:n=1 \$ around Item 6

1240 -8 13 -2 (-7:-6) imp:n=1 \$ between collector B and Pt plate

1250 -4 2728 -37 imp:n=1 \$ gap outboard of Item 12

1260 -36 2526 -29 (-30:-31:32) imp:n=1 \$ around Item 13

1270 -25 3 -29 imp:n=1 \$ before collector B

128 -4 $34-41$ (-33:35) imp:n=1 \$ between collector B and Pt plate

c $\mathrm{P}-10$ gas in $-\mathrm{Z}$ detector

1310 -4 69 -97 (-20:72) imp:n=1 \$ before collector B

1320 -4 12 60 -74 imp:n=1 \$ gap outboard of Item 5

1330 -11 860 -63 (-14:-66:67) imp:n=1 \$ around Item 6

1340 -8 52 -63 (-7:56) imp:n=1 \$ between collector B and Pt plate

1350 -4 $2787-78$ imp:n=1 \$ gap outboard of Item 12

1360 -36 2579 -76 (-30:-82:81) imp:n=1 \$ around Item 13

$1370-2579-53$ imp:n=1 \$ before collector B

1380 -4 91 -84 (-33:-85) imp:n=1 \$ between collector B and Pt plate 
1990 149:-148:146 imp:n=0 \$ external to model

c Surface Records

c Fission Chamber $+\mathrm{Z}$

$1 \mathrm{cz} 0.953$

$2 \mathrm{pz} 7.606$

3 pz 7.634

4 cz 1.194

5 pz 7.634

$6 \mathrm{pz} 7.558$

7 cz 0.921

8 cz 1.021

9 cz 1.194

10 pz 7.507

$11 \mathrm{cz} 1.119$

12 cz 1.157

13 pz 7.126

14 cz 1.081

$15 \mathrm{cz} 1.119$

16 pz 7.471

$17 \mathrm{pz} 7.253$

18 cz 1.157

$19 \mathrm{pz} 7.113$

20 cz 0.882

$21 \mathrm{cz} 1.194$

22 pz 7.075

$23 \mathrm{cz} 1.157$

24 pz 7.329

$25 \mathrm{cz} 0.858$

26 pz 7.710

27 cz 1.150

28 pz 7.837

29 pz 8.091

30 cz 1.074

$31 \mathrm{pz} 7.746$

32 pz 7.964

33 cz 0.874

34 pz 8.104

35 pz 8.142

36 cz 1.112

37 pz 7.888

38 cz 1.245

39 cz 1.786

40 pz 8.435

41 pz 8.529

42 pz 8.529

43 pz 8.583

$46 \mathrm{pz} 7.005$

47 pz 7.056

c Fission Chamber -Z

51 cz 0.953

$52 \mathrm{pz}-7.606$

$53 \mathrm{pz}-7.634$

54 cz 1.194

$55 \mathrm{pz}-7.634$

$56 \mathrm{pz}-7.558$

57 cz 0.921

58 cz 1.021

59 cz 1.194

$60 \mathrm{pz}-7.507$

61 cz 1.119

62 cz 1.157

$63 \mathrm{pz}-7.126$

64 cz 1.081

65 cz 1.119

$66 \mathrm{pz}-7.471$

$67 \mathrm{pz}-7.253$

68 cz 1.157

$69 \mathrm{pz}-7.113$ 
70 cz 0.882

$71 \mathrm{cz} 1.194$

$72 \mathrm{pz}-7.075$

$73 \mathrm{cz} 1.157$

$74 \mathrm{pz}-7.329$

75 cz 0.858

$76 \mathrm{pz}-7.710$

$77 \mathrm{cz} 1.150$

$78 \mathrm{pz}-7.837$

$79 \mathrm{pz}-8.091$

80 cz 1.074

$81 \mathrm{pz}-7.746$

$82 \mathrm{pz}-7.964$

83 cz 0.874

$84 \mathrm{pz}-8.104$

$85 \mathrm{pz}-8.142$

86 cz 1.112

$87 \mathrm{pz}-7.888$

$88 \mathrm{cz} 1.245$

89 cz 1.786

$90 \mathrm{pz}-8.435$

$91 \mathrm{pz}-8.529$

$92 \mathrm{pz}-8.529$

$93 \mathrm{pz}-8.583$

$96 \mathrm{pz}-7.005$

$97 \mathrm{pz}-7.056$

c

c source, holder

101 су 0.1

102 py -0.04

103 py 0.04

104 cy 0.127

105 py 0.506

106 cy 0.318

107 py -0.154

108 су 0.330

109 су 0.381

110 py -0.205

111 cy 0.152

112 py 0.563

113 py 0.608

114 cy 0.114

115 py 2.289

116 су 0.277

117 py 0.659

c Holder

118 cy 0.366

119 py 2.108

120 py 1.613

121 су 0.254

122 py 2.616

123 cy 0.3

124 py 3.753

125 cy 1.27

126 py 3.879

127 cy 0.445

128 py 4.133

129 py 4.260

130 py 4.895

131 су 0.159

132 py 79.0

c

141 cy 7.858

142 су 8.493

143 cy 14.446

144 cy 20.010

145 py 11.43

146 py 11.667

148 py -11.667

149 cy 11.667 


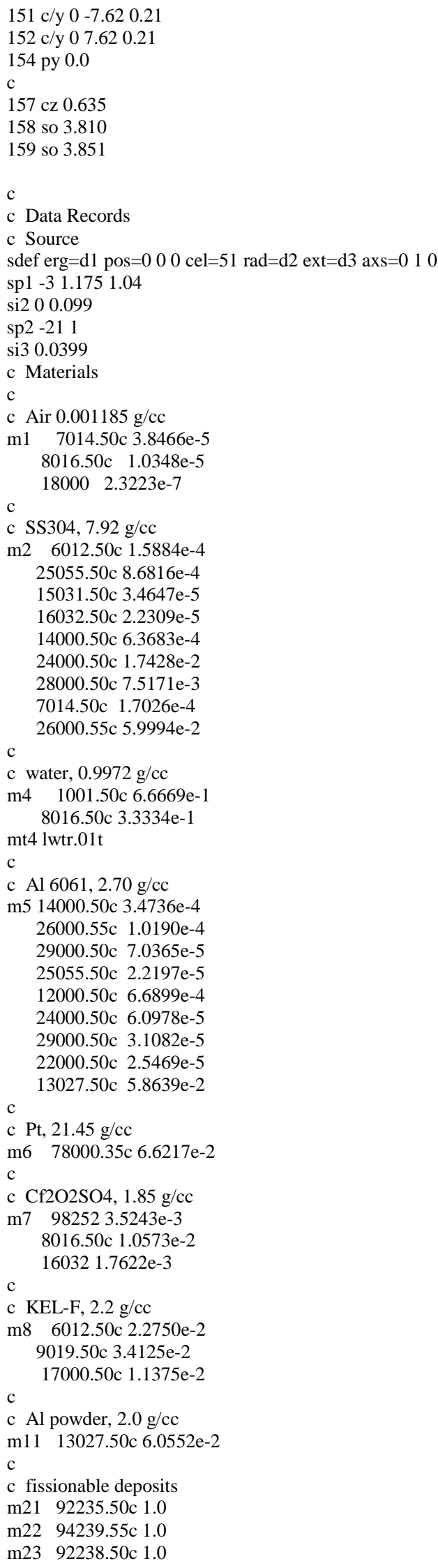




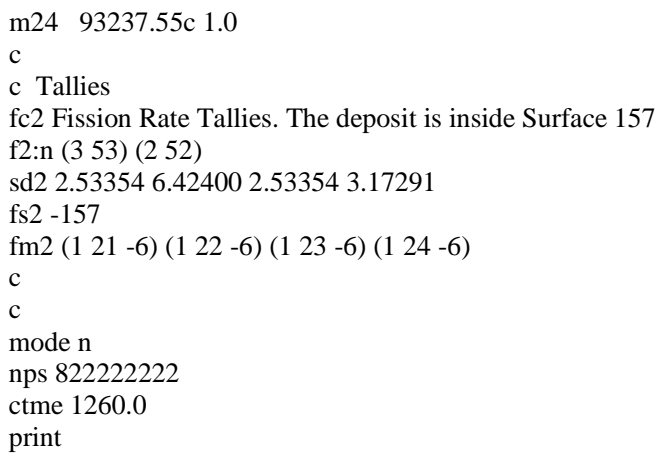

c Scenario 1: A Bare Sphere

c Surface cards

c 1 so 19.54 \$ $\mathrm{H} 20$ and Pu Sphere 
c c Scenario 3: A Metal Shielded Sphere

$\mathrm{C} C$

c c Surface cards

c 1 so 19.54 \$ H20 and Pu Sphere

c 2 so $219.04 \$ .50 \mathrm{~cm}$ less than $2 \mathrm{~m}$

c 3 so $220.04 \$ .50 \mathrm{~cm}$ greater than $2 \mathrm{~m}$

c 4 so 31.54 \$ Fe Reflector

c

C

c Blank line Follows

c Data cards

C

c Materials Cards

c Optimaly moderated Pu and H20 Solution Ref: NCS99

c Calculated Density $32 \mathrm{~g} / \mathrm{l} 239 \mathrm{Pu}$ in H2O

m1 plib $=04$ p nlib $=50 \mathrm{~m}$ pnlib $=24 \mathrm{u}$

94239.60c 8.0614E-05 \$ Pu 239

$1001.50 \mathrm{~m} \quad .0666657 \$ \mathrm{H}$

$8016 \quad .0333343 \quad \$ O$

C

mpn1

82208

$1001.50 \mathrm{~m}$

8016

C

c Air $0.001205 \mathrm{~g} / \mathrm{cc}$

m2 $6012 \quad-0.000124 \quad \$ C$

$7014-0.755268 \$ \mathrm{~N}$

$8016 \quad-0.231781 \$ \mathrm{O}$

$18000-0.012827 \$ \mathrm{Ar}$

C

mpn2 6012

8016

8016

8016

c Water Calculated Density of $1 \mathrm{~g} / \mathrm{cc}$

m3 $1001 \quad$.666657 \$ H

$8016 \quad$ 333343 \$O

mt3 lwtr.01t

mpn3

1002

8016

C

C Fe Represnting a cask containing a criticallity accident

c Calculated Density $-7.874 \mathrm{~g} / \mathrm{cc}$

m4 $26056 \quad 1 \quad \$ \mathrm{Fe}$

C

mpn4

26056

C

phys:p 3j 1

C

MODE N P 
c Criticality Control Cards

c kcode 20001.050250

c ksrc 000

sdef pos 000 ERG=D1 RAD=D2

sp1 -3 0.9662 .842

$\mathrm{c}$

si2 019.539

C

c This is an Average Energy Deposited over a cell Tally

F6:N 3

SD6 7.28905E+02

C

F16:P 3

SD16 7.28905E+02

Calibration Analyses of the low field (80mrem/hr) NCD for building 325

c Detectors are calibrated in a neutron tank using a Cf-252 source. This source

c was born on 9-27-95 with an initial activity of .688Ci. Decay correct it is c .0208Ci as of $1-19-2008$.

c Modeled by Bryce Greenfield 1-19-2008

C

c Cell cards

c 2

3

3
4
5

$-0.001205 \quad(-28910)$

$-.93 \quad(-65) \quad$ imp:n=1

$-7.84 \quad(-324) \quad$ imp:n=1

1.7487e-5 (-5) imp: $\mathrm{n}=1$

-3.35 (-7 34$) \quad$ imp:n=1

$-0.001205 \quad$ (7 -99965 11) imp: $n=1$

$-0.001205 \quad(-4) \quad$ imp:n=1

$-7.84 \quad(-8) \quad$ imp:n=1

$-.93 \quad(-98) \quad$ imp:n=1

$-7.84 \quad(-10) \quad$ imp:n=1

$\begin{array}{llc}-.84 & (-12) & \text { imp: } n=1\end{array}$

$-1.17 \quad(-11) \quad$ imp:n=1

$-.93 \quad(-13) \quad$ imp: $\mathrm{n}=1$

-.93 (-14) imp:n=1

$\begin{array}{lll}-.93 & (-14) & \text { imp:n=1 } \\ -.93 & (-15) & \text { imp } n=1\end{array}$

c $17 \quad 3 \quad-.93 \quad(-16) \quad$ imp:n=1

c

9990

Blank line Follows-

c Surface cards

$\mathrm{C}$

2 RCC $000 \quad 0 \quad 00 \quad 998 \quad 15.24 \quad$ \$ Counting well 10m deep

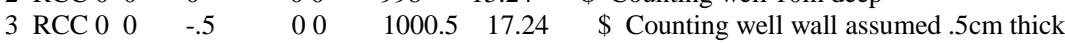

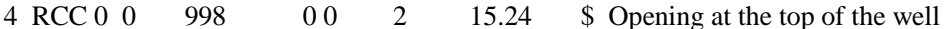

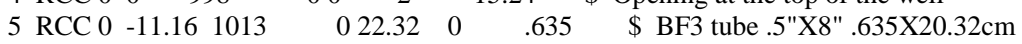

6 RCC 0 -10.15 $1013 \quad 020.3200 \quad 5.715 \quad$ \$ Polyethylene Moderating collar around BF3 tube 2.25"X8" 5.715X20.32cm

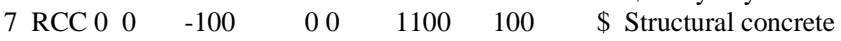

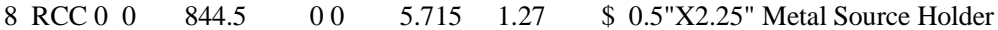

9 RCC $00 \quad 824.18 \quad 00 \quad 26.035 \quad 10.16 \quad \$ 4 " X 2.75 "$ Poly Colimator

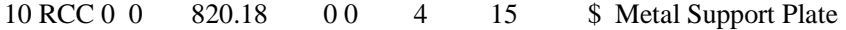

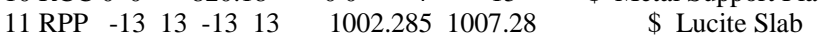

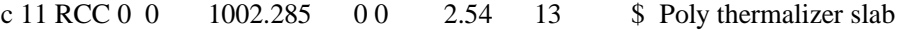

c 12 RPP -20 $20 \quad-20 \quad 20 \quad 1020 \quad 1026 \quad$ \$ Reflector Box top 


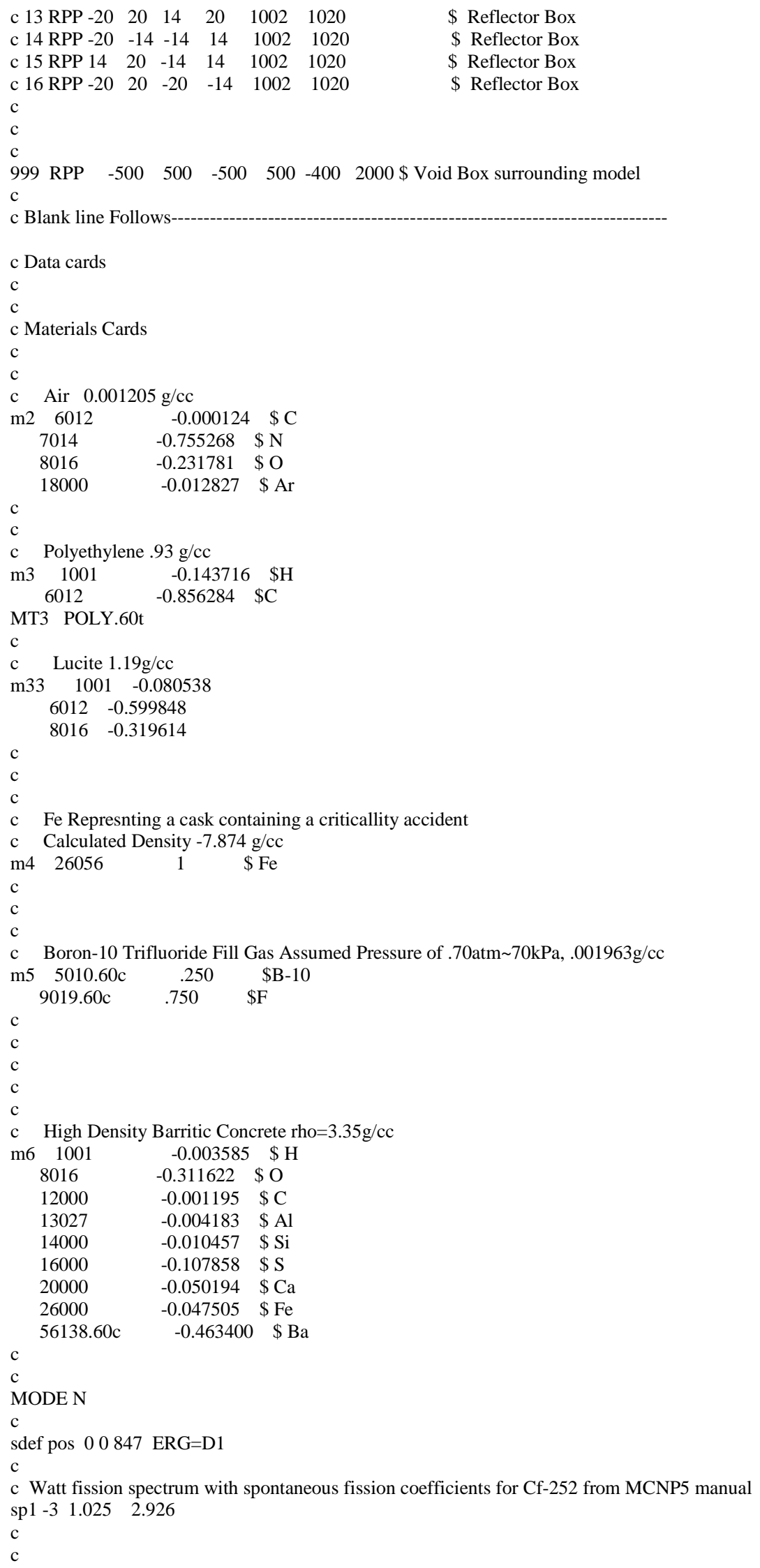


nps 5000000

C

c This is a current tally over the surface of the poly collar

c F2:N (6.1)

c 6.26 .3 )

c $.4 \mathrm{eV}$ is the Cd cutoff

c $4 \mathrm{eV}$ is a rough thermal cutoff where the Cd threshold becomes negligable

c

c E2 4E-6 20

C

c F12:N (5.1)

c E12 4E-6 20

c 5.25 .3$)$

c Surface area of BF3 tube

c SD2 162.145 .0675 .067

C

c Surface area of the Poly collar 934.875

c SD2 935

c Cell Flux Tally over poly collar

c 729.7102 .6102 .6

$\mathrm{F} 4: \mathrm{N} 3$

SD4 2059.26

E4 1.00E-07 1.00E-06 1.00E-05 1.00E-04 1.00E-03 1.00E-02

1.00E-01 5.00E-01 1.00E+00 2.00E+00 3.00E+00 4.00E+00

$5.00 \mathrm{E}+006.00 \mathrm{E}+00$

c Cell Flux Tally over poly collar

F14:N 3

SD14 2059.26

E14 4E-6 20

F1:N 11.5

C1 01

E1 4E-6 20 


\section{References}

ANSI/ANS-6.1.1-1991, "Neutron and Gamma-Ray Flux-to-Dose Rate Factors”, ANSI/ANS-6.1.1-1991 (N666), American Nuclear Society, LaGrange Park, Illinois (1991).

ANSI/ANS-8.1-1998, "Nuclear Criticality Safety in Operations with Fissionable Material Outside Reactors,” American Nuclear Society, LaGrange Park, Illinois (1998)

ANSI/ANS-8.3-1997, “Criticality Accident Alarm System,” American Nuclear Society, LaGrange Park, Illinois (1997).

ANSI/ANS-8.10-1983, “American National Standard Criteria for Nuclear Criticality Safety Controls in Operations with Shielding and Confinement”, American Nuclear Society, ANSI/ANS-8.10-1983, LaGrange park, Illinois (1983).

ANSI/ANS-8.15-1981, "Nuclear Criticality Control of Special Actinide Elements,” American Nuclear Society, La Grange Park, Illinois (1981).

ANSI/ANS-8.19-2005, “Administrative Practices for Nuclear Criticality Safety,” American Nuclear Society, LaGrange Park, Illinois (2005).

G. E. Hansen and W. H. Roach, "Six and Sixteen Group Cross Sections for Fast and Intermediate Critical Assemblies,” Los Alamos Scientific Laboratory report LAMS-2543, (December 6, 1961).

C.M. Hopper, and B.L. Broadhead, “An Updated Nuclear Criticality Slide Rule,” NUREG/CR 6504 vol. 2, April 1998.

Kim, Soon Sam, “NIST Sphere Experiments for U-235, Pu-239, U-238 and Np-237 Fission Rates” International Criticality Safety Benchmark Evaluation Program, 1995, Gaithersburg, Maryland. 
T. P. McLaughlin, S.P. Monahan, N.L. Pruvost, V.V. Frolov, B.G. Ryazanov, and V.I. Sviridov, "A Review of Criticality Accidents” Los Alamos National Laboratory, Los Alamos, New Mexico, LA-13638, May, 2000

Nuclear Energy Agency, “International handbook of Evaluated Criticality Safety Benchmark Experiments,” NEA/NSC Doc (95)03, September 2008

Reppond, E.P., "Criticality Safety Analysis Resource Book, Part Two: Atom Densities and Dimensional Parameters," Battelle Pacific Northwest Laboratories, 1977.

United States Department of Energy, “Facility Safety.” Order 420.1B, Approved 12-222005

X-5 Monte Carlo Team, MCNP5: A General Monte Carlo N-Particle Transport Code, Version 5, April 24, 2003 